\title{
Predicting Sea-Level Rise Vulnerability of Terrestrial Habitat and Wildlife of the Northwestern Hawailan Islands
}

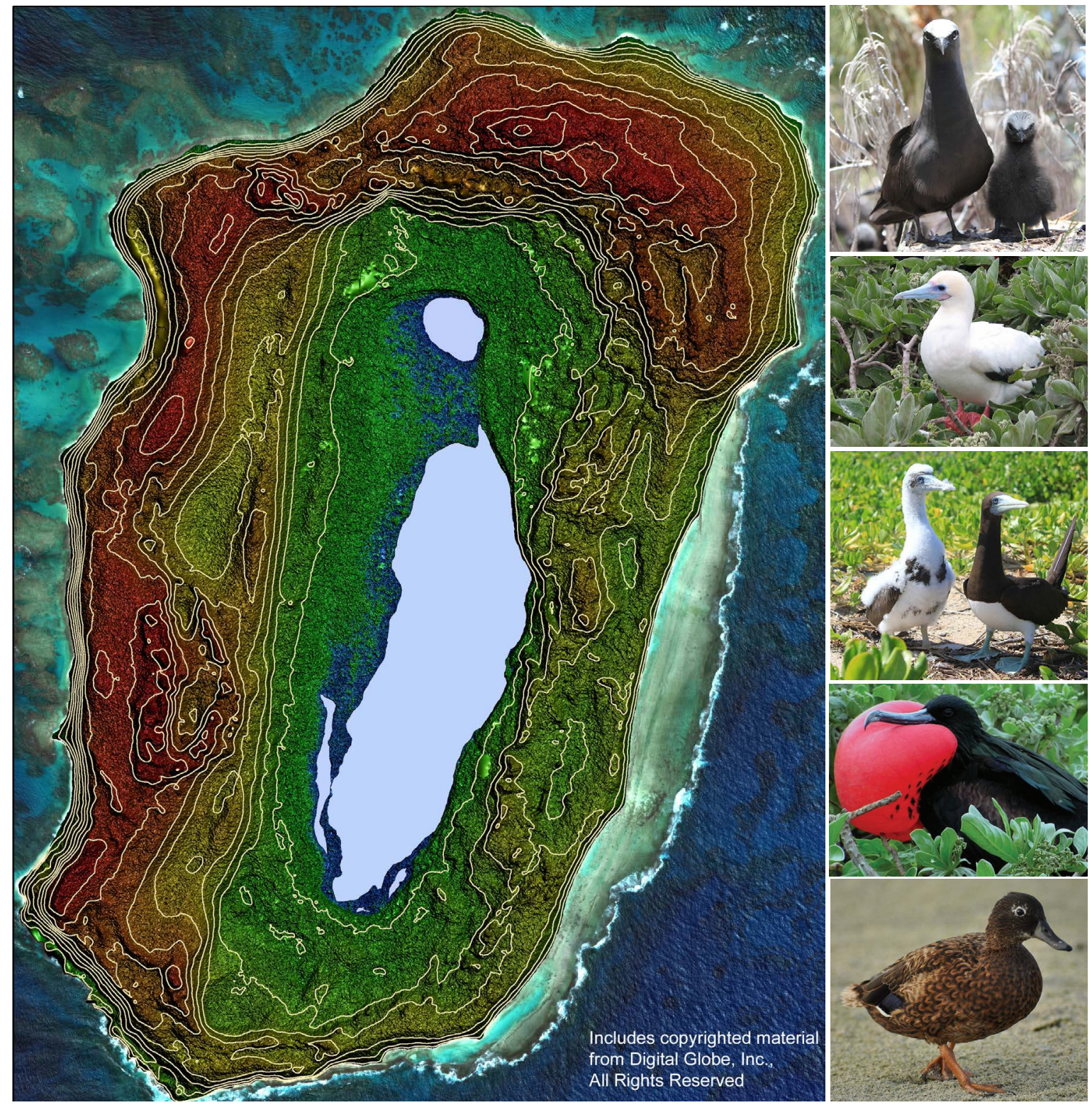

Open-File Report 2012-1182

U.S. Department of the Interior U.S. Geological Survey 
COVER

Photographs:

Black Noddy, Anous minutus marcusi, and chick (by T. Speetjens, U.S. Fish and Wildlife Service); Red-footed Booby, Sula sula rubripes (by C. Cornett, U.S. Fish and Wildlife Service);

Brown Booby, Sula leucogaster plotus, and chick (by T. Speetjens, U.S. Fish and Wildlife Service); Great Frigatebird, Fregata minor palmerstoni (by W. Taylor, U.S. Fish and Wildlife Service); and Laysan Teal, Anas laysanensis (by A. Boyd, U.S. Fish and Wildlife Service).

Map: Topographic image of Laysan Island (PhotoStat copyrighted image, used with permission). 


\section{Predicting Sea-Level Rise Vulnerability of Terrestrial Habitat and Wildlife of the Northwestern Hawaiian Islands}

Edited by Michelle H. Reynolds, Paul Berkowitz, Karen N. Courtot, and Crystal M. Krause

Open-File Report 2012-1182

U.S. Department of the Interior

U.S. Geological Survey 


\section{U.S. Department of the Interior KEN SALAZAR, Secretary \\ U.S. Geological Survey Marcia K. McNutt, Director}

\section{U.S. Geological Survey, Reston, Virginia: 2012}

This report and any updates to it are available online at: http://pubs.usgs.gov/of/2012/1182/

For more information on the USGS - the Federal source for science about the Earth, its natural and living resources, natural hazards, and the environment, visit http://www.usgs.gov or call 1-888-ASK-USGS

For an overview of USGS information products, including maps, imagery, and publications, visit http://www.usgs.gov/pubprod

To order this and other USGS information products, visit http://store.usgs.gov

Any use of trade, product, or firm names is for descriptive purposes only and does not imply endorsement by the U.S. Government.

Although this report is in the public domain, permission must be secured from the individual copyright owners to reproduce any copyrighted materials contained within this report.

Suggested citation:

Reynolds, M.H., Berkowitz, P., Courtot, K.N., and Krause, C.M., eds., 2012, Predicting sea-level rise vulnerability of terrestrial habitat and wildlife of the Northwestern Hawaiian Islands: U.S. Geological Survey Open-File Report 2012-1182, 139 p. 


\section{Contents}

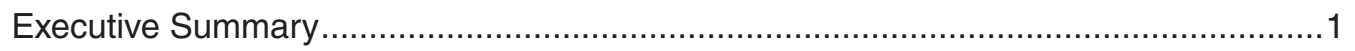

Chapter 1-Climate Change Vulnerability Assessment of the Low-Lying Northwestern Hawaiian Islands

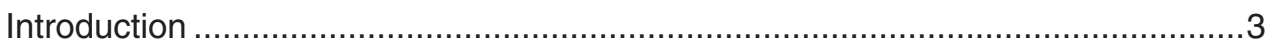

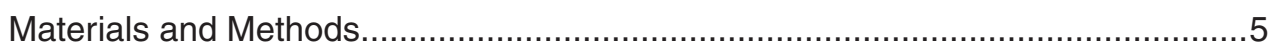

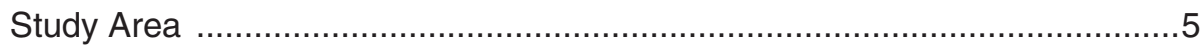

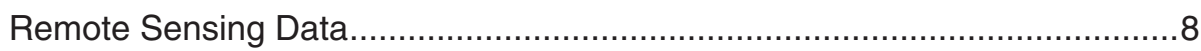

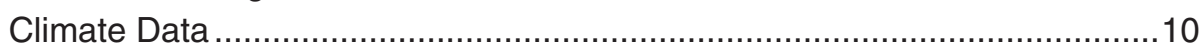

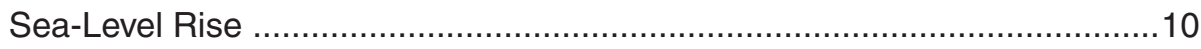

Land Cover Classification .......................................................................

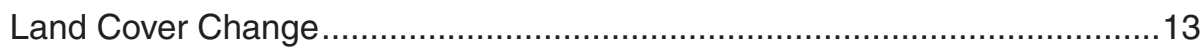

Avifauna Nesting Habitat Classification ....................................................13

Uncertainty and Model Assumptions ......................................................13

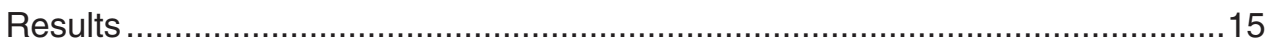

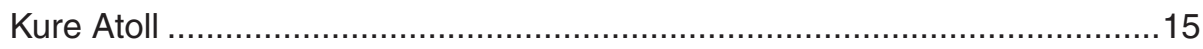

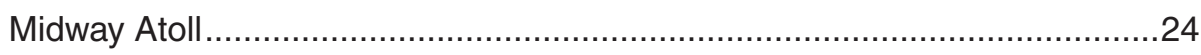

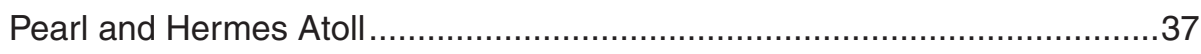

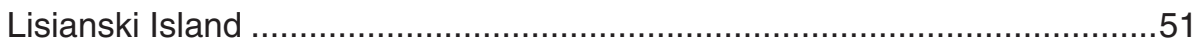

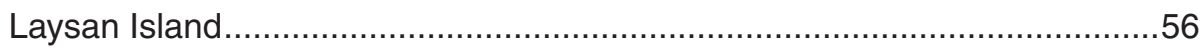

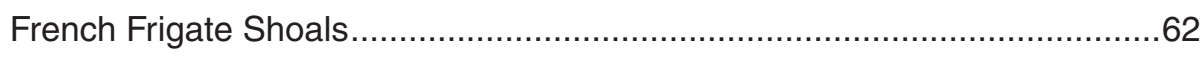

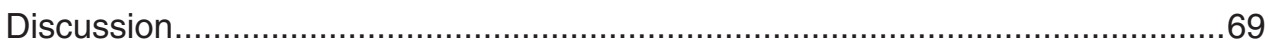

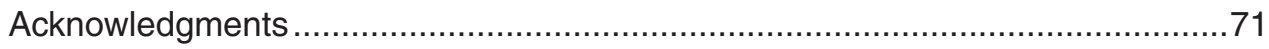

Chapter 2-Sea-Level Rise and Wave-Driven Inundation Models for Laysan Island

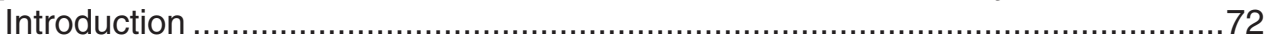

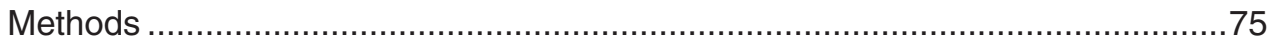

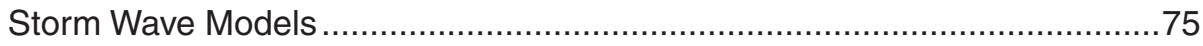

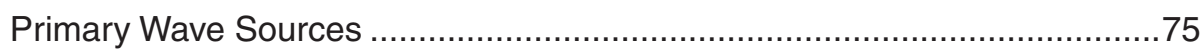

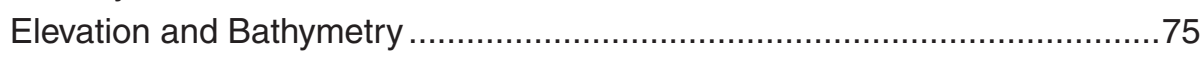

Modeled Water Surfaces and Wave Run-up ............................................79

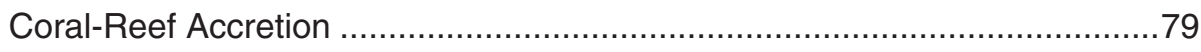

Estimating Mean Sea Level .............................................................. 82

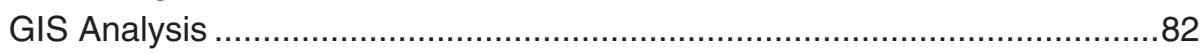

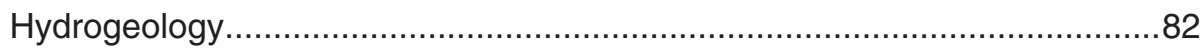

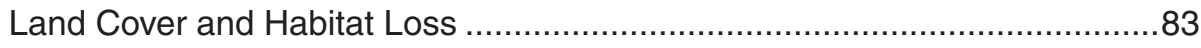

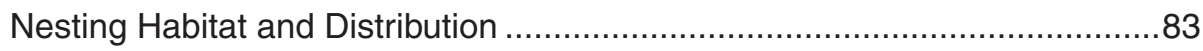

Nesting Phenology and Temporal Overlap with Storm Season ....................84

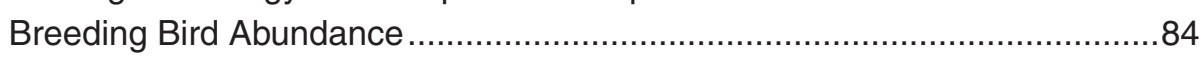

Wildlife Population Dynamics in Response to SLR .....................................85

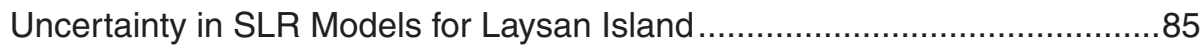

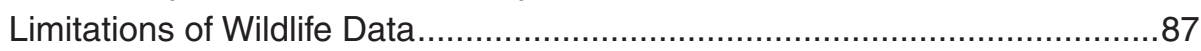




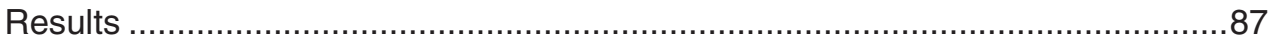

Sea-Level Rise and Wave-Driven Water Levels..........................................87

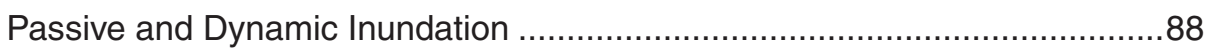

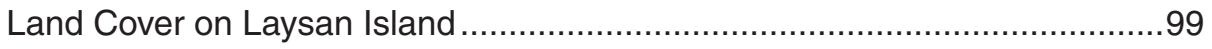

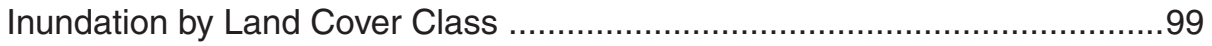

Impacts of SLR on Nesting Habitat ....................................................... 101

Vulnerability of Breeding Birds to Storm Conditions ...................................120

Population Dynamics, Carrying Capacity, and Population Vulnerability........122

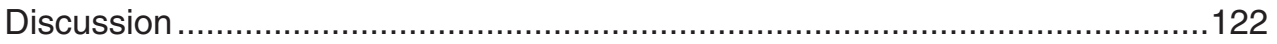

Systematic Underestimation of Vulnerability from Passive Models .............122

Additional Increments of SLR ............................................................ 122

Chronological Implications of Dynamic Inundation Models ........................122

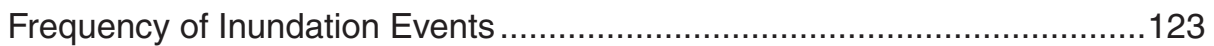

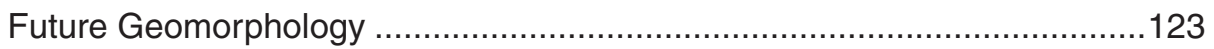

Vulnerability to Outlier Storm Events .....................................................123

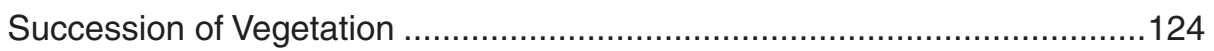

Thresholds of Vulnerability for Nesting Habitat ..........................................124

Implications of SLR for Laysan's Wildlife Populations...................................124

Future Research Directions................................................................. 125

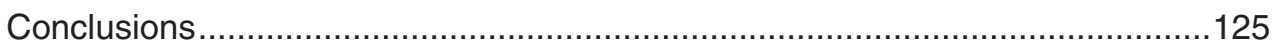

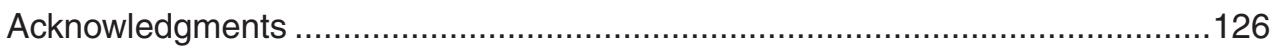

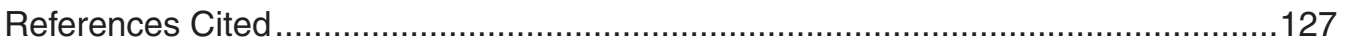

Appendix 1-USGS lidar data collection specifications ....................................136

Appendix 2-General circulation model data downscaled for analysis

of predicted temperature and precipitation 137

Appendix 3-Nesting descriptions of land birds and seabirds .........................137

\section{Figures}

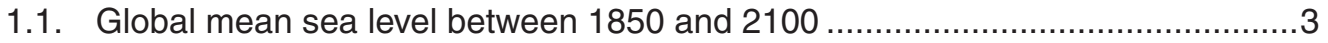

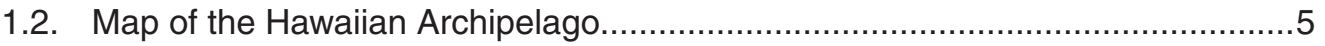

1.3. Schematic showing the lidar data collection process .......................................9

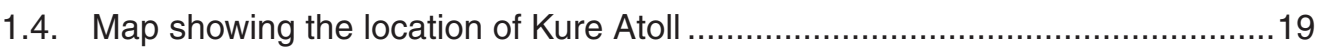

1.5. Green Island, Kure Atoll, land cover classification map .................................20

1.6. Topographic map of Green Island, Kure Atoll..............................................21

1.7. Green Island, Kure Atoll, passive inundation scenario maps ...........................22

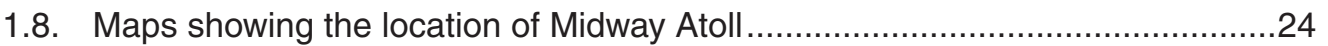

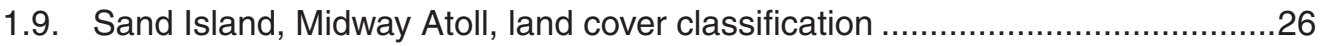

1.10. Spit Island, Midway Atoll, land cover classification map..................................27

1.11. Eastern Island, Midway Atoll, land cover classification map ............................28

1.12. Topographic map of Sand Island, Midway Atoll.............................................29

1.13. Sand Island, Midway Atoll, passive inundation scenario maps .........................30

1.14. Topographic map of Spit Island, Midway Atoll ............................................... 32

1.15. Spit Island, Midway Atoll, passive inundation scenario maps ..........................33

1.16. Topographic map of Eastern Island, Midway Atoll..........................................35

1.17. Eastern Island, Midway Atoll, passive inundation scenario maps ......................36 
1.18. Maps showing the location of Pearl and Hermes Atoll ...................................37

1.19. North Island, Pearl and Hermes Atoll, land cover classification map .................38

1.20. Southeast Island, Pearl and Hermes Atoll, land cover classification map..........39

1.21. Seal-Kittery Island, Pearl and Hermes Atoll, land cover classification map ......40

1.22. Topographic map of North Island, Pearl and Hermes Atoll ..............................43

1.23. Topographic map of Little North Island, Pearl and Hermes Atoll.......................44

1.24. Topographic map of Southeast Island, Pearl and Hermes Atoll ........................45

1.25. Topographic map of Grass Island, Pearl and Hermes Atoll..............................46

1.26. Topographic map of Seal-Kittery Island, Pearl and Hermes Atoll......................47

1.27. Pearl and Hermes Atoll passive inundation scenario maps .............................48

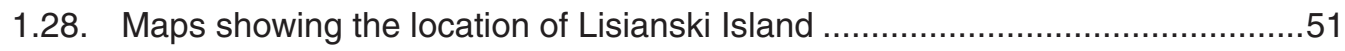

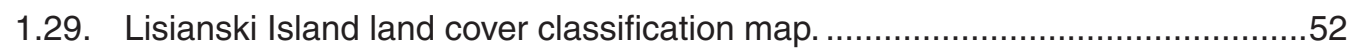

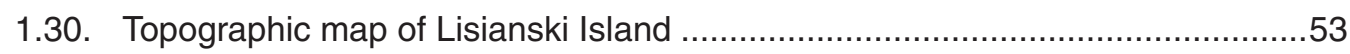

1.31. Lisianski Island passive inundation scenario maps ......................................54

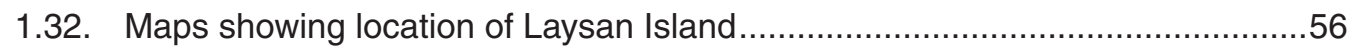

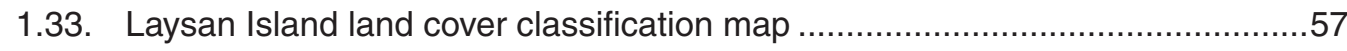

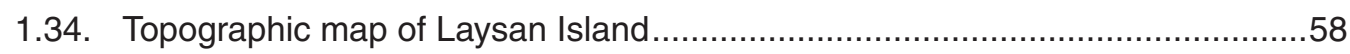

1.35. Laysan Island passive inundation scenario maps .........................................59

1.36. Laysan Island passive inundation scenario maps for five sea levels..................60

1.37. Topographic map of Tern Island, French Frigate Shoals .................................63

1.38. Topographic map of Trig Island, French Frigate Shoals..................................63

1.39. Topographic map of East Island, French Frigate Shoals ................................64

1.40. Topographic map of Gin Island, French Frigate Shoals...................................65

1.41. Topographic map of Little Gin Island, French Frigate Shoals ...........................66

1.42. Topographic map of Shark, Round and Disappearing Islands

of French Frigate Shoals....................................................................67

1.43. French Frigate Shoals passive inundation scenario maps ..............................68

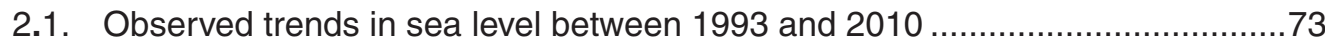

2.2. Schematic diagram showing the variation in waves and resulting wave-driven run-up at two sea levels ...........................................74

2.3. Schematic diagram comparing (1) GIS-based passive inundation models with (2) dynamic wave-driven inundation models ...........76

2.4. Time series showing monthly variations in Pacific Ocean wave heights, wave periods, and wind speeds ........................................................77

2.5. Compass plot of variation in wave height in meters ......................................78

2.6. Compass plot of variation in wave period in seconds ...................................78

2.7. Compass plot of variation in wind speed in meters per second ........................79

2.8. Seamless 100 -meter resolution bathymetric grid of Laysan Island...................80

2.9. Seamless 20 -meter resolution bathymetric and

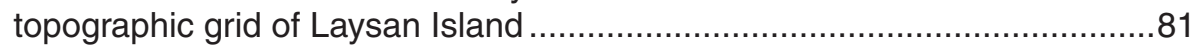

2.10. Conceptual model describing how global climate change may influence the environment and avifauna of Laysan Island .............................86

2.11. Map of Laysan Island depicting modeled wave-driven water levels ...................88

2.12. Overlay of passive inundation zones for five sea-level rise scenarios at Laysan Island. .90

2.13. Overlay of wave-driven inundation for five sea-level rise scenarios at Laysan Island. 
2.14. Chart of inundation extent in hectares at Laysan Island

for five sea-level rise scenarios

2.15. Map depicting potential inundation extent at Laysan Island under a sea-level rise scenario.

2.16. Map depicting potential inundation extent at Laysan Island under a sea-level rise scenario.

2.17. The inundation pattern documented by U.S. Fish and Wildlife

Service field staff on Laysan Island

2.18. Overlay of wave-driven inundation extents on Laysan Island

2.19. Overlay of passive inundation zones on Laysan Island for five sea-level rise scenarios

2.20. Overlay of wave-driven inundation including potential inundation from rising groundwater on Laysan Island for five sea-level rise scenarios ......................98

2.21. Land cover classes for Laysan Island ...................................................100

2.22. Overlay of Black-footed Albatross nesting habitat at Laysan Island.................105

2.23. Overlay of Laysan Albatross nesting habitat at Laysan Island .......................106

2.24. Overlay of Masked Booby and Brown Booby nests mapped on Laysan Island 108

2.25. Overlay of the shrub-nesting areas for Red-footed Booby and Great Frigatebird at Laysan Island

2.26. Overlay of Sooty Tern colonies at Laysan Island.

2.27. Overlay of Laysan Teal and Laysan Finch nesting habitat at Laysan Island ...115

2.28. Breeding phenology of 20 birds at Laysan Island 117

2.29. Population abundance graphs for five species

\section{Tables}

1.1. Known breeding distribution of avifauna, endangered plants, and other wildlife in the Northwestern Hawaiian Islands.

1.2. Northwestern Hawaiian Islands airborne lidar data collection information..........9

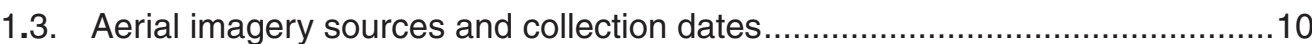

1.4. Climate change general circulation models ...................................................10

1.5. National Oceanic and Atmospheric Administration tide values ........................11

1.6. Land cover class definitions with plant species for each class.........................12

1.7. Land cover classes identified for the low-lying Northwestern Hawaiian Islands from satellite imagery. ................................................13

1.8. Known avifauna nesting habitat by land cover class ........................................14

1.9. Mean and maximum elevations of Northwestern Hawaiian Islands ..................16

1.10. Global breeding distribution for species of the Northwestern Hawaiian Islands ..............................................................17

1.11. Conservation status assessments and population counts of species breeding in the Northwestern Hawaiian Islands .18

1.12. Predicted average annual maximum temperature and total precipitation .........19

1.13. Total land area and percent change in area for each of six land cover types at Green Island, Kure Atoll

1.14. Total potential nesting habitat area and percent change in nesting habitat area for breeding avifauna at Green Island, Kure Atoll 
1.15. Total land area and percent change in area for each of ten land cover types at Sand Island, Midway Atoll .............................................................25

1.16. Total land area and percent change in area for each of six land cover types at Spit Island, Midway Atoll ..............................................................25

1.17. Total land area and percent change for each of five land cover types at Eastern Island, Midway Atoll .......................................................25

1.18. Total potential nesting habitat area and percent change in nesting habitat area for breeding avifauna at Sand Island, Midway Atoll

1.19. Total potential nesting habitat area and percent change in nesting habitat area for breeding avifauna at Spit Island, Midway Atoll......

1.20. Total potential nesting habitat area and percent change for breeding avifauna at Eastern Island, Midway Atoll,

1.21. Total land area and percent change for each of four land cover types at North Island, Pearl and Hermes Atoll.....

1.22. Total land area and percent change for each of two land cover types at Little North Island, Pearl and Hermes Atoll ....

1.23. Total land area and percent change for each of six land cover types at Southeast Island, Pearl and Hermes Atoll.

1.24. Total land area and percent change for each of three land cover types at Grass Island, Pearl and Hermes Atoll

1.25. Total land area and percent change for each of four land cover types at Seal-Kittery Island, Pearl and Hermes Atoll...

1.26. Total potential nesting habitat area and percent change for breeding avifauna at North Island, Pearl and Hermes Atoll

1.27. Total potential nesting habitat area and percent change for breeding avifauna at Little North Island, Pearl and Hermes Atoll

1.28. Total potential nesting habitat area and percent change for breeding avifauna at Southeast Island, Pearl and Hermes Atoll.....

1.29. Total potential nesting habitat area and percent change for breeding avifauna at Grass Island, Pearl and Hermes Atoll ..........................50

1.30. Total potential nesting habitat area and percent change for breeding avifauna at Seal-Kittery Island, Pearl and Hermes Atoll ...................50

1.31. Total land area and percent change for each of five land cover types at Lisianski Island

1.32. Total potential nesting habitat area and percent change for breeding avifauna at Lisianski Island

1.33. Total potential nesting habitat area and percent change for breeding avifauna at Laysan Island

2.1. Recent published estimates of sea-level rise (in meters) by the end of the 21st century.

2.2. Inundation extent for five sea-level scenarios and three modeling approaches

2.3. Area for each land cover class on Laysan Island, including percentage of total terrestrial area and percentage of total island area.

2.4. Inundated areas and percent change for Laysan Island, tallied by land cover class for five sea-level rise scenarios

2.5. Inundation of 2009-2010 nesting habitat at Laysan Island for Black-footed Albatross and Laysan Albatross. 
2.6. Inundation of 2009 nest sites at Laysan Island for Masked Booby and Brown Booby.

2.7. Inundated 2008 nesting habitat at Laysan Island for the Red-footed Booby and Great Frigatebird

2.8. Inundated colony areas at Laysan Island for Sooty Tern

2.9. Inundated nesting habitat at Laysan Island for Laysan

Teal and Laysan Finch

2.10. Life-history traits for 20 avian species on Laysan Island

2.11. Population parameter estimates for the Gompertz model

\section{Conversion Factors and Datum}

\begin{tabular}{lll}
\multicolumn{1}{l}{ SI to Inch/Pound } & \multicolumn{1}{c}{ Mo obtain } \\
\hline & Area & \\
\hline square meter $\left(\mathrm{m}^{2}\right)$ & 0.0002471 & acre \\
hectare $(\mathrm{ha})$ & 2.471 & acre \\
square kilometer $\left(\mathrm{km}^{2}\right)$ & 247.1 & acre \\
hectare (ha) & 0.003861 & square mile $\left(\mathrm{mi}^{2}\right)$ \\
square kilometer $\left(\mathrm{km}^{2}\right)$ & 0.3861 & square mile $\left(\mathrm{mi}^{2}\right)$ \\
\hline & Length & \\
\hline millimeter $(\mathrm{mm})$ & 0.03937 & inch (in) \\
centimeter $(\mathrm{cm})$ & 0.3937 & inch (in) \\
meter $(\mathrm{m})$ & 3.281 & foot $(\mathrm{ft})$ \\
kilometer $(\mathrm{km})$ & 0.6214 & mile (mi) \\
\hline & Rate & \\
\hline millimeter per year $(\mathrm{mm} / \mathrm{yr})$ & 0.03937 & inch per year (in/yr) \\
\hline
\end{tabular}

Temperature in degrees Celsius $\left({ }^{\circ} \mathrm{C}\right)$ may be converted to degrees Fahrenheit $\left({ }^{\circ} \mathrm{F}\right)$ as follows: ${ }^{\circ} \mathrm{F}=\left(1.8 \times{ }^{\circ} \mathrm{C}\right)+32$

Vertical coordinate information is referenced to local mean sea level.

Horizontal coordinate information is referenced to the North American Datum of 1983 (NAD 83).

Altitude, as used in this report, refers to distance above the vertical datum.

Universal Transverse Mercator (UTM), zones 1-3, is the coordinate system.

Elevation data were provided relative to mean sea level (MSL) in meters. 


\section{Abbreviations and Acronyms}

\begin{tabular}{|c|c|}
\hline AR4 & Fourth Assessment Report, Intergovernmental Panel on Climate Change \\
\hline $\mathrm{BCC}$ & Birds of Conservation Concern \\
\hline BFAL & Black-footed Albatross \\
\hline $\mathrm{BRBO}$ & Brown Booby \\
\hline Deltares & Dutch Institute for Delta Technology \\
\hline DEM & Digital elevation model \\
\hline DLNR & Hawai'i Department of Land and Natural Resources \\
\hline GCM & General circulation model \\
\hline GPS & Global Positioning System \\
\hline GRFR & Great Frigatebird \\
\hline GRS 80 & Geodetic Reference System 1980 \\
\hline HTDP & Horizontal Time Dependent Positioning \\
\hline HWM & High-water mark \\
\hline IMU & Inertial measurement unit \\
\hline IPCC & Intergovernmental Panel on Climate Change \\
\hline ITRF00 & International Terrestrial Reference Frame of 2000 \\
\hline IUCN & International Union for Conservation of Nature \\
\hline LAAL & Laysan Albatross \\
\hline LADU & Laysan Teal \\
\hline LAFI & Laysan Finch \\
\hline lidar & Light detection and ranging \\
\hline loran & Long-range aide to navigation \\
\hline MABO & Masked Booby \\
\hline MHW & Mean high water \\
\hline $\mathrm{MIC}$ & Mean incubation count \\
\hline MLLW & Mean lower low water \\
\hline MLW & Mean low water \\
\hline NGS & National Geodetic Survey \\
\hline NOAA & National Oceanic and Atmospheric Administration \\
\hline NWHI & Northwestern Hawaiian Islands \\
\hline NWR & National wildlife refuge \\
\hline OPUS & Online positioning user service \\
\hline PDOP & Positional dilution of precision \\
\hline PIBHMC & Pacific Island Benthic Habitat Mapping Center \\
\hline PMNM & Papahānaumokuākea Marine National Monument \\
\hline RFBO & Red-footed Booby \\
\hline RMSE & Root-mean-squared error \\
\hline RTK & Real-time kinematic \\
\hline SD & Standard deviation \\
\hline SLOSH & Sea, Lake, and Overland Surges from Hurricanes \\
\hline SLR & Sea-level rise \\
\hline SOTE & Sooty Tern \\
\hline TIN & Triangulated irregular network \\
\hline UNESCO & United Nations Educational Scientific and Cultural Organization \\
\hline USACE & U.S. Army Corps of Engineers \\
\hline USFWS & U.S. Fish and Wildlife Service \\
\hline USGS & U.S. Geological Survey \\
\hline WGS 84 & World Geodetic System 1984 \\
\hline
\end{tabular}


This page intentionally left blank. 


\title{
Predicting Sea-Level Rise Vulnerability of Terrestrial Habitat and Wildlife of the Northwestern Hawaiian Islands
}

\author{
Edited by Michelle H. Reynolds, Paul Berkowitz, Karen N. Courtot, and Crystal M. Krause
}

\section{Executive Summary}

If current climate change trends continue, rising sea levels may inundate low-lying islands across the globe, placing island biodiversity at risk. Recent models predict a rise of approximately one meter $(1 \mathrm{~m})$ in global sea level by 2100 , with larger increases possible in areas of the Pacific Ocean. Pacific Islands are unique ecosystems home to many endangered endemic plant and animal species. The Northwestern Hawaiian Islands (NWHI), which extend 1,930 kilometers $(\mathrm{km})$ beyond the main Hawaiian Islands, are a World Heritage Site and part of the Papahānaumokuākea Marine National Monument. These NWHI support the largest tropical seabird rookery in the world, providing breeding habitat for 21 species of seabirds, 4 endemic land bird species and essential foraging, breeding, or haul-out habitat for other resident and migratory wildlife. In recent years, concern has grown about the increasing vulnerability of the NWHI and their wildlife populations to changing climatic patterns, particularly the uncertainty associated with potential impacts from global sealevel rise (SLR) and storms.

In response to the need by managers to adapt future resource protection strategies to climate change variability and dynamic island ecosystems, we have synthesized and down scaled analyses for this important region. This report describes a 2-year study of a remote northwestern Pacific atoll ecosystem and identifies wildlife and habitat vulnerable to rising sea levels and changing climate conditions. A lack of high-resolution topographic data for low-lying islands of the NWHI had previously precluded an extensive quantitative model of the potential impacts of SLR on wildlife habitat. The first chapter (chapter 1) describes the vegetation and topography of 20 islands of Papahānaumokuākea Marine National Monument, the distribution and status of wildlife populations, and the predicted impacts for a range of SLR scenarios. Furthermore, this chapter explores the potential effects of SLR on wildlife breeding habitats for each island. The subsequent chapter (chapter 2) details a study of the Laysan Island ecosystem, describing a quantitative model that incorporates SLR, storm wave, and rising groundwater inundation.
Wildlife, storm, and oceanographic data allowed for an assessment of the phenological and spatial vulnerability of Laysan Island's breeding bird species to SLR and storms.

Using remote sensing and geospatial techniques, we estimated topography, classified vegetation, modeled SLR, and evaluated a range of climate change scenarios. On the basis of high-resolution airborne data collected during 2010-11 (root-mean-squared error $=0.05-0.18 \mathrm{~m}$ ), we estimated the maximum elevation of 20 individual islands extending from Kure Atoll to French Frigate Shoals (range: 1.8-39.7 m) and computed the mean elevation $(1.7 \mathrm{~m}$, standard deviation $1.1 \mathrm{~m})$ across all low-lying islands. We also analyzed general climate models to describe rainfall and temperature scenarios expected to influence adaptation of some plants and animals for this region. Outcomes for the NWHI predicted an increase in temperature of 1.8-2.6 degrees Celsius $\left({ }^{\circ} \mathrm{C}\right)$ and an annual decrease in precipitation of 24.7-76.3 millimeters $(\mathrm{mm})$ across the NWHI by 2100.

Our models of passive SLR (excluding wave-driven effects, erosion, and accretion) showed that approximately 4 percent of the total land area in the NWHI will be lost with scenarios of $+1.0 \mathrm{~m}$ of SLR and 26 percent will be lost with $+2.0 \mathrm{~m}$ of SLR. Some atolls are especially vulnerable to SLR. For example, at Pearl and Hermes Atoll our analysis indicated substantial habitat losses with 43 percent of the land area inundated at $+1.0 \mathrm{~m}$ SLR and 92 percent inundated at +2.0 $\mathrm{m}$ SLR. Across the NWHI, seven islands will be completely submerged with $+2.0 \mathrm{~m}$ SLR. The limited global ranges of some tropical nesting birds make them particularly vulnerable to climate change impacts in the NWHI. Climate change scenarios and potential SLR impacts presented here emphasize the need for early climate change adaptation and mitigation planning, especially for species with limited breeding distributions and/or ranges restricted primarily to the low-lying NWHI: Cyperus pennatiformis var. bryanii, Black-footed Albatross (Phoebastria nigripes), Laysan Albatross (P. ітmиtabilis), Bonin Petrel (Pterodroma hypoleuca), Gray-backed Tern (Onychoprion lunatus), Laysan Teal (Anas laysanensis), 
Laysan Finch (Telespiza cantans), and Hawaiian monk seal (Monachus schauinslandi). Furthermore, SLR scenarios that include the effects of wave dynamics and groundwater rise may indicate amplified vulnerability to climate change driven habitat loss on low-lying islands.

In chapter 2, we incorporated the combined effects of SLR, dynamic wave-driven inundation, and rising groundwater in a quantitative study specifically for the Laysan Island ecosystem. This is the first hydrodynamic model to simulate the combined impacts of SLR and wave-driven inundation in the NWHI. We developed a high-resolution digital elevation model (mean vertical accuracy of $0.32 \mathrm{~m}$ ) for the island. Then using recent satellite imagery, geospatial models, and historical oceanographic, storm, and biological data we estimated potential inundation extent, habitat loss, and wildlife population impacts for a range of potential SLR scenarios $(0.00$, $+0.50,+1.00,+1.50$, and $+2.00 \mathrm{~m}$ ) that may occur over the next century. Additionally, we estimated the carrying capacity of Laysan Island for five species based on the available population monitoring data and described how potential losses in nesting habitat could influence population dynamics for Black-footed Albatross, Laysan Albatross, Red-footed Booby (Sula sula), Laysan Teal, and Laysan Finch. For some other seabird populations (Masked Booby, S. dactylatra; Brown Booby, S. leucogaster; Great Frigatebird, Fregata minor; and Sooty Tern, Onychoprion fuscata), we used recent colony distribution data, land cover maps, and nesting behavior to estimate potential losses of nesting habitat from SLR and wave-driven inundation.

We observed far greater potential impacts of SLR to wildlife with the dynamic wave-driven modeling approach than with the passive modeling approach. Depending on SLR scenario and coastal orientation, during storms under $\mathrm{a}+2.00$ $\mathrm{m}$ SLR scenario, the wave-driven inundation model predicted three times more inundation than the passive model (17.2 percent of total terrestrial area versus 4.6 percent, respectively). Large-wave events generally added $1 \mathrm{~m}$ of water height to passive inundation surfaces, therefore our dynamic models (during storm events) forecasted comparable inundation extents earlier than passive models. Although wave-driven water levels were highest in the northwest quadrant of Laysan
Island, the greatest extent of inundation occurred in the southeast where coastal dunes less than $3 \mathrm{~m}$ above mean sea level provide little protection from wave-driven inundation.

When wave-driven inundation was included in the SLR model for Laysan Island greater nesting habitat loss and potential impacts on wildlife population dynamics were predicted. The consequences of habitat loss due to SLR may be worse for species with colonies in the wave-exposed coastal zones (for example, Black-footed Albatross) and for populations already near the island's carrying capacity (for example, Laysan Teal). Species whose peak incubation and chick-rearing periods coincide with seasonally high wave heights also will be increasingly vulnerable, especially those species nesting on the ground in areas vulnerable to inundation, such as Graybacked Tern and Black-footed Albatross. Other species that have space for population growth, or are not restricted to a narrow range of habitat types on Laysan (for instance, Sooty Terns), may be less sensitive to habitat loss from SLR over the next century.

Our assessments of inundation risk, habitat loss, and wildlife species vulnerability synthesize current knowledge about individual islands and contribute to a broader understanding of the impacts of inundation from SLR and storm-induced waves. Yet, most NWHI and their bird populations lack monitoring data to evaluate adaptations to and impacts of climate change. Exceptions include some data sets from long-term monitoring of wildlife populations, tides, or weather at French Frigate Shoals, Laysan Island, and Midway Atoll. These data sets are potentially valuable baselines, which could be informative for adaptive learning (integrating management and science) to predict, adapt, and mitigate the effects of climate change on NWHI wildlife in the future. This study provides the first quantitative vulnerability assessment for all of the low-lying NWHI, and results identify biological communities, locales, and resident endangered species of Papahānaumokuākea Marine National Monument expected to be at risk from SLR. This report is also intended as a reference for managers and conservation planners, a tool to identify and potentially reduce uncertainty, and a starting place for developing climate change monitoring priorities and future scientific studies. 


\title{
Chapter 1
}

\section{Climate Change Vulnerability Assessment of the Low-Lying Northwestern Hawaiian Islands}

\author{
By Crystal M. Krause ${ }^{1}$, Karen N. Courtot ${ }^{1}$, Paul Berkowitz ${ }^{2}$, Jamie Carter ${ }^{3}$, and Michelle H. Reynolds ${ }^{1}$
}

\section{Introduction}

Rapid climate change, namely the global trend of atmospheric warming, is an important factor expected to contribute to numerous extinctions of plant and animal species across the globe. Although recent studies describe the natural adaptive capacity of many species including phenological changes in reproduction, seasonal wildlife migration, and shifting range boundaries (Parmesan, 2006; Lawler and others, 2009), many ecosystems have been identified as vulnerable to negative impacts of climate change. Arctic tundra, wetland coastal communities, and small island ecosystems are recognized as particularly vulnerable and may require intensive mitigation to prevent species losses due to climate change and other stressors (Intergovernmental Panel on Climate Change (IPCC), 2007).

\footnotetext{
${ }^{1}$ U.S. Geological Survey, Pacific Island Ecosystems Research Center, Kīlauea Field Station, Hawai'i National Park, HI 96718.

${ }^{2}$ Hawai'i Cooperative Studies Unit, University of Hawai'i at Hilo, Hilo, HI 96720.

${ }^{3}$ National Oceanic and Atmospheric Administration, Pacific Services Center, Honolulu, HI 96813.
}

Sea level represents a very sensitive index of global climate change (Cazenave and Llovel, 2010). Warming of the earth's atmosphere affects sea level by (1) raising oceanic temperatures and causing thermal expansion of ocean waters, (2) increasing freshwater inputs from glacial melting, and (3) altering the mass balance of major ice sheets, resulting in increased global water volumes. Recent estimates of sealevel rise (SLR) that incorporate Greenland and Antarctic ice melt predict that SLR could exceed 1.0 meter $(\mathrm{m})$ by 2100 (Rahmstorf, 2007; Vermeer and Rahmstorf, 2009), with more rapid increases if major collapses of the polar ice sheets occur (Overpeck and others, 2006). From 1962 through 1990, global sea levels have risen at a mean rate of $1.5 \pm 0.5$ millimeter per year (mm/yr). Since 1990, the trend has increased to 3.2 $\pm 0.4 \mathrm{~mm} / \mathrm{yr}$ (Merrifield and others, 2009); further acceleration is possible (Church and White, 2006; Jevrejeva and others, 2008; Rahmstorf, 2010). Recent satellite altimetry has shown that in the western Pacific Ocean, including areas in the vicinity of the Northwestern Hawaiian Islands (NWHI), much higher rates of SLR (5-10 mm/yr) have occurred from 1992
Figure 1.1. Global mean sea level between 1850 and 2100, modified from Cazenave and Llovel (2010). The thick black line indicates historical sea levels and the red line shows sea levels from observed tide gauge data (Church and White, 2011). The purple, blue, and orange areas show sea-level rise projections and the increasing uncertainty associated with projections after 2050, based on three different climate models from the Intergovernmental Panel on Climate Change (IPCC, 2007): purple without scaled-up ice sheet discharge, blue with scaled-up ice sheet discharge, and orange after Rahmstorf's (2007) semiempirical approach to projecting future sea-level rise.

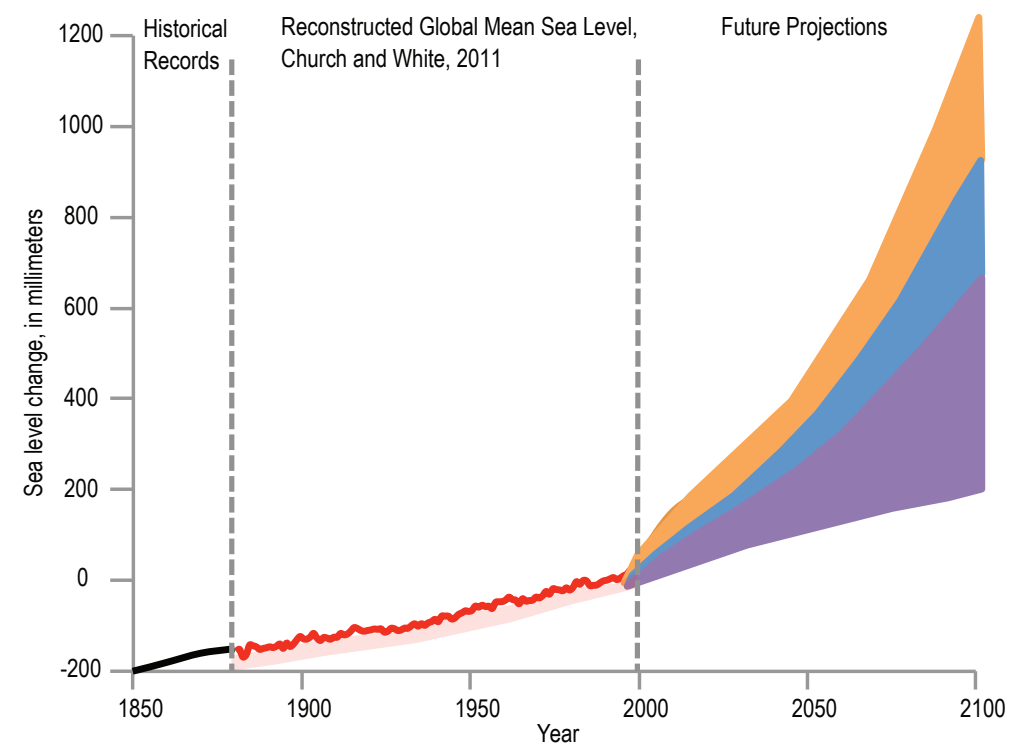


to 2011(Leuliette, 2012). Historical trends in sea level for the past two centuries as well as three different projections of sea level for this century (2000-2100) are shown in fig. 1.1 (IPCC, 2007; Rahmstorf, 2007; Cazenave and Llovel, 2010).

The Intergovernmental Panel on Climate Change (IPCC) Fourth Assessment Report (AR4; 2007) identified several potential impacts of SLR, including reductions in island size, loss of freshwater resources, damage to coral reefs, declines in fisheries, deterioration of marine resources, and erosion of shorelines. Additionally, increases in sea surface temperatures may increase the intensity of storms, amplifying the effects of SLR with storm-generated impacts and flooding (IPCC, 2007; Mousavi and others, 2011). The combination of SLR, storm surge, wave run-up (that is, the maximum vertical extent of waves on a beach), and coastal erosion poses risks to low-lying islands in the Pacific, as well as to the species that inhabit them.

Few studies have examined the consequences of SLR on the biodiversity of low-lying islands, with most studies analyzing the effects of SLR using passive inundation scenarios (also known as "bathtub" models; Baker and others, 2006; Zhang and others, 2011). A recent global assessment of the effects of SLR on biodiversity predicted inundation of 0.7 percent of the world's terrestrial area (1.1 million square kilometers, $\mathrm{km}^{2}$ ) under a $+1.0 \mathrm{~m}$ SLR scenario (Menon and others, 2010). However, the Menon study relied on relatively coarse biological and topographic data and produced first-order estimates of passive inundation for all terrestrial ecoregions globally.

In the central Pacific, rapid climate change threatens Hawai'i's wildlife in numerous ways, including increased transmission of avian malaria for Hawaiian native forest birds (Benning and others, 2002; LaPointe and others, 2010), threats to coral reefs such as bleaching and ocean acidification (Jokiel and Brown, 2004), and SLR-induced habitat loss on small islands (Baker and others, 2006). Worst-case scenarios of SLR predict inundation of many low-lying islands and high extinction risk for many island endemics (that is, species confined to and indigenous to the region). Climate change projections for the main Hawaiian Islands include a 5-10-percent reduction in rainfall during the wet season (November-April) and a 5-percent increase in rainfall during the dry season (May-October), primarily due to changes in mid-latitude cyclone frequency and trade wind patterns, respectively (Timm and Diaz, 2009). Daily temperature ranges in the main Hawaiian Islands are decreasing while average ambient temperatures at sea level are projected to increase by 2100 (IPCC, 2007). Changes in precipitation and temperature will likely affect vegetation distributions and composition (Juvik and others, 2011) and potentially increase ecosystem vulnerability to invasion by non-native species (Masters and Norgrove, 2010).

The Northwestern Hawaiian Islands (NWHI) extend 1,930 kilometers $(\mathrm{km})$ beyond the main Hawaiian Islands and consist of Kure Atoll (Mokupāpapa), Midway Atoll (Pihemanu), Pearl and Hermes Atoll (Holoikauaua), Lisianski Island (Papa'āpoho), Laysan Island (Kaūo), Maro Reef (Ko'anako'a, Nalukākala; largely submerged), Gardner Pinnacles (Pūhāhonu), French Frigate Shoals (Kānemiloha'i), Mokumanamana (Necker Island), and Nihoa. The geographic isolation of the NWHI has led to the development of an extraordinary range of intact marine and terrestrial ecosystems that include a high degree of endemism (Conant and others, 1984; Friedlander and others, 2009). The NWHI are home to many endangered endemic plant and animal species and support the largest tropical seabird rookery in the world (United Nations Educational Scientific and Cultural Organization (UNESCO), 2010). For a recent assessment of the diverse marine ecosystems of the NWHI, see Friedlander and others (2009). The NWHI provide habitat for over 14 million federally protected tropical seabirds (Fefer and others, 1984), 4 resident land bird species, and 90 percent of Hawai'i's subpopulation of green turtles (Chelonia mydas); this area is also designated critical habitat for the Hawaiian monk seal (Monachus schauinslandi) and five species of endangered plants (National Oceanic and Atmospheric Administration (NOAA), 1986; 1988; U.S. Fish and Wildlife Service (USFWS), 2003; 2005). The NWHI are also an important migration stopover or overwintering habitat for at least 29 species of migratory birds of the Pacific flyway (Pyle and Pyle, 2009).

The terrestrial ecosystems of the remote NWHI remained largely intact until the late 1800 s, when guano mining, feather and egg harvesting, and mammalian introductions contributed to population declines and the extinction of numerous endemic species (Bryan, 1911; Farrell, 1928; Bailey, 1956). All of the NWHI except for Midway Atoll, which was under U.S. military jurisdiction at the time, were protected by the 1909 Bird Reserve designation (Executive Order 1019). Additional conservation designations occurred later, including the creation of the Hawaiian Islands National Wildlife Refuge in 1940 (Presidential Proclamation 2416), Midway Atoll National Wildlife Refuge in 1996 (Executive Order 13022), and Papahānaumokuākea Marine National Monument (PMNM) in 2006 (Presidential Proclamation 8112). Papahānaumokuākea Marine National Monument was designated a World Heritage Site in 2010 (UNESCO, 2010). The NWHI conservation area encompasses $362,061 \mathrm{~km}^{2}$ of oceanic and terrestrial habitat (Friedlander and others, 2009).

In order to describe terrestrial habitat vulnerabilities to SLR across this remote archipelago, we collected new topographic data for Kure Atoll, Midway Atoll, Pearl and Hermes Atoll, Lisianski Island, Laysan Island (see chap. 2), and French Frigate Shoals (see Hatfield and others, 2012), as well as compiled species distribution and habitat information for breeding birds, endangered plants, and other wildlife. We then modeled the potential impacts of climate change and SLR in order to describe possible effects of inundation on wildlife habitat of the NWHI with an emphasis on breeding birds. 


\section{Materials and Methods}

\section{Study Area}

Papahānaumokuākea Marine National Monument, including small islands, atolls, submerged banks, and reefs, is the largest conservation area in the United States (Papahānaumokuākea Marine National Monument, 2008; fig.1.2). The land area included in this study consists of four multi-island coral atolls (Kure, Midway, Pearl and Hermes, and French Frigate Shoals), two carbonate islands (Lisianski and Laysan), and two basalt islands (Mokumanamana and Nihoa). The diverse ecosystems of the NWHI support 21 breeding seabirds (table 1.1) and 17 terrestially-breeding species of animals and plants listed as endangered, threatened, or species of concern by the USFWS, State of Hawai' $i$, or International Union for Conservation of Nature including: Hawaiian monk seal or 'ilio-holo-i-ka-uaua, green turtle or honu, Laysan Teal (Anas laysanensis), Laysan Finch or 'Ainohu kauo (Telespiza cantans), Nihoa Finch (T. ultima), Nihoa Millerbird (Acrocephalus familiaris subsp. kingi), Short-tailed Albatross (Phoebastria albatrus), Black-footed Albatross or Ka'upu (P. nigripes), Laysan Albatross or Mōlī (P. immutabilis), Christmas Shearwater (Puffinus nativitatis), Tristram's Storm-petrel (Oceanodroma tristrami), Amaranthus brownii, presumed extinct kamanomano (Cenchrus agrimoniodes var. laysanensis), Cyperus pennatiformis var. bryanii, lou'lu (Pritchardia remota), Schiedea verticillata, and 'ohai (Sesbania tomentosa) (PMNM, 2008; Friedlander and others, 2009). Additionally, the islands are winter habitat for regionally significant populations of four migratory shorebirds: Pacific Golden-plover or Kolea (Pluvialis fulva), Bristle-thighed Curlew or Kioea (Numenius tahitiensis), Wandering Tattler or 'Ulili (Tringa incana), and Ruddy Turnstone or 'Akekeke (Arenaria interpres; Engilis and Naughton, 2004; PMNM, 2008).

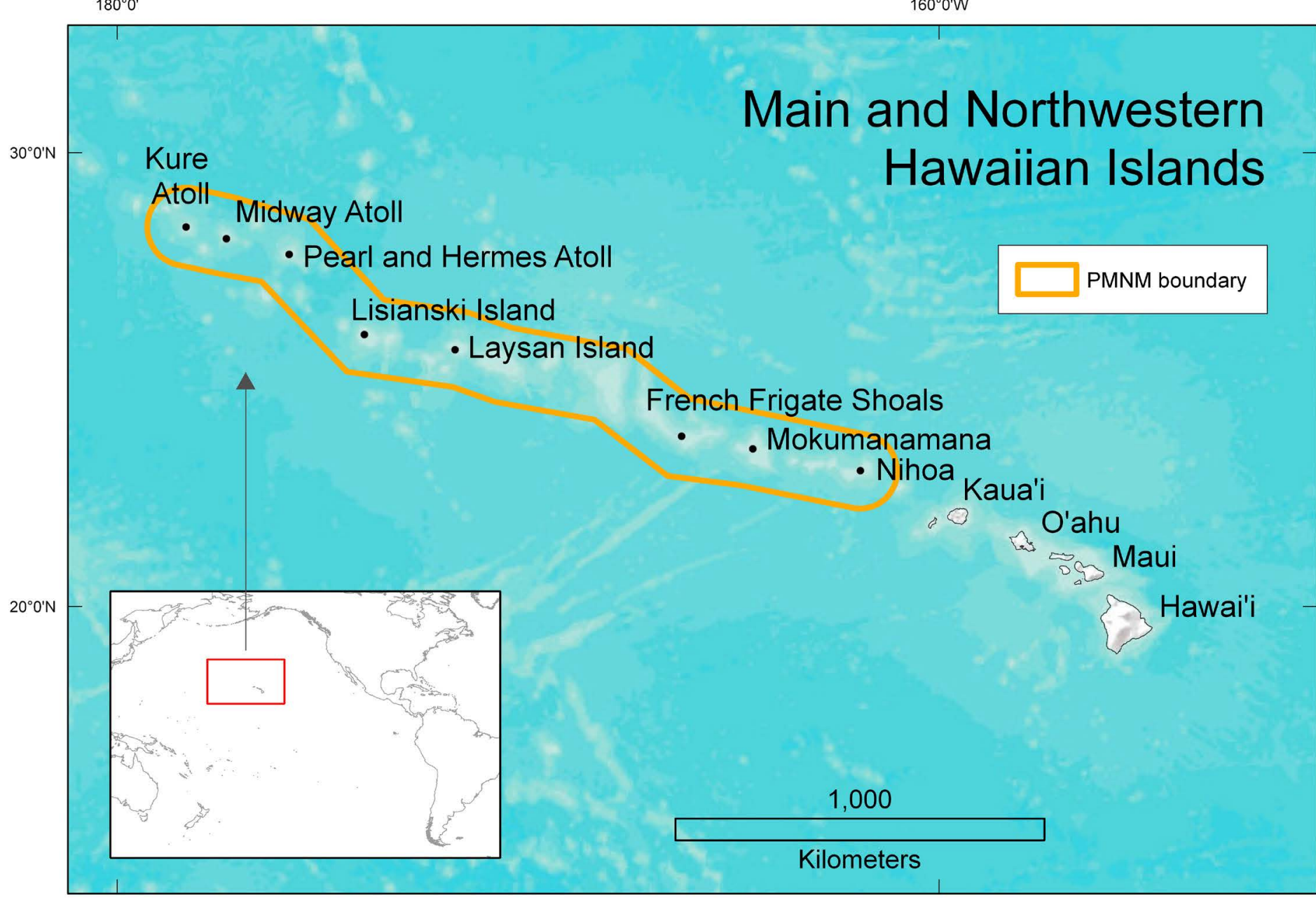

Figure 1.2. Map of the Hawaiian Archipelago with the main Hawaiian Islands (Kaua'i to Hawai'i) and Papahānaumokuākea Marine National Monument (PMNM; 362,061 square kilometers), the largest conservation area in the United States. 
Table 1.1. Known breeding distribution of avifauna, endangered plants, and other wildlife in the Northwestern Hawaiian Islands. [Data compiled from published and unpublished sources (USFWS data). See atoll- and island-specific footnotes for sources]

\begin{tabular}{|c|c|c|c|c|c|c|c|c|c|c|c|c|c|c|c|c|c|c|c|c|}
\hline \multirow[b]{2}{*}{ Species } & \multirow[b]{2}{*}{ Scientific name } & \multirow{2}{*}{ 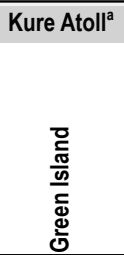 } & \multicolumn{3}{|c|}{ Midway Atoll } & \multicolumn{4}{|c|}{ Pearl and Hermes Atoll ${ }^{c, d}$} & \multirow[b]{2}{*}{ 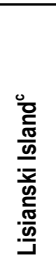 } & \multirow[b]{2}{*}{ 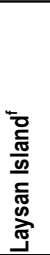 } & \multirow[b]{2}{*}{ 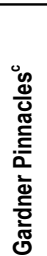 } & \multicolumn{6}{|c|}{ French Frigate Shoals $^{e}$} & \multirow[b]{2}{*}{ 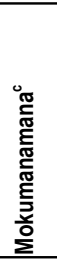 } & \multirow[b]{2}{*}{ 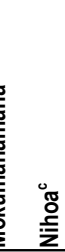 } \\
\hline & & & 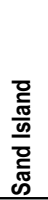 & 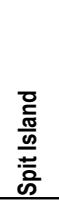 & 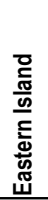 & 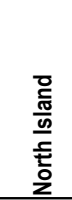 & 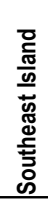 & 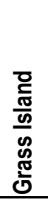 & 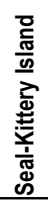 & & & & 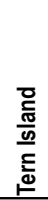 & 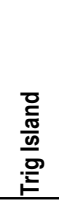 & 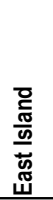 & 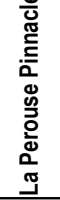 & 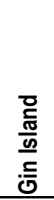 & 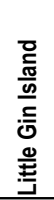 & & \\
\hline Black-footed Albatross & Phoebastria nigripes & $\mathrm{X}$ & $\mathrm{X}$ & $\mathrm{X}$ & $\mathrm{X}$ & $\mathrm{X}$ & $\mathrm{X}$ & $\mathrm{X}$ & $\mathrm{X}$ & $\mathrm{X}$ & $\mathrm{X}$ & & $\mathrm{X}$ & $\mathrm{X}$ & $\bar{x}$ & & $\mathrm{X}$ & $\mathrm{X}$ & $\mathrm{X}$ & $\mathrm{X}$ \\
\hline Laysan Albatross & Phoebastria immutabilis & $\mathrm{x}$ & $\mathrm{X}$ & $\mathrm{X}$ & $\mathrm{X}$ & $\mathrm{X}$ & $\mathrm{X}$ & $\mathrm{X}$ & $\mathrm{X}$ & $\mathrm{X}$ & $\mathrm{X}$ & $\mathrm{X}$ & $\mathrm{X}$ & $\mathrm{X}$ & $\mathrm{X}$ & & $\mathrm{X}$ & $\mathrm{X}$ & $\mathrm{X}$ & $\mathrm{X}$ \\
\hline Short-tailed Albatross & Phoebastria albatrus & & & & $\mathrm{X}$ & & & & & & & & & & & & & & & \\
\hline Bonin Petrel & Pterodroma hypoleuca & $\mathrm{X}$ & $\mathrm{X}$ & $\mathrm{X}$ & $\mathrm{X}$ & & $\mathrm{X}$ & $\mathrm{X}$ & $\mathrm{X}$ & $\mathrm{X}$ & $\mathrm{X}$ & & $\mathrm{X}$ & & $\mathrm{X}$ & & & & & \\
\hline Bulwer's Petrel & Bulweria bulwerii & $\mathrm{X}$ & & & & & $\mathrm{X}$ & & & $\mathrm{X}$ & $\mathrm{X}$ & $\mathrm{X}$ & $\mathrm{X}$ & & $\mathrm{X}$ & & & & $\mathrm{X}$ & $\mathrm{X}$ \\
\hline Wedge-tailed Shearwater & Puffinus pacificus & $\mathrm{X}$ & $\mathrm{X}$ & & $\mathrm{X}$ & & $\mathrm{X}$ & $\mathrm{X}$ & $\mathrm{X}$ & $\mathrm{X}$ & $\mathrm{X}$ & $\mathrm{X}$ & $\mathrm{X}$ & & $\mathrm{X}$ & & & & $\mathrm{X}$ & $\mathrm{X}$ \\
\hline Christmas Shearwater & Puffinus nativitatis & $\mathrm{x}$ & & & $\mathrm{X}$ & & $\mathrm{X}$ & $\mathrm{X}$ & $\mathrm{X}$ & $\mathrm{X}$ & $\mathrm{X}$ & & $\mathrm{X}$ & & $\mathrm{X}$ & & & & & $\mathrm{X}$ \\
\hline Tristram's Storm-petrel & Oceanodroma tristrami & $\mathrm{x}$ & & & & $\mathrm{X}$ & $\mathrm{X}$ & $\mathrm{X}$ & $\mathrm{X}$ & & $\mathrm{X}$ & & $\mathrm{X}$ & & $\mathrm{X}$ & & & & $\mathrm{X}$ & $\mathrm{X}$ \\
\hline Red-tailed Tropicbird & Phaethon rubricauda rubricauda & $\mathrm{x}$ & $\mathrm{X}$ & $\mathrm{X}$ & $\mathrm{X}$ & $\mathrm{X}$ & $\mathrm{X}$ & $\mathrm{X}$ & $\mathrm{X}$ & $\mathrm{X}$ & $\mathrm{X}$ & $\mathrm{X}$ & $\mathrm{X}$ & & $\mathrm{X}$ & & & & $\mathrm{X}$ & $\mathrm{X}$ \\
\hline White-tailed Tropicbird & Phaethon lepturus dorotheae & & $\mathrm{X}$ & & & & & & & & & & & & & & & & & \\
\hline Masked Booby & Sula dactylatra personata & $\mathrm{x}$ & & $\mathrm{X}$ & $\mathrm{X}$ & $\mathrm{X}$ & $\mathrm{X}$ & $\mathrm{X}$ & $\mathrm{X}$ & $\mathrm{X}$ & $\mathrm{X}$ & $\mathrm{X}$ & $\mathrm{X}$ & $\mathrm{X}$ & $\mathrm{X}$ & & $\mathrm{X}$ & $\mathrm{X}$ & $\mathrm{X}$ & $\mathrm{X}$ \\
\hline Brown Booby & Sula leucogaster plotus & $\mathrm{x}$ & & & $\mathrm{X}$ & & $\mathrm{X}$ & & & $\mathrm{X}$ & $\mathrm{X}$ & $\mathrm{X}$ & & & & $\mathrm{X}$ & & & $\mathrm{X}$ & $\mathrm{X}$ \\
\hline Red-footed Booby & Sula sula rubripes & $\mathrm{x}$ & & $\mathrm{X}$ & $\mathrm{X}$ & $\mathrm{X}$ & $\mathrm{X}$ & & & $\mathrm{X}$ & $\mathrm{X}$ & & $\mathrm{X}$ & & $\mathrm{X}$ & $\mathrm{X}$ & & & $\mathrm{X}$ & $\mathrm{X}$ \\
\hline Great Frigatebird & Fregata minor palmerstoni & $\mathrm{x}$ & & $\mathrm{X}$ & $\mathrm{X}$ & $\mathrm{X}$ & & $\mathrm{X}$ & & $\mathrm{X}$ & $\mathrm{X}$ & & $\mathrm{X}$ & & $\mathrm{X}$ & & & & $\mathrm{X}$ & $\mathrm{X}$ \\
\hline Little Tern & Sterna albifrons sinensis & & $\mathrm{X}$ & & & & $\mathrm{X}$ & & & & & & & & & & & & & \\
\hline Gray-backed Tern & Onychoprion lunatus & $\mathrm{X}$ & & $\mathrm{X}$ & $\mathrm{X}$ & & $\mathrm{X}$ & & $\mathrm{X}$ & $\mathrm{X}$ & $\mathrm{X}$ & $\mathrm{X}$ & $\mathrm{X}$ & & $\mathrm{X}$ & & & & $\mathrm{X}$ & $\mathrm{X}$ \\
\hline Sooty Tern & Onychoprion fuscata oahuensis & $\mathrm{X}$ & & & $\mathrm{X}$ & $\mathrm{X}$ & $\mathrm{X}$ & & & $\mathrm{X}$ & $\mathrm{X}$ & $\mathrm{X}$ & $\mathrm{X}$ & & & & & & $\mathrm{X}$ & $\mathrm{X}$ \\
\hline Blue Noddy & Procelsterna cerulean saxatilis & & & & & & & & & & & $\mathrm{X}$ & & & & $\mathrm{X}$ & & & $\mathrm{X}$ & $\mathrm{X}$ \\
\hline Brown Noddy & Anous stolidus pileatus & $\mathrm{X}$ & $\mathrm{X}$ & $\mathrm{X}$ & $\mathrm{X}$ & $\mathrm{X}$ & $\mathrm{X}$ & $\mathrm{X}$ & $\mathrm{X}$ & $\mathrm{X}$ & $\mathrm{X}$ & $\mathrm{X}$ & $\mathrm{X}$ & & $\mathrm{X}$ & $\mathrm{X}$ & $\mathrm{X}$ & & $\mathrm{X}$ & $\mathrm{X}$ \\
\hline Black Noddy & Anous minutus marcusi & $\mathrm{x}$ & $\mathrm{X}$ & & $\mathrm{X}$ & & $\mathrm{X}$ & & & $\mathrm{X}$ & $\mathrm{X}$ & $\mathrm{X}$ & $\mathrm{X}$ & & & & & & $\mathrm{X}$ & $\mathrm{X}$ \\
\hline White Tern & Gygis alba candida & $\mathrm{X}$ & $\mathrm{X}$ & & $\mathrm{X}$ & & $\mathrm{X}$ & & $\mathrm{X}$ & $\mathrm{X}$ & $\mathrm{X}$ & $\mathrm{X}$ & $\mathrm{X}$ & & & $\mathrm{X}$ & & & $\mathrm{X}$ & $\mathrm{X}$ \\
\hline Laysan Teal & Anas laysanensis & & $\mathrm{X}$ & & $\mathrm{X}$ & & & & & & $\mathrm{X}$ & & & & & & & & & \\
\hline Laysan Finch & Telespiza cantans & & & & & & $\mathrm{X}$ & & & & $\mathrm{X}$ & & & & & & & & & \\
\hline Nihoa Finch & Telespiza ultima & & & & & & & & & & & & & & & & & & & $\mathrm{X}$ \\
\hline Nihoa Millerbird ${ }^{\mathrm{g}}$ & Acrocephalus familiaris kingi & & & & & & & & & & & & & & & & & & & $\mathrm{X}$ \\
\hline Hawaiian monk seal & Monachus schauinslandi & $\mathrm{X}$ & $\mathrm{X}$ & $\mathrm{X}$ & $\mathrm{X}$ & $\mathrm{X}$ & $\mathrm{X}$ & $\mathrm{X}$ & $\mathrm{X}$ & $\mathrm{X}$ & $\mathrm{X}$ & & $\mathrm{X}$ & $\mathrm{X}$ & $\mathrm{X}$ & & $\mathrm{X}$ & $\mathrm{X}$ & $\mathrm{X}$ & $\mathrm{X}$ \\
\hline Hawaiian green turtle ${ }^{\mathrm{h}}$ & Chelonia mydas & $\mathrm{x}$ & $\mathrm{X}$ & $\mathrm{X}$ & $\mathrm{X}$ & $\mathrm{X}$ & $\mathrm{X}$ & $\mathrm{X}$ & $\mathrm{X}$ & $\mathrm{X}$ & $\mathrm{X}$ & & $\mathrm{X}$ & $\mathrm{X}$ & $\mathrm{X}$ & & $\mathrm{X}$ & $\mathrm{X}$ & & \\
\hline$*$ & Amaranthus brownii & & & & & & & & & & & & & & & & & & & $\mathrm{X}$ \\
\hline * & Cyperus pennatiformis var. bryanii & & & & & & & & & & $\mathrm{X}$ & & & & & & & & & \\
\hline *Lou'lu & Pritchardia remota & & & & & & & & & & & & & & & & & & & $\mathrm{X}$ \\
\hline * & Schiedea verticillata & & & & & & & & & & & & & & & & & & & $\mathrm{X}$ \\
\hline *`Ōhai & Sesbania tomentosa & & & & & & & & & & & & & & & & & & $\mathrm{X}$ & $\mathrm{X}$ \\
\hline
\end{tabular}

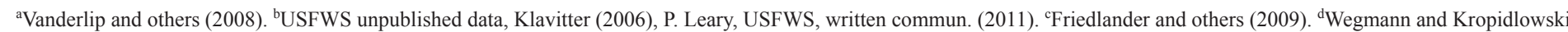
(2002). ${ }^{\mathrm{e}}$. Hartzell, USFWS, written commun. (2011). ${ }^{\mathrm{f}} \mathrm{Hammond}$ and others (2010), Kristof and others (2011). ${ }^{\mathrm{g}}$ Twenty-four individuals translocated to Laysan Island in September 2011

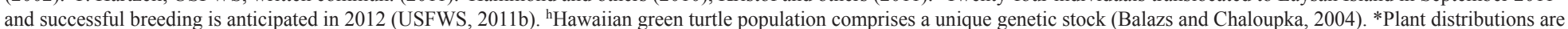
of extant populations and do not include outplantings and introductions on other islands. [USFWS, U.S. Fish and Wildlife Service] 


\section{Kure Atoll (Mokupāpapa) 28 $23^{\prime} 33^{\prime \prime} \mathrm{N}$ and $178^{\circ} 17^{\prime} 36^{\prime \prime} \mathrm{W}$}

Kure is the northernmost coral atoll in the world, the westernmost land mass of the NWHI, and approximately $2,400 \mathrm{~km}$ northwest of Honolulu. The nearest land is Sand Island, Midway Atoll, $92 \mathrm{~km}$ to the southeast. Kure Atoll includes Green Island and several small sand spits. Seventeen seabird species breed at Green Island (table 1.1), excluding the single pair of endangered Short-tailed Albatross, which has not bred successfully yet (C. Vanderlip, Hawai'i Department of Land and Natural Resources [DLNR], written commun., 2011).

Kure Atoll was visited by feather hunters during the 19th century and is the site of numerous shipwrecks from that era (Woodward, 1972). The U.S. Coast Guard began construction of a long-range navigation (loran) radar station in 1960; the station was in use until 1992 (Rauzon, 2001). In 1981, Kure was designated a State wildlife sanctuary. In 1993 the Hawai' $i$ DLNR began restoration projects including the eradication of Polynesian rats (Rattus exulans; Rauzon, 2001), a predator of Hawaiian birds and plants (Kepler, 1967).

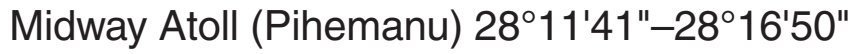 $\mathrm{N}$ and $177^{\circ} 18^{\prime} 38^{\prime \prime}-177^{\circ} 25^{\prime} 38^{\prime \prime} \mathrm{W}$}

Midway is a coral atoll situated between Kure Atoll to the northwest and Pearl and Hermes Atoll to the southeast. Midway Atoll consists of three islands: Sand, Spit, and Eastern. Eighteen seabird species and a reintroduced population of endangered Laysan Teal breed at Midway Atoll (table 1.1). Midway Atoll is the only location in the NWHI where the endangered Short-tailed Albatross has bred successfully (USFWS, 2011a). Two additional seabird species (Bulwer's Petrel [Bulweria bulwerii] and Tristram's Storm-petrel) are recolonizing Midway, but nesting has not been confirmed (P. Leary, USFWS, written commun., 2011). Additionally, the current status of Bryan's Shearwater (Puffinus bryani), a species newly described from a specimen first collected at Midway Atoll in 1963, is unknown (Pyle and others, 2011).

Sand and Eastern Islands have been significantly altered by human activity since the early 1900s (McDermond and Morgan, 1993). Midway has been occupied since coming under the jurisdiction of the U.S. in 1903 (Bryan, 1938). As a national defense area, significant development of the islands occurred, including the addition of seawalls, a harbor, piers, runways, dredge and fill operations, numerous buildings, and species introductions between the 1930s and 1990s (SpeuldaDrews, 2010). In 1993 the naval air station was decommissioned, and in 1996 jurisdiction was transferred to the U.S. Department of the Interior as a National Wildlife Refuge (Speulda-Drews, 2010). Introduced black rats (Rattus rattus), predators of Hawaiian birds and plants (Fisher and Baldwin, 1946), were eradicated by 1997 (Rauzon, 2001).

\section{Pearl and Hermes Atoll (Holoikauaua) $27^{\circ} 45^{\prime} 52^{\prime \prime}-27^{\circ} 58^{\prime} 36^{\prime \prime} \mathrm{N}$ and $175^{\circ} 42^{\prime} 05^{\prime \prime}-$ $176^{\circ} 02^{\prime} 11^{\prime \prime} \mathrm{W}$}

Pearl and Hermes is a coral atoll situated between Midway Atoll (160 km to the northwest) and Lisianski Island (265 km to the southeast). Pearl and Hermes Atoll currently comprises five islands: North, Little North (made up of two sections separated by water), Southeast, Grass, and SealKittery, plus several sand spits. Eighteen seabird species and a translocated population of endangered Laysan Finch breed at Pearl and Hermes Atoll (table 1.1).

As on the other atolls, Pearl and Hermes has a history of human impacts. The extent of human disturbance from feather collecting and guano mining in the early 1900s at Pearl and Hermes is not clear, although there is evidence of human occupation and rabbit (Oryctolagus cuniculus) introduction and subsequent extirpation (Wetmore 1923 unpub. personal journal reproduced in Olson, 1996). In 1927, black-lip pearl oysters were found in the atoll and 150 tons were removed in three years; by 1969 only one oyster could be found (Amerson and others, 1974). During World War II, a small U.S. Navy encampment occupied Pearl and Hermes Atoll (Amerson and others, 1974). Several species translocations have been made at Pearl and Hermes, with failed establishment of Laysan Rail in 1929 (Baldwin, 1945), Laysan Teal in 1967, and successful establishment of Laysan Finch 1967 (Amerson and others, 1974).

\section{Lisianski Island (Papa'āpoho) 2603'48" N and $173^{\circ} 57^{\prime} 56^{\prime \prime} \mathrm{W}$}

Lisianski, the third largest island within the monument, is a raised atoll situated between Pearl and Hermes Atoll and Laysan Island. Sixteen seabird species breed at Lisianski Island (table 1.1). Early disturbances at Lisianski included multiple shipwrecks (Ward, 1967), guano mining (Bryan, 1942), and feather collecting (Clapp and Wirtz, 1975; Olson and Ziegler, 1995). The introduction of mice (Mus musculus) in the $1800 \mathrm{~s}$ and later rabbits destroyed much of the flora and fauna on the island (Wetmore 1923 unpub. personal journal reproduced in Olson, 1996) until de-vegetation resulted in the die-off of the mammals. The mammal-caused habitat destruction and hungry shipwrecked mariners contributed to the extirpation of the Laysan Teal population at Lisianski (Olson and Ziegler, 1995).

\section{Laysan Island (Kauō) $25^{\circ} 46^{\prime} 11^{\prime \prime} \mathrm{N}$ and $171^{\circ} 44^{\prime} 00^{\prime \prime} \mathrm{W}$}

Laysan is situated between Lisianski Island to the northwest and Maro Reef to the southeast; a detailed description of Laysan Island is provided in chap. 2. Laysan lies approximately $1,600 \mathrm{~km}$ northwest of Honolulu, has 17 seabird species and 3 resident endangered land birds: Laysan Teal, Laysan Finch, and a newly (2011) translocated population of Nihoa Millerbird 
(USFWS, 2011b; table 1.1). The endemic endangered plant Cyperus pennatiformis var. bryanii is found on Laysan Island.

Guano miners in the early 1900s introduced rabbits and other mammals to Laysan Island; these mammals devastated the flora and fauna (Wetmore 1923 unpub. personal journal reproduced in Olson, 1996). The Tanager Expedition in 1923 reduced the rabbit population and documented the island as barren (Wetmore, 1925). Soon after the expedition the rabbits and other introduced mammals at Laysan died off from starvation (Wetmore, 1925). Laysan has been protected as a bird reserve since 1909 .

\section{French Frigate Shoals (Kānemiloha'i) $23^{\circ} 37^{\prime} 18^{\prime \prime}-23^{\circ} 52^{\prime} 50^{\prime \prime} \mathrm{N}$ and $166^{\circ} 03^{\prime} 14^{\prime \prime}-$ $166^{\circ} 20^{\prime} 04^{\prime \prime} \mathrm{W}$}

French Frigate Shoals is an open atoll located near the midpoint of the Hawaiian archipelago. French Frigate Shoals is approximately $975 \mathrm{~km}$ northwest of Honolulu. The nearest land, Mokumanamana, lies $168 \mathrm{~km}$ to the southeast. French Frigate Shoals currently comprises nine islands: Shark, Tern, Trig, Round, East, La Perouse Pinnacle (a basalt rock formation), Gin, Little Gin, and Disappearing. Currently, 16 seabird species breed at Tern Island, 13 at East Island, 6 at La Perouse Pinnacle, 4 at Gin Island, and 3 at Trig and Little Gin islands (table 1.1). Although green turtles are distributed in tropical oceans globally (Bowen and others, 1992), 90 percent of the Hawaiian subpopulation nests at French Frigate Shoals, and of those, 50 percent nest at East Island (Balazs, 1976; Balazs and Chaloupka, 2004; Tiwari and others, 2010).

The islands of French Frigate Shoals were not subjected to guano mining and feather collecting, but were occupied by the U.S. military during World War II and served as a loran radar station from 1944-1979 (Amerson, 1971; Friedlander and others, 2009). The U.S. Coast Guard decommissioned Tern Island and transferred management of the island to the USFWS in 1979 (Rauzon, 2001). An unsuccessful translocation of Nihoa Finch occurred in 1967 (Amerson, 1971).

\section{Mokumanamana (Necker Island) $23^{\circ} 34^{\prime} 32^{\prime \prime} \mathrm{N}$ and $164^{\circ} 42^{\prime} 02^{\prime \prime} \mathrm{W}$}

Mokumanamana is a small basalt island (18.6 ha) with several peaks higher than $70 \mathrm{~m}$; the highest peak is $82 \mathrm{~m}$ (Evenhuis and Eldredge, 2004). Mokumanamana is approximately $750 \mathrm{~km}$ northwest of Honolulu. The nearest land is to the north at French Frigate Shoals. The island is known for its religious and cultural importance, linking early Tahitian and Hawaiian cultures (Kikiloi, 2010). Sixteen seabird species breed at Mokumanamana (table 1.1).

\section{Nihoa $23^{\circ} 3^{\prime} 36^{\prime \prime} \mathrm{N}$ and $161^{\circ} 55^{\prime} 24^{\prime \prime} \mathrm{W}$}

Nihoa is a basalt rock island of 63.1 ha at the southernmost end of the NWHI chain. Nihoa is approximately 250 $\mathrm{km}$ northwest of Honolulu. The nearest land is Kaula Island, near Kaua'i. Two high cliffs of Nihoa, which reach $269 \mathrm{~m}$ and $256 \mathrm{~m}$, are unique topographically among the NWHI (Evenhuis and Eldredge, 2004). Nihoa was colonized by Hawaiians between A.D. 1000 and A.D. 1700 and has more than 80 known culturally significant sites (Evenhuis and Eldredge, 2004). Seventeen seabird species; 2 endangered land birds, Nihoa Finch and Nihoa Millerbird; and 4 endangered plants, Amaranthus brownii, Pritchardia remota, Schiedea verticillata, and Sesbania tomentosa, are found on Nihoa (table 1.1).

La Perouse Pinnacle, Gardner Pinnacles, and the islands of Mokumanamana and Nihoa were not included in the SLR assessments because of their high elevation and low vulnerability to inundation.

\section{Remote Sensing Data}

\section{Light Detection and Ranging (lidar)}

Lidar is an active remote sensing technique that measures the length of time between transmitted laser pulses and the detection of their reflected signals (USGS, 2010). The length of time is converted to a distance value that allows us to determine the elevation of any targeted surface (for example, bare earth, vegetation canopy). Although lidar sensors may be mounted on various objects (fixed-wing aircraft, helicopters, watercraft, tripods on the ground, etc.), the size, vast distances, and ecological fragility within the project area warranted an airborne application. We collected terrestrial lidar data of the study area using a Beechcraft Queen Air twin engine plane (Aerial Surveying, Inc.) that departed from Kaua' $i$ Island and then based survey flights from Midway Atoll National Wildlife Refuge (NWR) July 22-25, 2010 (table 1.2).

\section{Lidar Data Collection}

Two Riegl sensors, models LMS-Q140i and LMS-Q240, were used to acquire more than 20,000 laser pulses per second with a ground scan swath of $400 \mathrm{~m}$ in a downward direction below the aircraft (fig. 1.3). The instrument settings yielded a pulse density of approximately 2 points per square meter $\left(\mathrm{m}^{2}\right)$. Aircraft position heading, pitch, and roll were measured 200 times per second (200 hertz, Hz) from an onboard inertial measurement unit (IMU). Our study design specifications for the lidar data collection are given in appendix 1. A Global Positioning System (GPS) based ground survey, comprising 1,678 real-time kinematic points on Sand Island (Midway Atoll), occurred concurrently with the airborne lidar data collection. The lidar data points were processed and tested for accuracy against the real-time kinematic survey points.

\section{Lidar Data Processing}

During data processing and analysis, lidar points above the ground surface were removed, creating a comprehensive 
Table 1.2. Northwestern Hawaiian Islands airborne lidar data collection information, including root-mean-squared error (RMSE) and standard deviation (SD) values measured between ground survey control points and lidar points.

[The 80-percent (\%) confidence interval refers to the digital elevation model vertical accuracy]

\begin{tabular}{lcccccc}
\hline & Date & Hawaii Standard Time & $\begin{array}{c}\text { RMSE } \\
\text { (meters) }\end{array}$ & $\begin{array}{c}\text { Mean } \\
\text { (meters) }\end{array}$ & SD (meters) & $\begin{array}{c}\mathbf{8 0 \%} \\
\text { Confidence } \\
\text { interval }\end{array}$ \\
\hline Kure Atoll & July 23, 2010 & $1118-1207$ & 0.11 & 0.08 & 0.09 & 0.20 \\
Midway Atoll & July 23, 2010 & $1230-1457$ & 0.05 & 0.00 & 0.05 & 0.09 \\
Pearl and Hermes Atoll & July 25, 2010 & $1205-1246$ & 0.12 & -0.10 & 0.06 & 0.22 \\
Lisianski Island & July 24, 2010 & $1636-1705$ & 0.18 & 0.00 & 0.18 & 0.32 \\
Laysan Island & July 24, 2010 & $1436-1505$ & 0.09 & 0.00 & 0.09 & 0.16 \\
French Frigate Shoals & July 22, 2010 & $1200-1330$ & 0.05 & 0.00 & 0.05 & 0.09 \\
\hline
\end{tabular}

${ }^{a}$ Hawaii Standard Time is the standard time in the 10th time zone west of Greenwich, reckoned at the 150th meridian west; used in Hawai $i$ and the western Aleutian Islands. Times are given using a 24-hour clock.

data set of points from various surfaces (for example, bare earth, vegetation, buildings) to be classified. Automated identification of points was done using MARS 7 software (Merrick, 2011); manual removal of outliers followed and consisted of visual inspection and editing, particularly in areas where the automated methodology was known to be deficient (for example, dense vegetation). First-return points and the ground-classified points were used to create separate triangulated irregular networks (TINs) that were then converted into two $1.0-\mathrm{m}$ cell size (pixel grid) digital elevation models (DEMs), a first-return DEM and a bare-earth DEM. We used the North American Datum of 1983 (NAD83) as the horizontal datum and the Universal Transverse Mercator (UTM), zones $1-3$, as the coordinate system. Elevation data were provided relative to mean sea level (MSL) in meters.

\section{Lidar Quality Assessment/Quality Control}

An accuracy assessment was conducted to compare known points on the ground with those points generated from our lidar data (unpub. data, NOAA, 2011a). A dual-frequency L1/L2 Thales Z-Max GPS receiver was used as a base station. The base station was set up on existing geodetic markers when possible and collected data continuously throughout each day of survey activity. Two single-frequency L1 ProMark 3 GPS receivers were used as roving instruments to collect elevation data points. A minimum of 20 points were surveyed on each island or atoll area (in the case of the smaller atoll islets) to allow for a statistically rigorous analysis of accuracy. The locations selected for the GPS survey points met the following criteria: (1) flat, bare ground at least $4 \mathrm{~m}$ in diameter, (2) absence of tall vegetation within $5 \mathrm{~m}$ of the point, and (3) stable ground in areas assumed to be unaffected by the severe winter 2010-11 storms or the March 11, 2011 tsunami (USFWS, 2011c).

The data were then processed for the lidar accuracy assessment. The GPS data were imported into Ashtech's GNSS Solutions software (version 3.60.1) for post-processing and data adjustment. The GPS base station locations were processed through the online positioning user service (OPUS) of the National Geodetic Survey (NGS) to obtain both NAD83 and International Terrestrial Reference Frame of 2000 (ITRF00) positions. The ITRF00 ellipsoid heights were used if known heights were not available. The two-step data processing produced corrected positions for the base station points and adjusted positions for the roving points. The OPUS-derived base station coordinates were entered in GNSS

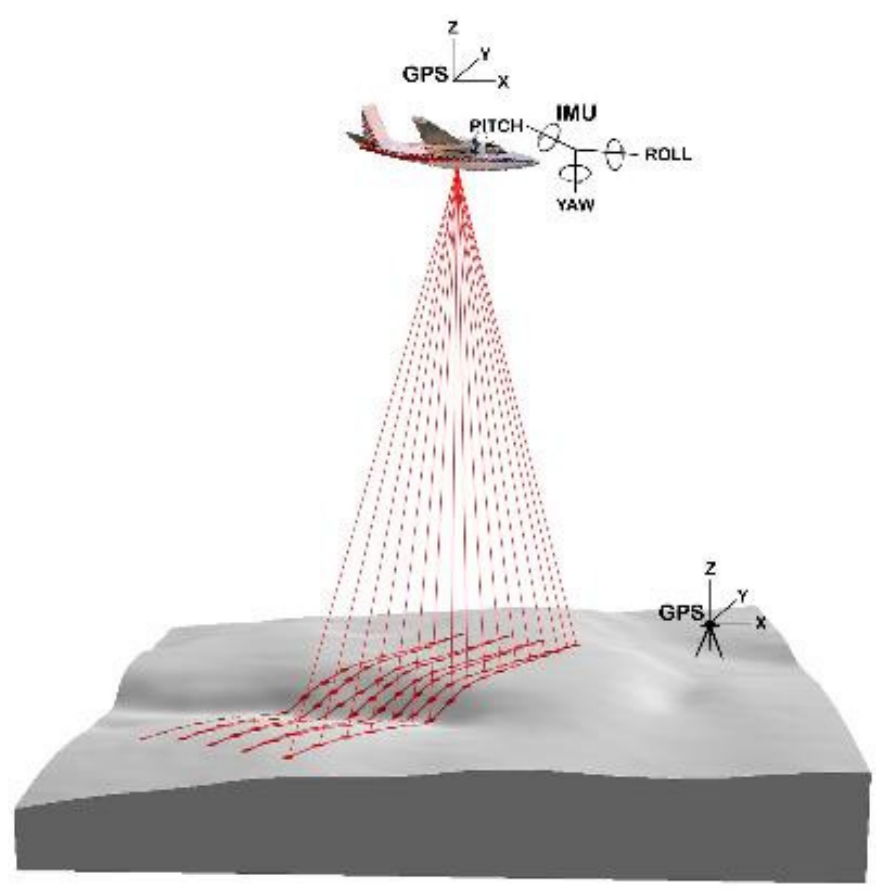

Figure 1.3. Schematic showing the lidar (light detection and ranging) data collection process, which combines a Global Positioning System (GPS), an inertia measurement unit (IMU), and light pulses from a laser. Lidar data are often collected from aircraft to allow for a rapid collection of points (more than 70,000 per second) over a large spatial area (reproduced from U.S. Department of Agriculture Forest Service, http://forsys.cfr. washington.edu/JFSP06/lidar_technology.htm). 
Solutions to assign their positions and allow the roving points to adjust accordingly.

Each position was also determined in relation to two vertical datums: NAD83/GRS80 and WGS84. OPUS was used to generate fixed positions for the base stations. These positions were compared against known geodetic controls where available. In those cases, the published NGS datasheet of precise latitude and longitude positions were processed using NGS' Horizontal Time Dependent Positioning (HTDP) tool to update the positions with known velocities. The resulting positions included information about the region, island, latitude, longitude, UTM zone, and ellipsoid heights (GRS80 and WGS84).

Data accuracy was derived from the absolute root-meansquared error (RMSE), a statistical measure of variability similar to standard deviation. In a nonbiased data set the two values will be the same (NOAA, 2009). RMSE is calculated directly from the differences between the ground control points and the lidar ellipsoidal elevations (table 1.2).

\section{Aerial and Satellite Imagery}

We acquired aerial imagery for the NWHI from multiple sources (table 1.3). All images were georeferenced to NAD83 as the horizontal datum and UTM zones $1-3$ as the coordinate system. We purchased Digital Globe QuickBird and WorldView-2 satellite imagery (Digital Globe Inc., 2010). Lastly, we acquired additional WorldView-2 stereopair imagery from PhotoSat Information Ltd. (2010) for Laysan (also see chap. 2), Trig, Little Gin, Round Islands, and La Perouse Pinnacle. PhotoSat also provided DEMs utilizing a geophysical processing system (Mitchell and MacNabb, 2010); these DEMs supplemented our lidarderived DEMs.

\section{Climate Data}

To better understand possible climate impacts for the NWHI, we analyzed future climates from general circulation models (GCMs) with interpolated climate surfaces of baseline data (Tabor and Williams, 2010). There are a number of different GCMs, and emission scenarios for future atmospheric concentrations of greenhouse gasses. Thus, projected
Table 1.3. Satellite imagery sources and collection dates. [All images georeferenced to NAD 83, UTM zones 1-3]

\begin{tabular}{lcc}
\hline & $\begin{array}{c}\text { Digital Globe } \\
\text { QuickBird }\end{array}$ & WorldView-2 \\
\hline Kure Atoll & July 2008 & - \\
Midway Atoll & March 2009 & August 2011 \\
Pearl and Hermes Atoll & October 2007 & December 2010 \\
Lisianski Island & January 2008 & March 2011 \\
Laysan Island & January 2009 & May 2010 \\
French Frigate Shoals & November 2005 & August 2011 \\
\hline
\end{tabular}

climate for a given period in the future depends on the GCM model and emission scenario used. We used six GCMs and scenarios from the IPCC AR4 (2007; table 1.4) appropriate for the Hawaiian Islands. Timm and Diaz (2009) favored these models for accurate representation of inter-annual to decadal variability of precipitation patterns around the main Hawaiian Islands and extra-tropical to tropical teleconnection regions (distant areas influenced by the same climate anomalies).

Due to the coarse resolution (approximately $300-\mathrm{km}$ cell size) of GCM data, we "downscaled" our data by computing the absolute difference between GCM output and baseline data (Tabor and Williams, 2010). The application of downscaling calibrates climate data to a resolution appropriate for the NWHI. These downscaled values were then applied to WorldClim data, high-resolution (1-km cell size) interpolated climate surfaces, derived from monthly averages of temperature and precipitation from a large number of global, regional, national, and local weather sources, and presented as a 30 -year average (1960-1990). These data sources are available through the WorldClim database (see http://www.worldclim. org; Hijmans and others, 2005). We present results for the predicted change in the annual average maximum temperature and total annual precipitation in 2100 .

\section{Sea-Level Rise}

We used our lidar-derived DEMs (1-m cell size) as elevation grids for the land areas of Kure Atoll, Midway Atoll, Pearl and Hermes Atoll, Lisianski Island, Laysan Island, and French Frigate Shoals (Shark, Tern, Gin, and Disappearing Islands).

Table 1.4. Climate change general circulation models used to predict temperature and precipitation through 2100 (Intergovernmental Panel on Climate Change, IPCC, 2007).

\begin{tabular}{ll}
\hline \multicolumn{1}{c}{ Agency } & \multicolumn{1}{c}{ Model name } \\
\hline Canadian Centre for Climate Modeling and Analysis, Canada & CCCMA CGCM3.1.T63 \\
Geophysical Fluid Dynamics Laboratory, National Oceanic and Atmospheric Administration, United States & GFDL CM2.0 \\
Geophysical Fluid Dynamics Laboratory, National Oceanic and Atmospheric Administration, United States & GFDL CM2.1 \\
Max Planck Institute for Meteorology, Germany & MPI ECHAM5 \\
Meteorological Research Institute, Japan Meteorological Agency, Japan & MRI CGCM2.3.2A \\
Hadley Centre for Climate Prediction and Research, Met Office, United Kingdom & UKMO HADCM3 \\
\hline
\end{tabular}


Table 1.5. National Oceanic and Atmospheric Administration tide values (http://tidesandcurrents.noaa.gov/data) for each atoll, expressed relative to mean lower low water, used to calculate mean high water (MHW).

[Where possible, verified tides were used to determine the tide at the time of data collection; otherwise predicted tides, scaled to the Honolulu tide gauge, were used. All values in meters]

\begin{tabular}{lcccc}
\hline & $\begin{array}{c}\text { MHW from } \\
\text { benchmark }\end{array}$ & $\begin{array}{c}\text { Tide at time } \\
\text { of lidar }\end{array}$ & $\begin{array}{c}\text { Lidar sea } \\
\text { level }\end{array}$ & Lidar MHW \\
\hline Kure Atoll & 0.4 & 0.2 & 0.0 & 0.2 \\
Midway Atoll & 0.4 & 0.3 & 0.0 & 0.1 \\
Pearl and Hermes Atoll & 0.4 & 0.3 & 0.0 & 0.1 \\
Lisianski Island & 0.2 & 0.3 & 0.0 & -0.1 \\
Laysan Island & 0.2 & 0.1 & 0.0 & 0.1 \\
French Frigate Shoals & 0.3 & 0.2 & 0.0 & 0.2 \\
\hline
\end{tabular}

For islands of French Frigate Shoals without lidar data (Trig, East, Round, Little Gin, and La Perouse Pinnacle), we used satellite-derived DEMs (Mitchell and MacNabb, 2010; PhotoSat Information Ltd., 2010). To model inundation from SLR, we used a passive inundation model to inundate grid cells below an elevation threshold, while accounting for connectivity to a water source ( $\mathrm{Li}$ and others, 2009), generally the ocean or, in the case of Laysan, the interior lake. Specifically, only those cells connected by an eight-neighbor relationship to the ocean (or interior lake) were inundated. By applying this constraint, lowlying elevations below a given threshold do not flood unless they are hydrologically connected to a water source. More complex and dynamic models that incorporate wave run-up require detailed bathymetric data that were not available for all islands. We describe a dynamic wave-driven inundation model for Laysan Island in chap. 2.

Our models did not predict ecological wetland succession or incorporate wetland response to projected changes in precipitation. However, for the interior lake of Laysan Island, we modeled SLR using two different lake (i.e., groundwater) responses: (1) no change in lake level or groundwater, and (2) a concurrent rise in groundwater equal to SLR. Under the latter scenario, if sea level rises $+1.0 \mathrm{~m}$, we predicted groundwater would also rise $+1.0 \mathrm{~m}$.

We used ArcGIS (version 10.0; ESRI 2010) to model four scenarios of SLR: $+0.5,+1.0,+1.5$, and $+2.0 \mathrm{~m}$. We referenced each SLR scenario to mean high water (MHW, table 1.5), which was based on NOAA tidal predictions from the nearest available data collection station or nearest location with predicted tidal data (http://tidesandcurrents.noaa. gov/tide_predictions). Sand Island at Midway Atoll has the only tide gauge in the NWHI with a long-term time series of tide data. Tidal predictions from Midway Atoll (based on the tidal epoch 1983-2001; NOAA Station ID 1619910 Sand Island, Midway Islands) were used for Midway, Kure, and Pearl and Hermes atolls. Tidal predictions (NOAA Station ID 1619645, scaled from the Honolulu tide gauge, and NOAA Station ID 1612340) were used for the islands of Laysan and Lisianski. For all islands in French Frigate Shoals, we used tide predictions from East Island (NOAA Station ID 1619222, scaled from the Honolulu tide gauge, NOAA Station ID 1612340).

\section{Land Cover Classification}

We classified land cover for Kure Atoll, Midway Atoll, Pearl and Hermes Atoll, Lisianski Island, and French Frigate Shoals using QuickBird satellite imagery collected during 2005-2009; for Laysan Island, we used WorldView-2 satellite imagery from 2010 (Digital Globe 2011; table 1.3). To classify satellite imagery digitally, we used the IsoCluster unsupervised classification tool in ArcGIS (ESRI, 2010); this tool classifies pixels based on color, texture, tone, pattern, and associated information (Xie and others, 2008). Where speciesspecific plant delineations were available from USFWS or Hawai' $i$ DLNR, we incorporated these data into vegetation maps (HI DLNR unpub. data, USFWS unpub. data, Vanderlip and others, 2007; Cornett and others, 2008; Vanderlip and others, 2008; Boyd and others, 2009; Kristof and others, 2011). Verbesina encelioides distribution was mapped and tabulated from field data collected in 2007 at Green Island, Kure Atoll (Vanderlip and others, 2007); 2004 at Sand and Eastern Islands, Midway Atoll (Laniawe, 2004); and 2003 at Southeast Island, Pearl and Hermes Atoll (Sprague, 2003). However, active management may influence the distribution of invasive species from year to year.

We classified land cover across the NWHI into 17 categories (table 1.6). General categories include a combination of vegetation classes (tree/shrub, mixed shrub, grass/herbaceous cover, vine/ground cover, wetland vegetation, and partially vegetated former runways), unvegetated areas (mudflats, standing water, bare ground, hard pan, and beach), and human infrastructure. Species-specific vegetation categories include three tree species (Casuarina equisetifolia, Cocos nucifera, and Tournefortia argentea), one shrub (Pluchea indica), and one herbaceous invasive plant (Verbesina encelioides). Verbesina encelioides is a dynamic and pervasive plant distributed 
Table 1.6. Land cover class definitions with plant species for each class.

[General description used to identify and delineate each land cover class from satellite image analysis. Land cover was classified from QuickBird (Kure Atoll [July 2008], Midway Atoll [March 2009], Pearl and Hermes Atoll [October 2007], Lisianski Island [January 2008], French Frigate Shoals [November 2005]), and WorldView-2 (Laysan Island [May 2010]) satellite imagery using IsoCluster methods (ESRI, 2011). Plant species were described from Wagner and others (2005) and multiple botanical reviews: Kure Atoll (Starr and others, 2001; Vanderlip and others, 2007; Vanderlip and others, 2008), Midway Atoll (Starr and Martz, 1999a; Klavitter, 2006; Starr and others, 2006; Starr and others, 2008), Pearl and Hermes Atoll (Starr and Martz, 1999a), Lisianski Island (Starr and Martz, 1999a), Laysan Island (Starr and Martz, 1999a; Kristof and others, 2011), and French Frigate Shoals (Starr and Martz, 1999a)]

\begin{tabular}{|c|c|c|}
\hline Land cover class & General description & Species included \\
\hline Tree/shrub & Continuous tree or shrub cover, multiple native and non-native species of 2-20 meters tall & $\begin{array}{l}\text { Capparis sandwichiana, Casuarina equisetifolia, Chenopodium oahuense, } \\
\text { Coccoloba uvifera, Cocos nucifera, Hibiscus tiliaceus, Lepidium } \\
\text { bidentatum o-waihiense, Pisonia grandis, Pluchea indica, Scaevola } \\
\text { taccada, Terminalia catappa, Tournefortia argentea }\end{array}$ \\
\hline Casuarina equisetifolia & Non-native tree species $10-20$ meters tall & Casuarina equisetifolia \\
\hline Cocos nucifera & Polynesian tree species with potential habitat in understory & Cocos nucifera \\
\hline Pluchea spp. & Non-native invasive shrub species up to 2 meters tall, Hawaii noxious weed designation & Pluchea indica, Pluchea carolinensis \\
\hline Tournefortia argentea & Polynesian coastal tree species with broad spreading crown & Tournefortia argentea \\
\hline Mixed shrub & Low-density shrub cover, multiple native and non-native species & $\begin{array}{l}\text { Capparis sandwichiana, Casuarina equisetifolia, Chenopodium oahuense, } \\
\text { Coccoloba uvifera, Cocos nucifera, Hibiscus tiliaceus, Lepidium } \\
\text { bidentatum o-waihiense, Pisonia grandis, Pluchea indica, Scaevola } \\
\text { taccada, Terminalia catappa, Tournefortia argentea }\end{array}$ \\
\hline Grass/herbaceous cover & $\begin{array}{l}\text { High-density grass and herbaceous cover, multiple native and non-native species, some speices up } \\
\text { to } 1.5 \text { meters tall }\end{array}$ & $\begin{array}{l}\text { Bidens alba, Cenchrus echinatus, Chamaesyce spp., Chenopodium murale, } \\
\text { Cynodon dactylon, Digitaria ciliaris, Eleusine indica, Eragrostis paupera, } \\
\text { Eragrostis variabilis, Euphorbia heterophylla, Fimbristylis cymosa, } \\
\text { Lepturus repens, Lobularia maritima, Malva parviflora, Pseudognaphalium } \\
\text { sandwicensium, Psilotum nudum, Setaria verticillata, Solanum } \\
\text { americanum, Solanum nelsonii, Sonchus oleraceus, Verbesina encelioides }\end{array}$ \\
\hline Verbesina encelioides & Non-native invasive erect annual herb, 0.3 to 1.7 meters & Verbesina encelioides \\
\hline Vine/ground cover & Low-lying vines and low herbaceous groundcover, multiple native and non-native species & $\begin{array}{l}\text { Vine: Boerhavia repens, Cassytha filiformis, Ipomoea indica, Ipomoea pes- } \\
\text { caprae, Sicyos maximowiczii, Sicyos pachycarpus; Ground cover: } \\
\text { Coronopus didymus, Heliotropium procumbens, Nama sandwicensis, } \\
\text { Portulaca lutea, Portulaca oleracea, Tribulus cistoides }\end{array}$ \\
\hline Wetland vegetation & Wetland plant species surviving in saturated conditions & $\begin{array}{l}\text { Batis maritima, Capparis sandwichiana, Cyperus javanicus, Cyperus } \\
\text { laevigatus, Cyperus pennatiformis var. bryanii, Cyperus polystachyos, } \\
\text { Heliotropium currasavicum, Pluchea indica, Sesuvium portulacastrum }\end{array}$ \\
\hline Partially vegetated former runway ${ }^{\mathrm{a}}$ & Dilapidated runway with some vegetation & Fimbristylis cymosa, Tribulus cistoides, Verbesina encelioides, \\
\hline Bare ground & Bare ground inland of beach area (without wave swash) & Unvegetated \\
\hline Hard pan & Former guano mining site with minimal vegetation & Portulaca spp., Sporobolus pyramidatus, Fimbristylis cymosa \\
\hline Beach & Coastal land subject to wave swash under typical conditions, typically sand, coral or rock & Unvegetated \\
\hline Wetland (unvegetated) & Primarily unvegetated mudflats & Unvegetated \\
\hline Wetland (standing water) & Perennially inundated area & Unvegetated \\
\hline Human structures $^{\mathrm{c}}$ & Runways, buildings, roads, seawalls, etc. & Unvegetated \\
\hline
\end{tabular}

${ }^{a}$ Only present on Green Island, Kure Atoll, and Eastern Island, Midway Atoll.

${ }^{b}$ Only present on Laysan Island. ${ }^{c}$ Buildings and runways are only found on Green, Sand, Eastern and Tern Islands. 
across many land cover classes. Tables 1.6 and 1.7 provide additional details on land cover categories (also see chap. 2 for further details on Laysan Island land cover).

We did not perform a rigorous quantitative accuracy assessment of our land cover classification due to the logistical constraints of collecting ground-truth data. Our accuracy assessment of land cover classification included visual inspection and comparison with other historical mapping data, field reports, and past botanical surveys (Starr and Martz, 1999b; Starr and Martz, 1999a; Starr and others, 2001; Klavitter, 2006; Starr and others, 2006; Starr and others, 2008). We also solicited expert opinion from field biologists for general classification accuracy (E. Flint, USFWS; P. Hartzell, USFWS [French Frigate Shoals]; T. Speetjens, USFWS [Laysan Island]; M. Stelmach, USFWS [Laysan Island]; and C. Vanderlip, Hawai'i DLNR [Kure Atoll]).

\section{Land Cover Change}

We quantified potential land cover change from SLR using a static vegetation response model. The static vegetation response model predicts that land cover does not shift inland as water levels rise but, rather, that inundated land cover is lost. We overlaid the land cover classification with the inundation grids for each SLR scenario $(+0.5,+1.0,+1.5$, and $+2.0 \mathrm{~m})$ and subtracted the inundation zone from the land cover classification in ArcGIS.

\section{Avifauna Nesting Habitat Classification}

Land cover classes were designated as nesting habitat on the basis of species-specific nesting behavior (see Land Cover Classification section and appendix 3). We calculated speciesspecific potential nesting area as the sum of all utilized land cover classes (table 1.8) without differentiating between vertical habitat structures within land cover classes. For example, tree/ shrub habitat was quantified equally for Great Frigatebird, which nest on top of trees and shrubs, and Red-tailed Tropicbird, which nest on the ground beneath trees and shrubs. We assumed all known nesting habitat types were equally utilized by a given species, ignoring habitat preferences and competition that may vary with habitat availability. Resolution of remotely sensed imagery and seasonal variation in vegetation cover prevented fine-scale nesting habitat classification.

\section{Uncertainty and Model Assumptions}

Scenarios are sequences of postulated events based on specific assumptions. Future SLR is subject to changing environmental conditions, such as carbon dioxide $\left(\mathrm{CO}_{2}\right)$ emissions, changes in temperature, ice sheet dynamics, glacial retreat, and oceanic heat uptake (IPCC, 2007). Therefore, uncertainty in the magnitude and rate of climate change grows with projections further into the future (fig. 1.1). Additionally, passive inundation models have inherent uncertainties. For example, our methods depict physical conditions at a single moment in time, but since

Table 1.7. Land cover classes identified for the low-lying Northwestern Hawaiian Islands from satellite imagery. [Land cover was classified from QuickBird (Kure Atoll [July 2008], Midway Atoll [March 2009], Pearl and Hermes Atoll [October 2007], Lisianski Island [January 2008], French Frigate Shoals [November 2005]) and WorldView-2 (Laysan Island [May 2010]) satellite imagery using IsoCluster methods (ESRI, 2011)]

\begin{tabular}{|c|c|c|c|c|c|c|c|c|c|c|c|c|c|c|c|c|c|c|}
\hline \multirow[b]{2}{*}{ Land cover } & \multirow{2}{*}{ 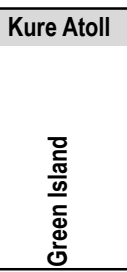 } & \multicolumn{3}{|c|}{ Midway Atoll } & \multicolumn{4}{|c|}{ Pearl and Hermes Atoll } & \multirow[b]{2}{*}{ 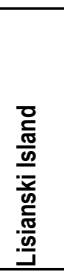 } & \multirow[b]{2}{*}{ 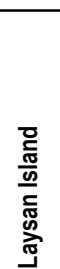 } & \multicolumn{8}{|c|}{ French Frigate Shoals } \\
\hline & & 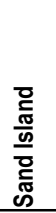 & 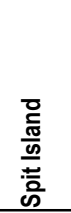 & 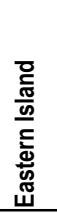 & 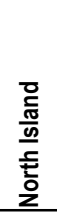 & 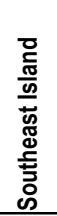 & 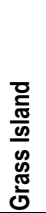 & 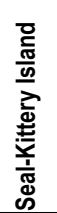 & & & 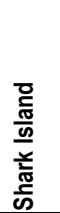 & 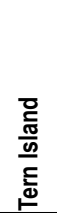 & 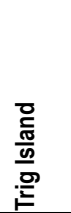 & 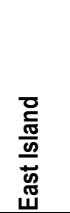 & 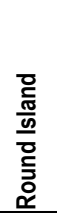 & 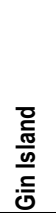 & 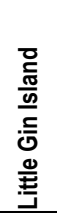 & 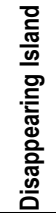 \\
\hline Tree/shrub & $\mathrm{X}$ & $\mathrm{X}$ & $\mathrm{X}$ & $\mathrm{X}$ & & & & & $\bar{X}$ & $\mathrm{X}$ & & $\mathrm{X}$ & & $\mathrm{X}$ & & & & \\
\hline Mixed shrub & & $\mathrm{x}$ & $\mathrm{x}$ & & & & & & $\mathrm{x}$ & $\mathrm{x}$ & & & & & & & & \\
\hline Grass/herbaceous cover & $\mathrm{x}$ & $\mathrm{x}$ & $\mathrm{X}$ & $\mathrm{x}$ & $\mathrm{x}$ & $\mathrm{x}$ & & $\mathrm{x}$ & $\mathrm{x}$ & $\mathrm{x}$ & & $\mathrm{x}$ & & $\mathrm{x}$ & & & & \\
\hline Vine/ground cover & $\mathrm{X}$ & $\mathrm{x}$ & $\mathrm{x}$ & $\mathrm{x}$ & $\mathrm{x}$ & $\mathrm{x}$ & & $\mathrm{x}$ & $\mathrm{X}$ & $\mathrm{x}$ & & & & & & & & \\
\hline Wetland vegetation & & & & & & $\mathrm{X}$ & & & & $\mathrm{X}$ & & & & & & & & \\
\hline Partially vegetated former runway & $\mathrm{X}$ & & & $\mathrm{x}$ & & & & & & & & & & & & & & \\
\hline Bare ground & $\mathrm{x}$ & $\mathrm{X}$ & $\mathrm{X}$ & $\mathrm{x}$ & $\mathrm{X}$ & $\mathrm{X}$ & $\mathrm{X}$ & $\mathrm{X}$ & $\mathrm{X}$ & $\mathrm{X}$ & $\mathrm{x}$ & $\mathrm{X}$ & $\mathrm{X}$ & $\mathrm{X}$ & & $\mathrm{X}$ & $\mathrm{X}$ & \\
\hline Hard pan & & & & & & & & & & $\mathrm{X}$ & & & & & & & & \\
\hline Beach & $\mathrm{x}$ & $\mathrm{x}$ & $\mathrm{x}$ & $\mathrm{x}$ & $\mathrm{x}$ & $\mathrm{x}$ & $\mathrm{x}$ & $\mathrm{x}$ & $\mathrm{x}$ & $\mathrm{x}$ & $\mathrm{x}$ & $\mathrm{x}$ & $\mathrm{x}$ & $\mathrm{x}$ & $\mathrm{x}$ & $\mathrm{x}$ & $\mathrm{X}$ & $\mathrm{x}$ \\
\hline Wetland (unvegetated) & & & & & & & & & & $\mathrm{X}$ & & & & & & & & \\
\hline Wetland (standing water) & $\mathrm{X}$ & $\mathrm{X}$ & $\mathrm{X}$ & $\mathrm{X}$ & & $\mathrm{X}$ & & & & $\mathrm{X}$ & & & & & & & & \\
\hline Human structures & $\mathrm{x}$ & $\mathrm{x}$ & & $\mathrm{x}$ & & & & & & $\mathrm{x}$ & & $\mathrm{x}$ & & & & & & \\
\hline
\end{tabular}


Table 1.8. Known avifauna nesting habitat by land cover class identified from satellite imagery on the low-lying Northwestern Hawaiian Islands. [See appendix 3 for details on nesting behavior]

\begin{tabular}{|c|c|c|c|c|c|c|c|c|c|c|c|c|}
\hline Species & Tree/shrub & $\begin{array}{l}\text { Casuarina } \\
\text { equisetifolia }\end{array}$ & $\begin{array}{c}\text { Pluchea } \\
\text { indica }\end{array}$ & $\begin{array}{l}\text { Tournefortia } \\
\text { argentea }\end{array}$ & Mixed shrub & $\begin{array}{l}\text { Grass/ } \\
\text { herbaceous } \\
\text { cover }\end{array}$ & $\begin{array}{l}\text { Vine/ ground } \\
\text { cover }\end{array}$ & $\begin{array}{l}\text { Wetland } \\
\text { vegetation }\end{array}$ & $\begin{array}{c}\text { Partially } \\
\text { vegetated } \\
\text { former } \\
\text { runway }\end{array}$ & Bare ground & Hard pan $^{a}$ & $\begin{array}{c}\text { Human } \\
\text { structure } \\
\text { (buildings } \\
\text { only) }\end{array}$ \\
\hline Black-footed Albatross & & $\mathrm{X}$ & & & $\mathrm{X}$ & $\mathrm{X}$ & $\mathrm{X}$ & $\mathrm{X}$ & $\mathrm{X}$ & $\mathrm{X}$ & & \\
\hline Laysan Albatross & & $\mathrm{X}$ & & & $\mathrm{X}$ & $\mathrm{X}$ & $\mathrm{X}$ & $\mathrm{X}$ & $\mathrm{X}$ & $\mathrm{X}$ & $\mathrm{X}$ & \\
\hline Short-tailed Albatross & & $\mathrm{X}$ & & & $\mathrm{X}$ & $\mathrm{X}$ & $\mathrm{X}$ & & $\mathrm{X}$ & $\mathrm{X}$ & & \\
\hline Bonin Petrel & $\mathrm{X}$ & $\mathrm{X}$ & $\mathrm{X}$ & $\mathrm{X}$ & $\mathrm{X}$ & $\mathrm{X}$ & $\mathrm{X}$ & & & & & \\
\hline Bulwer's Petrel & & & & & & & & & & $\mathrm{X}$ & & \\
\hline Wedge-tailed Shearwater & $\mathrm{X}$ & $\mathrm{X}$ & $\mathrm{X}$ & $\mathrm{X}$ & $\mathrm{X}$ & $\mathrm{X}$ & $\mathrm{X}$ & & & $\mathrm{X}$ & & $\mathrm{X}^{\mathrm{b}}$ \\
\hline Christmas Shearwater & $\mathrm{X}$ & $\mathrm{X}$ & $\mathrm{X}$ & $\mathrm{X}$ & $\mathrm{X}$ & $\mathrm{X}$ & $\mathrm{X}$ & & $\mathrm{X}$ & & & \\
\hline Tristram's Storm-petrel & $\mathrm{X}$ & $\mathrm{X}$ & $\mathrm{X}$ & $\mathrm{X}$ & $\mathrm{X}$ & $\mathrm{X}$ & $\mathrm{X}$ & & & $\mathrm{X}$ & & \\
\hline Red-tailed Tropicbird & $\mathrm{X}$ & $\mathrm{X}$ & $\mathrm{X}$ & $\mathrm{X}$ & $\mathrm{X}$ & $\mathrm{X}$ & & & $\mathrm{X}$ & & & $X^{b}$ \\
\hline White-tailed Tropicbird & & $\mathrm{X}$ & & & & & & & & & & \\
\hline Masked Booby & & & & & & $\mathrm{X}$ & $\mathrm{X}$ & & $\mathrm{X}$ & $\mathrm{X}$ & $\mathrm{X}$ & \\
\hline Brown Booby & & & & & & $\mathrm{X}$ & $\mathrm{X}$ & & $\mathrm{X}$ & $\mathrm{X}$ & & \\
\hline Red-footed Booby & $\mathrm{X}$ & $\mathrm{X}$ & $\mathrm{X}$ & $\mathrm{X}$ & $\mathrm{X}$ & & & & & & & \\
\hline Great Frigatebird & $\mathrm{X}$ & & $\mathrm{X}$ & $\mathrm{X}$ & $\mathrm{X}$ & & & & & & & \\
\hline Little Tern & & & & & & $X$ & $\mathrm{X}$ & & & $\mathrm{X}$ & & \\
\hline Gray-backed Tern & & & & & & & $\mathrm{X}$ & & $\mathrm{X}$ & $\mathrm{X}$ & $\mathrm{X}$ & \\
\hline Sooty Tern & & & & & & $\mathrm{X}$ & $\mathrm{X}$ & & $\mathrm{X}$ & $\mathrm{X}$ & & \\
\hline Brown Noddy & $\mathrm{X}$ & $\mathrm{X}$ & $\mathrm{X}$ & $\mathrm{X}$ & $\mathrm{X}$ & $\mathrm{X}$ & $\mathrm{X}$ & & $\mathrm{X}$ & $\mathrm{X}$ & & \\
\hline Black Noddy & $\mathrm{X}$ & $\mathrm{X}$ & $\mathrm{X}$ & $\mathrm{X}$ & $X$ & & & & & & & $\mathrm{X}^{\mathrm{b}}$ \\
\hline White Tern & $\mathrm{X}$ & $\mathrm{X}$ & & $\mathrm{X}$ & $\mathrm{X}$ & & & & & & & $\mathrm{X}$ \\
\hline Laysan Teal & $\mathrm{X}$ & $X^{c}$ & $\mathrm{X}$ & $\mathrm{X}$ & $\mathrm{X}$ & $\mathrm{X}$ & $\mathrm{X}$ & & & & & \\
\hline Laysan Finch & $\mathrm{X}$ & & $\mathrm{X}$ & $\mathrm{X}$ & $\mathrm{X}$ & $\mathrm{X}$ & $\mathrm{X}$ & $X^{\mathrm{d}}$ & & & & \\
\hline Nihoa Finch & $\mathrm{X}$ & & $\mathrm{X}$ & $\mathrm{X}$ & $\mathrm{X}$ & $\mathrm{X}$ & $\mathrm{X}$ & & & & & \\
\hline Nihoa Millerbird $^{\mathrm{e}}$ & $\mathrm{X}$ & & $\mathrm{X}$ & $\mathrm{X}$ & $\mathrm{X}$ & $\mathrm{X}$ & $\mathrm{X}$ & & & & & \\
\hline
\end{tabular}

${ }^{a}$ Occurs only on Laysan Island, only species known to nest in habitat type demarked.

bUnder raised buildings at Tern Island, French Frigate Shoals, only.

'Single C. equisetifolia at Laysan Island not classified as potential habitat for Laysan Teal.

dOn Southeast Island, Pearl and Hermes Atoll, only.

${ }^{e}$ Expected habitat use from newly translocated populations to Laysan Island. 
the coastal zone is dynamic, changes in inundation fluctuate with coastal processes, such as changes in sedimentation and erosion (NOAA, 2010b).

DEM accuracy is estimated as RMSE (see chap. 2). Our passive SLR models do not include wave-driven inundation, so the impact of habitat loss and direct effects to resident wildlife during periods of high-wave energy are not considered. In chap. 2 we compare passive inundation models to wave-driven inundation models for a range of SLR scenarios for Laysan Island. We discuss the assumptions and uncertainty of inundation modeling in greater detail in chap. 2 .

In addition to the uncertainties in SLR predictions, the lack of baseline wildlife population data, and the complex interactions among climatic and biotic components of this system influence SLR effects on community and ecosystem dynamics. Most NWHI also lack historical land cover data to analyze change from SLR; however, historical accounts at French Frigate Shoals describe past vegetation communities on now barren islands (for example, Trig and Gin) and completely inundated islands (for example, Whale-Skate; Amerson, 1971; Wetmore 1923 unpub. personal journal reproduced in Olson, 1996). Rising sea levels are expected to cause substantial reductions in beach and shoreline land cover area in other systems (Aiello-Lammens and others, 2011). Webb and Kench (2010) provide evidence of geomorphic change throughout the Pacific including the degree to which islands shift positions on reef platforms due to various factors and processes such as SLR. Evidence from historical observations and aerial photographs of French Frigate Shoals indicated island boundary shifts and inundation (see Hatfield and others, 2012).

Given the model assumptions described above, predictions from SLR models should not be treated as absolute, but should be used as insight to better understand vulnerability and risks to island species, so that climate change mitigation and adaptation strategies can be planned to protect biodiversity. As we learn more about the state of this system under changing climate conditions, models can be improved and updated with new information to reduce uncertainty. In the following section, we report model results from SLR scenarios and downscaled climate projections for individual islands.

\section{Results}

The maximum elevation of the low-lying NWHI ranges from $1.8 \mathrm{~m}$ at Spit (Midway Atoll) and Little Gin Islands (French Frigate Shoals) to $11 \mathrm{~m}$ at Sand Island (Midway Atoll; table 1.9). La Perouse Pinnacle (French Frigate Shoals), a basalt rock, has the highest elevation measured (39.7 m, table 1.9). Thirty-seven percent of the total land area analyzed (excluding La Perouse Pinnacle) was below $2 \mathrm{~m}$; and 87 percent was below $5 \mathrm{~m}$. With passive SLR, just over 4 percent of the total land area was predicted to be inundated at $+1.0 \mathrm{~m}$ and nearly 26 percent at $+2.0 \mathrm{~m}$ (table 1.9). Among the 25 native avian species breeding in the NWHI, we identified two endemic land birds found only on islands with mean elevations less than $4.5 \mathrm{~m}$ (Laysan Teal and Laysan Finch) and four seabird species with global breeding distributions largely limited to the NWHI (Black-footed and Laysan albatross, Bonin Petrel, and Gray-backed Tern; tables 1.10 and 1.11). The State of Hawai' ${ }^{\prime} i$, USFWS, or international conservation committees have listed 11 of the 25 breeding bird species in the NWHI as species of conservation concern or at risk of extinction due to multiple threats (table 1.11).

Across the NWHI, climate change models indicated that increasing temperatures and decreasing precipitation were likely in the future. Overall, annual average maximum temperature was predicted to increase by approximately $2.2^{\circ} \mathrm{C}$ (SD 0.2) across the NWHI by 2100 (table 1.12). The largest increase in annual average maximum temperature of $2.6^{\circ} \mathrm{C}$ (SD 1.7) was predicted for Kure, Midway, and Pearl and Hermes Atolls and Lisianski Island. In the following sections, results of land cover classification, elevation models, SLR, vegetation response, habitat projections, and climate models are presented for each atoll.

\section{Kure Atoll}

\section{Island Information}

Green Island (90.1 hectares, ha) and several small sand spits compose Kure Atoll (fig. 1.4). The small spits do not provide breeding habitat for nesting birds but provide haulout habitat for Hawaiian monk seals and green turtles. Land cover consisted of seven classes: tree/shrub including Scaevola taccada and Tournefortia argentea; grass/herbaceous vegetation including Cenchrus echinatus, Chamaesyce spp., Chenopodium murale, Cynodon dactylon, Eleusine indica, Eragrostis paupera, Eragrostis variabilis, Fimbristylis cymosa, Lobularia maritima, Pseudognaphalium sandwicensium, and Verbesina encelioides; vine/ground cover including Boerhavia repens, Cassytha filiformis, Ipomoea indica, Ipomoea pes-caprae, Sicyos maximowiczii, and Tribulus cistoides; partially vegetated former runway; bare ground; beach; and human structures including buildings (table 1.13; fig. 1.5). Green Island imagery was dominated by grass/herbaceous cover (including Verbesina encelioides, an invasive species of concern), covering 47 percent (42.1 ha) of the island in 2010 (table 1.13). Only 5 percent (4.1 ha) and 21 percent (19.2 ha) of Green Island consisted of bare ground and tree/shrub, respectively (table 1.13).

\section{Sea-Level Rise}

Green Island had a maximum elevation of $7.3 \mathrm{~m}$ and mean of $2.8 \mathrm{~m}$ (SD 2.0; table 1.9, fig. 1.6). More than one-sixth (17 percent) of the total land area was below $2.0 \mathrm{~m}$ elevation and 90 percent was below $4.0 \mathrm{~m}$. The passive inundation model showed a 9 percent loss of total island area at $+1.0 \mathrm{~m}$ SLR and 14 percent at $+2.0 \mathrm{~m}$ SLR (table 1.13, fig. 1.7). Almost no vegetated area was lost at less than $+2.0 \mathrm{~m}$ SLR, at which point only 0.3 percent was lost. Beach area, however, was reduced by 50 percent at $+1.0 \mathrm{~m}$ SLR and 80 percent at $+2.0 \mathrm{~m}$ SLR (table 1.13). Seabird nesting habitat was reduced by less than 8 percent for all seabirds (table 1.14). 
Table 1.9. Mean and maximum elevations of Northwestern Hawaiian Islands, referenced to mean high water (MHW), and derived from digital elevation models (DEMs; 2010, 2011).

Model accuracy is reported as the root-mean-squared error (RMSE) for each island; RMSE calculated from ellipsoidal heights. Land area and percent change were estimated at $+0.5,+1.0$, +1.5 , and +2.0 meters of sea-level rise, assuming passive inundation. Abbreviations: $m$, meters; ha, hectares; \%, percent; SD, standard deviation]

\begin{tabular}{|c|c|c|c|c|c|c|c|c|c|c|c|c|c|c|}
\hline \multirow[b]{2}{*}{ Atoll } & \multirow[b]{2}{*}{ Island } & \multirow[b]{2}{*}{ RMSE (m) } & \multicolumn{3}{|c|}{ DEM Elevation $(\mathrm{m}) 2010$} & \multirow{2}{*}{$\begin{array}{c}0.0 \mathrm{~m} \\
\text { Area (ha) }\end{array}$} & \multicolumn{2}{|c|}{$+0.5 \mathrm{~m}$} & \multicolumn{2}{|c|}{$+1.0 \mathrm{~m}$} & \multicolumn{2}{|c|}{$+1.5 \mathrm{~m}$} & \multicolumn{2}{|c|}{$+2.0 \mathrm{~m}$} \\
\hline & & & Mean & $\pm \mathrm{SD}$ & Max & & Area (ha) & $\%$ change & Area (ha) & $\%$ change & Area (ha) & $\%$ change & Area (ha) & $\%$ change \\
\hline Kure Atoll & Green & 0.11 & 2.8 & \pm 2.0 & 7.3 & 90.1 & 84.6 & -6.1 & 82.4 & -8.6 & 79.8 & -11.4 & 77.3 & -14.2 \\
\hline \multirow[t]{3}{*}{ Midway Atoll } & Sand & 0.05 & 2.5 & \pm 1.2 & 11.0 & 456.8 & 452.2 & -1.0 & 448.7 & -1.8 & 442.2 & -3.2 & 276.8 & -39.4 \\
\hline & Eastern & 0.05 & 2.1 & \pm 0.6 & 7.5 & 133.6 & 131.4 & -1.7 & 128.5 & -3.8 & 122.1 & -8.6 & 81.2 & -39.2 \\
\hline & Spit & 0.05 & 0.8 & \pm 0.4 & 1.8 & 5.3 & 4.6 & -13.2 & 1.4 & -73.6 & 0.0 & -100 & 0.0 & -100 \\
\hline \multirow[t]{6}{*}{ Pearl and Hermes Atoll } & North & 0.12 & 1.4 & \pm 0.5 & 2.5 & 8.4 & 7.5 & -10.7 & 6.4 & -23.8 & 4.4 & -47.6 & 0.9 & -89.3 \\
\hline & Little North & 0.12 & 0.9 & \pm 0.4 & 2.3 & 3.2 & 2.3 & -28.1 & 1.1 & -65.6 & 0.3 & -90.6 & 0.0 & -100 \\
\hline & Southeast & 0.12 & 1.0 & \pm 0.6 & 2.5 & 18.4 & 14.1 & -23.4 & 7.7 & -58.2 & 3.2 & -82.6 & 0.5 & -97.3 \\
\hline & Grass & 0.12 & 1.3 & \pm 0.7 & 2.3 & 3.2 & 2.8 & -13.3 & 2.2 & -31.3 & 1.2 & -62.8 & 0.3 & -90.6 \\
\hline & Seal-Kittery & 0.12 & 1.3 & \pm 0.7 & 2.6 & 13.7 & 11.4 & -16.9 & 9.5 & -30.7 & 5.2 & -62.0 & 2.1 & -84.7 \\
\hline & Lisianski & 0.18 & 3.8 & \pm 1.3 & 7.6 & 147.1 & 145.1 & -1.4 & 143.7 & -2.3 & 142.1 & -3.3 & 140.4 & -4.6 \\
\hline \multicolumn{2}{|c|}{ Laysan Island ${ }^{\mathrm{a}}$ (without groundwater rise) } & 0.09 & 4.3 & \pm 2.3 & 10.7 & 339.4 & 338.3 & -0.3 & 335.8 & -1.1 & 331.2 & -2.4 & 326.6 & -3.8 \\
\hline \multicolumn{2}{|c|}{ Laysan Island $^{\mathrm{a}}$ (with groundwater rise) } & 0.09 & 4.3 & \pm 2.3 & 10.7 & 339.4 & 337.7 & -0.5 & 317.0 & -6.6 & 276.8 & -17.6 & 259.6 & -23.5 \\
\hline \multirow[t]{9}{*}{ French Frigate Shoals } & Shark & 0.05 & 1.2 & \pm 0.3 & 1.9 & 0.3 & 0.3 & 0.0 & 0.2 & -33.3 & 0.0 & -100 & 0.0 & -100 \\
\hline & Tern & 0.05 & 2.3 & \pm 0.5 & 3.4 & 13.8 & 13.7 & -0.7 & 13.2 & -4.3 & 12.5 & -9.4 & 12.0 & -13.0 \\
\hline & Trig $^{\mathrm{b}}$ & 0.15 & 0.5 & \pm 0.8 & 2.5 & 1.4 & 1.3 & -7.1 & 1.1 & -21.4 & 0.8 & -42.9 & 0.3 & -78.6 \\
\hline & East $^{\mathrm{b}}$ & 0.15 & 2.3 & \pm 0.5 & 3.1 & 2.8 & 2.8 & 0.0 & 2.8 & 0.0 & 2.7 & -3.6 & 2.7 & -3.6 \\
\hline & Round $^{\mathrm{b}}$ & 0.15 & 1.0 & \pm 0.6 & 2.0 & 0.1 & 0.1 & 0.0 & 0.0 & -100 & 0.0 & -100 & 0.0 & -100 \\
\hline & La Perouse Pinnacle ${ }^{b}$ & 0.15 & 19.5 & \pm 12.1 & 39.7 & 0.3 & $*$ & $*$ & $*$ & $*$ & $*$ & * & $*$ & $*$ \\
\hline & Gin & 0.05 & 1.6 & \pm 0.5 & 2.9 & 1.7 & 1.4 & -17.6 & 0.9 & -47.1 & 0.3 & -82.4 & 0.0 & -100 \\
\hline & Little Gin ${ }^{\mathrm{b}}$ & 0.15 & 1.0 & \pm 0.4 & 1.8 & 1.6 & 1.5 & -6.2 & 1.1 & -31.3 & 0.2 & -87.5 & 0.0 & -100 \\
\hline & Disappearing & 0.05 & 0.6 & \pm 0.6 & 2.3 & 0.4 & 0.2 & -50.0 & 0.1 & -75.0 & 0.0 & -100 & 0.0 & -100 \\
\hline All atolls and islands ${ }^{\mathrm{c}}$ & & & 1.7 & \pm 1.1 & 11.0 & 1241.3 & 1215.6 & -2.1 & 1186.8 & -4.4 & 1148.2 & -7.5 & 921.1 & -25.8 \\
\hline
\end{tabular}

${ }^{\mathrm{a}}$ Terrestrial area only, lake zone mudflats and open water lake excluded. ${ }^{\mathrm{b}}$ Alternative data source, PhotoSat DEM. ${ }^{\mathrm{c}}$ Total without groundwater rise on Laysan Island; does not include La Perouse

Pinnacle. *Area loss due to SLR not assessed for La Perouse Pinnacle. 
Table 1.10. Global breeding distribution for species of the Northwestern Hawaiian Islands (NWHI), compiled from Fefer and others (1984), Friedlander and others (2009), The Birds of North America Online (A. Poole, editor), and U.S. Fish and Wildlife Service (USFWS, 1998, 2007, 2008a, 2010) species recovery plans.

[Species marked with a bold double $\mathrm{X}$ are expected to be highly vulnerable to habitat loss from sea-level rise due to their limited global breeding distributions largely occurring in the low-lying NWHI (also see table 1.11 and see table 1.1 for sub-species designations)]

\begin{tabular}{|c|c|c|c|c|c|c|}
\hline Species & NWHI & $\begin{array}{c}\text { Main } \\
\text { Hawaiian } \\
\text { Islands }\end{array}$ & $\begin{array}{l}\text { Tropical - Sub- } \\
\text { tropical North } \\
\text { Pacific: Other }\end{array}$ & $\begin{array}{l}\text { Tropical - Sub- } \\
\text { tropical South } \\
\text { Pacific }\end{array}$ & $\begin{array}{l}\text { Tropical to Sub- } \\
\text { tropical Atlantic }\end{array}$ & $\begin{array}{l}\text { Tropical to Sub- } \\
\text { tropical Indian }\end{array}$ \\
\hline Black-footed Albatross & $\mathbf{X X}$ & $\mathrm{X}$ & $\mathrm{X}$ & & & \\
\hline Laysan Albatross & $\mathbf{X X}$ & $\mathrm{X}$ & $\mathrm{X}$ & & & \\
\hline Short-tailed Albatross & $\mathrm{X}$ & & $\mathrm{X}$ & & & \\
\hline Bonin Petrel & $\mathbf{X X}$ & & $\mathrm{X}$ & & & \\
\hline Bulwer's Petrel & $\mathrm{X}$ & $\mathrm{X}$ & $\mathrm{X}$ & $\mathrm{X}$ & $\mathrm{X}$ & $\mathrm{X}$ \\
\hline Wedge-tailed Shearwater & $\mathrm{X}$ & $\mathrm{X}$ & $\mathrm{X}$ & $\mathrm{X}$ & & $\mathrm{X}$ \\
\hline Christmas Shearwater & $\mathrm{X}$ & $\mathrm{X}$ & $\mathrm{X}$ & $\mathrm{X}$ & & \\
\hline Tristram's Storm-petrel & $\mathrm{X}$ & & $\mathrm{X}$ & & & \\
\hline Red-tailed Tropicbird & $\mathrm{X}$ & $\mathrm{X}$ & $\mathrm{X}$ & $\mathrm{X}$ & & $\mathrm{X}$ \\
\hline White-tailed Tropicbird & $\mathrm{X}$ & $\mathrm{X}$ & $\mathrm{X}$ & $\mathrm{X}$ & $\mathrm{X}$ & \\
\hline Masked Booby & $\mathrm{X}$ & & $\mathrm{X}$ & $\mathrm{X}$ & $\mathrm{X}$ & \\
\hline Brown Booby & $\mathrm{X}$ & $\mathrm{X}$ & $\mathrm{X}$ & $\mathrm{X}$ & $\mathrm{X}$ & $\mathrm{X}$ \\
\hline Red-footed Booby & $\mathrm{X}$ & $\mathrm{X}$ & $\mathrm{X}$ & $\mathrm{X}$ & $\mathrm{X}$ & $\mathrm{X}$ \\
\hline Great Frigatebird & $\mathrm{X}$ & $\mathrm{X}$ & $\mathrm{X}$ & $\mathrm{X}$ & $\mathrm{X}$ & $\mathrm{X}$ \\
\hline Little Tern & $\mathrm{X}$ & & $\mathrm{X}$ & $\mathrm{X}$ & $\mathrm{X}$ & $\mathrm{X}$ \\
\hline Gray-backed Tern & $\mathbf{X X}$ & & $\mathrm{X}$ & $\mathrm{X}$ & & \\
\hline Sooty Tern & $\mathrm{X}$ & & $\mathrm{X}$ & $\mathrm{X}$ & $\mathrm{X}$ & $\mathrm{X}$ \\
\hline Brown Noddy & $\mathrm{X}$ & $\mathrm{X}$ & $\mathrm{X}$ & $\mathrm{X}$ & $\mathrm{X}$ & $\mathrm{X}$ \\
\hline Black Noddy & $X$ & $X$ & $X$ & $X$ & $X$ & \\
\hline Blue Noddy & $\mathrm{X}$ & & $\mathrm{X}$ & $\mathrm{X}$ & & \\
\hline White Tern & $\mathrm{X}$ & $\mathrm{X}$ & $\mathrm{X}$ & $\mathrm{X}$ & $\mathrm{X}$ & $\mathrm{X}$ \\
\hline Laysan Teal & $\mathbf{X X}$ & & & & & \\
\hline Laysan Finch & $\mathbf{X X}$ & & & & & \\
\hline Nihoa Finch & $\mathrm{X}$ & & & & & \\
\hline Nihoa Millerbird & $\mathrm{X}$ & & & & & \\
\hline Hawaiian monk seal & $\mathbf{X X}$ & $\mathrm{X}$ & & & & \\
\hline Hawaiian green turtle & $\mathrm{X}$ & $\mathrm{X}$ & & & & \\
\hline Amaranthus brownii & $\mathrm{X}$ & & & & & \\
\hline Cyperus pennatiformis var. bryanii & $\mathbf{X X}$ & & & & & \\
\hline Pritchardia remota & $\mathrm{X}$ & & & & & \\
\hline Schiedea verticillata & $\mathrm{X}$ & & & & & \\
\hline Sesbania tomentosa & $\mathrm{X}$ & $\mathrm{X}$ & & & & \\
\hline
\end{tabular}


Table 1.11. Conservation status assessments and population counts of species breeding (propagating) in the Northwestern Hawaiian Islands (NWHI).

List compiled from the International Union for Conservation of Nature (IUCN; http://www.iucnredlist.org), Nature Serve (http://www.natureserve.org), the USFWS Endangered Species Program (USFWS; http://fws.gov/endangered/) and its list of Birds of Conservation Concern (BCC; USFWS, 2008b), and the State of Hawaii' (Department of Land and Natural Resources, DLNR $\mathrm{http} / / /$ hawaii.gov/dlnr/dofaw). Unless otherwise cited, population counts are not validated, but they are assumed to be indicators of breeder abundance; however, many island counts are more than 28 years old. These population counts were compiled from a variety of sources, including the most recent survey of all NWHI conducted in 1978-1982 (Fefer and others, 1984), and contain updates to abundance indices when available (for details, see Pyle and Pyle, 2009). The sampling error, detection probability, and confidence interval of reported population values are unknown, and population values for many islands have not been updated in 28 years. Abbreviations: USFWS, U.S. Fish and Wildlife Service; USGS, U.S. Geological Survey]

\begin{tabular}{|c|c|c|c|c|c|c|}
\hline \multirow[b]{2}{*}{ Common name } & \multicolumn{4}{|c|}{ Status } & \multicolumn{2}{|r|}{ Population } \\
\hline & IUCN & Nature Serve & U.S. Federal & State of Hawai i & $\begin{array}{l}\text { NWHI breeding } \\
\text { pairs reported }\end{array}$ & $\begin{array}{l}\text { Proportion of global breeding } \\
\text { population restricted to NWHI }\end{array}$ \\
\hline$\overline{\text { Black-footed Albatross }}$ & Vulnerable & G3 - Vulnerable & $\overline{\mathrm{BCC}}$ & Threatened & $64,000(2011)^{\mathrm{a}}$ & $>0.95^{\mathrm{a}}$ \\
\hline Laysan Albatross & Near Threatened & G3 - Vulnerable & $\mathrm{BCC}$ & & $590,179(2011)^{\mathrm{a}}$ & $>0.95^{\mathrm{a}}$ \\
\hline Short-tailed Albatross & Vulnerable & G1 - Critically Imperiled & Endangered & Endangered & $2(2012)^{b, c}$ & $<0.05^{\mathrm{g}}$ \\
\hline Bonin Petrel & Least Concern & Not evaluated & & & 396,150 & $0.5-0.95^{\mathrm{g}}$ \\
\hline Bulwer's Petrel & Least Concern & G4 - Apparently Secure & & & 92,370 & $0.05-0.5^{\mathrm{g}}$ \\
\hline Wedge-tailed Shearwater & Least Concern & G4 - Apparently Secure & & & 228,800 & $0.05-0.5^{\mathrm{h}}$ \\
\hline Christmas Shearwater & Least Concern & G3 - Vulnerable & $\mathrm{BCC}$ & & 2,815 & $0.05-0.5^{\mathrm{h}}$ \\
\hline Tristram's Storm-petrel & Near Threatened & G3 - Vulnerable & $\mathrm{BCC}$ & & 6,030 & $0.05-0.5^{\mathrm{h}}$ \\
\hline Red-tailed Tropicbird & Least Concern & G4 - Apparently Secure & & & 12,800 & $0.05-0.5^{\mathrm{h}}$ \\
\hline White-tailed Tropicbird & Least Concern & G5 - Secure & & & 5 & $<0.05^{\mathrm{h}}$ \\
\hline Masked Booby & Least Concern & G5 - Secure & & & 2,215 & Data needed \\
\hline Brown Booby & Least Concern & G5 - Secure & & & 425 & $<0.05^{\mathrm{h}}$ \\
\hline Red-footed Booby & Least Concern & G5 - Secure & & & 7,450 & $0.05-0.5^{\mathrm{h}}$ \\
\hline Great Frigatebird & Least Concern & G4 - Apparently Secure & & & 10,345 & $0.05-0.5^{\mathrm{h}}$ \\
\hline Little Tern & Least Concern & Not evaluated & & & $20^{\mathrm{d}}$ & $<0.05^{\mathrm{g}}$ \\
\hline Gray-backed Tern & Least Concern & G3 - Vulnerable & & & 43,225 & $0.5-0.95^{\mathrm{h}}$ \\
\hline Sooty Tern & Least Concern & G5 - Secure & & & $1,190,400$ & $0.05-0.5^{\mathrm{h}}$ \\
\hline Blue Noddy & Least Concern & G4 - Apparently Secure & & & 3,780 & Data needed \\
\hline Brown Noddy & Least Concern & G5 - Secure & & & 76,250 & $0.05-0.5^{\mathrm{h}}$ \\
\hline Black Noddy & Least Concern & G5 - Secure & & & 15,050 & $0.05-0.5^{\mathrm{h}}$ \\
\hline White Tern & Least Concern & G4 - Apparently Secure & & Threatened & 25,215 & $0.05-0.5^{\mathrm{g}}$ \\
\hline Laysan Teal & Critically Endangered & G1 - Critically Imperiled & Endangered & Endangered & $500-800(2011)^{\mathrm{e}}$ & 1 \\
\hline Laysan Finch & Vulnerable & G1 - Critically Imperiled & Endangered & Endangered & $5,000-20,000^{f}$ & 1 \\
\hline Nihoa Finch & Critically Endangered & G1 - Critically Imperiled & Endangered & Endangered & $2,100-3,550^{\mathrm{f}}$ & 1 \\
\hline Nihoa Millerbird & Critically Endangered & G1 - Critically Imperiled & Endangered & Endangered & $250-999^{f}$ & 1 \\
\hline Hawaiian monk seal & Critically Endangered & G2 - Imperiled & Endangered & Endangered & $* 1,200-1,300^{\mathrm{i}}$ & $0.5-0.95^{\mathrm{i}}$ \\
\hline Hawaiian green turtle & Endangered & G3 - Vulnerable & Endangered & Endangered & $* 61,000^{\mathrm{j}}$ & $0.5-0.95^{\mathrm{j}}$ \\
\hline Amaranthus brownii & Critically Endangered & G1 - Critically Imperiled & Endangered & Endangered & $*>40^{\mathrm{k}}$ & 1 \\
\hline Cyperus pennatiformis var. bryanii & & G1 - Critically Imperiled & Endangered & Endangered & $* 488^{1}$ & 1 \\
\hline Pritchardia remota & Endangered & G1 - Critically Imperiled & Endangered & Endangered & $* 680^{\mathrm{m}}$ & 1 \\
\hline Schiedea verticillata & & G1 - Critically Imperiled & Endangered & Endangered & $* 359^{\mathrm{m}}$ & 1 \\
\hline Sesbania tomentosa & & G2 - Imperiled & Endangered & Endangered & $* 1,600-2,000^{\mathrm{n}}$ & Data needed \\
\hline
\end{tabular}

*Denotes individuals, not breeding pairs. ${ }^{a}$ Flint (2011). ${ }^{b}$ USFWS unpub. data. ${ }^{c} \mathrm{C}$. Vanderlip, Hawai' i DLNR, written commun. ${ }^{\mathrm{d} F r i e d l a n d e r}$ and others (2009). ${ }^{\mathrm{e}}$ Total number of mature individuals, USFWS/USGS, unpublished data. ${ }^{\mathrm{f}}$ Total number of mature individuals, from Birdlife International (www.Birdlife.org). ${ }^{\mathrm{g}}$ Calculated from values of NWHI breeding pairs and global

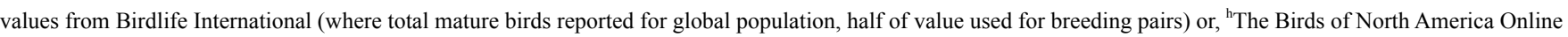
(http://bna.birds.cornell.edu/bna). ${ }^{\mathrm{i}}$ Antonelis and others (2006). ${ }^{\mathrm{j} B a l a z s}$ and Chaloupka (2004). ${ }^{\mathrm{k} U S F W S}$ (2007). ${ }^{\mathrm{l} U S F W S}$ (2008a). ${ }^{\mathrm{m} U S F W S}$ (1998). ${ }^{\mathrm{n} U S F W S}$ (2010). 
Table 1.12. Predicted (2100) annual average maximum temperature and total precipitation from interpolated climate surfaces. [Interpolation conducted with WorldClim data (Hijmans and others, 2005) at a central location at each atoll or island. Predictions from six general circulation models are presented (Intergovernmental Panel on Climate Change (IPCC) 2007). Abbreviations: SD, standard deviation; ${ }^{\circ} \mathrm{C}$, degrees Celsius; mm, millimeters; see table 1.4 for model name descriptions]

\begin{tabular}{|c|c|c|c|c|c|c|c|c|c|c|c|c|c|c|}
\hline & \multicolumn{2}{|c|}{$\begin{array}{l}\text { WorldClim } \\
(1960-1990)\end{array}$} & \multicolumn{12}{|c|}{ Predicted Change (2100) } \\
\hline & Annual & $\pm \mathrm{SD}$ & CGCM3.1.T63 & $\pm S D$ & GFDL CM2.0 & $\pm \mathrm{SD}$ & GFDL CM2.1 & $\pm S D$ & ECHAM5 & $\pm S D$ & CGCM2.3.2A & $\pm S D$ & HADCM3 & $\pm S D$ \\
\hline \multicolumn{15}{|c|}{ Average maximum temperature $\left({ }^{\circ} \mathrm{C}\right)$} \\
\hline Kure Atoll & 23.4 & 0.0 & 1.8 & 1.2 & 2.2 & 1.4 & 2.5 & 1.6 & 2.3 & 1.5 & 2.6 & 1.7 & 1.9 & 1.2 \\
\hline Midway Atoll & 23.6 & 0.0 & 1.8 & 1.2 & 2.1 & 1.4 & 2.5 & 1.7 & 2.3 & 1.5 & 2.6 & 1.7 & 1.9 & 1.2 \\
\hline Pearl and Hermes Atoll & 23.5 & 0.0 & 1.8 & 1.2 & 2.1 & 1.4 & 2.6 & 1.7 & 2.3 & 1.5 & 2.6 & 1.7 & 1.9 & 1.3 \\
\hline Lisianski Island & 23.5 & 0.0 & 1.8 & 1.2 & 2.1 & 1.4 & 2.6 & 1.7 & 2.3 & 1.5 & 2.6 & 1.7 & 2.0 & 1.3 \\
\hline French Frigate Shoals & 25.3 & 0.1 & 2.0 & 1.3 & 1.9 & 1.2 & 2.4 & 1.5 & 2.3 & 1.5 & 2.2 & 1.4 & 2.2 & 1.4 \\
\hline \multicolumn{15}{|l|}{ Total precipitation (mm) } \\
\hline Kure Atoll & 818.7 & 1.3 & -85.6 & 55.7 & -99.8 & 65.0 & -84.8 & 55.3 & -56.0 & 36.5 & -27.9 & 18.2 & -103.5 & 67.4 \\
\hline Midway Atoll & 801.6 & 1.3 & -89.3 & 58.2 & -107.0 & 69.7 & -87.6 & 57.1 & -64.6 & 42.1 & -28.9 & 18.9 & -87.0 & 56.7 \\
\hline Pearl and Hermes Atoll & 812.1 & 1.5 & -90.3 & 58.8 & -121.3 & 79.0 & -91.6 & 59.6 & -91.5 & 59.6 & -44.0 & 28.7 & -67.7 & 44.1 \\
\hline Lisianski Island & 846.6 & 1.7 & -91.9 & 59.9 & -138.7 & 90.3 & -95.5 & 62.2 & -117.2 & 76.3 & -51.4 & 33.5 & -54.2 & 35.3 \\
\hline
\end{tabular}

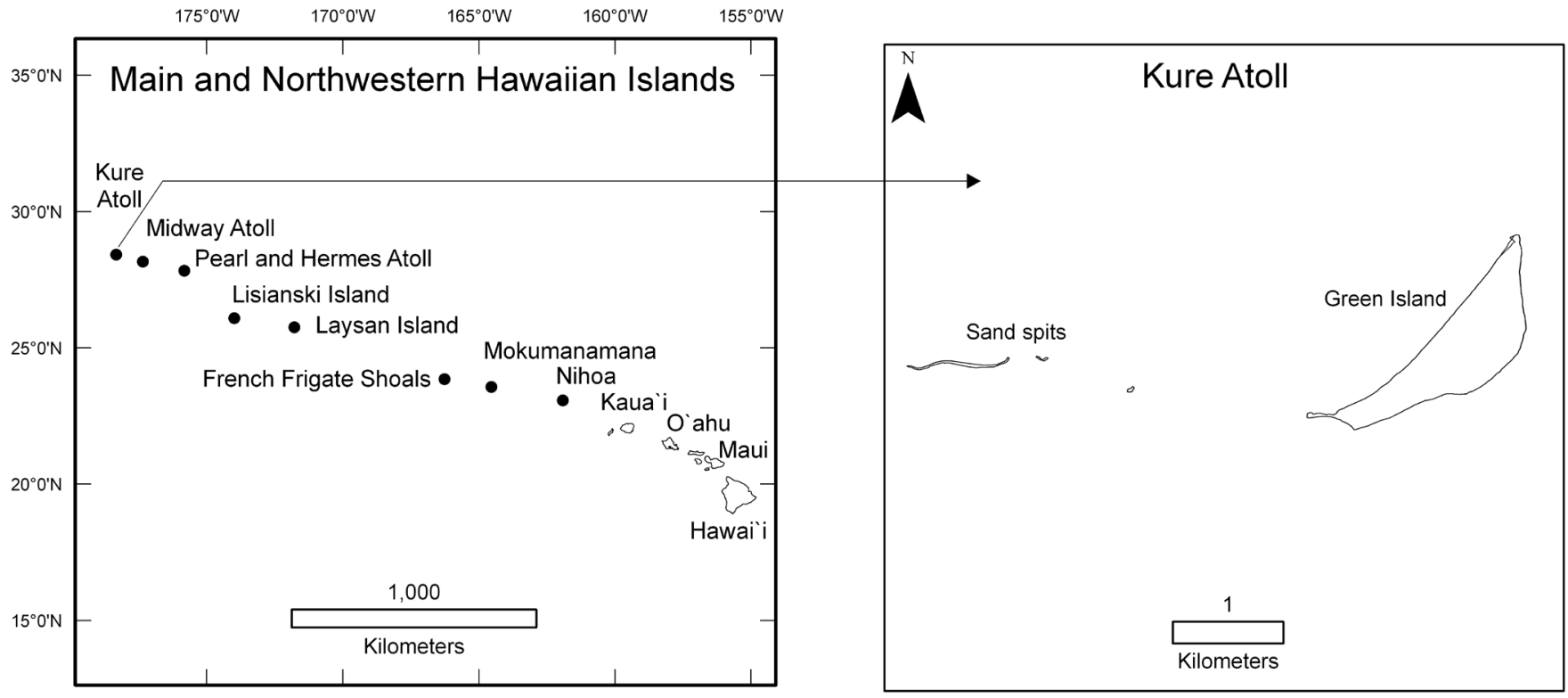

Figure 1.4. Map showing the location of Kure Atoll, the northernmost coral atoll in the world. Kure Atoll consists of Green Island and several small sand spits. 


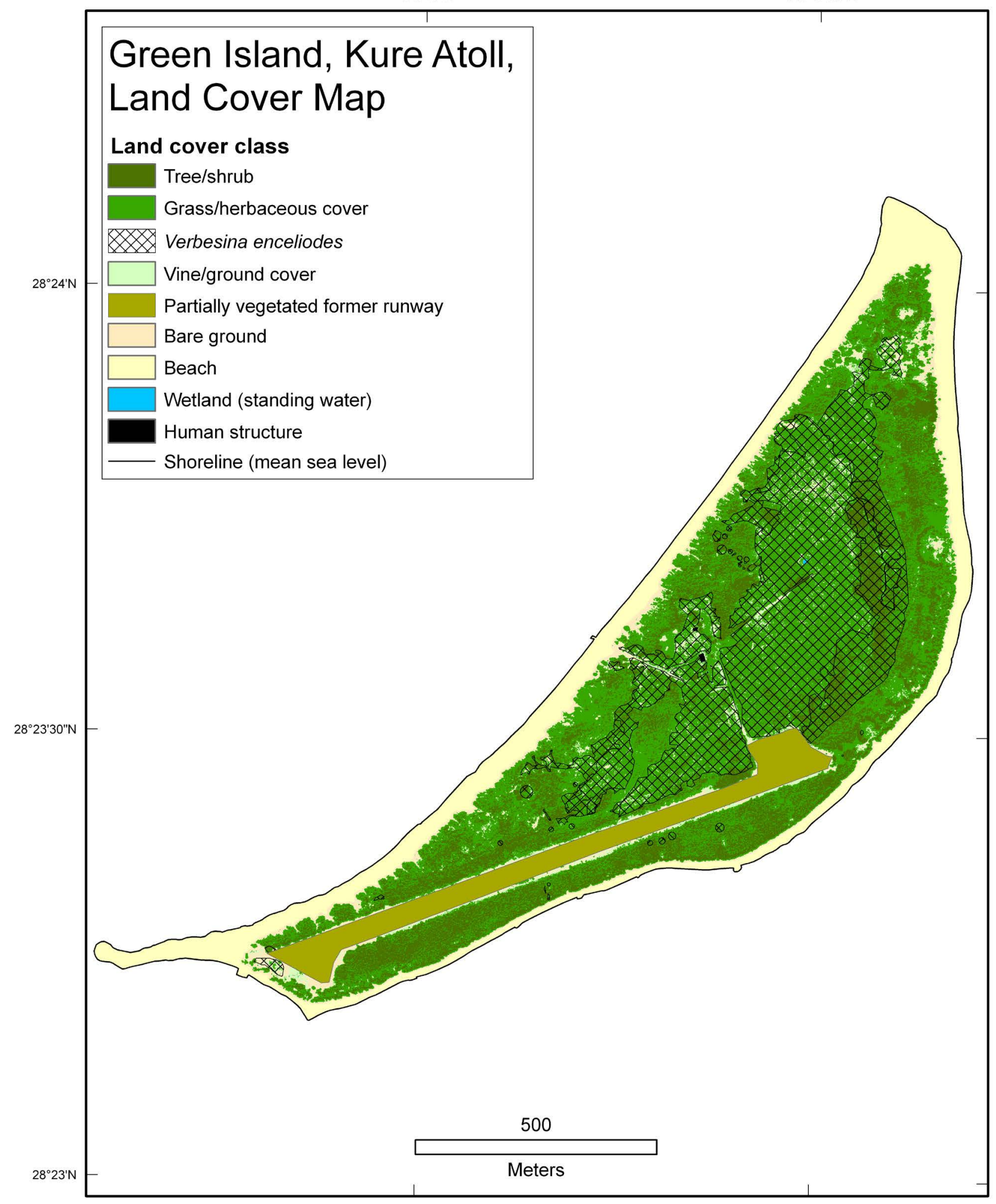

Figure 1.5. Green Island, Kure Atoll, land cover classification map developed using primarily unsupervised classification methods from a Digital Globe QuickBird satellite image (July 2008). Verbesina encelioides distribution mapped from 2007 field data (Vanderlip and others, 2007). Active management to remove $V$. encelioides after 2007 has substantially altered the distribution and abundance of this invasive species. Additional details on land cover and species composition are given in table 1.13. 


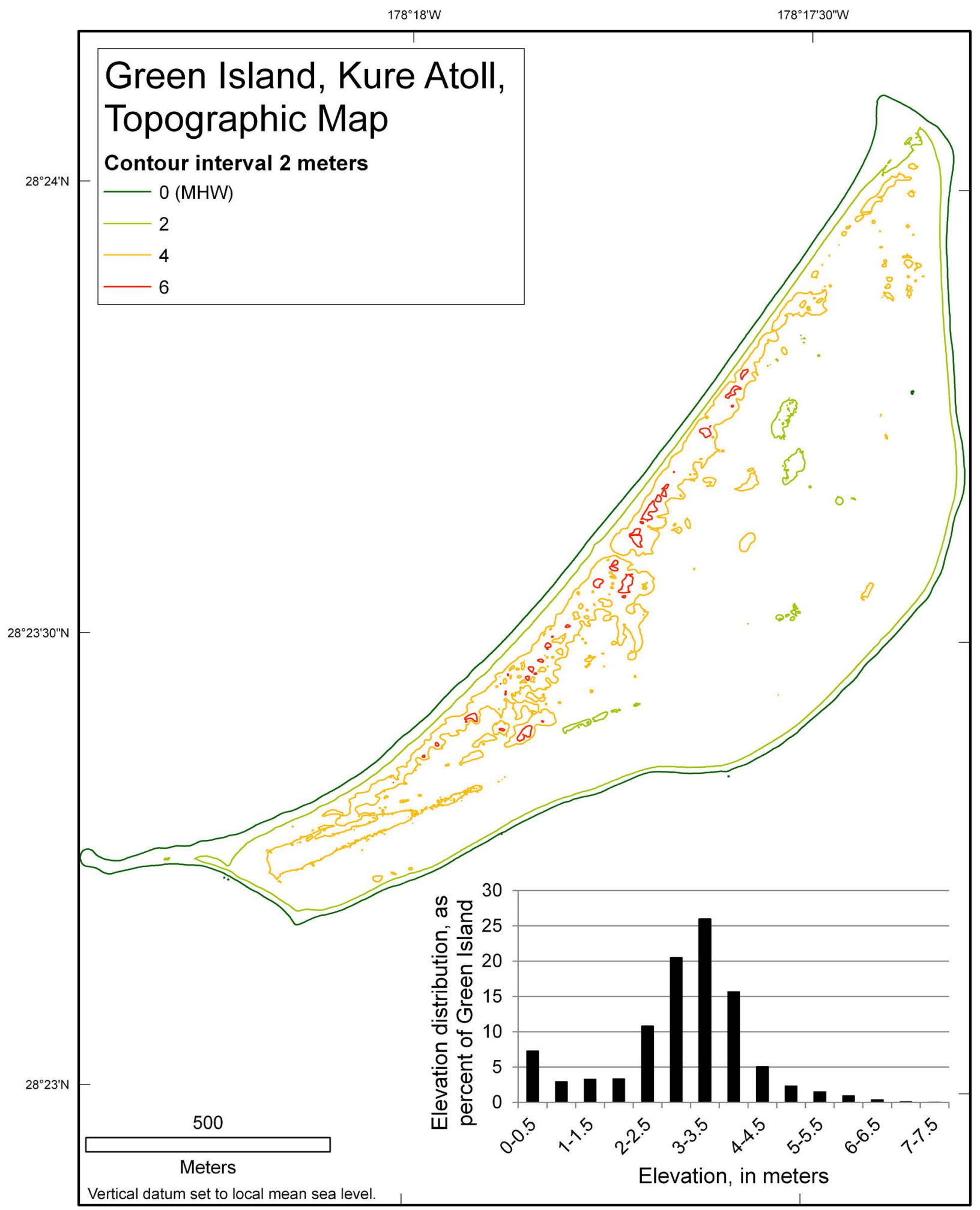

Figure 1.6. Topographic map of Green Island, Kure Atoll, showing mean high water (MHW) and 2-meter $(\mathrm{m})$ contour lines with a graph of the percentage of land in 0.5-m elevation bins. Elevations determined from U.S. Geological Survey lidar data (2010) with a vertical root-mean-squared error of $0.11 \mathrm{~m}$. 


\section{Green Island, Kure Atoll, Passive Inundation Scenarios}

Inundation area

\section{Elevation (m)}
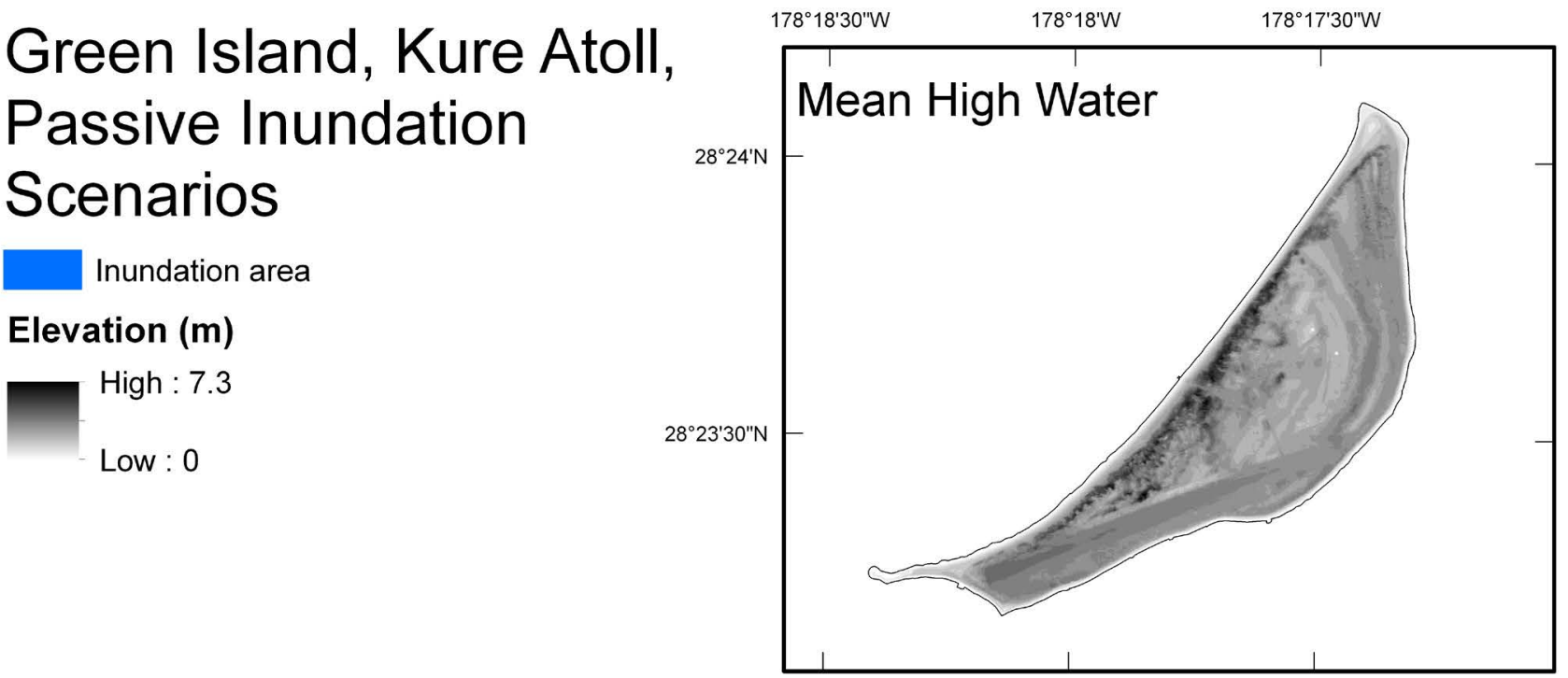

\section{$+0.5 \mathrm{~m} \mathrm{SLR}$}
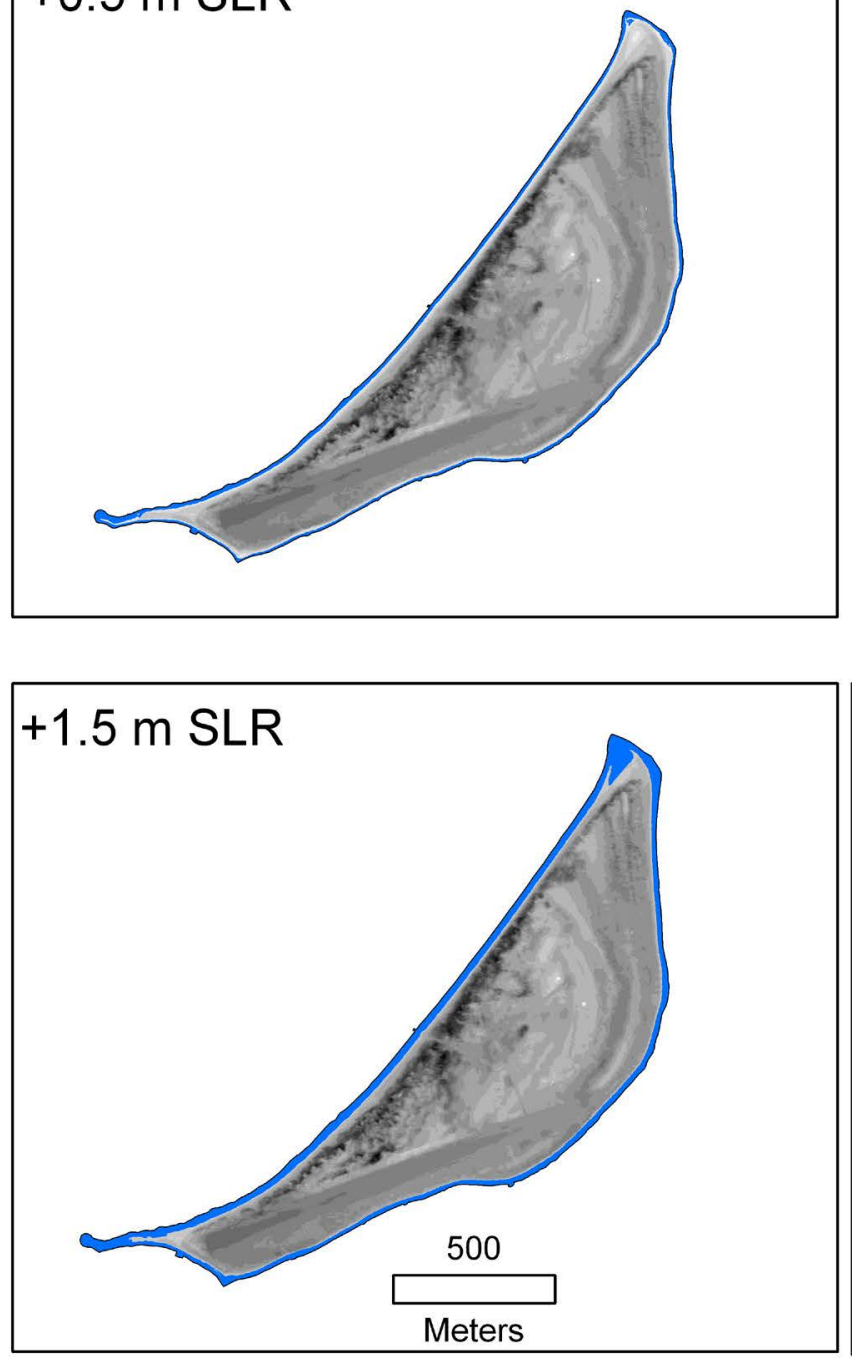

\section{$+1.0 \mathrm{~m}$ SLR}

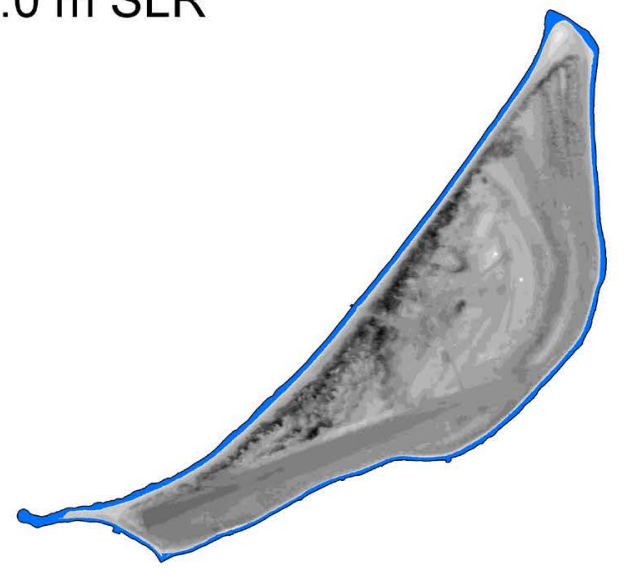

\section{$+2.0 \mathrm{~m}$ SLR}

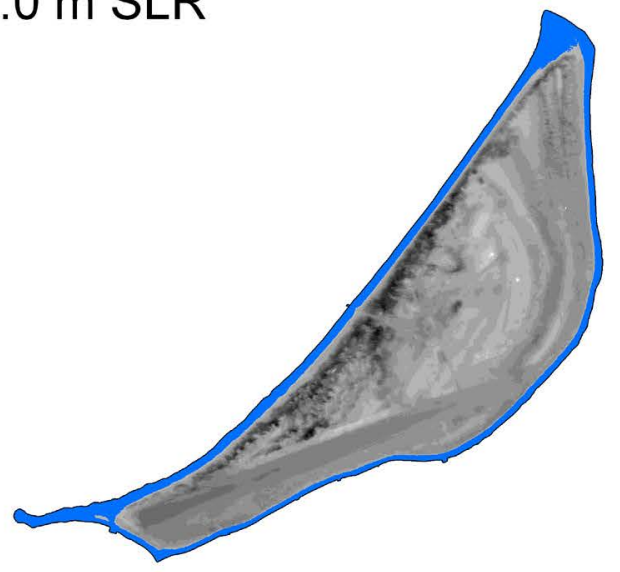

Figure 1.7. Green Island, Kure Atoll, passive inundation scenario maps for five sea levels: mean high water through $+2.0 \mathrm{~m}$ (meters) sea-level rise (SLR) at 0.5-m increments. Inundation scenarios are based on U.S. Geological Survey lidar-derived elevations with a root-mean-squared error of $0.11 \mathrm{~m}$. 
Table 1.13. Total land area and percent change in area for each of six land cover types at Green Island, Kure Atoll, with four sea-level rise (SLR) scenarios $(+0.5,+1.0,+1.5$, and +2.0 meters).

[Areas calculated from Digital Globe QuickBird satellite imagery (July 2008). Abbreviations: m, meters; ha, hectares; \%, percent]

\begin{tabular}{|c|c|c|c|c|c|c|c|c|c|}
\hline \multirow[b]{3}{*}{ Land cover } & \multirow{3}{*}{$\begin{array}{c}0.0 \mathrm{~m} \\
\text { Area (ha) }\end{array}$} & \multicolumn{8}{|c|}{ Change from SLR } \\
\hline & & \multicolumn{2}{|c|}{$+0.5 \mathrm{~m}$} & \multicolumn{2}{|c|}{$+1.0 \mathrm{~m}$} & \multicolumn{2}{|c|}{$+1.5 \mathrm{~m}$} & \multicolumn{2}{|c|}{$+2.0 \mathrm{~m}$} \\
\hline & & Area (ha) & $\%$ change & Area (ha) & $\%$ change & Area (ha) & $\%$ change & Area (ha) & $\%$ change \\
\hline Tree/shrub & 19.2 & 19.2 & 0.0 & 19.2 & 0.0 & 19.2 & 0.0 & 19.1 & -0.5 \\
\hline Vine/ground cover & 3.3 & 3.3 & 0.0 & 3.3 & 0.0 & 3.3 & 0.0 & 3.3 & 0.0 \\
\hline Partially vegetated former runway & 6.0 & 6.0 & 0.0 & 6.0 & 0.0 & 6.0 & 0.0 & 6.0 & 0.0 \\
\hline Beach & 15.4 & 9.9 & -35.7 & 7.7 & -50.0 & 5.3 & -65.6 & 3.1 & -79.9 \\
\hline Total island size & 90.1 & 84.6 & -6.1 & 82.3 & -8.6 & 79.8 & -11.4 & 77.3 & -14.2 \\
\hline
\end{tabular}

Table 1.14. Total potential nesting habitat area and percent change in nesting habitat area for breeding avifauna at Green Island, Kure Atoll, with four sea-level rise (SLR) scenarios $(+0.5,+1.0$, +1.5 , and +2.0 meters).

[Areas calculated from land cover classes with Digital Globe QuickBird satellite imagery (July 2008). We assumed percent change based on uniform density across nesting habitat for each species. Abbreviations: $\mathrm{m}$, meters; ha, hectares]

\begin{tabular}{|c|c|c|c|c|c|}
\hline \multirow[b]{2}{*}{ Species } & \multirow{2}{*}{$\begin{array}{c}\text { Area (ha) } \\
0.0 \mathrm{~m}\end{array}$} & \multicolumn{4}{|c|}{ Percent Change from SLR } \\
\hline & & $+0.5 \mathrm{~m}$ & $+1.0 \mathrm{~m}$ & $+1.5 \mathrm{~m}$ & $+2.0 \mathrm{~m}$ \\
\hline Black-footed Albatross & 55.5 & 0.0 & -0.2 & -0.4 & -0.7 \\
\hline Laysan Albatross & 55.5 & 0.0 & -0.2 & -0.4 & -0.7 \\
\hline Short-tailed Albatross & 55.5 & 0.0 & -0.2 & -0.4 & -0.7 \\
\hline Bonin Petrel & 64.6 & 0.0 & 0.0 & -0.2 & -0.3 \\
\hline Bulwer's Petrel & 4.1 & 0.0 & -2.4 & -2.4 & -7.3 \\
\hline Wedge-tailed Shearwater & 68.7 & 0.0 & -0.1 & -0.3 & -0.7 \\
\hline Christmas Shearwater & 70.6 & 0.0 & 0.0 & -0.1 & -0.3 \\
\hline Tristram's Storm-petrel & 68.7 & 0.0 & -0.1 & -0.3 & -0.7 \\
\hline Red-tailed Tropicbird & 67.3 & 0.0 & 0.0 & -0.1 & -0.3 \\
\hline Masked Booby & 55.5 & 0.0 & -0.2 & -0.4 & -0.7 \\
\hline Brown Booby & 55.5 & 0.0 & -0.2 & -0.4 & -0.7 \\
\hline Red-footed Booby & 19.2 & 0.0 & 0.0 & 0.0 & -0.5 \\
\hline Great Frigatebird & 19.2 & 0.0 & 0.0 & 0.0 & -0.5 \\
\hline Gray-backed Tern & 13.4 & 0.0 & -0.7 & -0.7 & -2.2 \\
\hline Sooty Tern & 55.5 & 0.0 & -0.2 & -0.4 & -0.7 \\
\hline Brown Noddy & 74.7 & 0.0 & -0.1 & -0.3 & -0.7 \\
\hline Black Noddy & 19.2 & 0.0 & 0.0 & 0.0 & -0.5 \\
\hline White Tern & 19.2 & 0.0 & 0.0 & 0.0 & -0.5 \\
\hline
\end{tabular}




\section{Midway Atoll}

\section{Island Information}

Sand, Spit, and Eastern Islands compose Midway Atoll (fig. 1.8). Sand Island was 456.8 ha, Spit Island was 5.3 ha, and Eastern Island was 133.6 ha. Land cover consisted of nine general classes: tree/shrub including Casuarina equisetifolia, Coccoloba uvifera, Hibiscus tiliaceus, Lepidium bidentatum, Scaevola taccada, Terminalia catappa, Tournefortia argentea; mixed shrub; grass/herbaceous cover including Cenchrus echinatus, Eragrostis paupera, Eragrostis variabilis, Euphorbia spp., Fimbristylis cymosa, Lepturus repens, Lobularia maritime, Pseudognaphalium sandwicensium, Psilotum nudum, Solanum nelsonii, Verbesina encelioides; vine/ground cover including Bidens alba, Boerhavia repens, Cassytha filiformis, Ipomoea indica, Ipomoea pes-caprae, Portulaca lutea, and Tribulus cistoides; partially vegetated former runway; bare ground; beach; wetland (standing water); and human structures including buildings, roads, and runways (tables 1.15-17; figs. 1.9-11). Two species-specific land cover classes based on historical distribution data included: Casuarina equisetifolia and Tournefortia argentea (table 1.15). At Sand Island the dominant land cover categories were grass/herbaceous cover (133.8 ha) and human structures including tarmac (129.3 ha; table 1.15). Spit Island land cover consisted mostly of bare ground (2.3 ha), grass/herbaceous cover (1.1 ha), mixed shrub (0.8 ha), and beach ( $0.8 \mathrm{ha}$; table 1.16$)$. Grass/herbaceous cover (74.4 ha) and partially vegetated runway (31.6 ha) predominated at Eastern Island (table 1.17).

\section{Sea-Level Rise}

\section{Sand Island}

The maximum elevation of Sand Island was $11.0 \mathrm{~m}$ with a mean of $2.5 \mathrm{~m}$ (SD 1.2; table 1.9). Of the total land area, 42 percent of Sand Island was below $2.0 \mathrm{~m}$ elevation and 90 percent below $4.0 \mathrm{~m}$ (fig. 1.12). With the passive inundation model, 2 percent of total area was lost at $+1.0 \mathrm{~m}$ SLR and 39 percent at $+2.0 \mathrm{~m}$ SLR (table 1.15, fig. 1.13). Loss of vegetated area was minimal (less than 1 percent) at less than +2.0 $\mathrm{m}$ SLR, at which point 33 percent of total vegetated area was lost. Beach area, however, was reduced by 31 percent at +1.0 $\mathrm{m} \mathrm{SLR}$ and 71 percent at $+2.0 \mathrm{~m}$ SLR (table 1.15). Less than 2 percent of potential nesting habitat for bird species was lost at $+1.5 \mathrm{~m}$ SLR; however, at $+2.0 \mathrm{~m}$ SLR $32-38$ percent was lost (table 1.18).

\section{Spit Island}

The maximum elevation of Spit Island was $1.8 \mathrm{~m}$ with a mean of $0.8 \mathrm{~m}$ (SD 0.4; table 1.9). Of all the islands of Midway Atoll, proportional land area loss was greatest at Spit Island with 74 percent lost at $+1.0 \mathrm{~m}$ SLR and complete inundation at $+1.5 \mathrm{~m}$ SLR (table 1.16, figs. 1.14-15). Vegetated area losses were 62 percent at $+1.0 \mathrm{~m}$ SLR and 100 percent at $+1.5 \mathrm{~m}$ SLR (table 1.16). Beach and wetland were completely inundated at $+1.0 \mathrm{~m}$ SLR (table 1.16), and all nesting habitat was lost at $+1.5 \mathrm{~m}$ SLR (table 1.19).

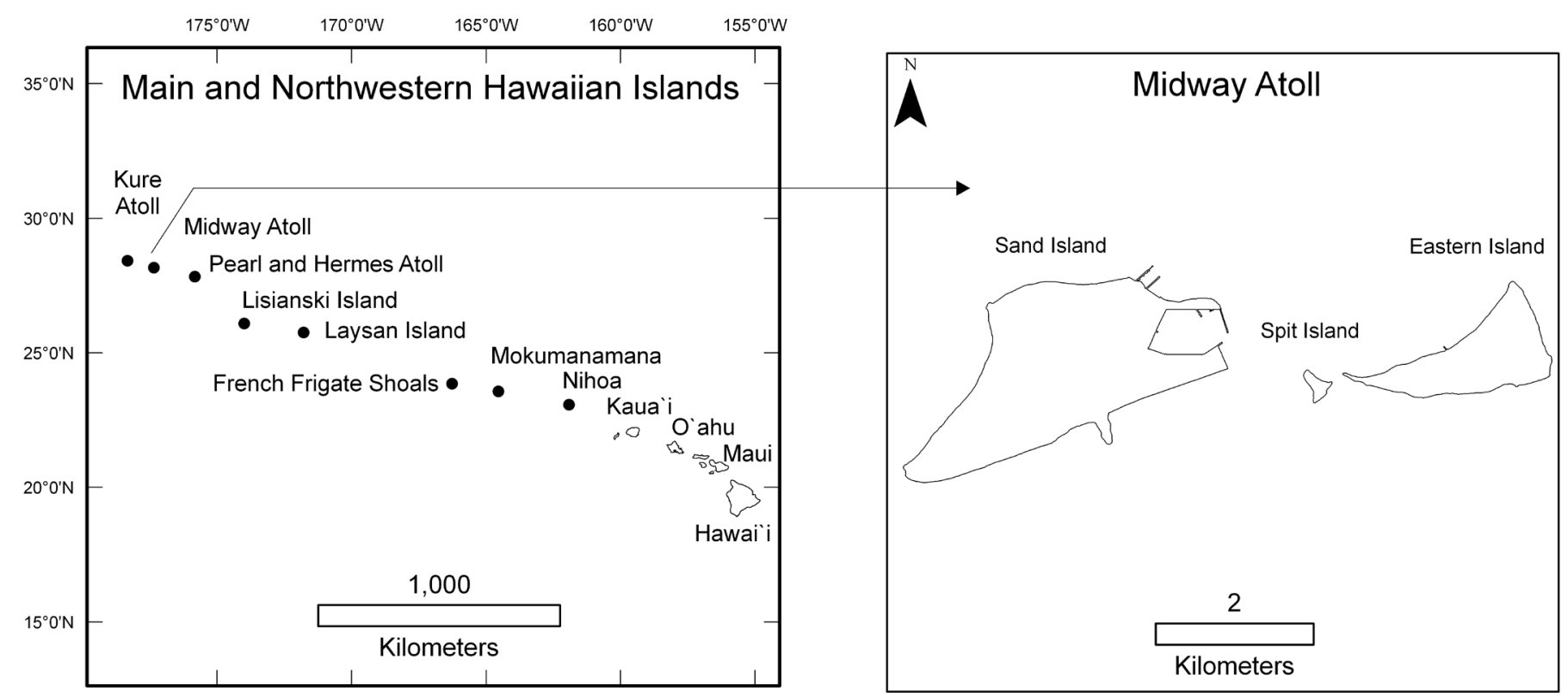

Figure 1.8. Maps showing the location of Midway Atoll, a coral atoll located between Kure Atoll to the northwest and Pearl and Hermes Atoll to the southeast. Sand, Spit, and Eastern Islands are the only permanent land masses at Midway Atoll. 


\section{Eastern Island}

The maximum elevation of Eastern Island was $7.5 \mathrm{~m}$ with a mean of $2.1 \mathrm{~m}$ (SD 0.6; table 1.9). Of the total land area of Eastern Island, 42 percent was below 2.0 m elevation and 99 percent was below $3.5 \mathrm{~m}$ (fig. 1.16). With the passive inundation model, 4 percent of total area was lost at $+1.0 \mathrm{~m}$ SLR and 39 percent at $+2.0 \mathrm{~m}$ SLR (table 1.17, fig. 1.17). Total beach area was reduced by 45 percent and bare ground by 15 percent at $+1.0 \mathrm{~m} \mathrm{SLR}$; at +2.0 $\mathrm{m} \mathrm{SLR}$ all land cover classes were reduced by more than one-quarter (table 1.17). Habitat losses ranged from 2 to 5 percent for all bird species at $+1.5 \mathrm{~m}$ SLR (table 1.20). However, at $+2.0 \mathrm{~m}$ SLR breeding habitat for all species was expected to be reduced by 29-37 percent (table 1.20, fig. 1.17).

Table 1.15. Total land area and percent change in area for each of ten land cover types at Sand Island, Midway Atoll, with four sea-level rise $(S L R)$ scenarios $(+0.5,+1.0,+1.5$, and +2.0 meters).

[Areas calculated from Digital Globe QuickBird satellite imagery (March 2009) and historical field data (Laniawe, 2004). The land cover class "partially vegetated former runway" was not classified at Sand Island. Abbreviations: $m$, meters; ha, hectares; \%, percent]

\begin{tabular}{|c|c|c|c|c|c|c|c|c|c|}
\hline \multirow[b]{3}{*}{ Land cover } & \multirow{3}{*}{$\begin{array}{c}0.0 \mathrm{~m} \\
\text { Area (ha) }\end{array}$} & \multicolumn{8}{|c|}{ Change from SLR } \\
\hline & & \multicolumn{2}{|c|}{$+0.5 \mathrm{~m}$} & \multicolumn{2}{|c|}{$+1.0 \mathrm{~m}$} & \multicolumn{2}{|c|}{$+1.5 \mathrm{~m}$} & \multicolumn{2}{|c|}{$+2.0 \mathrm{~m}$} \\
\hline & & Area (ha) & $\%$ change & Area (ha) & $\%$ change & Area (ha) & $\%$ change & Area (ha) & $\%$ change \\
\hline Tree/shrub & 36.6 & 36.6 & 0.0 & 36.5 & -0.3 & 36.5 & -0.3 & 26.0 & -29.0 \\
\hline Casuarina equisetifolia & 65.4 & 65.3 & -0.2 & 65.3 & -0.2 & 65.0 & -0.6 & 40.9 & -37.5 \\
\hline Tournefortia argentea & 4.4 & 4.4 & 0.0 & 4.4 & 0.0 & 4.4 & 0.0 & 4.4 & 0.0 \\
\hline Mixed shrub & 16.6 & 16.6 & 0.0 & 16.6 & 0.0 & 16.5 & -0.6 & 11.9 & -28.3 \\
\hline Bare ground & 16.7 & 16.6 & -0.6 & 16.3 & -2.4 & 15.8 & -5.4 & 12.1 & -27.5 \\
\hline Beach & 22.3 & 18.1 & -18.8 & 15.3 & -31.4 & 11.4 & -48.9 & 6.4 & -71.3 \\
\hline Wetland (standing water) & 2.2 & 2.2 & 0.0 & 2.2 & 0.0 & 2.2 & 0.0 & 0.9 & -59.1 \\
\hline Human structures & 129.3 & 129.3 & 0.0 & 129.3 & 0.0 & 128.3 & -0.8 & 65.7 & -49.2 \\
\hline Total island size & 456.8 & 452.2 & -1.0 & 448.7 & -1.8 & 442.2 & -3.2 & 276.8 & -39.4 \\
\hline
\end{tabular}

Table 1.16. Total land area and percent change in area for each of six land cover types at Spit Island, Midway Atoll, with four sea-level rise (SLR) scenarios $(+0.5,+1.0,+1.5$, and +2.0 meters).

[Areas calculated from Digital Globe QuickBird satellite imagery (March 2009). Abbreviations: $m$, meters; ha, hectares; \%, percent]

\begin{tabular}{|c|c|c|c|c|c|c|c|c|c|}
\hline \multirow[b]{3}{*}{ Land cover } & \multirow{3}{*}{$\frac{0.0 \mathrm{~m}}{\text { Area (ha) }}$} & \multicolumn{8}{|c|}{ Change from SLR } \\
\hline & & \multicolumn{2}{|c|}{$+0.5 \mathrm{~m}$} & \multicolumn{2}{|c|}{$+1.0 \mathrm{~m}$} & \multicolumn{2}{|c|}{$+1.5 \mathrm{~m}$} & \multicolumn{2}{|c|}{$+2.0 \mathrm{~m}$} \\
\hline & & Area (ha) & $\%$ change & Area (ha) & $\%$ change & Area (ha) & $\%$ change & Area (ha) & $\%$ change \\
\hline Mixed shrub & 0.8 & 0.8 & 0.0 & 0.4 & -50.0 & 0.0 & -100 & 0.0 & -100 \\
\hline Grass/herbaceous cover & 1.1 & 1.1 & 0.0 & 0.3 & -72.7 & 0.0 & -100 & 0.0 & -100 \\
\hline Vine/ground cover & 0.2 & 0.2 & 0.0 & 0.1 & -50.0 & 0.0 & -100 & 0.0 & -100 \\
\hline Bare ground & 2.3 & 2.2 & -4.3 & 0.6 & -73.9 & 0.0 & -100 & 0.0 & -100 \\
\hline Beach & 0.8 & 0.2 & -75.0 & 0.0 & -100 & 0.0 & -100 & 0.0 & -100 \\
\hline Wetland (standing water) & 0.1 & 0.1 & 0.0 & 0.0 & -100 & 0.0 & -100 & 0.0 & -100 \\
\hline Total island size & 5.3 & 4.6 & -13.2 & 1.4 & -73.6 & 0.0 & -100 & 0.0 & -100 \\
\hline
\end{tabular}

Table 1.17. Total land area and percent change for each of five land cover types at Eastern Island, Midway Atoll with four sea-level rise $(S L R)$ scenarios $(+0.5,+1.0,+1.5$, and +2.0 meters).

[Areas calculated from Digital Globe QuickBird satellite imagery (March 2009). Abbreviations: $m$, meters; ha, hectares; \%, percent]

\begin{tabular}{|c|c|c|c|c|c|c|c|c|c|}
\hline \multirow[b]{3}{*}{ Land cover } & \multirow{3}{*}{$\begin{array}{c}0.0 \mathrm{~m} \\
\text { Area (ha) }\end{array}$} & \multicolumn{8}{|c|}{ Change from SLR } \\
\hline & & \multicolumn{2}{|c|}{$+0.5 \mathrm{~m}$} & \multicolumn{2}{|c|}{$+1.0 \mathrm{~m}$} & \multicolumn{2}{|c|}{$+1.5 \mathrm{~m}$} & \multicolumn{2}{|c|}{$+2.0 \mathrm{~m}$} \\
\hline & & Area (ha) & $\%$ change & Area (ha) & $\%$ change & Area (ha) & $\%$ change & Area (ha) & $\%$ change \\
\hline Tree/shrub & 16.1 & 16.1 & 0.0 & 16.1 & 0.0 & 15.7 & -2.5 & 11.1 & -31.1 \\
\hline Grass/herbaceous cover & 74.4 & 74.3 & -0.1 & 74.2 & -0.3 & 71.2 & -4.3 & 46.1 & -38.0 \\
\hline Partially vegetated former runway & 31.6 & 31.6 & 0.0 & 31.5 & -0.3 & 31.5 & -0.2 & 23 & -27.2 \\
\hline Bare ground & 1.3 & 1.2 & -7.7 & 1.1 & -15.4 & 0.8 & -38.5 & 0.3 & -76.9 \\
\hline Beach & 10.2 & 8.2 & -19.9 & 5.6 & -45.1 & 2.8 & -72.4 & 0.7 & -93.1 \\
\hline Total island size & 133.6 & 131.4 & -1.7 & 128.5 & -3.8 & 122.1 & -8.6 & 81.2 & -39.2 \\
\hline
\end{tabular}




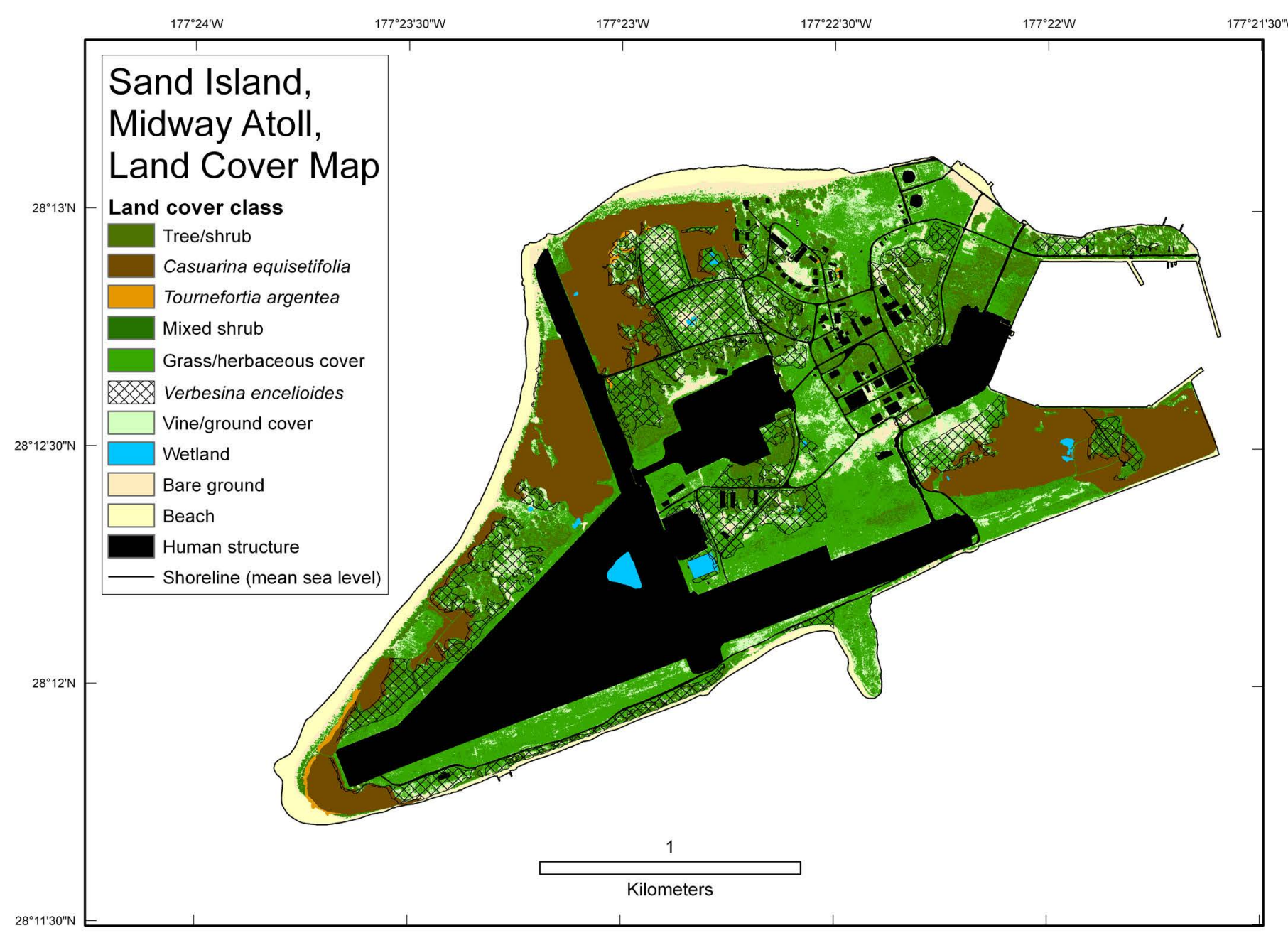

Figure 1.9. Sand Island, Midway Atoll, land cover classification map developed using primarily unsupervised classification methods from Digital Globe QuickBird satellite imagery (March 2009). Casuarina equisetifolia, Tournefortia argentea, Verbesina encelioides distribution mapped from historical field data (Laniawe, 2004). Additional details on land cover and species composition are given in table 1.15. Active management to remove C. equisetifolia, T. argentea, and V. encelioides has substantially altered the vegetation distribution. Land cover classification derived from 2011 imagery will be available in Storlazzi and others (2012, in prep., U.S. Geological Survey Open-File Report). 


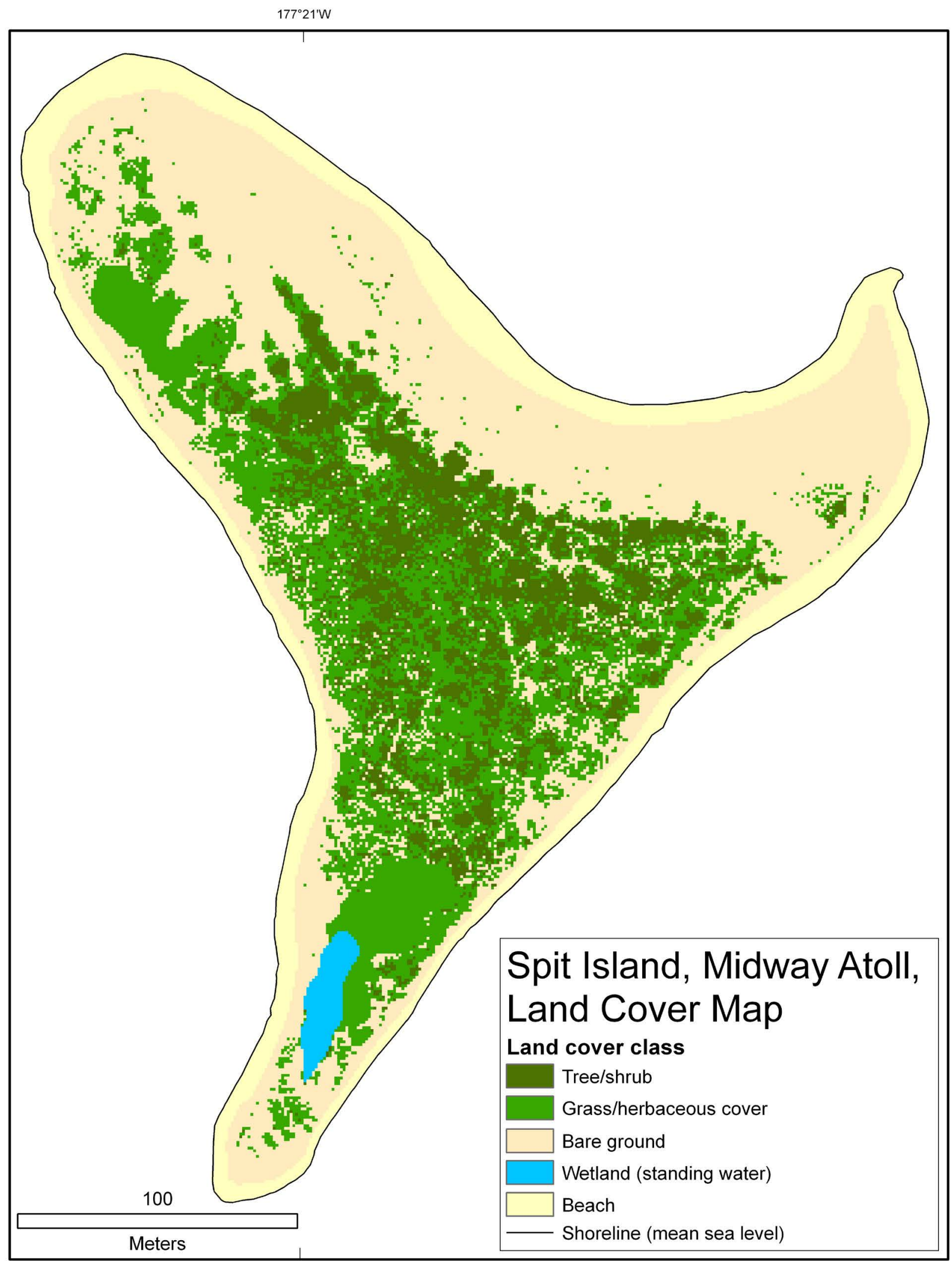

Figure 1.10. Spit Island, Midway Atoll, land cover classification map developed using primarily unsupervised classification methods from Digital Globe QuickBird satellite imagery (March 2009). Additional details on land cover are given in table 1.16. 


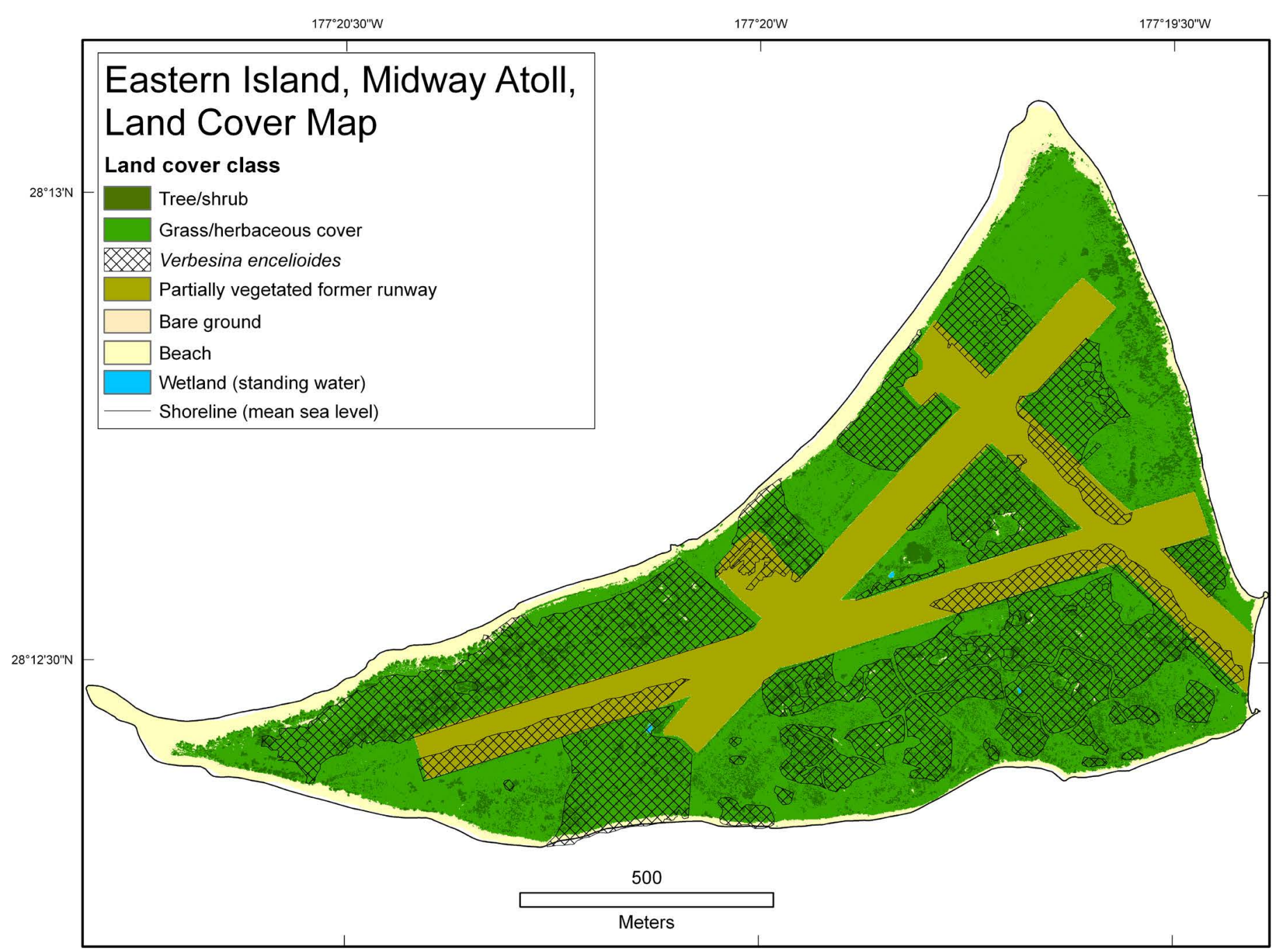

Figure 1.11. Eastern Island, Midway Atoll, land cover classification map developed using primarily unsupervised classification methods from Digital Globe QuickBird satellite imagery (March 2009). Verbesina encelioides distribution mapped from historical field data (Laniawe, 2004). Additional details on land cover and species composition are given in table 1.17 . 


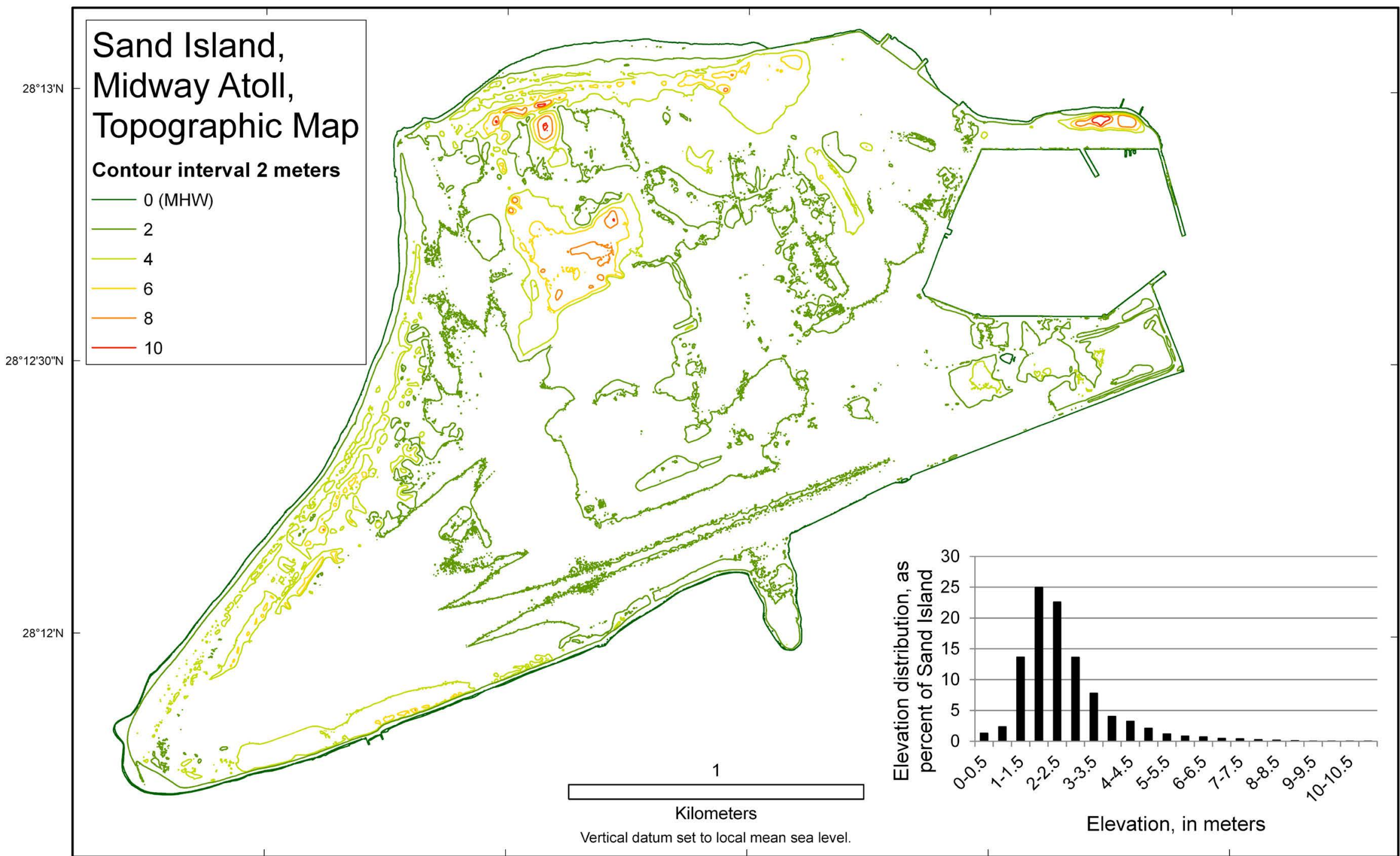

Figure 1.12. Topographic map of Sand Island, Midway Atoll, showing mean high water (MHW) and 2-meter $(\mathrm{m})$ contour lines with a graph of the percentage of land in 0.5-m elevation bins. Elevations were determined from U.S. Geological Survey lidar data (2010) with a vertical root-meansquared error of $0.05 \mathrm{~m}$. 

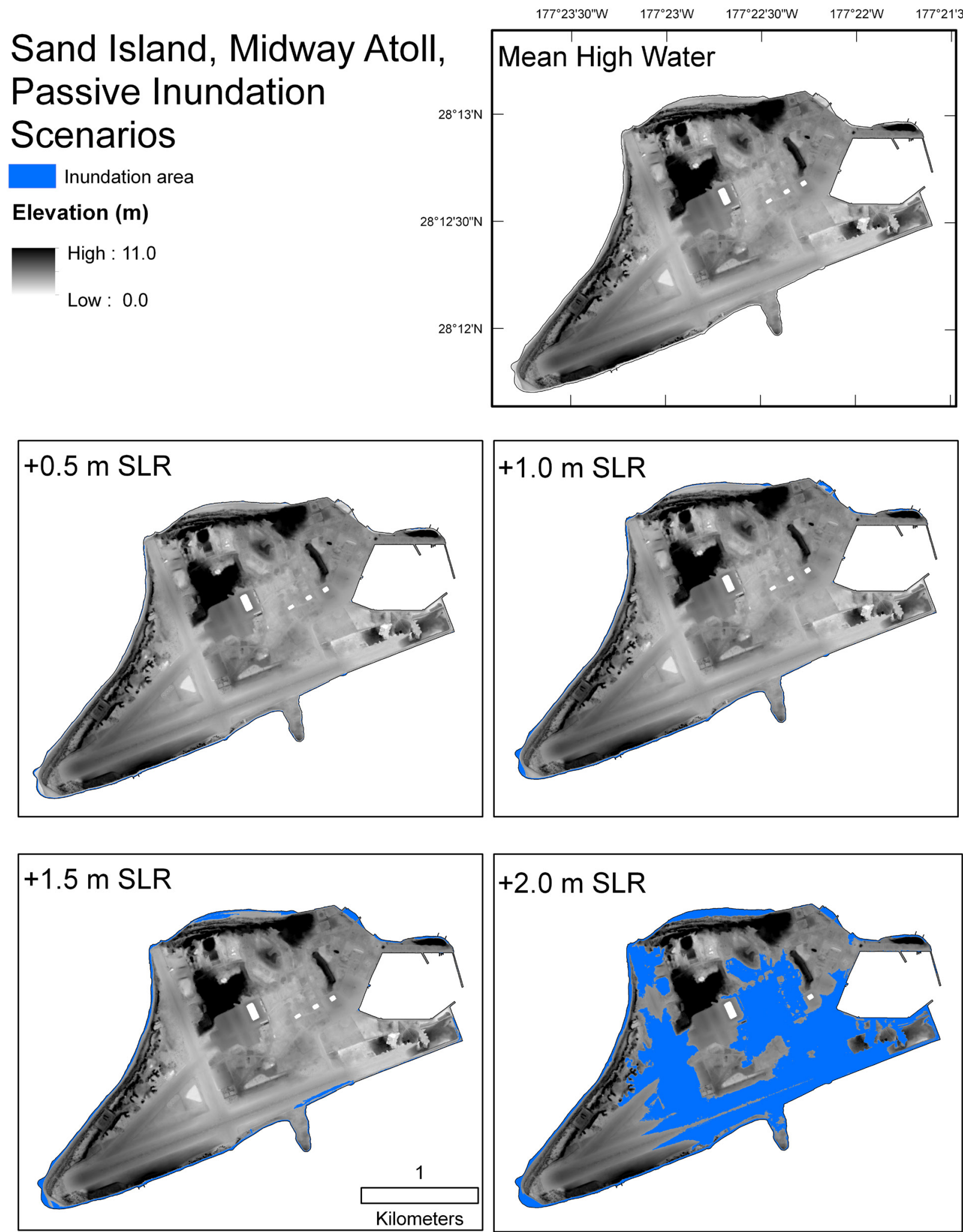

Figure 1.13. Sand Island, Midway Atoll, passive inundation scenario maps for five sea levels: mean high water through $+2.0 \mathrm{~m}$ (meters) sea-level rise (SLR) at 0.5-m increments. Inundation scenarios are based on U.S. Geological Survey lidar-derived elevations with a root-mean-squared error of $0.05 \mathrm{~m}$. 
Table 1.18. Total potential nesting habitat area and percent change in nesting habitat area for breeding avifauna at Sand Island, Midway Atoll, with four sea-level rise (SLR) scenarios $(+0.5$, $+1.0,+1.5$, and +2.0 meters).

[Areas calculated from land cover classes with Digital Globe QuickBird satellite imagery (March 2009). We assumed percent change based on a uniform density across nesting habitat for each species. Abbreviations: $\mathrm{m}$, meters; ha, hectares]

\begin{tabular}{|c|c|c|c|c|c|}
\hline \multirow[b]{2}{*}{ Species } & \multirow{2}{*}{$\begin{array}{c}\text { Area (ha) } \\
0.0 \mathrm{~m}\end{array}$} & \multicolumn{4}{|c|}{ Percent Change from SLR } \\
\hline & & $+0.5 \mathrm{~m}$ & $+1.0 \mathrm{~m}$ & $+1.5 \mathrm{~m}$ & $+2.0 \mathrm{~m}$ \\
\hline Black-footed Albatross & 262.0 & -0.2 & -0.4 & -1.0 & -33.8 \\
\hline Laysan Albatross & 262.0 & -0.2 & -0.4 & -1.0 & -33.8 \\
\hline Bonin Petrel & 286.3 & -0.1 & -0.2 & -0.6 & -33.0 \\
\hline Wedge-tailed Shearwater & 303.0 & -0.1 & -0.4 & -0.9 & -32.7 \\
\hline Red-tailed Tropicbird & 256.8 & -0.1 & -0.2 & -0.5 & -32.7 \\
\hline White-tailed Tropicbird & 65.4 & -0.2 & -0.2 & -0.6 & -37.5 \\
\hline Little Tern & 180.0 & -0.2 & -0.5 & -1.2 & -33.0 \\
\hline Brown Noddy & 303.0 & -0.1 & -0.4 & -0.9 & -32.7 \\
\hline Black Noddy & 123.0 & -0.1 & -0.2 & -0.5 & -32.4 \\
\hline White Tern & 129.0 & -0.1 & -0.2 & -0.5 & -33.0 \\
\hline Laysan Teal & 286.3 & -0.1 & -0.2 & -0.6 & -33.0 \\
\hline
\end{tabular}

Table 1.19. Total potential nesting habitat area and percent change in nesting habitat area for breeding avifauna at Spit Island, Midway Atoll, with four sea-level rise (SLR) scenarios (+0.5, $+1.0,+1.5$, and +2.0 meters).

[Areas calculated from land cover classes with Digital Globe QuickBird satellite imagery (March 2009). We assumed percent change based on uniform density across nesting habitat for each species. Abbreviations: $\mathrm{m}$, meters; ha, hectares]

\begin{tabular}{|c|c|c|c|c|c|}
\hline \multirow[b]{2}{*}{ Species } & \multirow{2}{*}{$\begin{array}{c}\text { Area (ha) } \\
0.0 \mathrm{~m}\end{array}$} & \multicolumn{4}{|c|}{ Percent Change from SLR } \\
\hline & & $+0.5 \mathrm{~m}$ & $+1.0 \mathrm{~m}$ & $+1.5 \mathrm{~m}$ & $+2.0 \mathrm{~m}$ \\
\hline Black-footed Albatross & 4.4 & -2.3 & -68.2 & -100 & -100 \\
\hline Laysan Albatross & 4.4 & -2.3 & -68.2 & -100 & -100 \\
\hline Bonin Petrel & 2.1 & 0.0 & -61.9 & -100 & -100 \\
\hline Christmas Shearwater & 2.1 & 0.0 & -61.9 & -100 & -100 \\
\hline Red-tailed Tropicbird & 1.9 & 0.0 & -63.2 & -100 & -100 \\
\hline Masked Booby & 3.6 & -2.8 & -72.2 & -100 & -100 \\
\hline Brown Booby & 3.6 & -2.8 & -72.2 & -100 & -100 \\
\hline Red-footed Booby & 0.8 & 0.0 & -50.0 & -100 & -100 \\
\hline Great Frigatebird & 0.8 & 0.0 & -50.0 & -100 & -100 \\
\hline Gray-backed Tern & 2.5 & -4.0 & -72.0 & -100 & -100 \\
\hline Brown Noddy & 4.4 & -2.3 & -68.2 & -100 & -100 \\
\hline
\end{tabular}




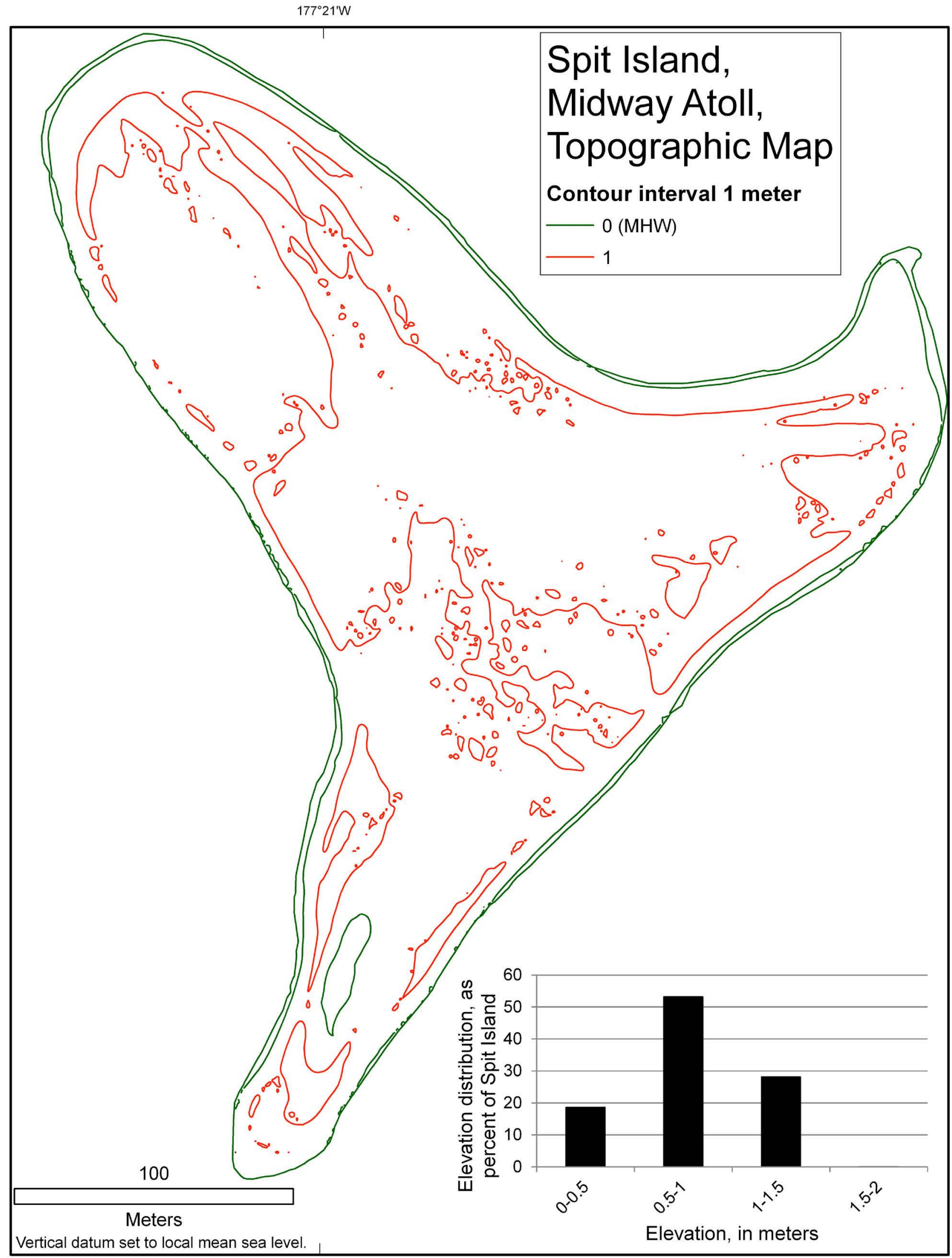

Figure 1.14. Topographic map of Spit Island, Midway Atoll, showing mean high water (MHW) and 1-meter (m) contour lines with a graph of the percentage of land in $0.5-\mathrm{m}$ elevation bins. Elevations were determined from U.S. Geological Survey lidar data (2010) with a vertical root-mean-squared error of $0.05 \mathrm{~m}$. 
Spit Island, Midway Atoll, Passive Inundation Scenarios

Inundation area

\section{Elevation (m)}

High : 1.8

Low : 0.0
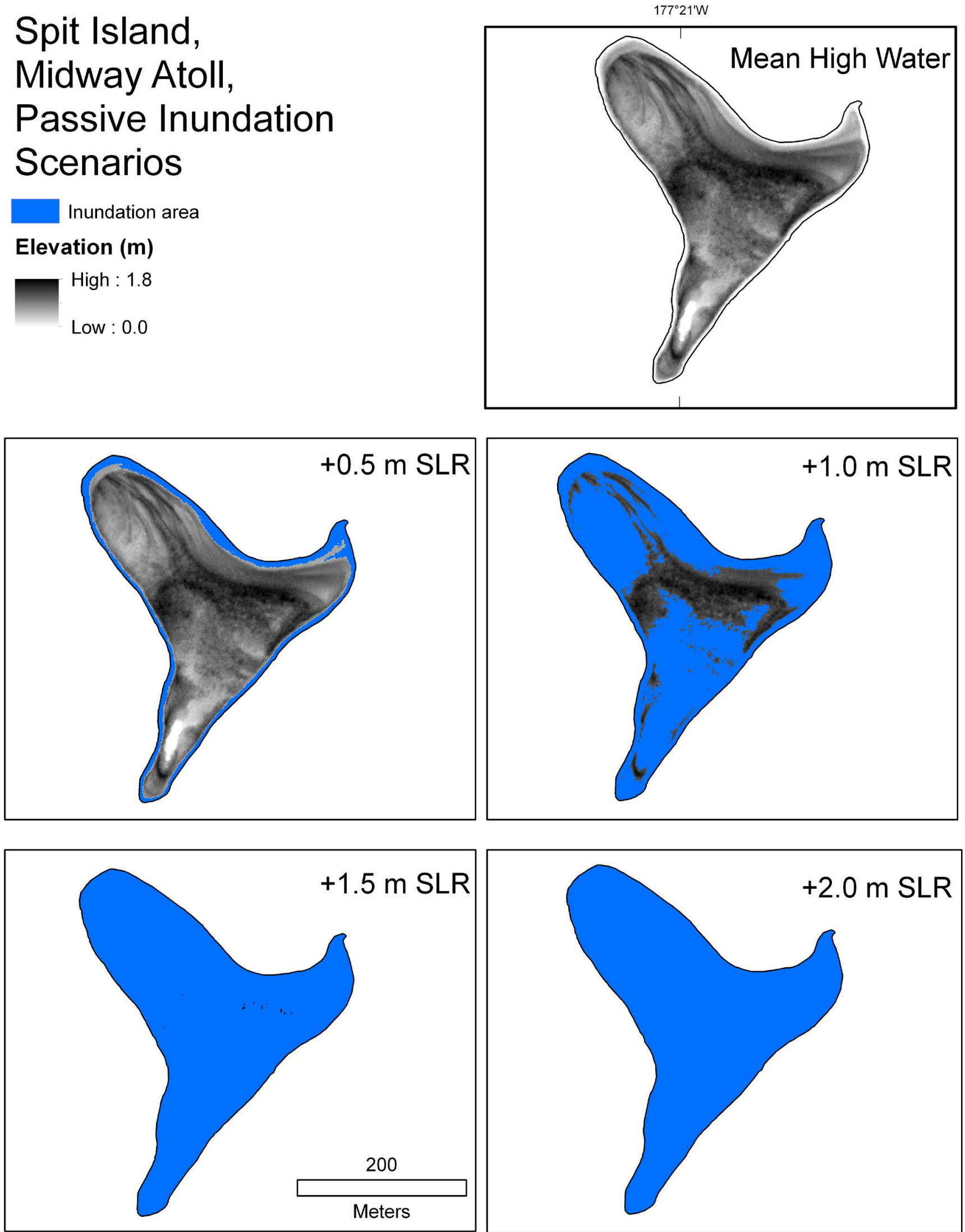

Figure 1.15. Spit Island, Midway Atoll, passive inundation scenario maps for five sea levels: mean high water through $+2.0 \mathrm{~m}$ (meters) sea-level rise (SLR) at 0.5-m increments. Inundation scenarios are based on U.S. Geological Survey lidar-derived elevations with a root-mean-squared error of $0.05 \mathrm{~m}$. 
Table 1.20. Total potential nesting habitat area and percent change for breeding avifauna at Eastern Island, Midway Atoll, with four sea-level rise (SLR) scenarios $(+0.5,+1.0,+1.5$, and +2.0 meters).

[Areas calculated from land cover classes with Digital Globe QuickBird satellite imagery (March 2009). We assumed percent change from a uniform density across nesting habitat for each species. Abbreviations: $\mathrm{m}$, meters; ha, hectares]

\begin{tabular}{lcccccc}
\hline & Area (ha) & & \multicolumn{3}{c}{ Percent Change from SLR } \\
\cline { 2 - 3 } \cline { 5 - 6 } Species & $\mathbf{0 . 0} \mathbf{~}$ & & $\mathbf{+ 0 . 5} \mathbf{~}$ & $\mathbf{+ 1 . 0} \mathbf{~ m}$ & $\mathbf{+ 1 . 5} \mathbf{~}$ & $\mathbf{+ 2 . 0} \mathbf{~ m}$ \\
\hline Black-footed Albatross & 107.3 & & -0.2 & -0.5 & -3.5 & -35.3 \\
Laysan Albatross & 107.3 & & -0.2 & -0.5 & -3.5 & -35.3 \\
Short-tailed Albatross & 107.3 & & -0.2 & -0.5 & -3.5 & -35.3 \\
Bonin Petrel & 90.5 & & -0.1 & -0.2 & -4.0 & -36.8 \\
Wedge-tailed Shearwater & 91.8 & & -0.2 & -0.4 & -4.5 & -37.4 \\
Christmas Shearwater & 122.1 & & -0.1 & -0.2 & -3.0 & -34.3 \\
Red-tailed Tropicbird & 122.1 & & -0.1 & -0.2 & -3.0 & -34.3 \\
Masked Booby & 107.3 & & -0.2 & -0.5 & -3.5 & -35.3 \\
Brown Booby & 107.3 & & -0.2 & -0.5 & -3.5 & -35.3 \\
Red-footed Booby & 16.1 & & 0.0 & 0.0 & -2.5 & -31.1 \\
Great Frigatebird & 16.1 & & 0.0 & 0.0 & -2.5 & -31.1 \\
Gray-backed Tern & 32.9 & -0.3 & -0.9 & -1.8 & -29.2 \\
Sooty Tern & 107.3 & -0.2 & -0.5 & -3.5 & -35.3 \\
Brown Noddy & 123.4 & -0.2 & -0.4 & -3.4 & -34.8 \\
Black Noddy & 16.1 & 0.0 & 0.0 & -2.5 & -31.1 \\
White Tern & 16.1 & 0.0 & 0.0 & -2.5 & -31.1 \\
Laysan Teal & 90.5 & -0.1 & -0.2 & -4.0 & -36.8 \\
\hline
\end{tabular}




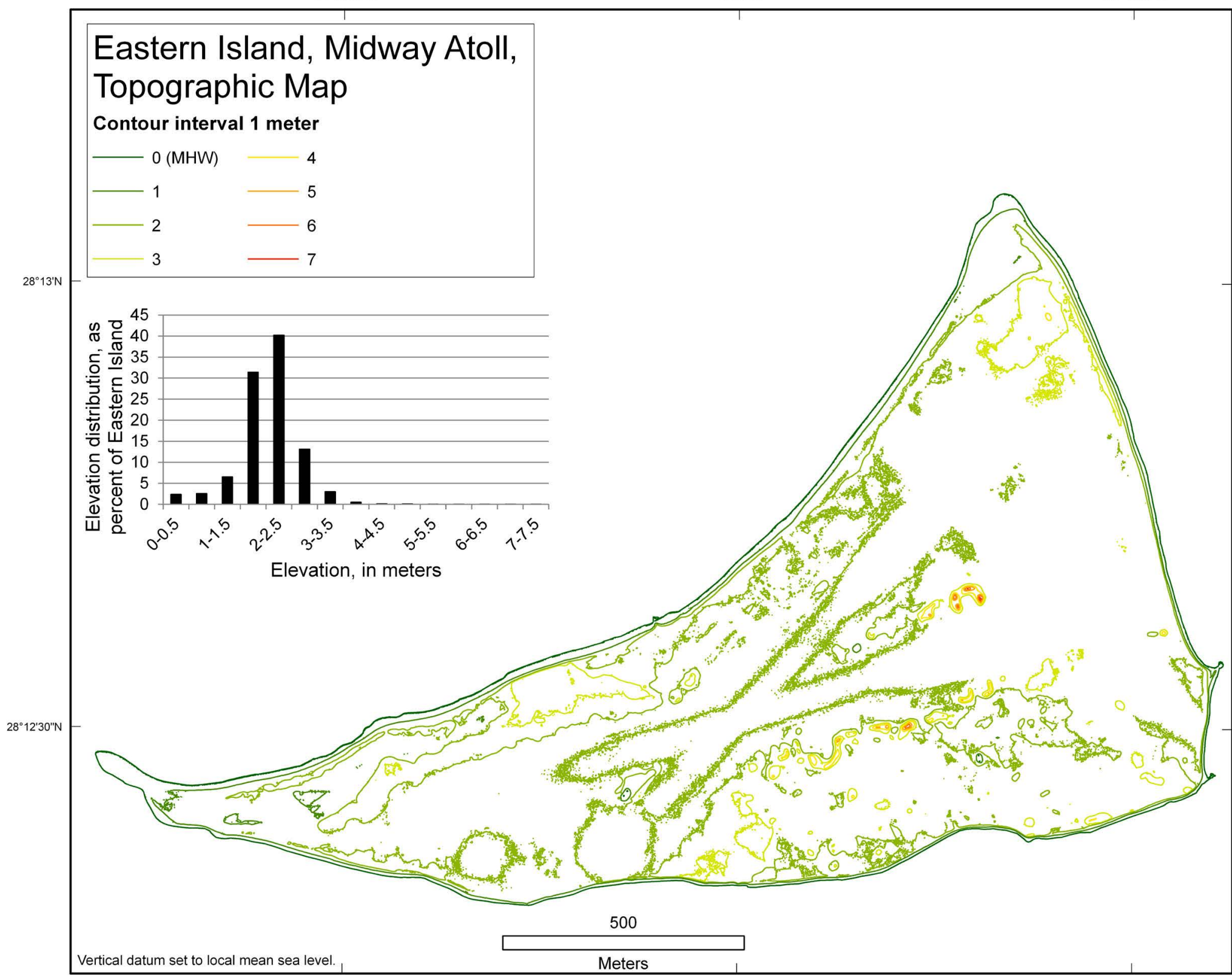

Figure 1.16. Topographic map of Eastern Island, Midway Atoll, showing mean high water (MHW) and 1-meter $(\mathrm{m})$ contour lines with a graph of the percentage of land in 0.5-m elevation bins. Elevations were determined from U.S. Geological Survey lidar data (2010) with a vertical root-mean-squared error of $0.05 \mathrm{~m}$. 
Eastern Island, Midway Atoll, Passive Inundation Scenarios

Inundation area

\section{Elevation (m)}

High : 7.5

Low : 0.0
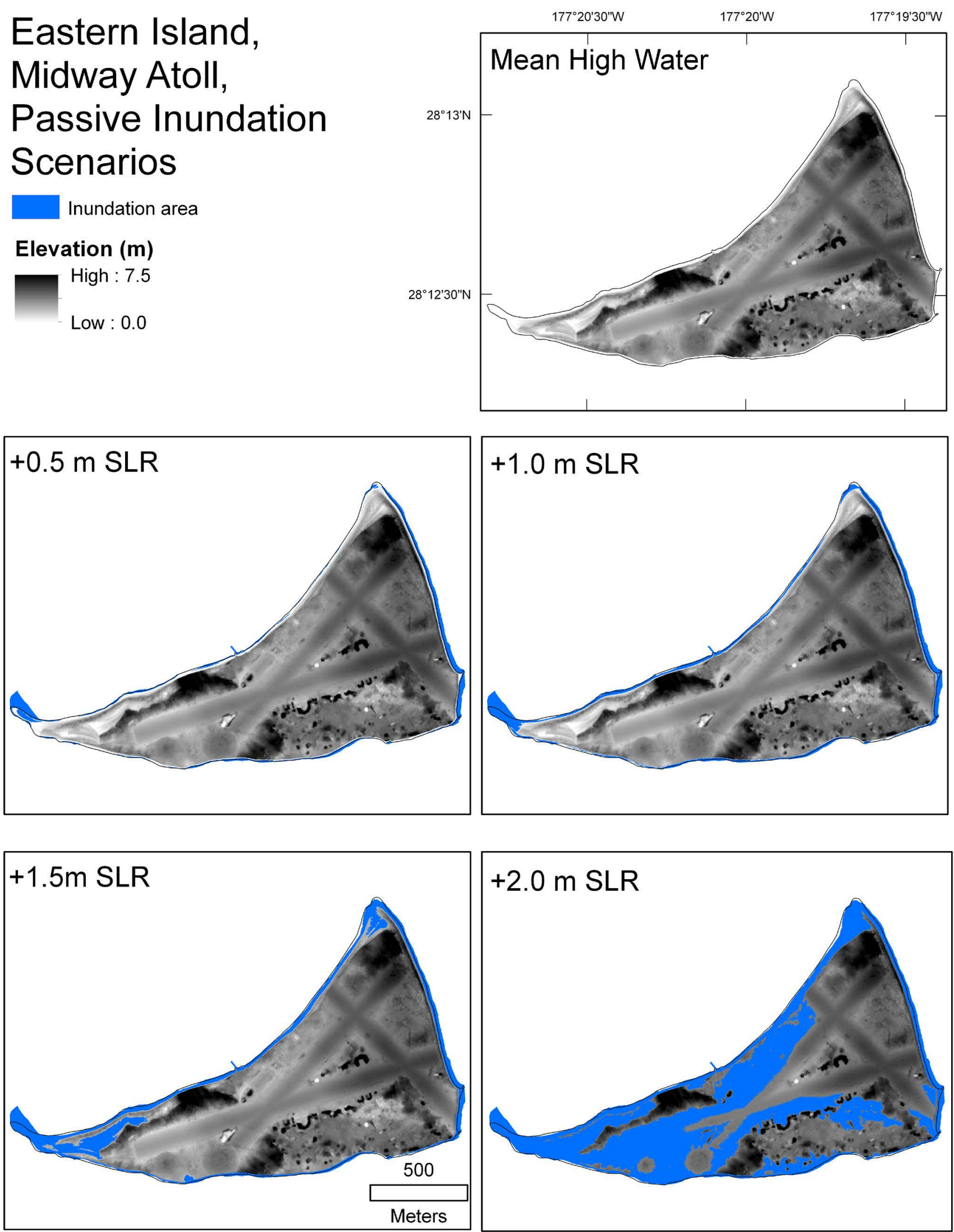

\section{$+2.0 \mathrm{~m} \mathrm{SLR}$}

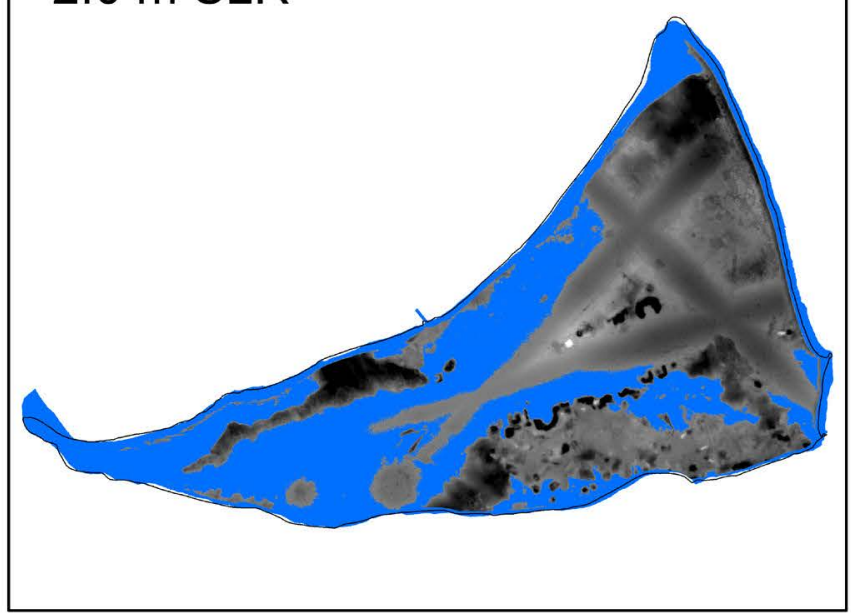

Figure 1.17. Eastern Island, Midway Atoll, passive inundation scenario maps for five sea levels: mean high water through +2.0 meters $(\mathrm{m})$ sea-level rise (SLR) at 0.5-m increments. Inundation scenarios are based on U.S. Geological Survey lidar-derived elevations with a root-mean-squared error of $0.05 \mathrm{~m}$. 


\section{Pearl and Hermes Atoll}

\section{Island Information}

North, Little North, Southeast, Grass, and Seal-Kittery Islands and several small sand spits constitute Pearl and Hermes Atoll (fig. 1.18). Southeast Island was the largest island of the atoll (18.4 ha), followed by Seal-Kittery Island (13.7 ha), North Island (8.4 ha), Little North Island (3.2 ha), and Grass Island (3.2 ha; table 1.9). Vegetation was only found on the three largest islands: North, Southeast, and
Seal-Kittery. Land cover consisted of six classes: grass/ herbaceous cover including Cenchrus echinatus, Cynodon dactylon, Eragrostis paupera, Eragrostis variabilis, Lepturus repens, Setaria verticillata, Solanum americanum, Solanum nelsonii, Sonchus oleraceus, Verbesina encelioides; vine/ground cover including Boerhavia repens, Coronopus didymus, Ipomoea pes-caprae, Portulaca lutea, Portulaca oleracea, Sicyos maximowiczii, and Tribulus cistoides; wetland vegetation including Sesuvium portulacastrum; bare ground; beach; and wetland (standing water; tables 1.21-25, figs. 1.19-21). The dominant land cover of the atoll was beach totaling 23.9 ha across the islands (tables 1.21-25).

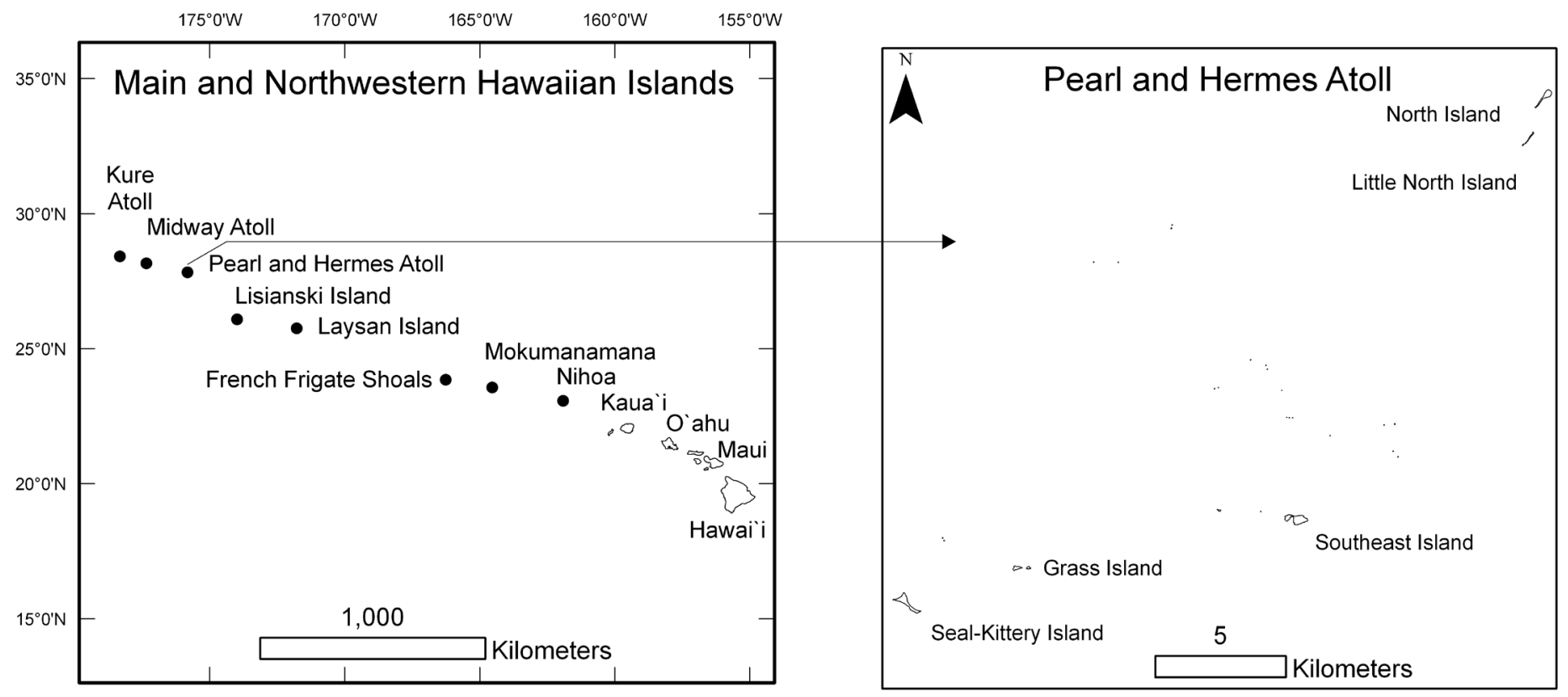

Figure 1.18. Maps showing the location of Pearl and Hermes Atoll, a coral atoll located between Midway Atoll to the northwest and Lisianski Island to the southeast. Pearl and Hermes Atoll comprises multiple sand spits and five islands: North, Little North, Southeast, Grass, and Seal-Kittery. 


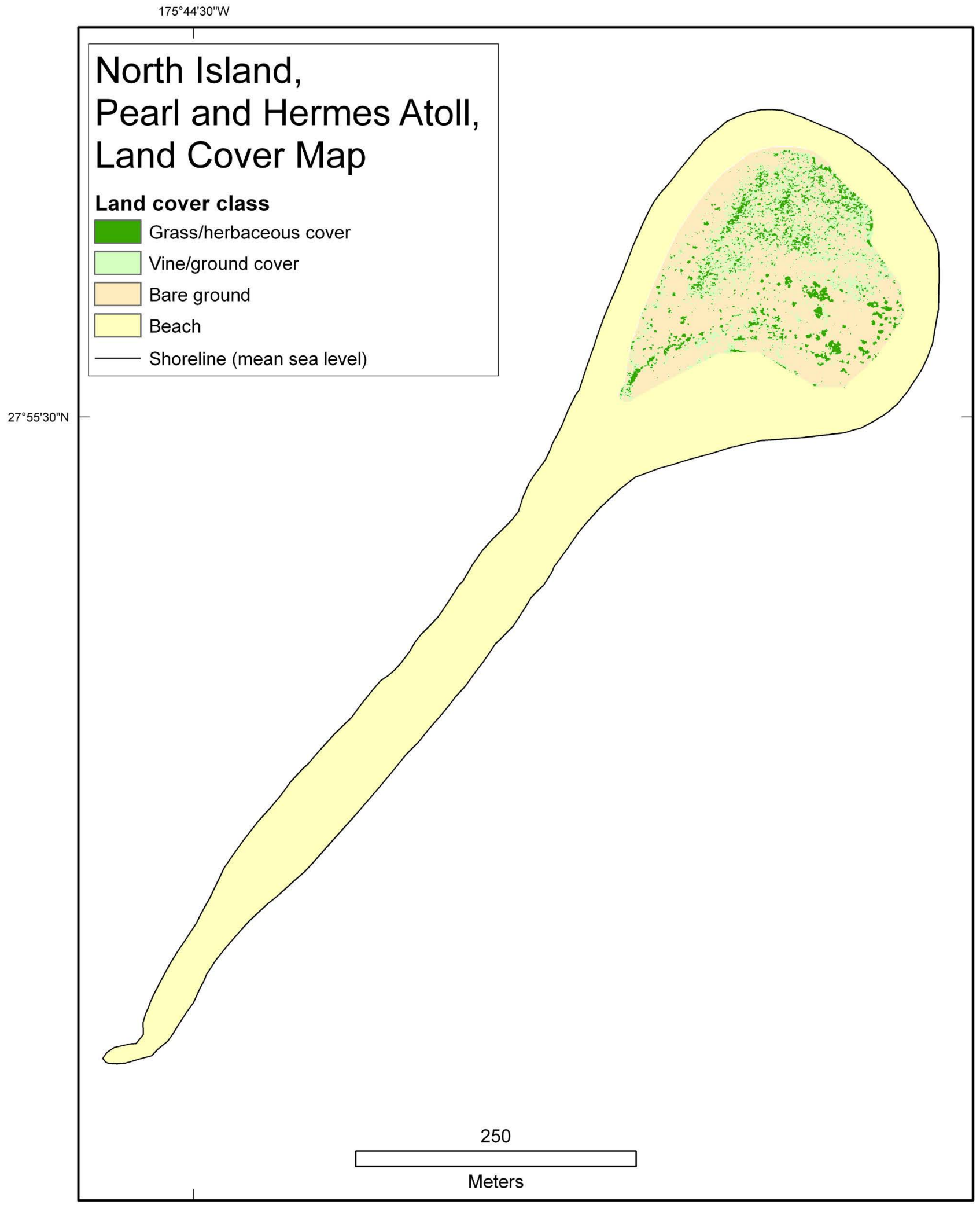

Figure 1.19. North Island, Pearl and Hermes Atoll, land cover classification map developed using primarily unsupervised classification methods from Digital Globe QuickBird satellite imagery (October 2007). Additional details on land cover are given in table 1.21. 
$175^{\circ} 49^{\prime} \mathrm{W}$

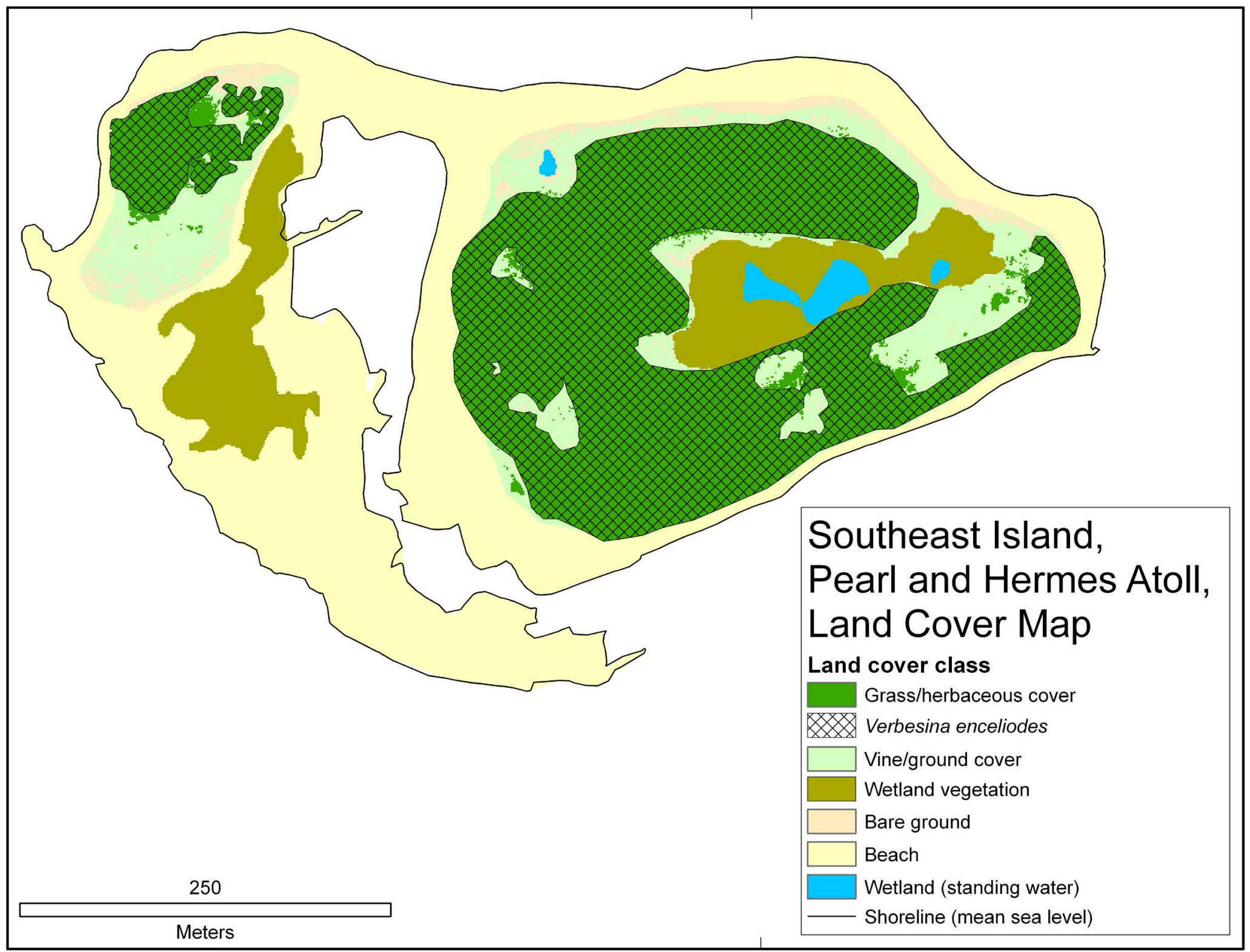

Figure 1.20. Southeast Island, Pearl and Hermes Atoll, land cover classification map developed using primarily unsupervised classification methods from Digital Globe QuickBird satellite imagery (October 2007). Verbesina encelioides distribution was mapped based on summer 2003 field data (Sprague 2003). Additional details on land cover and species composition are given in table 1.23. 


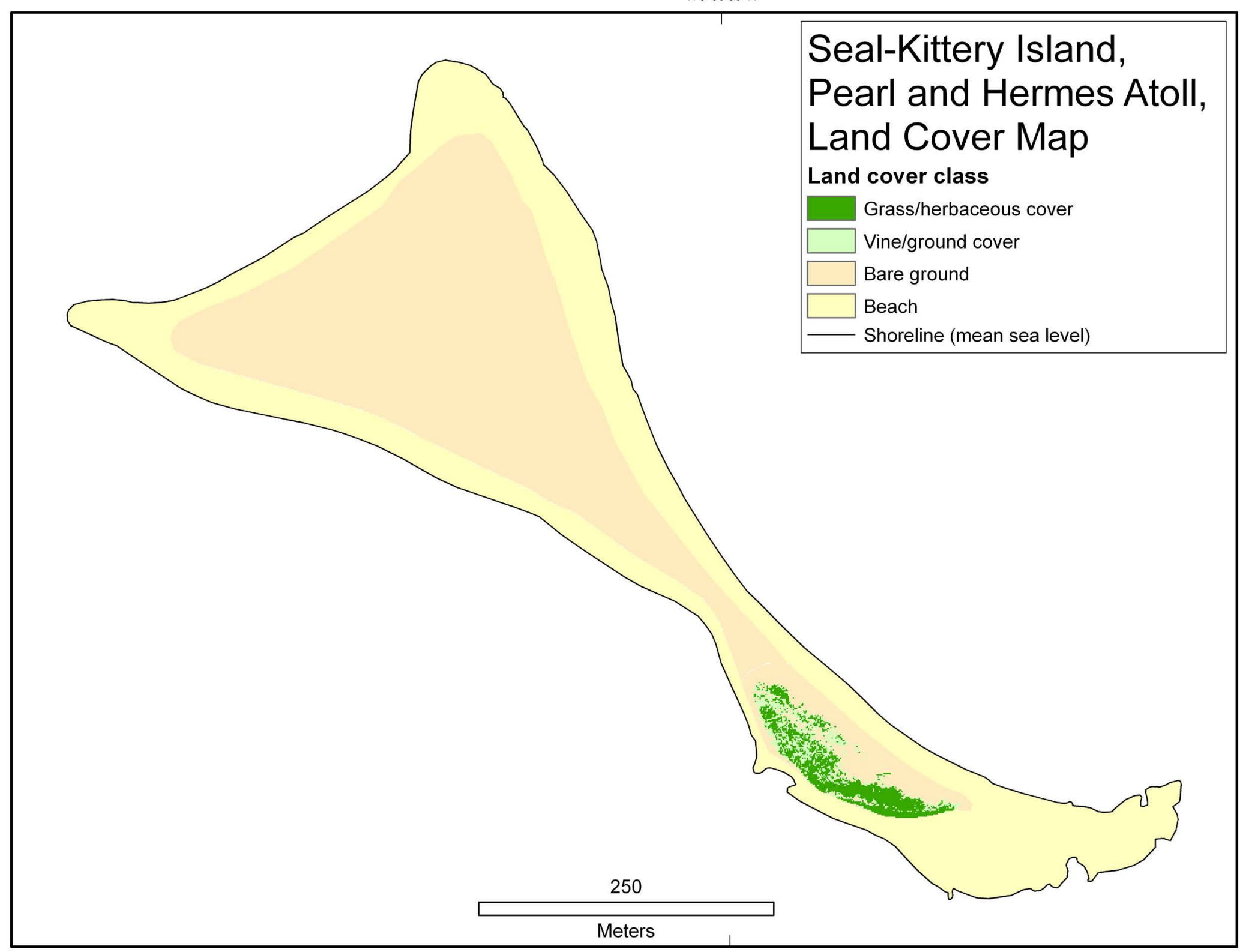

Figure 1.21. Seal-Kittery Island, Pearl and Hermes Atoll, land cover classification map developed using primarily unsupervised classification methods from Digital Globe QuickBird satellite imagery (October 2007). Additional details on land cover are given in table 1.25. 
Table 1.21. Total land area and percent change for each of four land cover types at North Island, Pearl and Hermes Atoll, with four sea-level rise (SLR) scenarios $(+0.5,+1.0,+1.5$, and +2.0 meters).

[Areas calculated from Digital Globe QuickBird satellite imagery (October 2007). Abbreviations: m, meters; ha, hectares; \%, percent]

\begin{tabular}{|c|c|c|c|c|c|c|c|c|c|}
\hline \multirow[b]{3}{*}{ Land cover } & \multirow{3}{*}{$\begin{array}{c}0.0 \mathrm{~m} \\
\text { Area (ha) }\end{array}$} & \multicolumn{8}{|c|}{ Change from SLR } \\
\hline & & \multicolumn{2}{|c|}{$+0.5 \mathrm{~m}$} & \multicolumn{2}{|c|}{$+1.0 \mathrm{~m}$} & \multicolumn{2}{|c|}{$+1.5 \mathrm{~m}$} & \multicolumn{2}{|c|}{$+2.0 \mathrm{~m}$} \\
\hline & & Area (ha) & $\%$ change & Area (ha) & $\%$ change & Area (ha) & $\%$ change & Area (ha) & $\%$ change \\
\hline Grass/herbaceous cover & 0.2 & 0.2 & 0.0 & 0.2 & 0.0 & 0.1 & -50.0 & 0.0 & -100 \\
\hline Bare ground & 1.9 & 1.9 & 0.0 & 1.9 & 0.0 & 1.5 & -21.1 & 0.3 & -84.2 \\
\hline Beach & 5.7 & 4.8 & -15.8 & 3.7 & -35.1 & 2.3 & -59.6 & 0.5 & -91.2 \\
\hline
\end{tabular}

Table 1.22. Total land area and percent change for each of two land cover types at Little North Island, Pearl and Hermes Atoll, with four sea-level rise (SLR) scenarios $(+0.5,+1.0,+1.5$, and +2.0 meters).

[Areas calculated from Digital Globe QuickBird satellite imagery (October 2007). Abbreviations: m, meters; ha, hectares; \%, percent]

\begin{tabular}{|c|c|c|c|c|c|c|c|c|c|}
\hline \multirow[b]{3}{*}{ Land cover } & \multirow{3}{*}{$\frac{0.0 \mathrm{~m}}{\text { Area (ha) }}$} & \multicolumn{8}{|c|}{ Change from SLR } \\
\hline & & \multicolumn{2}{|c|}{$+0.5 \mathrm{~m}$} & \multicolumn{2}{|c|}{$+1.0 \mathrm{~m}$} & \multicolumn{2}{|c|}{$+1.5 \mathrm{~m}$} & \multicolumn{2}{|c|}{$+2.0 \mathrm{~m}$} \\
\hline & & Area (ha) & $\%$ change & Area (ha) & $\%$ change & Area (ha) & $\%$ change & Area (ha) & $\%$ change \\
\hline Bare ground & 0.3 & 0.3 & 0.0 & 0.3 & 0.0 & 0.3 & 0.0 & 0.0 & -100 \\
\hline Beach & 2.9 & 2.0 & -31.0 & 0.8 & -72.4 & 0.0 & -100 & 0.0 & -100 \\
\hline Total island size & 3.2 & 2.3 & -28.1 & 1.1 & -65.6 & 0.3 & -90.6 & 0.0 & -100 \\
\hline
\end{tabular}

Table 1.23. Total land area and percent change for each of six land cover types at Southeast Island, Pearl and Hermes Atoll, with four sea-level rise (SLR) scenarios $(+0.5,+1.0,+1.5$, and +2.0 meters).

[Areas calculated from Digital Globe QuickBird satellite imagery (October 2007). Abbreviations: m, meters; ha, hectares; \%, percent]

\begin{tabular}{|c|c|c|c|c|c|c|c|c|c|}
\hline \multirow[b]{3}{*}{ Land cover } & \multirow{3}{*}{$\begin{array}{c}0.0 \mathrm{~m} \\
\text { Area (ha) }\end{array}$} & \multicolumn{8}{|c|}{ Change from SLR } \\
\hline & & \multicolumn{2}{|c|}{$+0.5 \mathrm{~m}$} & \multicolumn{2}{|c|}{$+1.0 \mathrm{~m}$} & \multicolumn{2}{|c|}{$+1.5 \mathrm{~m}$} & \multicolumn{2}{|c|}{$+2.0 \mathrm{~m}$} \\
\hline & & Area (ha) & $\%$ change & Area (ha) & $\%$ change & Area (ha) & $\%$ change & Area (ha) & $\%$ change \\
\hline Grass/herbaceous cover/Verbesina encelioides & 6.6 & 6.6 & 0.0 & 5.1 & -22.7 & 2.3 & -65.2 & 0.4 & -93.9 \\
\hline Vine/ground cover & 2.0 & 2.0 & 0.0 & 1.5 & -25.0 & 0.7 & -65.0 & 0.1 & -95.0 \\
\hline Wetland vegetation & 1.9 & 1.4 & -26.3 & 0.0 & -100 & 0.0 & -100 & 0.0 & -100 \\
\hline Bare ground & 0.5 & 0.5 & 0.0 & 0.3 & -40.0 & 0.1 & -80.0 & 0.0 & -100 \\
\hline Beach & 7.2 & 3.4 & -52.8 & 0.8 & -88.9 & 0.1 & -98.6 & 0.0 & -100 \\
\hline Wetland (standing water) & 0.2 & 0.2 & 0.0 & 0.0 & -100 & 0.0 & -100 & 0.0 & -100 \\
\hline Total island size & 18.4 & 14.1 & -23.4 & 7.7 & -58.2 & 3.2 & -82.6 & 0.5 & -97.3 \\
\hline
\end{tabular}


Table 1.24. Total land area and percent change for each of three land cover types at Grass Island, Pearl and Hermes Atoll, with four sea-level rise (SLR) scenarios $(+0.5,+1.0,+1.5$, and +2.0 meters).

[Areas calculated from Digital Globe QuickBird satellite imagery (October 2007). Abbreviations: m, meters; ha, hectares; \%, percent]

\begin{tabular}{|c|c|c|c|c|c|c|c|c|c|}
\hline \multirow[b]{3}{*}{ Land cover } & \multirow{3}{*}{$\frac{0.0 \mathrm{~m}}{\text { Area (ha) }}$} & \multicolumn{8}{|c|}{ Change from SLR } \\
\hline & & \multicolumn{2}{|c|}{$+0.5 \mathrm{~m}$} & \multicolumn{2}{|c|}{$+1.0 \mathrm{~m}$} & \multicolumn{2}{|c|}{$+1.5 \mathrm{~m}$} & \multicolumn{2}{|c|}{$+2.0 \mathrm{~m}$} \\
\hline & & Area (ha) & $\%$ change & Area (ha) & $\%$ change & Area (ha) & $\%$ change & Area (ha) & $\%$ change \\
\hline Grass/herbaceous cover & 0.5 & 0.5 & 0.0 & 0.5 & 0.0 & 0.5 & 0.0 & 0.2 & -60.0 \\
\hline Beach & 2.0 & 1.6 & -19.6 & 1.0 & -50.0 & 0.1 & -95.0 & 0.0 & -100 \\
\hline Total island size & 3.2 & 2.8 & -13.3 & 2.2 & -31.3 & 1.2 & -62.8 & 0.3 & -90.6 \\
\hline
\end{tabular}

Table 1.25. Total land area and percent change for each of four land cover types at Seal-Kittery Island, Pearl and Hermes Atoll, with four sea-level rise (SLR) scenarios $(+0.5,+1.0,+1.5$, and +2.0 meters).

[Areas calculated from Digital Globe QuickBird satellite imagery (October 2007). Abbreviations: m, meters; ha, hectares; \%, percent]

\begin{tabular}{|c|c|c|c|c|c|c|c|c|c|}
\hline \multirow[b]{3}{*}{ Land cover } & \multirow{3}{*}{$\begin{array}{c}0.0 \mathrm{~m} \\
\text { Area (ha) }\end{array}$} & \multicolumn{8}{|c|}{ Change from SLR } \\
\hline & & \multicolumn{2}{|c|}{$+0.5 \mathrm{~m}$} & \multicolumn{2}{|c|}{$+1.0 \mathrm{~m}$} & \multicolumn{2}{|c|}{$+1.5 \mathrm{~m}$} & \multicolumn{2}{|c|}{$+2.0 \mathrm{~m}$} \\
\hline & & Area (ha) & $\%$ change & Area (ha) & $\%$ change & Area (ha) & $\%$ change & Area (ha) & $\%$ change \\
\hline Grass/herbaceous cover & 0.3 & 0.3 & 0.0 & 0.3 & 0.0 & 0.2 & -33.3 & 0.1 & -67.7 \\
\hline Vine/ground cover & 0.2 & 0.2 & 0.0 & 0.2 & 0.0 & 0.2 & 0.0 & 0.1 & -52.4 \\
\hline Bare ground & 7.1 & 7.1 & 0.0 & 7.1 & 0.0 & 4.5 & -36.6 & 1.9 & -73.2 \\
\hline Beach & 6.1 & 3.8 & -37.7 & 1.9 & -68.9 & 0.3 & -95.1 & 0.0 & -100 \\
\hline
\end{tabular}

\section{Sea-Level Rise}

The mean elevation of the islands of Pearl and Hermes Atoll was $1.2 \mathrm{~m}$ (SD 0.2, RMSE 0.12; table 1.9). Maximum elevation of islands in the atoll ranged from $2.3 \mathrm{~m}$ at Grass Island to $2.6 \mathrm{~m}$ at Seal-Kittery Island (table 1.9). Maximum, mean, and standard deviation of elevation for all islands are presented in table 1.9. Of the total land area of Pearl and Hermes Atoll (excluding sand spits), 92 percent was below $2.0 \mathrm{~m}$ elevation. At least 85 percent of the land area of each island of the atoll was below $2.0 \mathrm{~m}$ elevation (figs. 1.22-26).

Of the low-lying atolls and islands of the NWHI, proportional land area loss was greatest at Pearl and Hermes Atoll where 43 percent of total land area was expected to be lost at $+1.0 \mathrm{~m}$ SLR and 92 percent lost at $+2.0 \mathrm{~m}$ SLR. Among the islands of Pearl and Hermes Atoll, land area losses expected from passive inundation varied (tables 1.21-25). The greatest losses in the atoll were predicted for Little North and
Southeast Islands at which 66 percent and 58 percent of total land area, respectively, was lost at $+1.0 \mathrm{~m} \mathrm{SLR}$; at $+2.0 \mathrm{~m}$ SLR more than 97 percent was lost at both islands (tables 1.22-23). Vegetated area across the atoll was expected to be reduced by 32 percent at $+1.0 \mathrm{~m}$ SLR and 92 percent at $+2.0 \mathrm{~m} \mathrm{SLR}$, while beach was expected to be reduced by 66 percent at $+1.0 \mathrm{~m} \mathrm{SLR}$ and 98 percent at $+2.0 \mathrm{~m}$ SLR (tables 1.21-25).

No seabird breeding habitat was predicted to be lost at $+0.5 \mathrm{~m}$ SLR across the atoll. For bird species at North, Little North, and Grass Islands the limited breeding habitat (2.7 ha or less; tables 1.26-30) was reduced by 60-100 percent at $+2.0 \mathrm{~m}$ SLR. At Southeast Island, breeding habitat for all species, including the endangered Laysan Finch, was reduced by 23-37 percent at $+1.0 \mathrm{~m}$ SLR and by more than 94 percent at $+2.0 \mathrm{~m}$ SLR (table 1.28). The relatively small amount of potential habitat for Bulwer's Petrel (0.5 ha) and Gray-backed Tern (2.5 ha) at Southeast Island was reduced by 40 percent and 28 percent, respectively, at $+1.0 \mathrm{~m} \mathrm{SLR}$ (table 1.28). 


\section{North Island, Pearl and Hermes Atoll, Topographic Map \\ Contour interval 1 meter

0 (MHW)
1
2
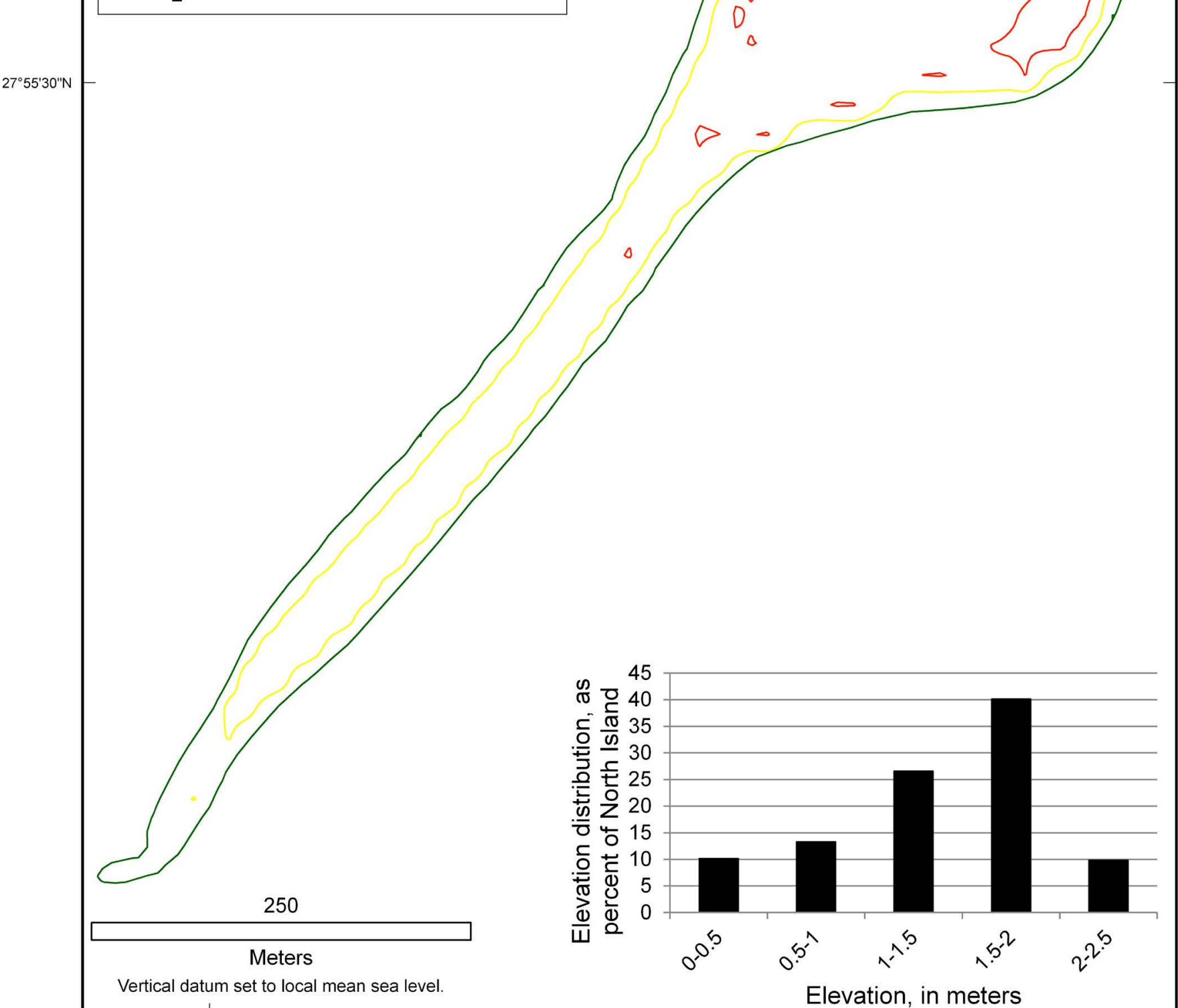

Figure 1.22. Topographic map of North Island, Pearl and Hermes Atoll, showing mean high water (MHW) and 1-meter $(\mathrm{m})$ contour lines with a graph of the percentage of land in $0.5-\mathrm{m}$ elevation bins. Elevations were determined from U.S. Geological Survey lidar data (2010) with a vertical root-mean-squared error of $0.12 \mathrm{~m}$. 


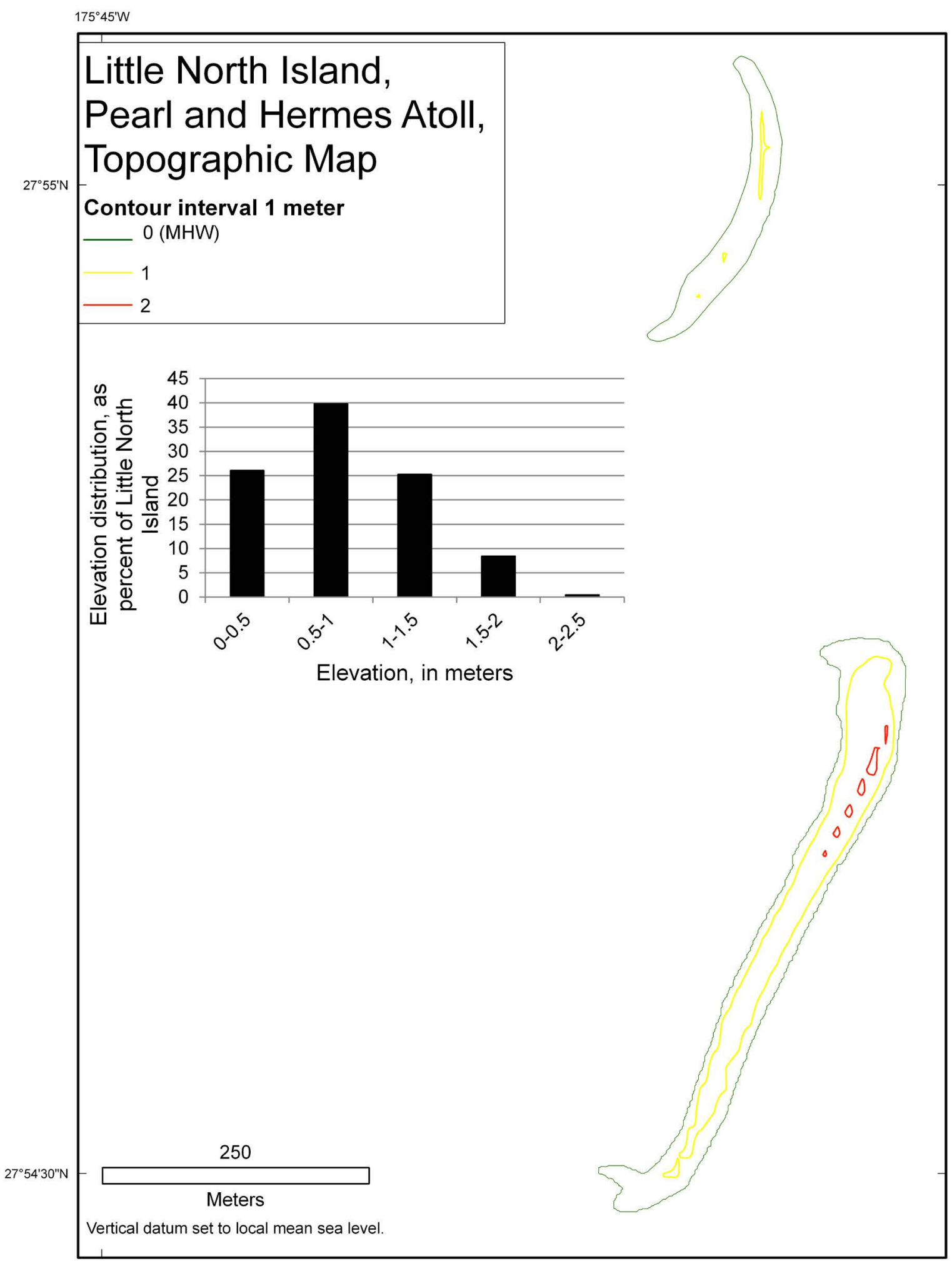

Figure 1.23. Topographic map of Little North Island, Pearl and Hermes Atoll, showing mean high water (MHW) and 1-meter $(\mathrm{m})$ contour lines with a graph of the percentage of land in 0.5-m elevation bins. Elevations were determined from U.S. Geological Survey lidar data (2010) with a vertical root-mean-squared error of $0.12 \mathrm{~m}$. 
$175^{\circ} 49^{\prime} \mathrm{W}$

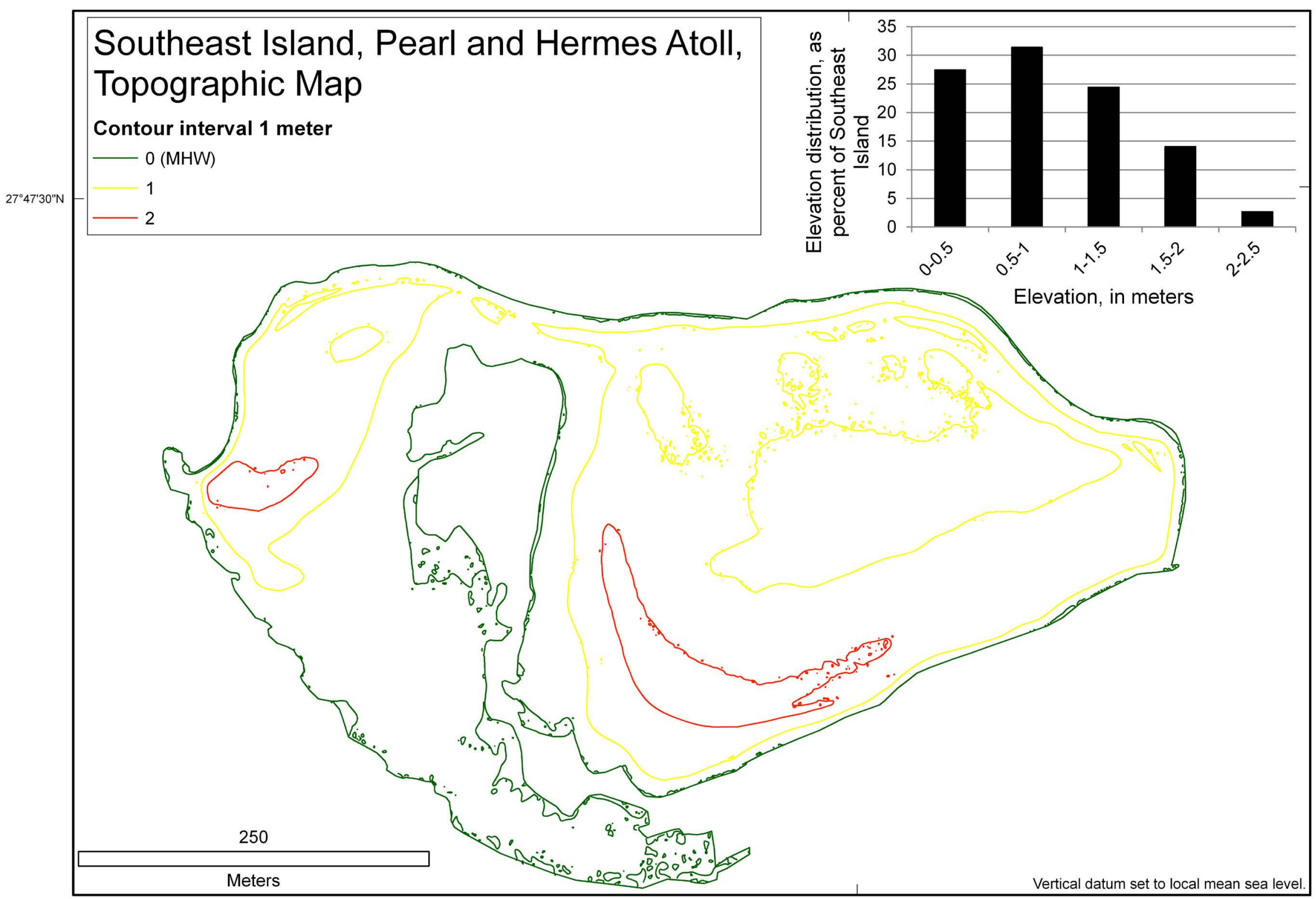

Figure 1.24. Topographic map of Southeast Island, Pearl and Hermes Atoll, showing mean high water (MHW) and 1-meter (m) contour lines with a graph of the percentage of land in 0.5-m elevation bins. Elevations were determined from U.S. Geological Survey lidar data (2010) with a vertical root-mean-squared error of $0.12 \mathrm{~m}$. 


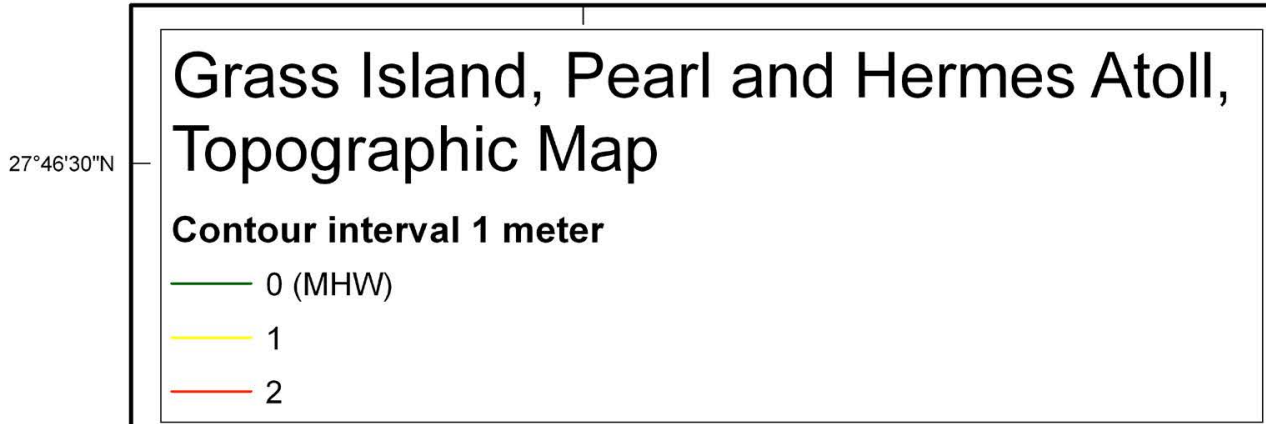

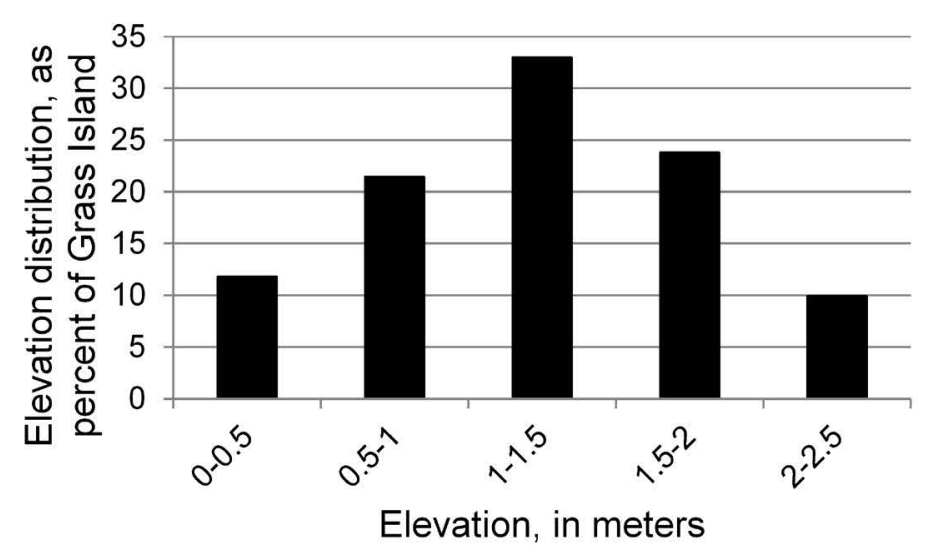

Figure 1.25. Topographic map of Grass Island, Pearl and Hermes Atoll, showing mean high water (MHW) and 1-meter (m) contour lines with a graph of the percentage of land in 0.5-m elevation bins. Elevations were determined from U.S. Geological Survey lidar data (2010) with a vertical root-mean-squared error of $0.12 \mathrm{~m}$. 


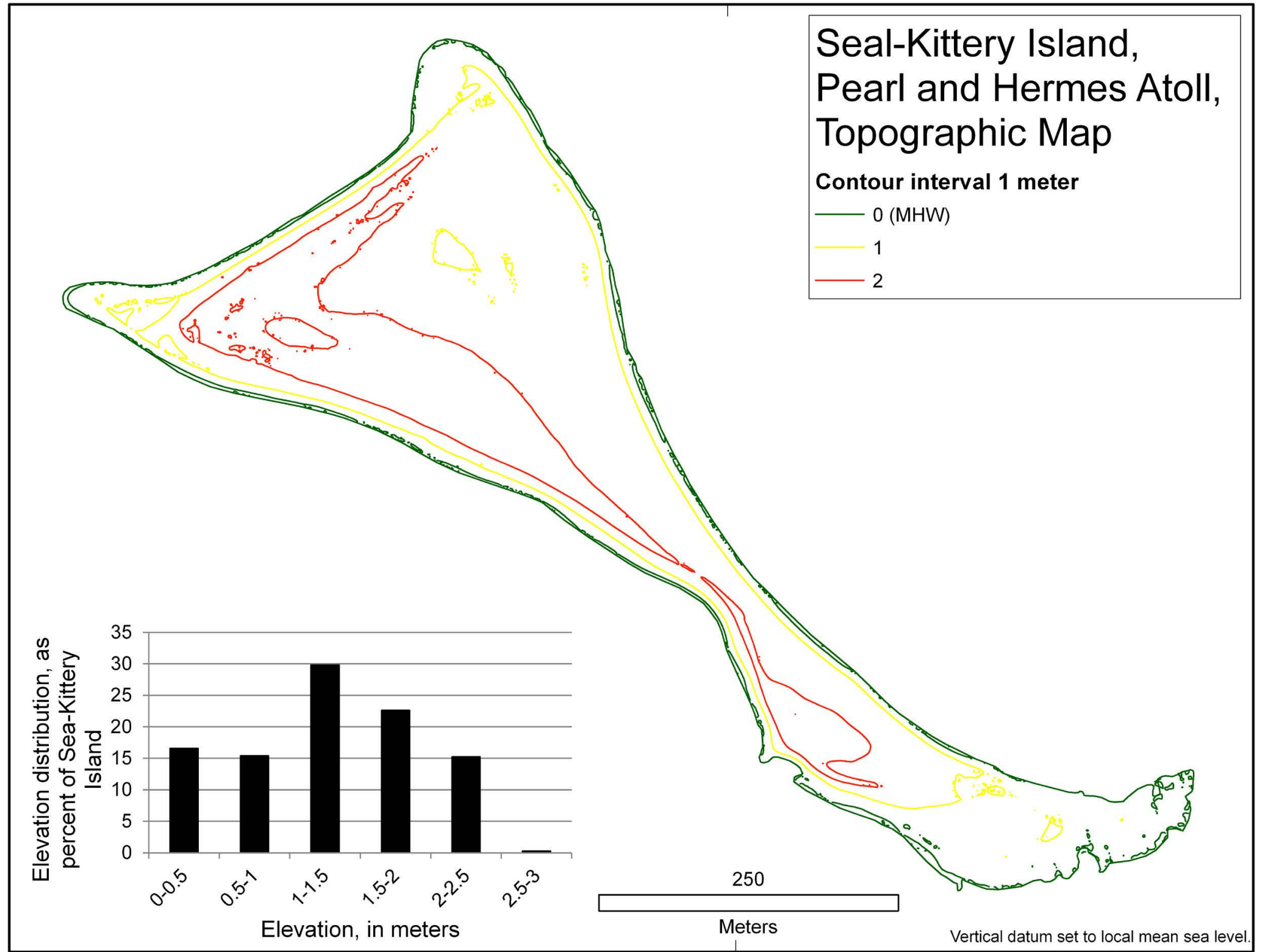

Figure 1.26. Topographic map of Seal-Kittery Island, Pearl and Hermes Atoll, showing mean high water (MHW) and 1-meter (m) contour lines with a graph of the percentage of land in 0.5-m elevation bins. Elevations were determined from U.S. Geological Survey lidar data (2010) with a vertical root-mean-squared error of $0.12 \mathrm{~m}$. 


\section{Pearl and Hermes Atoll Passive Inundation Scenarios}

\section{$0.0 \mathrm{~m}(\mathrm{MHW})$}

+1.0 m sea-level rise inundation area

+2.0 m sea-level rise inundation area
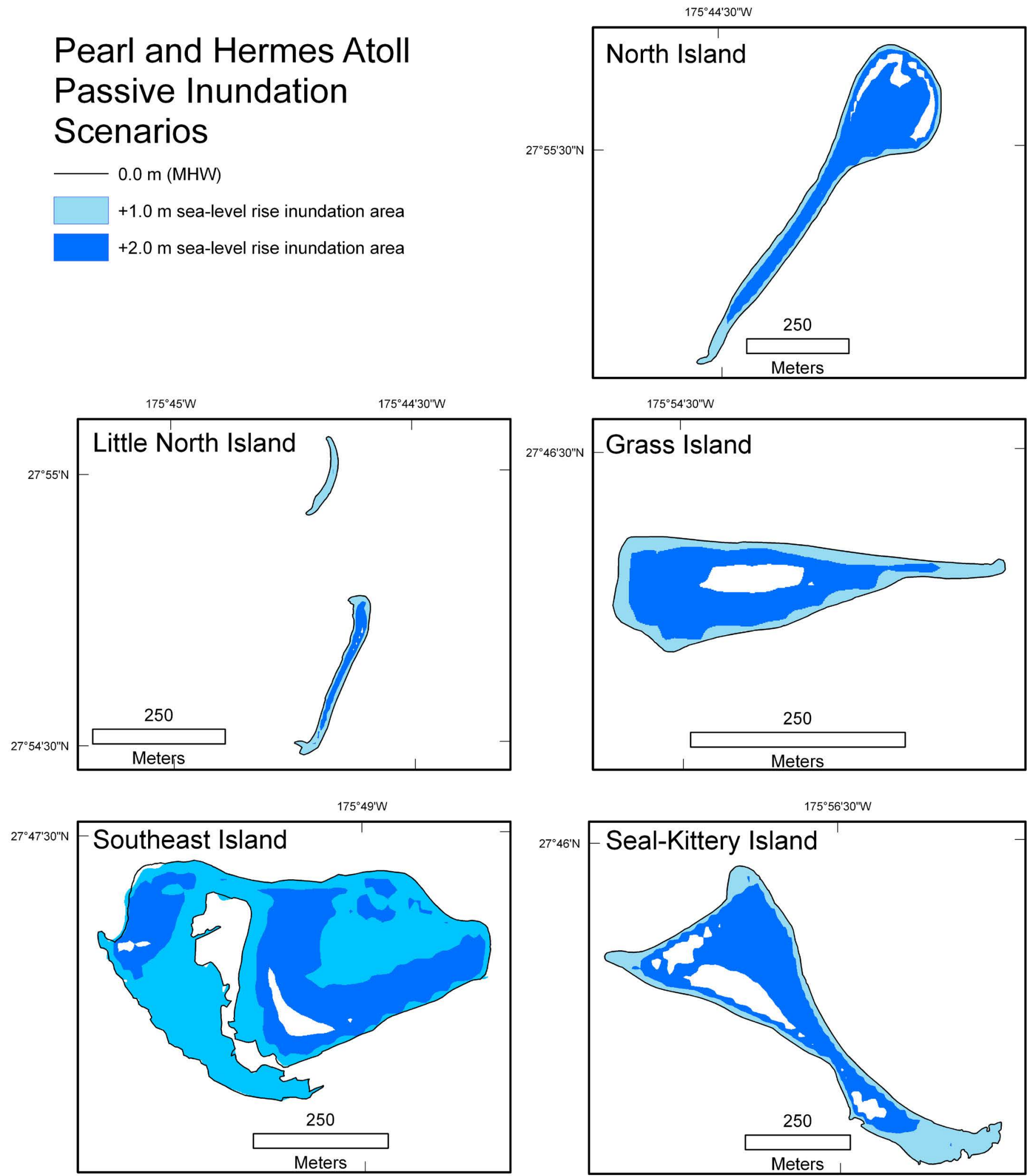

Figure 1.27. Pearl and Hermes Atoll passive inundation scenario maps for five islands at three sea levels: mean high water $(\mathrm{MHW}),+1.0$ and +2.0 meters $(\mathrm{m})$ sea-level rise (SLR). Inundation scenarios are based on U.S. Geological Survey lidar-derived elevations with a root-mean-squared error of $0.12 \mathrm{~m}$. 
Table 1.26. Total potential nesting habitat area and percent change for breeding avifauna at North Island, Pearl and Hermes Atoll, with four sea-level rise (SLR) scenarios $(+0.5,+1.0,+1.5$, and +2.0 meters).

[Areas calculated from land cover classes with Digital Globe QuickBird satellite imagery (October 2007). We assumed percent change from a uniform density across nesting habitat for each species. Abbreviations: $\mathrm{m}$, meters; ha, hectares]

\begin{tabular}{|c|c|c|c|c|c|}
\hline \multirow[b]{2}{*}{ Species } & \multirow{2}{*}{$\begin{array}{c}\text { Area (ha) } \\
0.0 \mathrm{~m}\end{array}$} & \multicolumn{4}{|c|}{ Percent Change from SLR } \\
\hline & & $+0.5 \mathrm{~m}$ & $+1.0 \mathrm{~m}$ & $+1.5 \mathrm{~m}$ & $+2.0 \mathrm{~m}$ \\
\hline Black-footed Albatross & 2.7 & 0.0 & 0.0 & -22.2 & -85.2 \\
\hline Laysan Albatross & 2.7 & 0.0 & 0.0 & -22.2 & -85.2 \\
\hline Red-tailed Tropicbird & 0.2 & 0.0 & 0.0 & -50.0 & -100.0 \\
\hline Masked Booby & 2.7 & 0.0 & 0.0 & -22.2 & -85.2 \\
\hline Brown Booby & 2.7 & 0.0 & 0.0 & -22.2 & -85.2 \\
\hline Sooty Tern & 2.7 & 0.0 & 0.0 & -22.2 & -85.2 \\
\hline Brown Noddy & 2.7 & 0.0 & 0.0 & -22.2 & -85.2 \\
\hline
\end{tabular}

Table 1.27. Total potential nesting habitat area and percent change for breeding avifauna at Little North Island, Pearl and Hermes Atoll, with four sea-level rise (SLR) scenarios $(+0.5,+1.0$, +1.5 , and +2.0 meters).

[Areas calculated from land cover classes with Digital Globe QuickBird satellite imagery (October 2007). We assumed percent change from a uniform density across nesting habitat for each species. Abbreviations: $\mathrm{m}$, meters; ha, hectares]

\begin{tabular}{lcccccc}
\hline & Area (ha) & & \multicolumn{4}{c}{ Percent Change from SLR } \\
\cline { 2 - 4 } \cline { 5 - 7 } Species & $\mathbf{0 . 0 ~}$ & & $\mathbf{+ 0 . 5 ~ \mathbf { ~ }}$ & $\mathbf{+ 1 . 0 \mathrm { m }}$ & $\mathbf{+ 1 . 5 \mathrm { m }}$ & $\mathbf{+ 2 . 0 \mathrm { m }}$ \\
\hline Black-footed Albatross & 0.3 & & 0.0 & 0.0 & 0.0 & -100 \\
Laysan Albatross & 0.3 & & 0.0 & 0.0 & 0.0 & -100 \\
Masked Booby & 0.3 & & 0.0 & 0.0 & 0.0 & -100 \\
\hline
\end{tabular}

Table 1.28. Total potential nesting habitat area and percent change for breeding avifauna at Southeast Island, Pearl and Hermes Atoll, with four sea-level rise (SLR) scenarios $(+0.5,+1.0$, +1.5 , and +2.0 meters).

[Areas calculated from land cover classes with Digital Globe QuickBird satellite imagery (October 2007). We assumed percent change from a uniform density across nesting habitat for each species. Abbreviations: $\mathrm{m}$, meters; ha, hectares]

\begin{tabular}{|c|c|c|c|c|c|}
\hline \multirow[b]{2}{*}{ Species } & \multirow{2}{*}{$\frac{\text { Area (ha) }}{0.0 \mathrm{~m}}$} & \multicolumn{4}{|c|}{ Percent Change from SLR } \\
\hline & & $+0.5 \mathrm{~m}$ & $+1.0 \mathrm{~m}$ & $+1.5 \mathrm{~m}$ & $+2.0 \mathrm{~m}$ \\
\hline Black-footed Albatross & 9.1 & 0.0 & -24.2 & -65.9 & -94.5 \\
\hline Laysan Albatross & 9.1 & 0.0 & -24.2 & -65.9 & -94.5 \\
\hline Bonin Petrel & 8.6 & 0.0 & -23.3 & -65.1 & -94.2 \\
\hline Bulwer's Petrel & 0.5 & 0.0 & -40.0 & -80.0 & -100.0 \\
\hline Wedge-tailed Shearwater & 9.1 & 0.0 & -24.2 & -65.9 & -94.5 \\
\hline Tristram's Storm-petrel & 9.1 & 0.0 & -24.2 & -65.9 & -94.5 \\
\hline Red-tailed Tropicbird & 6.6 & 0.0 & -22.7 & -65.2 & -93.9 \\
\hline Masked Booby & 9.1 & 0.0 & -24.2 & -65.9 & -94.5 \\
\hline Brown Booby & 9.1 & 0.0 & -24.2 & -65.9 & -94.5 \\
\hline Little Tern & 9.1 & 0.0 & -24.2 & -65.9 & -94.5 \\
\hline Gray-backed Tern & 2.5 & 0.0 & -28.0 & -68.0 & -96.0 \\
\hline Sooty Tern & 9.1 & 0.0 & -24.2 & -65.9 & -94.5 \\
\hline Brown Noddy & 9.1 & 0.0 & -24.2 & -65.9 & -94.5 \\
\hline Laysan Finch & 10.5 & -4.8 & -37.1 & -71.4 & -95.2 \\
\hline
\end{tabular}


Table 1.29. Total potential nesting habitat area and percent change for breeding avifauna at Grass Island, Pearl and Hermes Atoll, with four sea-level rise (SLR) scenarios $(+0.5,+1.0,+1.5$, and +2.0 meters).

[Areas calculated from land cover classes with Digital Globe QuickBird satellite imagery (October 2007). We assumed percent change from a uniform density across nesting habitat for each species. Abbreviations: $\mathrm{m}$, meters; ha, hectares]

\begin{tabular}{|c|c|c|c|c|c|}
\hline \multirow[b]{2}{*}{ Species } & \multirow{2}{*}{$\begin{array}{c}\text { Area (ha) } \\
0.0 \mathrm{~m} \\
\end{array}$} & \multicolumn{4}{|c|}{ Percent Change from SLR } \\
\hline & & $+0.5 \mathrm{~m}$ & $+1.0 \mathrm{~m}$ & $+1.5 \mathrm{~m}$ & $+2.0 \mathrm{~m}$ \\
\hline Black-footed Albatross & 1.2 & 0.0 & 0.0 & -8.3 & -75.0 \\
\hline Laysan Albatross & 1.2 & 0.0 & 0.0 & -8.3 & -75.0 \\
\hline Bonin Petrel & 0.5 & 0.0 & 0.0 & 0.0 & -60.0 \\
\hline Wedge-tailed Shearwater & 1.2 & 0.0 & 0.0 & -8.3 & -75.0 \\
\hline Christmas Shearwater & 0.5 & 0.0 & 0.0 & 0.0 & -60.0 \\
\hline Red-tailed Tropicbird & 0.5 & 0.0 & 0.0 & 0.0 & -60.0 \\
\hline Masked Booby & 1.2 & 0.0 & 0.0 & -8.3 & -75.0 \\
\hline Brown Booby & 1.2 & 0.0 & 0.0 & -8.3 & -75.0 \\
\hline Gray-backed Tern & 0.7 & 0.0 & 0.0 & -14.3 & -85.7 \\
\hline Sooty Tern & 1.2 & 0.0 & 0.0 & -8.3 & -75.0 \\
\hline Brown Noddy & 1.2 & 0.0 & 0.0 & -8.3 & -75.0 \\
\hline
\end{tabular}

Table 1.30. Total potential nesting habitat area and percent change for breeding avifauna at Seal-Kittery Island, Pearl and Hermes Atoll, with four sea-level rise (SLR) scenarios $(+0.5,+1.0$, +1.5 , and +2.0 meters).

[Areas calculated from land cover classes with Digital Globe QuickBird satellite imagery (October 2007). We assumed percent change from a uniform density across nesting habitat for each species. Abbreviations: $\mathrm{m}$, meters; ha, hectares; \%, percent]

\begin{tabular}{|c|c|c|c|c|c|}
\hline \multirow[b]{2}{*}{ Species } & \multirow{2}{*}{$\begin{array}{c}\text { Area (ha) } \\
0.0 \mathrm{~m}\end{array}$} & \multicolumn{4}{|c|}{ Percent Change from SLR } \\
\hline & & $+0.5 \mathrm{~m}$ & $+1.0 \mathrm{~m}$ & $+1.5 \mathrm{~m}$ & $+2.0 \mathrm{~m}$ \\
\hline Black-footed Albatross & 7.6 & 0.0 & 0.0 & -35.5 & -72.4 \\
\hline Laysan Albatross & 7.6 & 0.0 & 0.0 & -35.5 & -72.4 \\
\hline Bonin Petrel & 0.5 & 0.0 & 0.0 & -20.0 & -60.0 \\
\hline Wedge-tailed Shearwater & 7.6 & 0.0 & 0.0 & -35.5 & -72.4 \\
\hline Christmas Shearwater & 0.5 & 0.0 & 0.0 & -20.0 & -60.0 \\
\hline Red-tailed Tropicbird & 0.3 & 0.0 & 0.0 & -33.3 & -66.7 \\
\hline Masked Booby & 7.6 & 0.0 & 0.0 & -35.5 & -72.4 \\
\hline Brown Booby & 7.6 & 0.0 & 0.0 & -35.5 & -72.4 \\
\hline Gray-backed Tern & 7.3 & 0.0 & 0.0 & -35.6 & -72.6 \\
\hline Brown Noddy & 7.6 & 0.0 & 0.0 & -35.5 & -72.4 \\
\hline
\end{tabular}




\section{Lisianski Island}

\section{Island Information}

Lisianski Island measured 147.1 ha in area (table 1.9, fig. 1.28). Five land cover classes were identified on Lisianski: tree/shrub including Chenopodium oahuense, Pisonia grandis, Scaevola taccada, and Tournefortia argentea; grass/herbaceous cover including Cenchrus echinatus, Eragrostis variabilis, Lepturus repens, and Solanum americanum; vine/ground cover including Boerhavia repens, Ipomoea indica, Ipomoea pes-caprae, Nama sandwicensis, Portulaca lutea, Sicyos pachycarpus, and Tribulus cistoides; bare ground; and beach (table 1.31, fig. 1.29). The dominant land cover was grass/ herbaceous cover at 96.2 ha (65 percent; table 1.31).

\section{Sea-Level Rise}

The maximum elevation of Lisianski Island was $7.6 \mathrm{~m}$ with a mean of $3.8 \mathrm{~m}$ (SD 1.3, RMSE 0.18; table 1.9). Of Lisianski's total land area, 5 percent was below $2.0 \mathrm{~m}, 59$ percent was below $4.0 \mathrm{~m}$, and 93 percent was below $6.0 \mathrm{~m}$ elevation (fig. 1.30). Total area loss of 2 percent at $+1.0 \mathrm{~m}$ SLR and 5 percent at $+2.0 \mathrm{~m}$ SLR was expected for Lisianski Island with the passive inundation model (table 1.31, fig. 1.31). Vegetated area loss was 0.2 percent at $+1.0 \mathrm{~m}$ SLR and 0.3 percent at $+2.0 \mathrm{~m}$ SLR (table 1.31 ). Only beach area was reduced by more than 3 percent due to inundation at $+2.0 \mathrm{~m}$ SLR (table 1.31). Less than 3 percent of avian breeding habitat was expected to be lost for any species at or below $+2.0 \mathrm{~m}$ SLR (table 1.32).

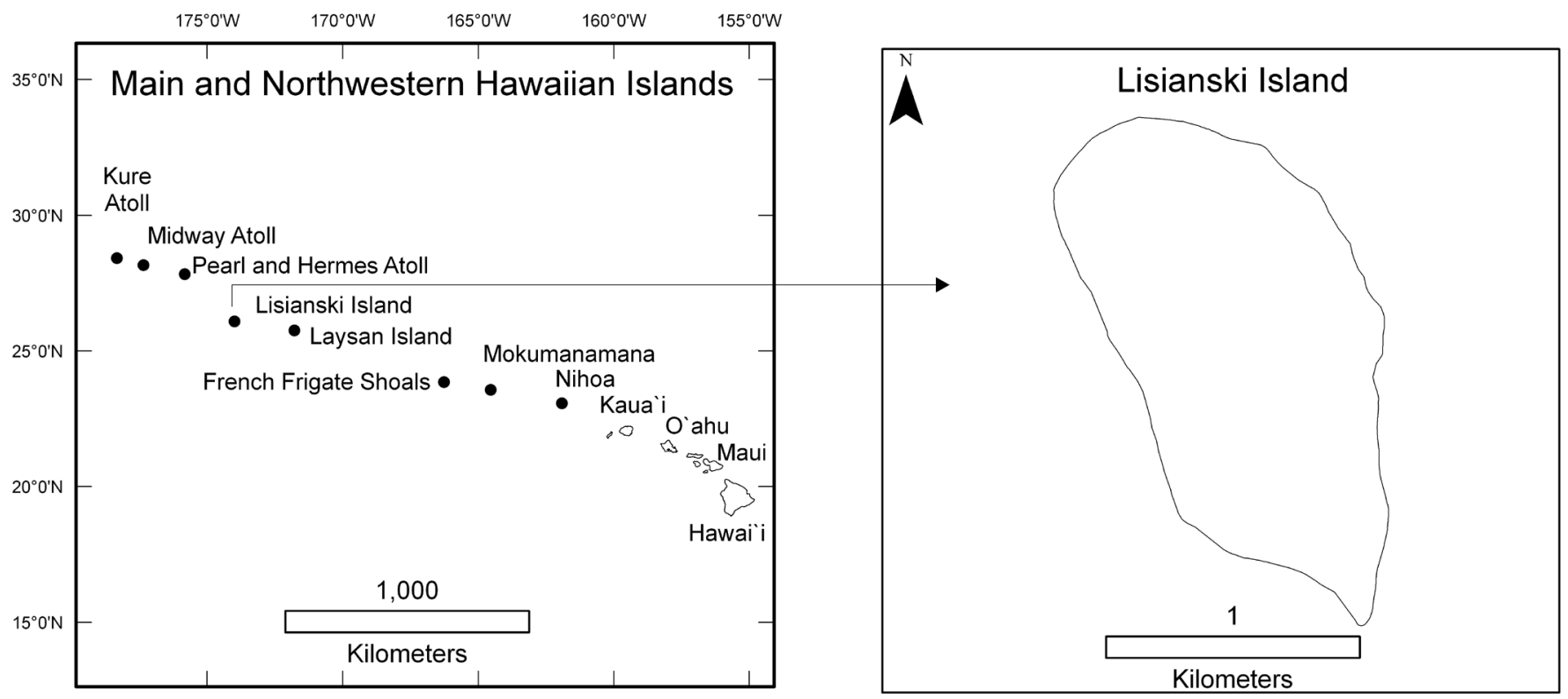

Figure 1.28. Maps showing the location of Lisianski Island, a low sandy island situated between Pearl and Hermes Atoll to the northwest and Laysan Island to the southeast.

Table 1.31. Total land area and percent change for each of five land cover types at Lisianski Island with four sea-level rise (SLR) scenarios $(+0.5,+1.0,+1.5$, and +2.0 meters).

[Areas calculated from Digital Globe QuickBird satellite imagery (January 2008). Abbreviations: $m$, meters; ha, hectares; \%, percent]

\begin{tabular}{|c|c|c|c|c|c|c|c|c|c|}
\hline \multirow[b]{3}{*}{ Land cover } & \multirow{3}{*}{$\frac{0.0 \mathrm{~m}}{\text { Area (ha) }}$} & \multicolumn{8}{|c|}{ Change from SLR } \\
\hline & & \multicolumn{2}{|c|}{$+0.5 \mathrm{~m}$} & \multicolumn{2}{|c|}{$+1.0 \mathrm{~m}$} & \multicolumn{2}{|c|}{$+1.5 \mathrm{~m}$} & \multicolumn{2}{|c|}{$+2.0 \mathrm{~m}$} \\
\hline & & Area (ha) & $\%$ change & Area (ha) & $\%$ change & Area (ha) & $\%$ change & Area (ha) & $\%$ change \\
\hline Tree/shrub & 10.6 & 10.6 & 0.0 & 10.6 & 0.0 & 10.6 & 0.0 & 10.6 & 0.0 \\
\hline Grass/herbaceous cover & r 96.2 & 96.1 & -0.1 & 96.1 & -0.1 & 96.1 & -0.1 & 95.9 & -0.3 \\
\hline Vine/ground cover & 17.2 & 17.1 & -0.6 & 17.1 & -0.6 & 17.1 & -0.6 & 17.1 & -0.6 \\
\hline Bare ground & 11.9 & 11.9 & 0.0 & 11.9 & 0.0 & 11.8 & -0.8 & 11.6 & -2.5 \\
\hline Total island size & 147.1 & 145.1 & -1.4 & 143.7 & -2.3 & 142.2 & -3.3 & 140.4 & -4.6 \\
\hline
\end{tabular}




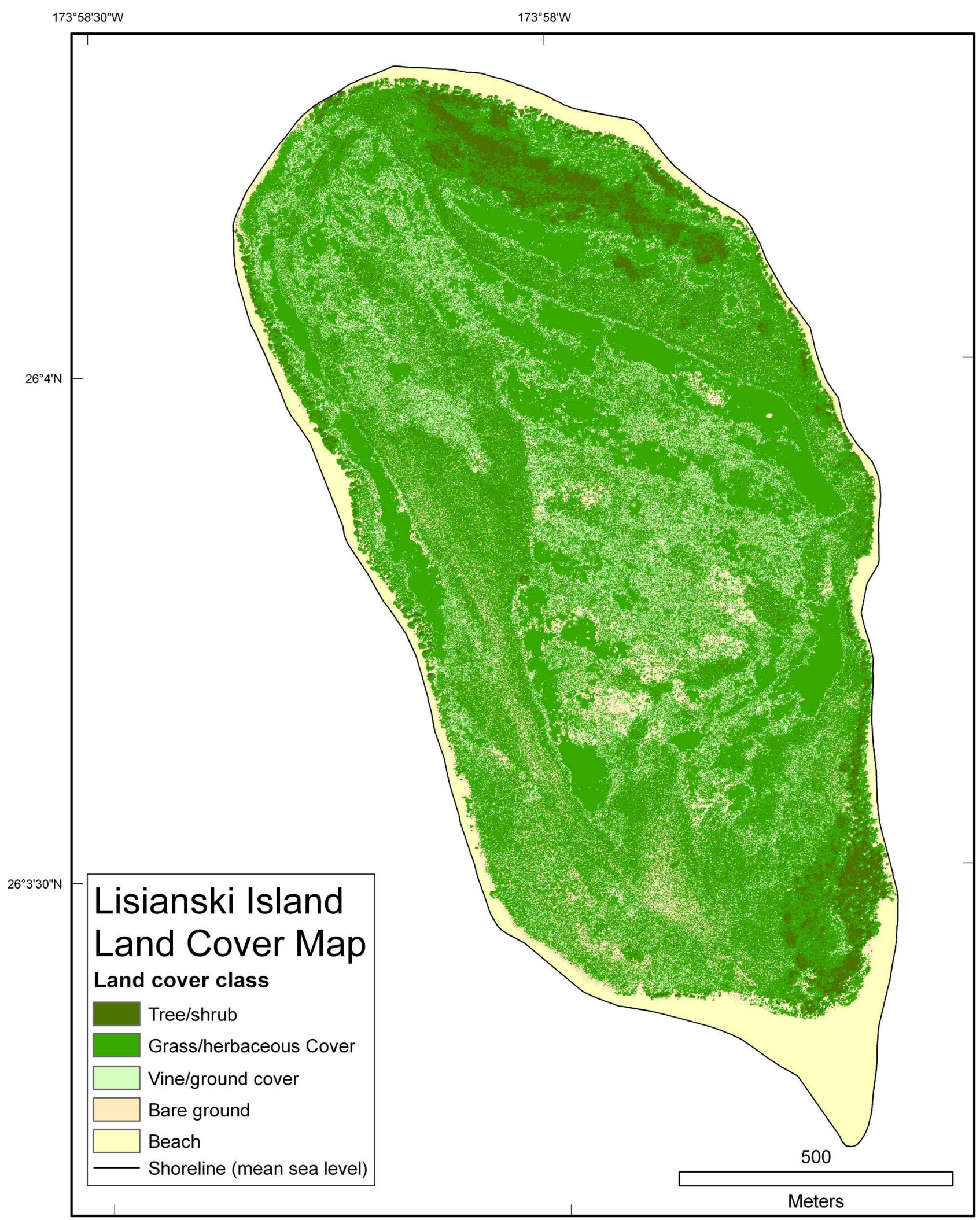

Figure 1.29. Lisianski Island land cover classification map developed using primarily unsupervised classification methods from Digital Globe QuickBird satellite imagery (January 2008). Additional details on land cover are given in table 1.31 . 


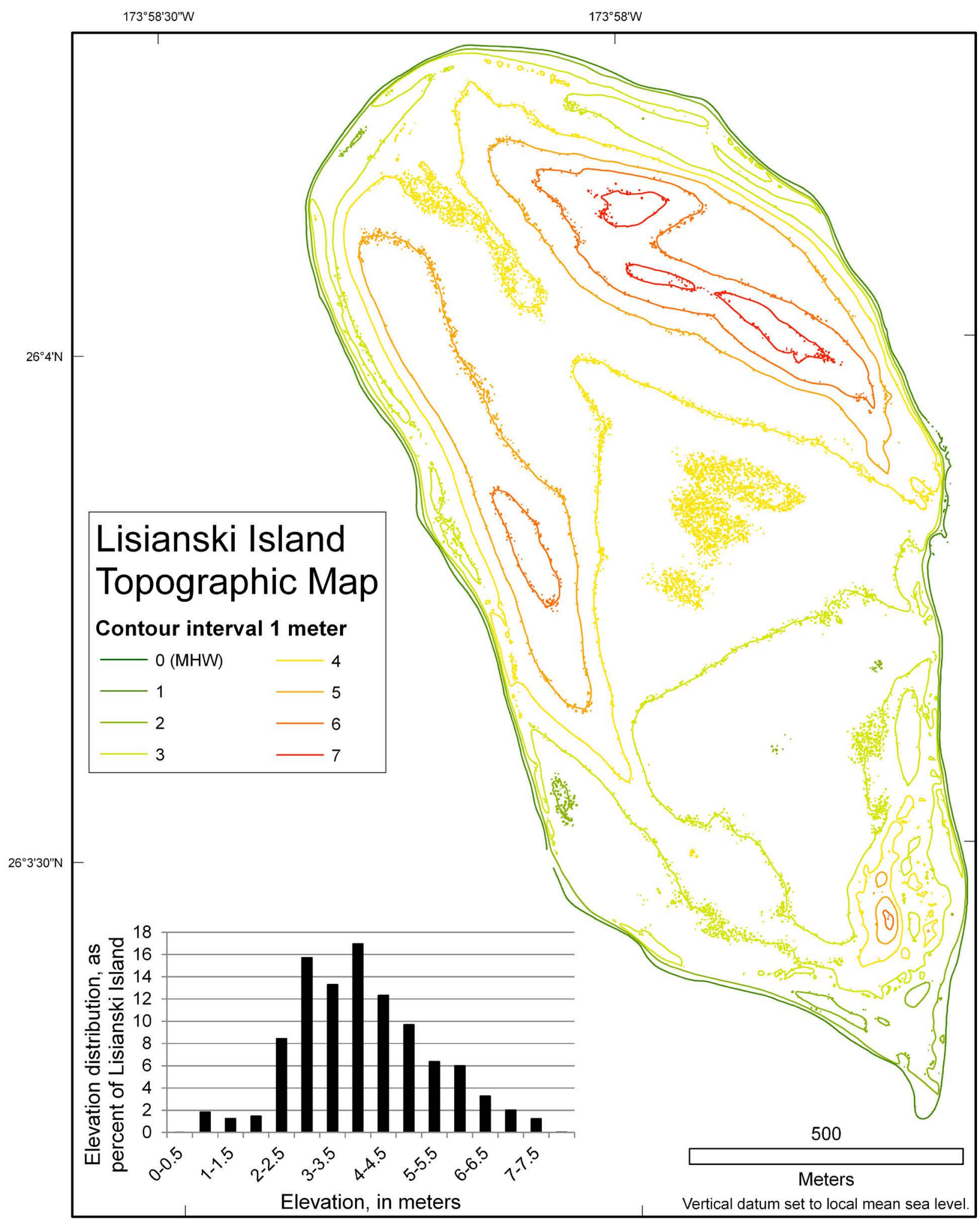

Figure 1.30. Topographic map of Lisianski Island showing mean high water (MHW) and 1-meter (m) contour lines with a graph of the percentage of land in $0.5-\mathrm{m}$ elevation bins. Elevations were determined from U.S. Geological Survey lidar data (2010) with a vertical root-mean-squared error of $0.18 \mathrm{~m}$. 


\section{Lisianski Island} Passive Inundation Scenarios

Inundation area

\section{Elevation (m)}
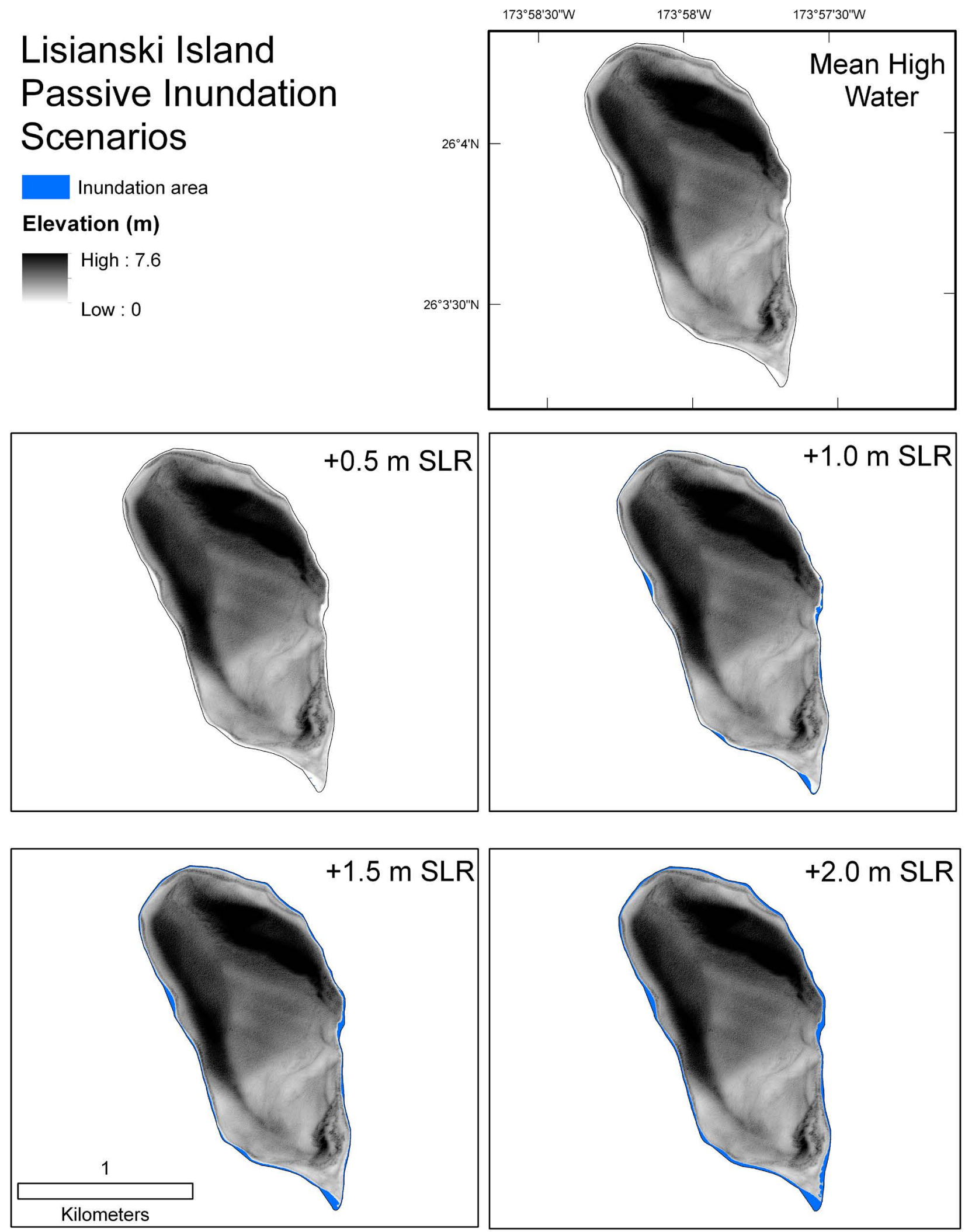

Figure 1.31. Lisianski Island passive inundation scenario maps for five sea levels: mean high water through +2.0 meters $(\mathrm{m})$ sea-level rise (SLR) at 0.5-m increments. Inundation scenarios are based on U.S. Geological Survey lidar-derived elevations with a root-mean-squared error of $0.18 \mathrm{~m}$. 
Table 1.32. Total potential nesting habitat area and percent change for breeding avifauna at Lisianski Island with four sea-level rise (SLR) scenarios $(+0.5,+1.0,+1.5$, and +2.0 meters).

[Areas calculated from land cover classes with Digital Globe QuickBird satellite imagery (January 2008). We based percent change on a uniform density across nesting habitat for each species. Abbreviations: $\mathrm{m}$, meters; ha, hectares]

\begin{tabular}{|c|c|c|c|c|c|}
\hline \multirow[b]{2}{*}{ Species } & \multirow{2}{*}{$\begin{array}{c}\text { Area (ha) } \\
0.0 \mathrm{~m}\end{array}$} & \multicolumn{4}{|c|}{ Percent Change from SLR } \\
\hline & & $+0.5 \mathrm{~m}$ & $+1.0 \mathrm{~m}$ & $+1.5 \mathrm{~m}$ & $+2.0 \mathrm{~m}$ \\
\hline Black-footed Albatross & 125.3 & -0.2 & -0.2 & -0.2 & -0.6 \\
\hline Laysan Albatross & 125.3 & -0.2 & -0.2 & -0.2 & -0.6 \\
\hline Bonin Petrel & 124.0 & -0.2 & -0.2 & -0.2 & -0.3 \\
\hline Bulwer's Petrel & 11.9 & 0.0 & 0.0 & -0.8 & -2.5 \\
\hline Wedge-tailed Shearwater & 135.9 & -0.1 & -0.1 & -0.2 & -0.5 \\
\hline Christmas Shearwater & 124.0 & -0.2 & -0.2 & -0.2 & -0.3 \\
\hline Red-tailed Tropicbird & 106.8 & -0.1 & -0.1 & -0.1 & -0.3 \\
\hline Masked Booby & 125.3 & -0.2 & -0.2 & -0.2 & -0.6 \\
\hline Brown Booby & 125.3 & -0.2 & -0.2 & -0.2 & -0.6 \\
\hline Red-footed Booby & 10.6 & 0.0 & 0.0 & 0.0 & 0.0 \\
\hline Great Frigatebird & 10.6 & 0.0 & 0.0 & 0.0 & 0.0 \\
\hline Gray-backed Tern & 29.1 & -0.3 & -0.3 & -0.7 & -1.4 \\
\hline Sooty Tern & 125.3 & -0.2 & -0.2 & -0.2 & -0.6 \\
\hline Brown Noddy & 135.9 & -0.1 & -0.1 & -0.2 & -0.5 \\
\hline Black Noddy & 10.6 & 0.0 & 0.0 & 0.0 & 0.0 \\
\hline White Tern & 10.6 & 0.0 & 0.0 & 0.0 & 0.0 \\
\hline
\end{tabular}




\section{Laysan Island}

\section{Island Information}

The total area of Laysan Island was 413.6 ha, of which 339.4 ha were terrestrial; the lake and mudflat area accounted for the remaining 74.2 ha (table 1.9, fig. 1.32). Eleven land cover classes were identified on Laysan: tree/shrub including Scaevola taccada; mixed shrub; grass/herbaceous cover including Eragrostis variabilis and Fimbristylis cymosa; vine/ ground cover including Boerhavia repens, Ipomoea pescaprae, Sicyos ssp., and Tribulus cistoides; wetland vegetation including Cyperus laevigatus, Heliotropium currasavicum, and Sesuvium portulacastrum; bare ground; hard pan; beach; wetland (unvegetated); wetland (standing water); and human structure (fig. 1.33, see table 2.3). Four additional speciesspecific land cover classes based on distribution data included: Casuarina equisetifolia, Cocos nucifera, Pluchea indica, and Tournefortia argentea. The lake zone at Laysan comprised two land cover classes: (1) unvegetated wetland area including mudflats and (2) standing water. Though this area varies in size seasonally, from visual inspection of the May 18, 2010, WorldView-2 image (Digital Globe, 2010), the lake zone was made up of 34.2 ha of unvegetated area and 40.0 ha of standing water. The dominant land cover at Laysan Island was bare ground (129.3 ha; table 2.3).

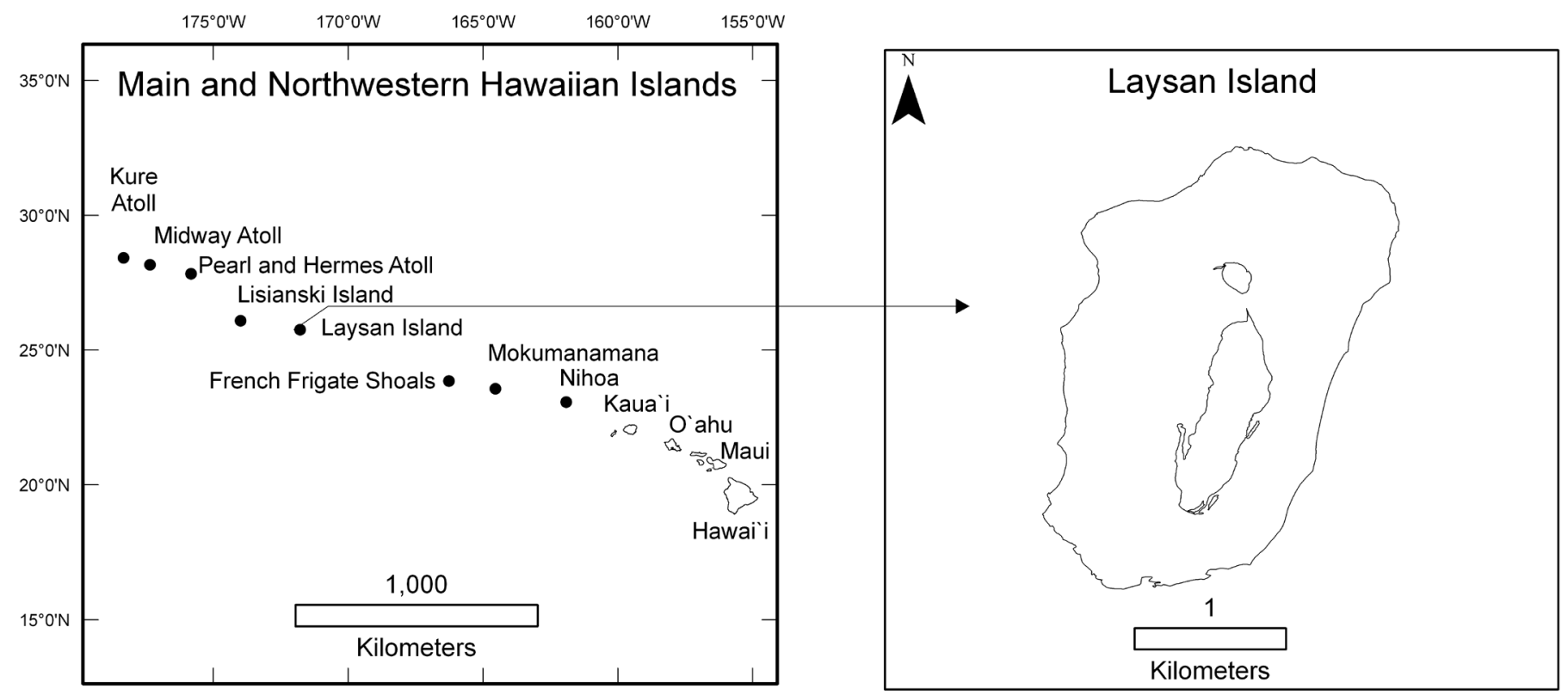

Figure 1.32. Maps showing location of Laysan Island, a low carbonate island situated between Lisianski Island to the northwest and French Frigate Shoals to the southeast. 


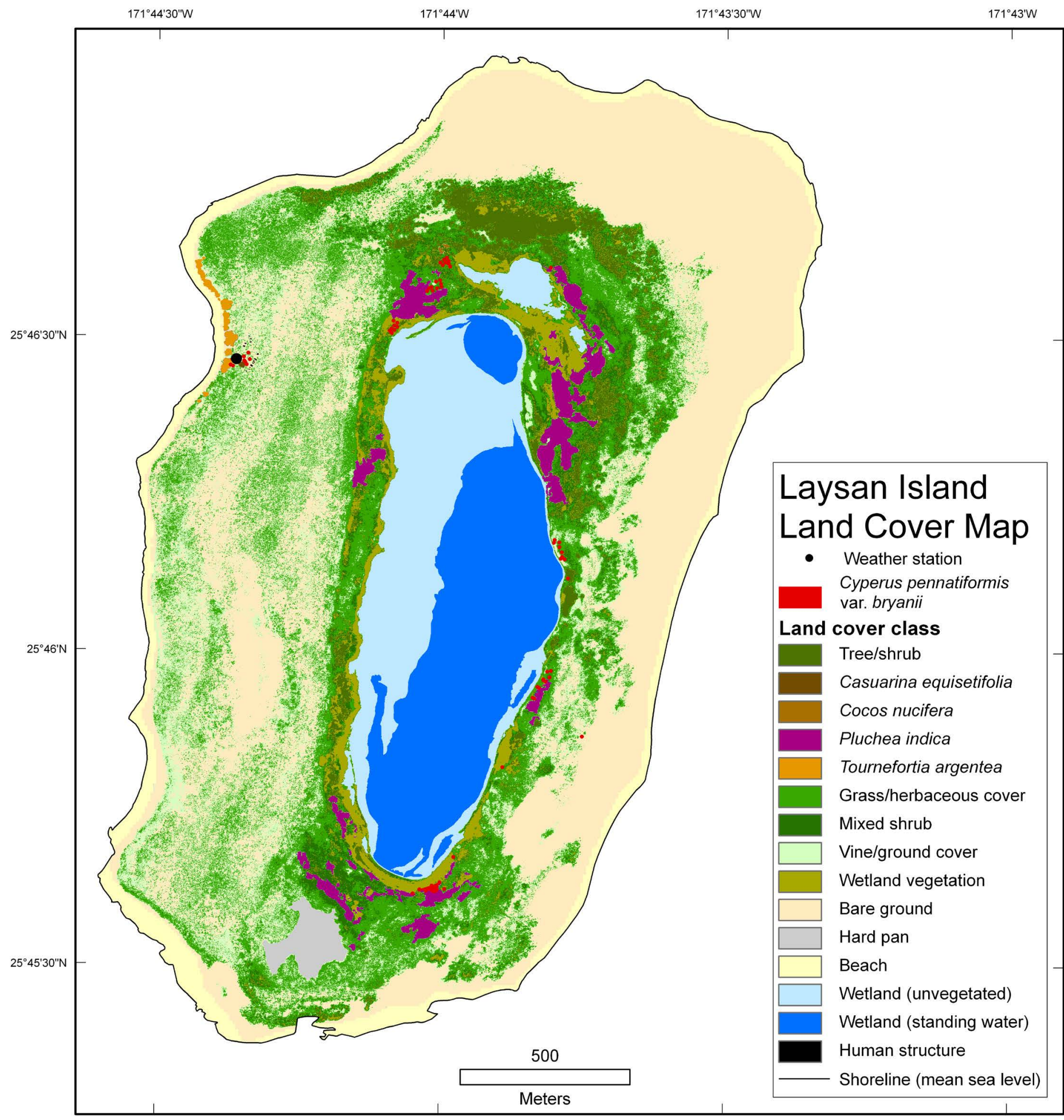

Figure 1.33. Laysan Island land cover classification map developed using primarily unsupervised classification methods from Digital Globe WorldView-2 satellite imagery (May 2010). Species-specific plant delineations mapped from U.S. Fish and Wildlife Service (unpub. data). See chap. 2 for additional details on land cover and species composition. 


\section{Sea-Level Rise}

The maximum elevation of Laysan Island was $10.7 \mathrm{~m}$ with a mean of $4.3 \mathrm{~m}$ (SD 2.3, RMSE 0.09; table 1.9) excluding the lake zone. The mean elevation of Laysan Island including the lake zone was $3.5 \mathrm{~m}$ (SD 2.6). Of the terrestrial area of Laysan Island (excludes lake zone), 31 percent was below $2.0 \mathrm{~m}$ elevation and 50 percent below $3.5 \mathrm{~m}$ (fig. 1.34). Scenarios of passive inundation that did not incorporate rising groundwater showed total area losses of 1 percent at $+1.0 \mathrm{~m}$ SLR and 4 percent at
$+2.0 \mathrm{~m}$ SLR (fig. 1.35, table 1.9). In contrast, total area loss with passive inundation and a concurrent groundwater rise was expected to be 7 percent at $+1.0 \mathrm{~m}$ SLR and 24 percent at $+2.0 \mathrm{~m}$ SLR (fig. 1.36, table 1.9).

Models without rising lake levels indicated that less than 4 percent of breeding habitat would be lost across all SLR scenarios (table 1.33a). If lake levels were to rise with SLR, greater losses in breeding habitat would occur for all bird species, especially shrub-nesting species with as much as 63 percent habitat loss at $+2.0 \mathrm{~m}$ SLR (table 1.33b).

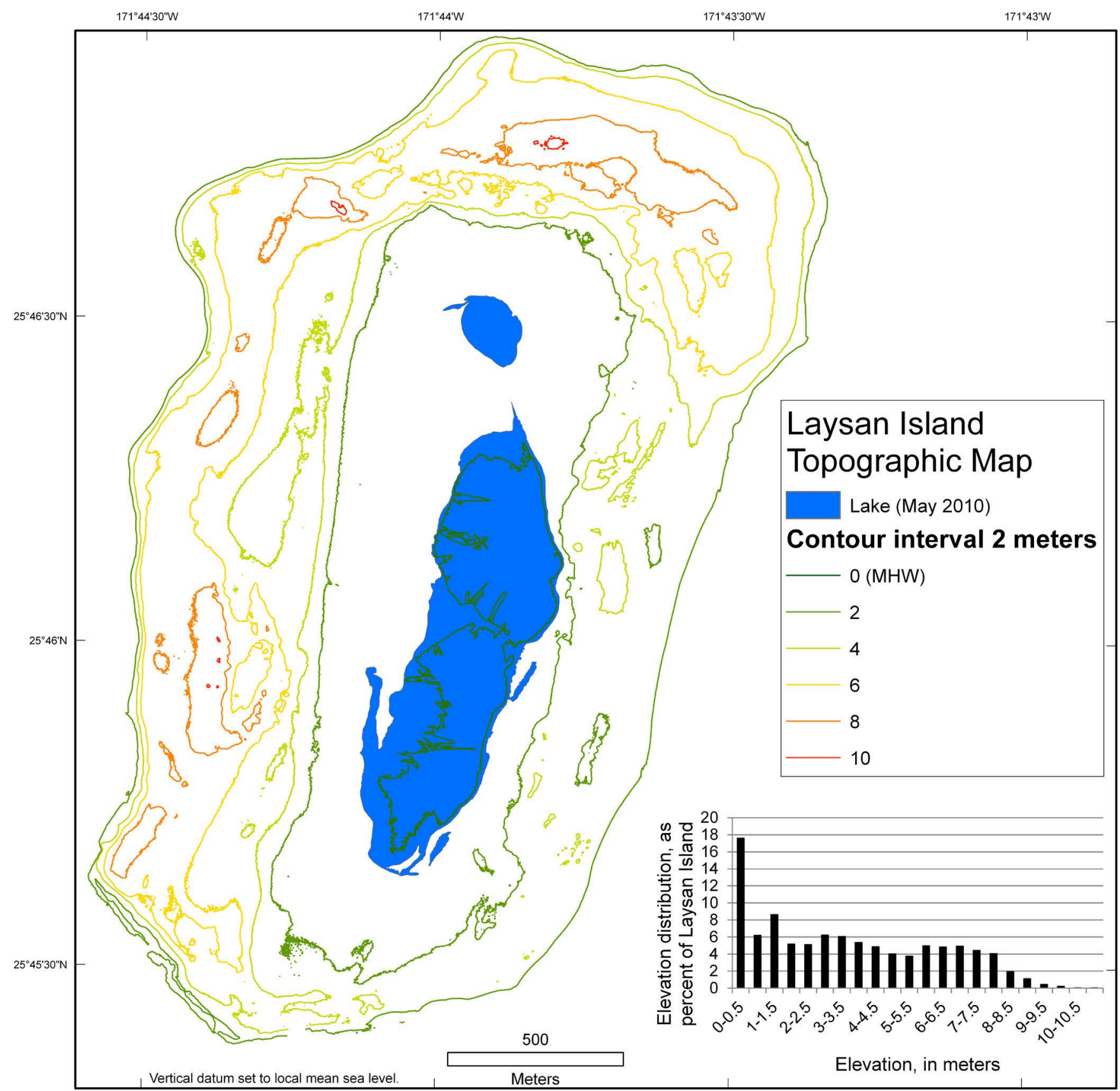

Figure 1.34. Topographic map of Laysan Island showing mean high water (MHW) and 2-meter $(\mathrm{m})$ contour lines with a graph of the percentage of land in $0.5-\mathrm{m}$ elevation bins. Elevations were determined from U.S. Geological Survey lidar data (2010) with a vertical root-mean-squared error of $0.09 \mathrm{~m}$. 


\section{Laysan Island} Passive Inundation Scenarios

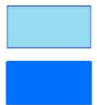

Lake (May 2010)

Inundation area

Elevation ( $\mathrm{m}$ )

High : 10.7

Low : -0.5

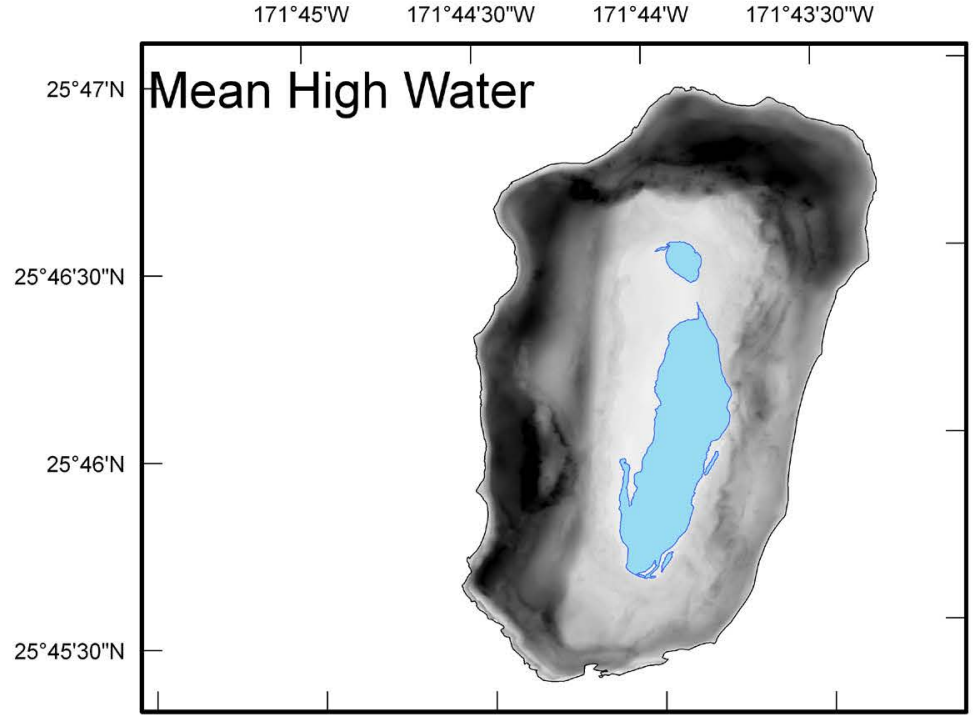

$$
+0.5 \mathrm{~m} \mathrm{SLR}
$$

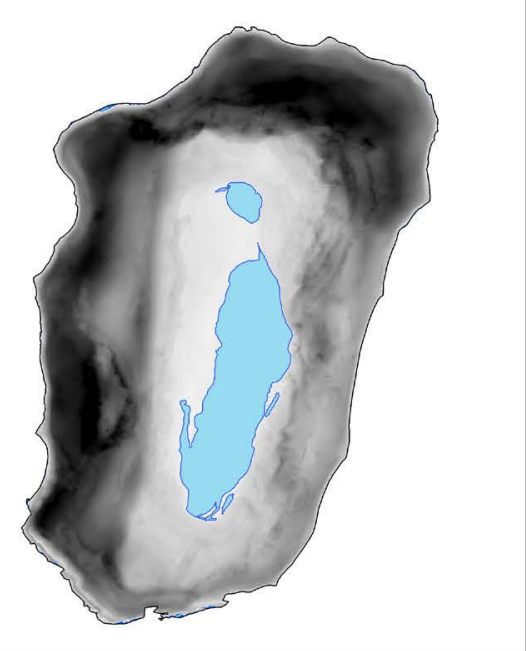

\section{+1.0 m SLR}
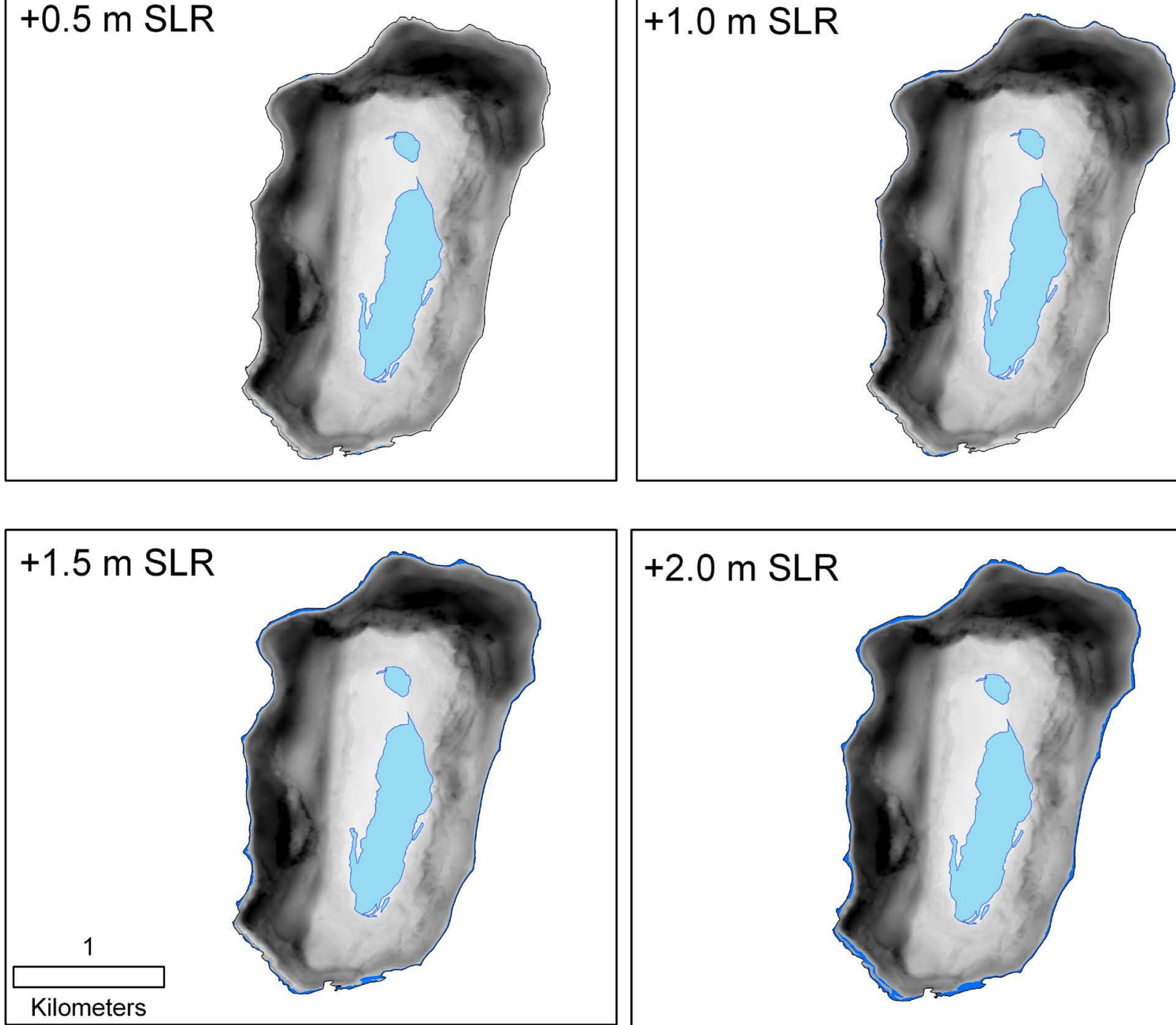

\section{+2.0 m SLR}

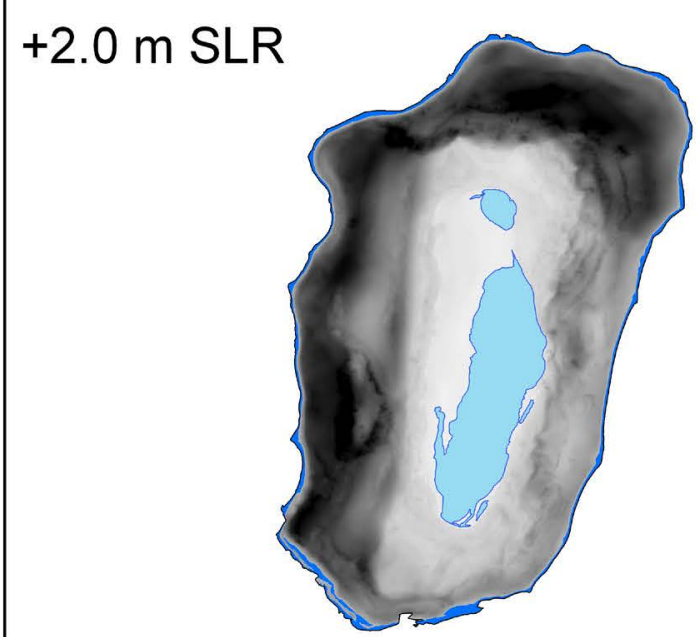

Figure 1.35. Laysan Island passive inundation scenario maps for five sea levels: mean high water through $+2.0 \mathrm{~m}$ (meters) sea-level rise (SLR) at 0.5-m increments, assuming no change in groundwater lake levels. Inundation scenarios are based on U.S. Geological Survey lidar-derived digital elevations with a root-mean-squared error of $0.09 \mathrm{~m}$. 


\section{Laysan Island Passive Inundation Scenarios with Groundwater Rise}

Lake (May 2010)

Inundation area

Elevation (m)

High : 10.7

Low : -0.5
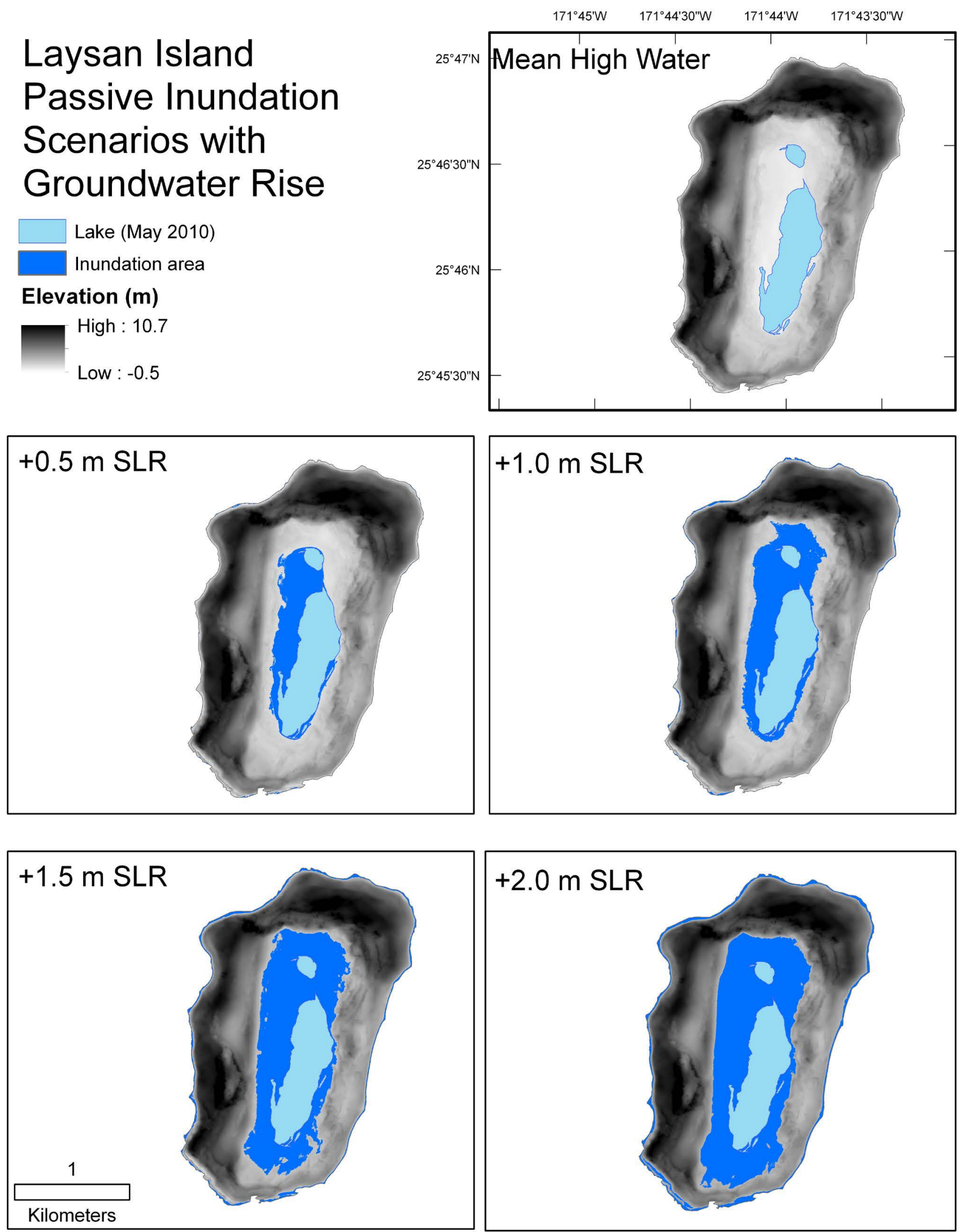

Figure 1.36. Laysan Island passive inundation scenario maps for five sea levels: mean high water through +2.0 meters $(\mathrm{m})$ sea-level rise (SLR) at 0.5-m increments, assuming groundwater rise in lake levels. Inundation scenarios are based on U.S. Geological Survey lidar-derived elevations with a root-mean-squared error of $0.09 \mathrm{~m}$. 
Table 1.33. Total potential nesting habitat area and percent change for breeding avifauna at Laysan Island with four sea-level rise (SLR) scenarios $(+0.5,+1.0,+1.5$, and +2.0 meters) using (a) current lake levels and (b) lake levels rising with groundwater and SLR.

[Areas calculated from land cover classes with Digital Globe WorldView-2 satellite imagery (May 2010). We computed percent change based on uniform species density across nesting habitat for each species. (a) Laysan Island, assuming current lake levels; (b) Laysan Island, assuming rising lake levels with sea-level rise. Abbreviations: $\mathrm{m}$, meters; ha, hectares]

a.

\begin{tabular}{|c|c|c|c|c|c|}
\hline \multirow[b]{2}{*}{ Species } & \multirow{2}{*}{$\begin{array}{c}\text { Area (ha) } \\
0.0 \mathrm{~m}\end{array}$} & \multicolumn{4}{|c|}{ Percent Change from SLR } \\
\hline & & $+0.5 \mathrm{~m}$ & $+1.0 \mathrm{~m}$ & $+1.5 \mathrm{~m}$ & $+2.0 \mathrm{~m}$ \\
\hline Black-footed Albatross & 280.1 & 0.0 & -1.0 & -2.4 & -3.8 \\
\hline Laysan Albatross & 283.5 & 0.0 & -1.0 & -2.4 & -3.7 \\
\hline Bonin Petrel & 165.4 & 0.0 & -1.0 & -2.4 & -3.8 \\
\hline Bulwer's Petrel & 133.6 & 0.0 & -1.0 & -2.4 & -3.7 \\
\hline Wedge-tailed Shearwater & 299.0 & 0.0 & -1.0 & -2.4 & -3.8 \\
\hline Christmas Shearwater & 165.4 & 0.0 & -1.0 & -2.4 & -3.8 \\
\hline Tristram's Storm-petrel & 299.0 & 0.0 & -1.0 & -2.4 & -3.8 \\
\hline Red-tailed Tropicbird & 111.2 & 0.0 & -1.1 & -2.4 & -3.9 \\
\hline Masked Booby & 266.4 & 0.0 & -1.0 & -2.4 & -3.7 \\
\hline Brown Booby & 263.0 & 0.0 & -1.0 & -2.4 & -3.8 \\
\hline Red-footed Booby & 36.0 & 0.0 & -1.1 & -2.2 & -3.9 \\
\hline Great Frigatebird & 36.0 & 0.0 & -1.1 & -2.2 & -3.9 \\
\hline Gray-backed Tern & 191.2 & 0.0 & -1.0 & -2.4 & -3.7 \\
\hline Sooty Tern & 263.0 & 0.0 & -1.0 & -2.4 & -3.8 \\
\hline Brown Noddy & 299.0 & 0.0 & -1.0 & -2.4 & -3.8 \\
\hline Black Noddy & 36.0 & 0.0 & -1.1 & -2.2 & -3.9 \\
\hline White Tern & 28.0 & 0.0 & -1.1 & -2.1 & -3.9 \\
\hline Laysan Teal & 165.4 & 0.0 & -1.0 & -2.4 & -3.8 \\
\hline Laysan Finch & 165.4 & 0.0 & -1.0 & -2.4 & -3.8 \\
\hline Nihoa Millerbird* & 165.4 & 0.0 & -1.0 & -2.4 & -3.8 \\
\hline
\end{tabular}

*Expected habitat use from newly translocated population to Lavsan Island. 
Table 1.33. Total potential nesting habitat area and percent change for breeding avifauna at Laysan Island with four sea-level rise (SLR) scenarios $(+0.5,+1.0,+1.5$, and +2.0 meters) using (a) current lake levels and (b) lake levels rising with groundwater and SLR.-Continued [Areas calculated from land cover classes with Digital Globe WorldView-2 satellite imagery (May 2010). We computed percent change based on uniform species density across nesting habitat for each species. (a) Laysan Island, assuming current lake levels; (b) Laysan Island, assuming rising lake levels with sea-level rise. Abbreviations: $m$, meters; ha, hectares] b.

\begin{tabular}{|c|c|c|c|c|c|}
\hline \multirow[b]{2}{*}{ Species } & \multirow{2}{*}{$\begin{array}{c}\text { Area (ha) } \\
0.0 \mathrm{~m} \\
\end{array}$} & \multicolumn{4}{|c|}{ Percent Change from SLR } \\
\hline & & $+0.5 \mathrm{~m}$ & $+1.0 \mathrm{~m}$ & $+1.5 \mathrm{~m}$ & $+2.0 \mathrm{~m}$ \\
\hline Black-footed Albatross & 280.1 & -0.1 & -2.5 & -9.7 & -14.2 \\
\hline Laysan Albatross & 283.5 & -0.1 & -2.5 & -9.9 & -15.0 \\
\hline Bonin Petrel & 165.4 & -0.2 & -6.0 & -23.2 & -30.8 \\
\hline Bulwer's Petrel & 133.6 & 0.0 & -0.1 & -0.3 & -0.9 \\
\hline Wedge-tailed Shearwater & 299.0 & -0.1 & -3.4 & -13.0 & -17.4 \\
\hline Christmas Shearwater & 165.4 & -0.2 & -6.0 & -23.2 & -30.8 \\
\hline Tristram's Storm-petrel & 299.0 & -0.1 & -3.4 & -13.0 & -17.4 \\
\hline Red-tailed Tropicbird & 111.2 & -0.3 & -8.5 & -32.9 & -41.9 \\
\hline Masked Booby & 266.4 & -0.1 & -1.8 & -7.2 & -12.0 \\
\hline Brown Booby & 263.0 & -0.1 & -1.7 & -7.0 & -11.2 \\
\hline Red-footed Booby & 36.0 & -0.3 & -15.3 & -56.9 & -63.1 \\
\hline Great Frigatebird & 36.0 & -0.3 & -15.3 & -56.9 & -63.1 \\
\hline Gray-backed Tern & 191.2 & -0.1 & -0.5 & -1.6 & -4.2 \\
\hline Sooty Tern & 263.0 & -0.1 & -1.7 & -7.0 & -11.2 \\
\hline Brown Noddy & 299.0 & -0.1 & -3.4 & -13.0 & -17.4 \\
\hline Black Noddy & 36.0 & -0.3 & -15.3 & -56.9 & -63.1 \\
\hline White Tern & 28.0 & -0.4 & -14.3 & -45.0 & -52.5 \\
\hline Laysan Teal & 165.4 & -0.2 & -6.0 & -23.2 & -30.8 \\
\hline Laysan Finch & 165.4 & -0.2 & -6.0 & -23.2 & -30.8 \\
\hline Nihoa Millerbird* & 165.4 & -0.2 & -6.0 & -23.2 & -30.8 \\
\hline
\end{tabular}

${ }^{*}$ Expected habitat use from newly translocated population to Lavsan Island.

\section{French Frigate Shoals}

\section{Island Information}

We report elevation data and present topographic maps for French Frigate Shoals (Shark, Tern, Trig, Round, East, Gin, Little Gin, Disappearing Islands and La Perouse Pinnacle). For additional details on SLR models, land cover analysis, vegetation response, projected seabird habitat change analysis, and seabird population dynamics, see Hatfield and others (2012). Aside from Tern (13.8 ha) and East (2.8 ha) Islands, the islands of French Frigate Shoals were less than 2 ha in area (table 1.9). Tern, Trig, East, Gin, and Little Gin Islands support breeding seabirds.

\section{Sea-Level Rise}

The maximum elevations of Shark, Tern, Trig, East, Round, Gin, Little Gin, and Disappearing Islands range from $1.8 \mathrm{~m}$ (Little Gin) to $3.4 \mathrm{~m}$ (Tern), while the mean elevations for these islands range from $0.5 \mathrm{~m}$ (Trig) to $2.3 \mathrm{~m}$ (both Tern and East; table 1.9, figs. 1.37-42). Thirty-five percent of the total land area of French Frigate Shoals was below 2.0 $\mathrm{m}$ and 99 percent was below $3.0 \mathrm{~m}$, excluding La Perouse Pinnacle. Five of the nine islands of French Frigate Shoals were expected to be entirely inundated at $+2.0 \mathrm{~m}$ SLR with the passive inundation model (table 1.9, fig. 1.43). However, total land area loss across French Frigate Shoals was expected to be 12 percent at $+1.0 \mathrm{~m} \mathrm{SLR}$ and 32 percent at $+2.0 \mathrm{~m} \mathrm{SLR}$ (excluding La Perouse). 


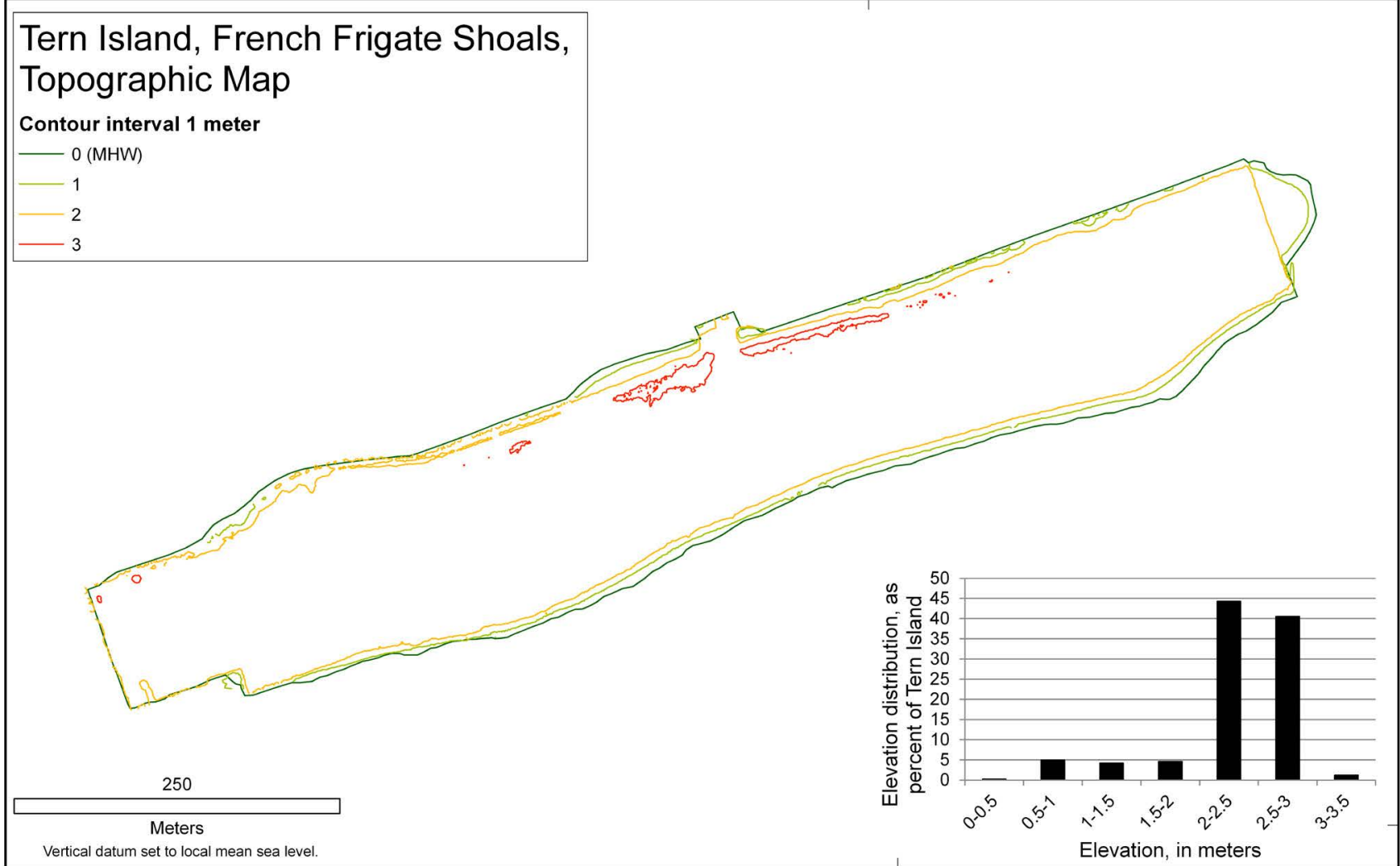

Figure 1.37. Topographic map of Tern Island, French Frigate Shoals, showing mean high water (MHW) and 1-meter $(\mathrm{m})$ contour lines with a graph of the percentage of land in $0.5-\mathrm{m}$ elevation bins. Elevations were determined from U.S. Geological Survey lidar data (2010) with a vertical root-mean-squared error of $0.05 \mathrm{~m}$.

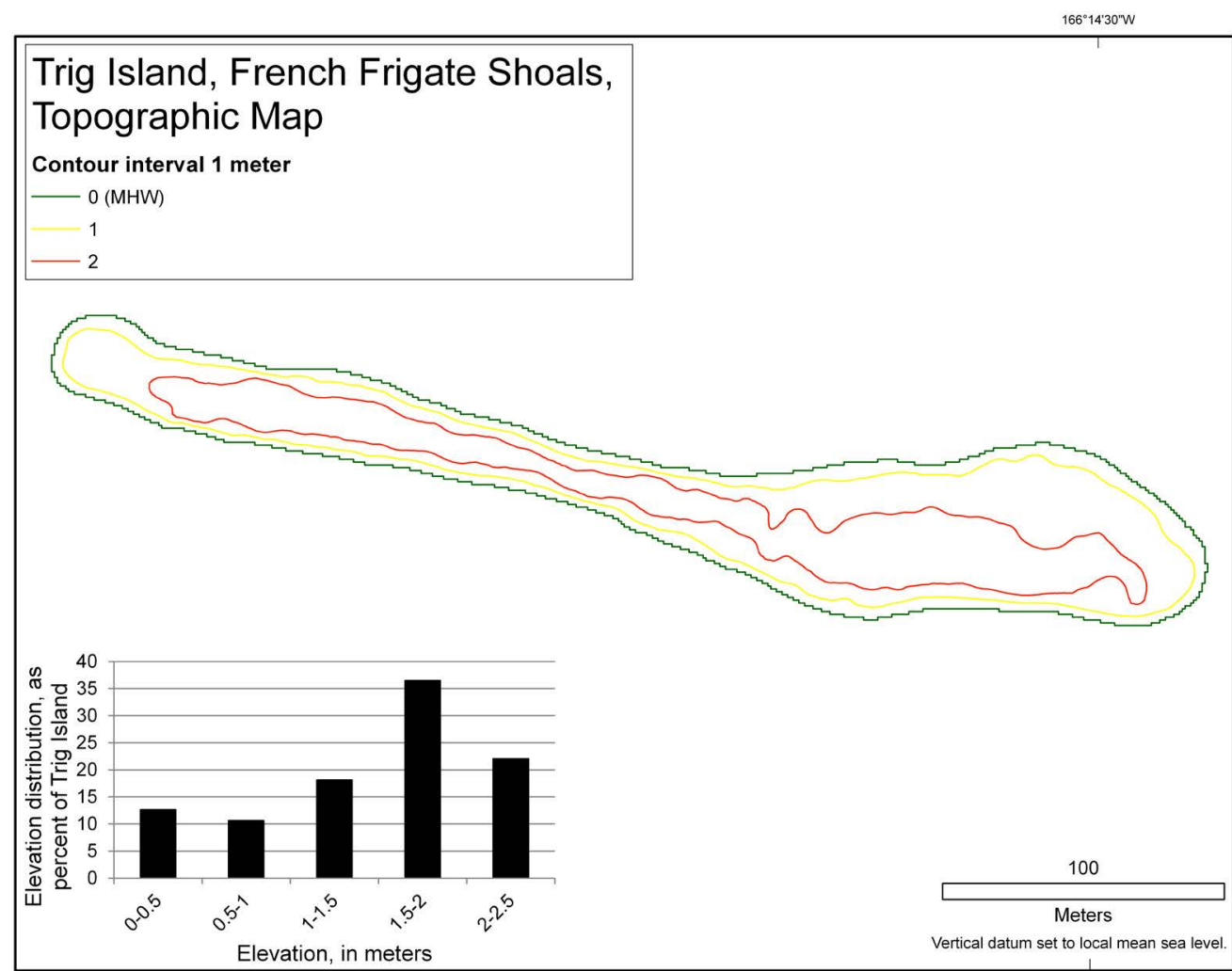

Figure 1.38. Topographic map of Trig Island, French Frigate Shoals, showing mean high water (MHW) and 1-meter (m) contour lines with a graph of the percentage of land in $0.5-\mathrm{m}$ elevation bins. Elevations were determined from PhotoSat (2011) with a vertical rootmean-squared error of $0.15 \mathrm{~m}$. 


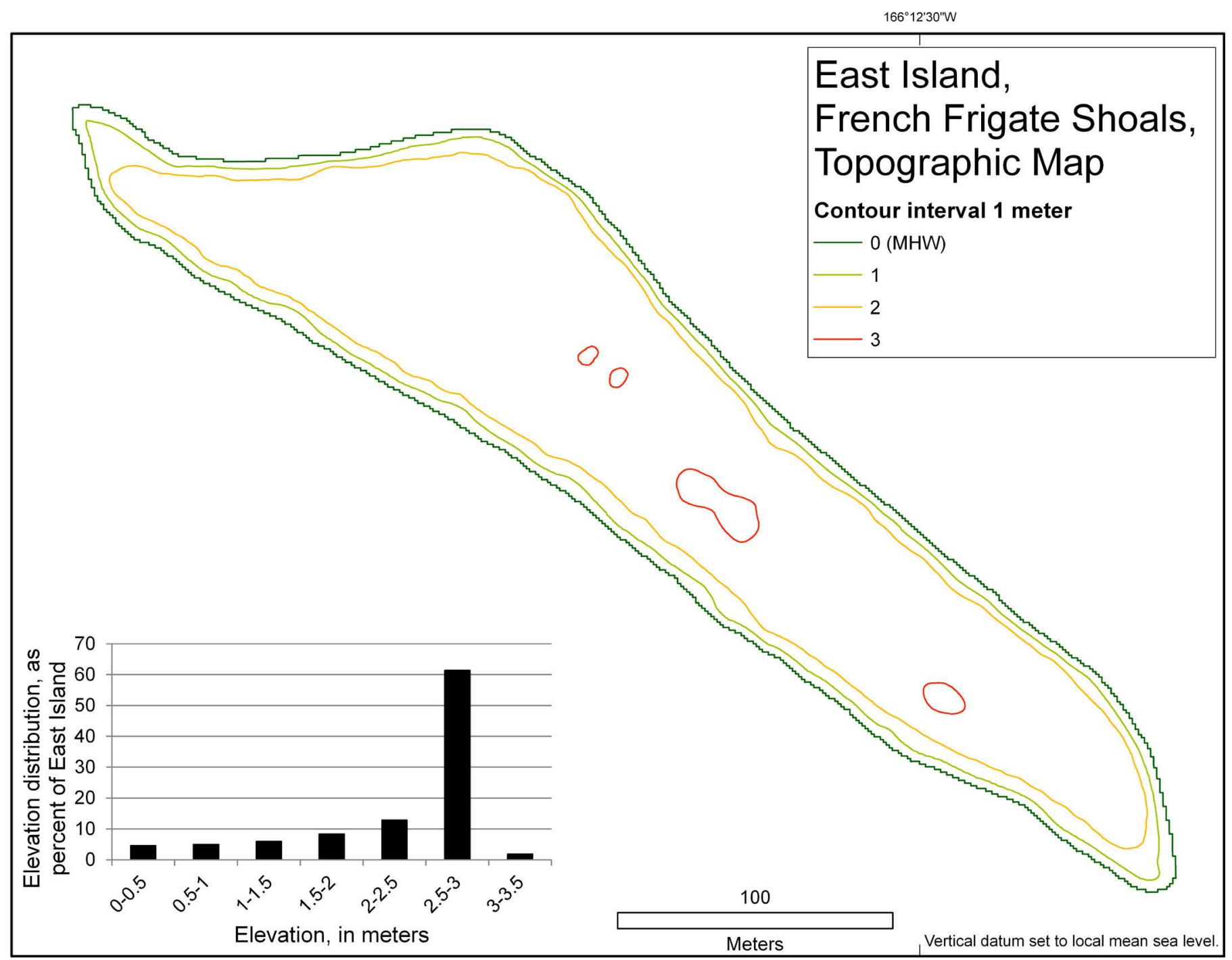

Figure 1.39. Topographic map of East Island, French Frigate Shoals, showing mean high water (MHW) and 1-meter (m) contour lines with a graph of the percentage of land in 0.5-m elevation bins. Elevations were determined from PhotoSat (2011) with a vertical root-mean-squared error of $0.15 \mathrm{~m}$. 
$166^{\circ} 10^{\prime} \mathrm{W}$

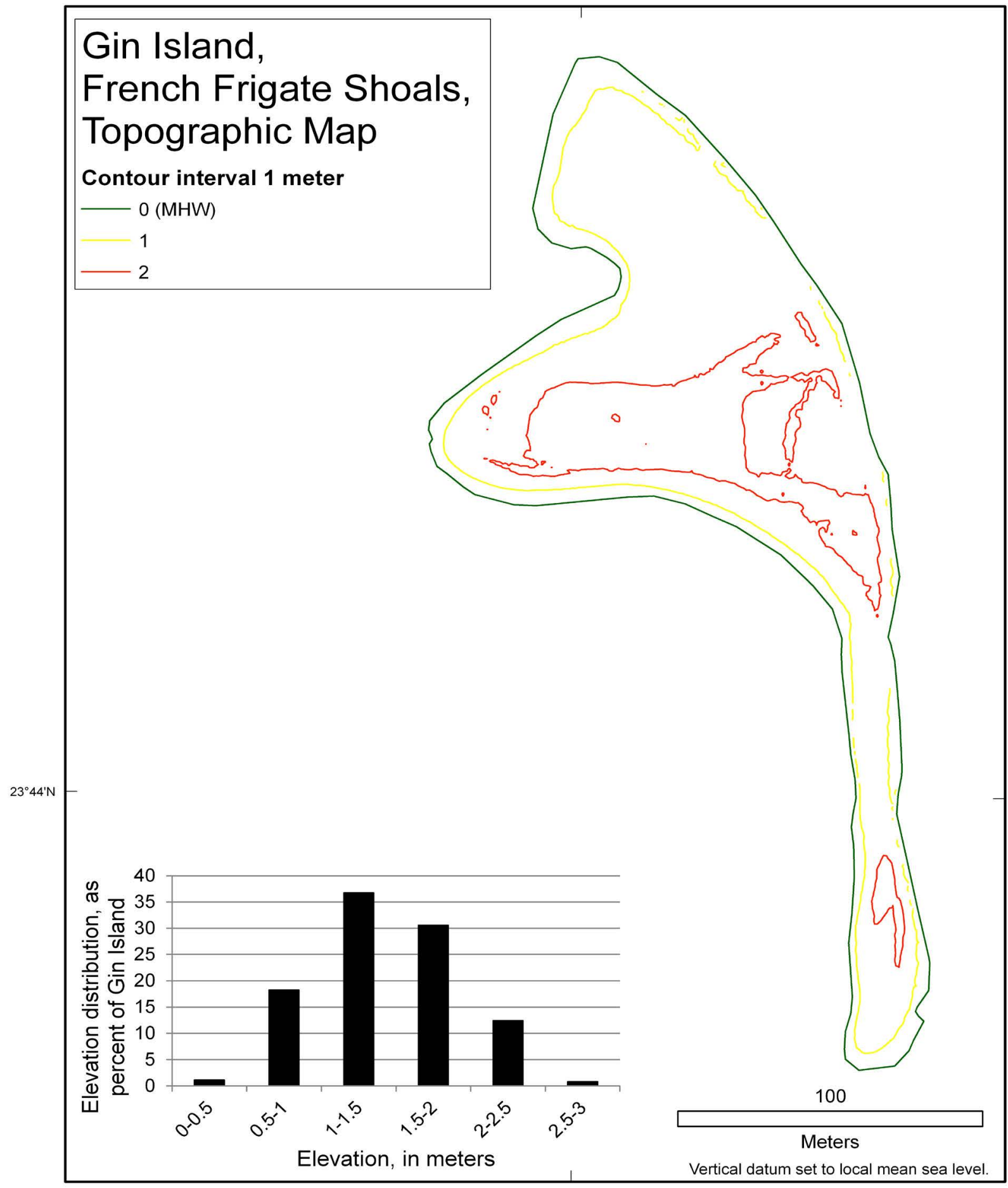

Figure 1.40. Topographic map of Gin Island, French Frigate Shoals, showing mean high water $(\mathrm{MHW})$ and 1-meter $(\mathrm{m})$ contour lines with a graph of the percentage of land in 0.5-m elevation bins. Elevations were determined from U.S. Geological Survey lidar data (2010) with a vertical root-meansquared error of $0.05 \mathrm{~m}$. 


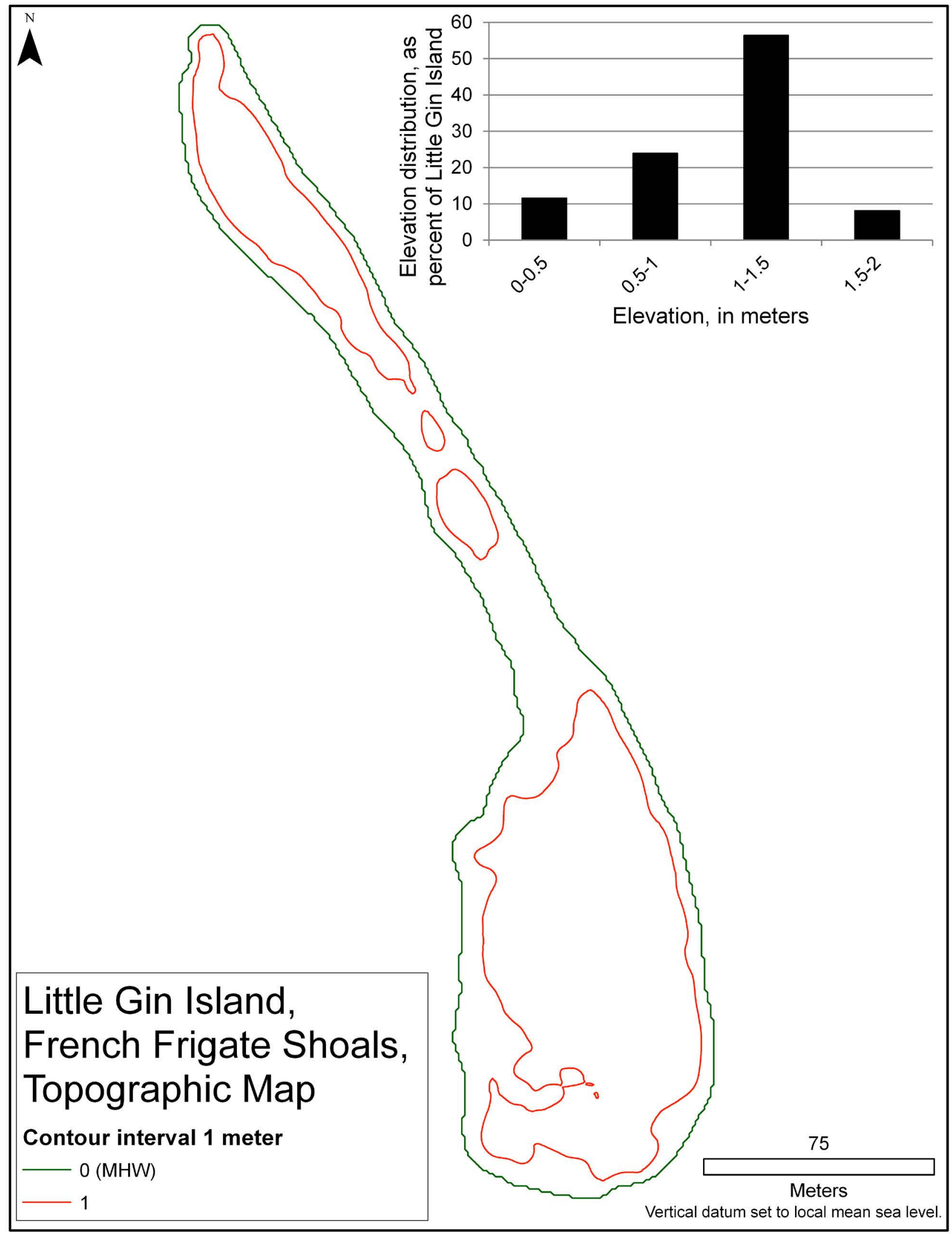

Figure 1.41. Topographic map of Little Gin Island, French Frigate Shoals, showing mean high water (MHW) and 1-meter $(\mathrm{m})$ contour lines with a graph of the percentage of land in $0.5-\mathrm{m}$ elevation bins. Elevations were determined from PhotoSat (2011) with a vertical root-mean-squared error of $0.15 \mathrm{~m}$. 


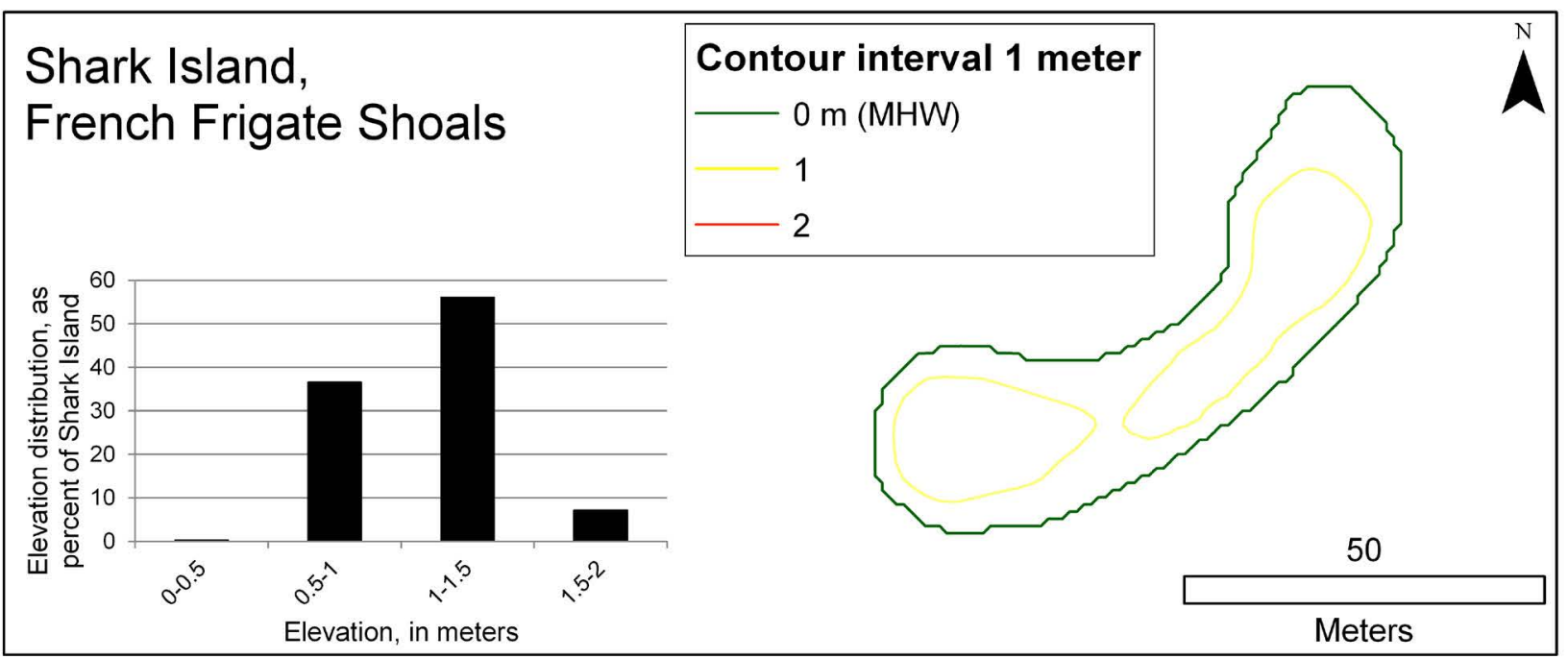

\section{Round Island, \\ French Frigate Shoals}
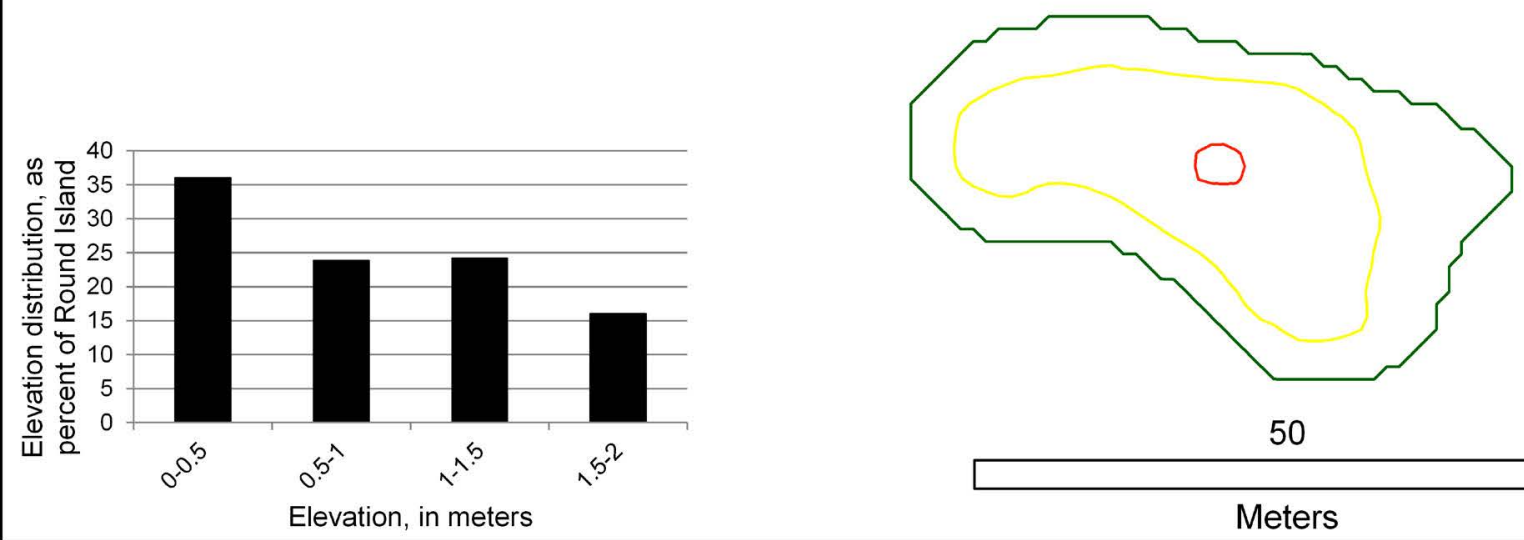

50

Meters

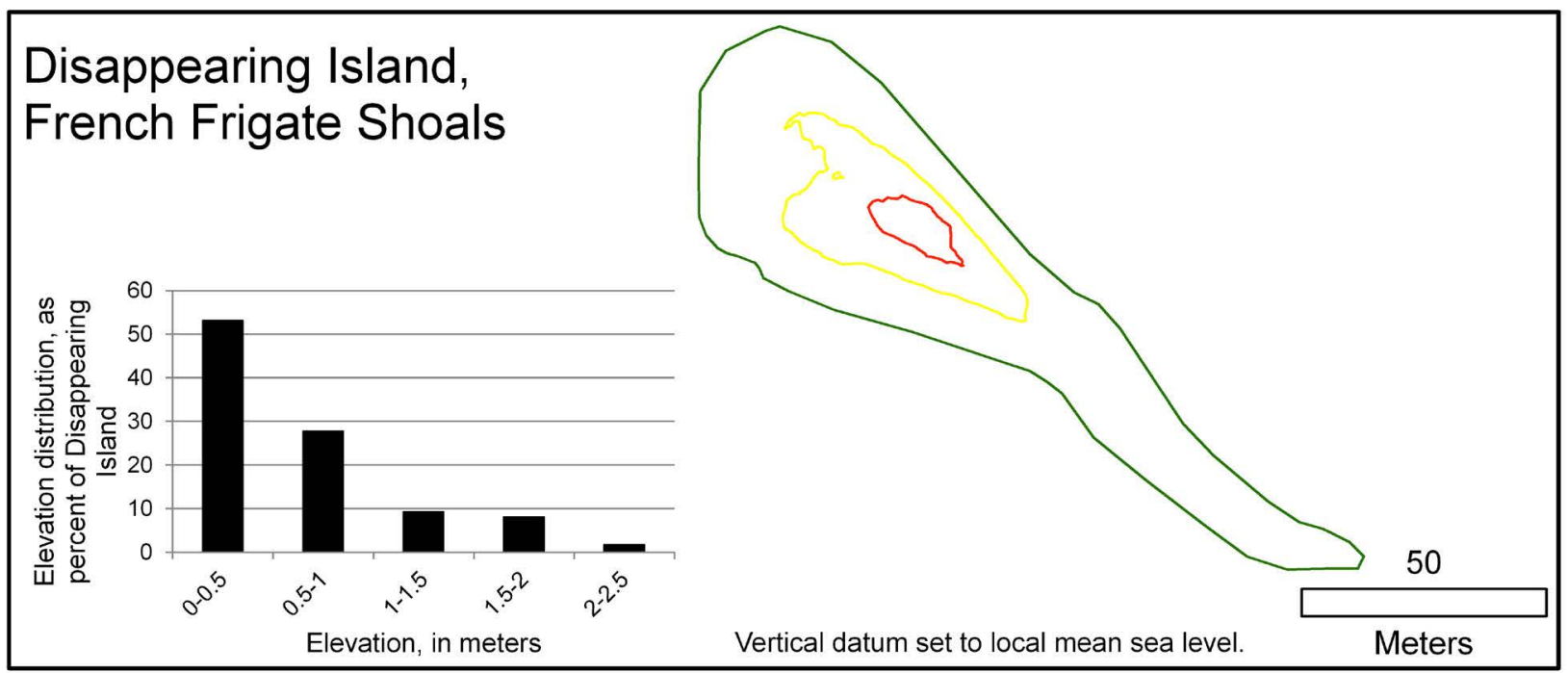

Figure 1.42. Topographic map of Shark, Round and Disappearing Islands of French Frigate Shoals, showing mean high water $(\mathrm{MHW})$ and 1-meter $(\mathrm{m})$ contour lines with a graph of the percentage of land in $0.5-\mathrm{m}$ elevation bins. Elevations were determined from U.S. Geological Survey lidar (Shark and Disappearing [2011]) and PhotoSat (Round [2011]) data with a vertical root-mean-squared error of $0.05-0.15 \mathrm{~m}$. 
$166^{\circ} 19^{\prime} 30^{\circ} \mathrm{W}$

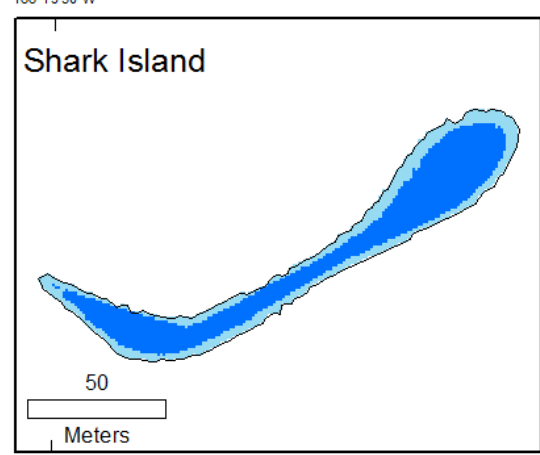

$166^{*} 17 \mathrm{~W}$

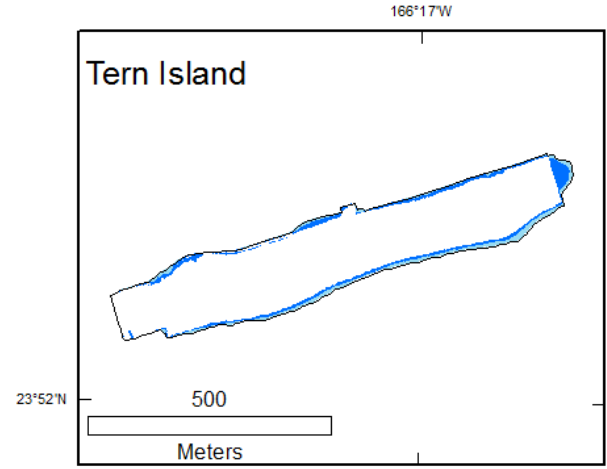

$166^{\circ} 12^{2} 30^{\circ} \mathrm{W}$

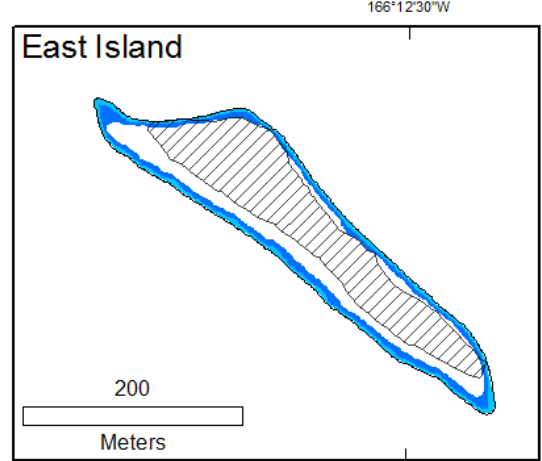

$166^{\circ} 14^{4} 30^{\circ} \mathrm{W}$

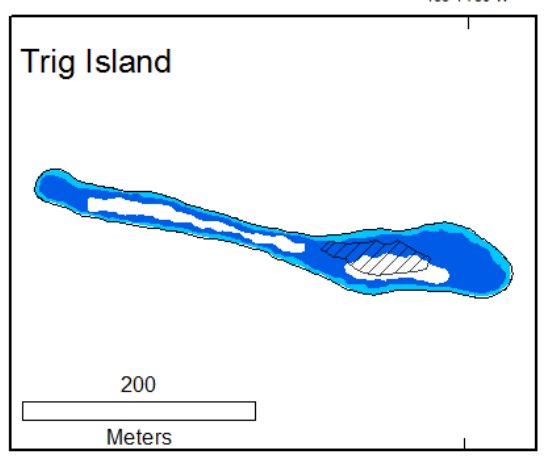

French Frigate Shoals Passive Inundation Scenarios

$\square / A$ Seabird nesting habitat
Human structure
$0.0 \mathrm{~m} \mathrm{(MHW)}$
$+1.0 \mathrm{~m}$ sea-level rise inundation area
$+2.0 \mathrm{~m}$ sea-level rise inundation area
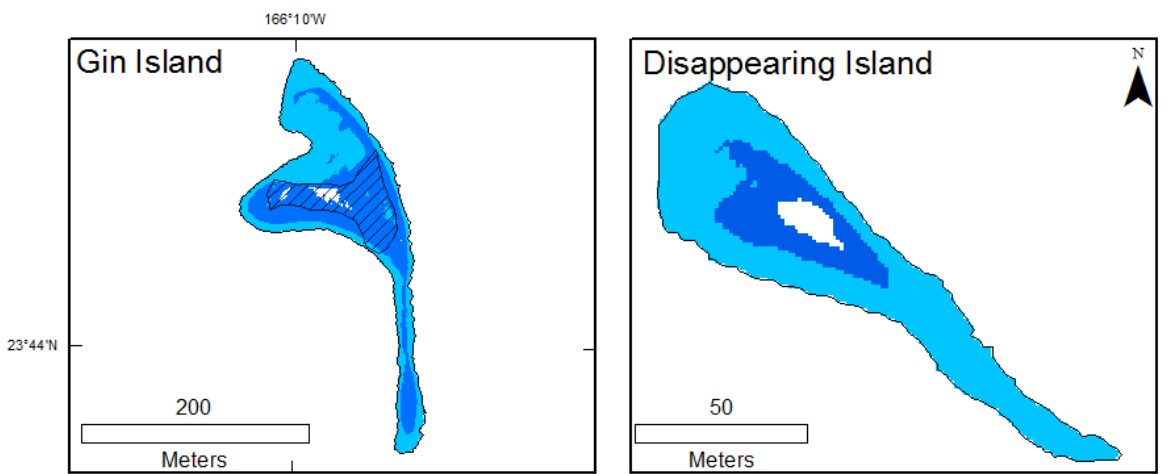

$166^{\circ} 10 \mathrm{w}$
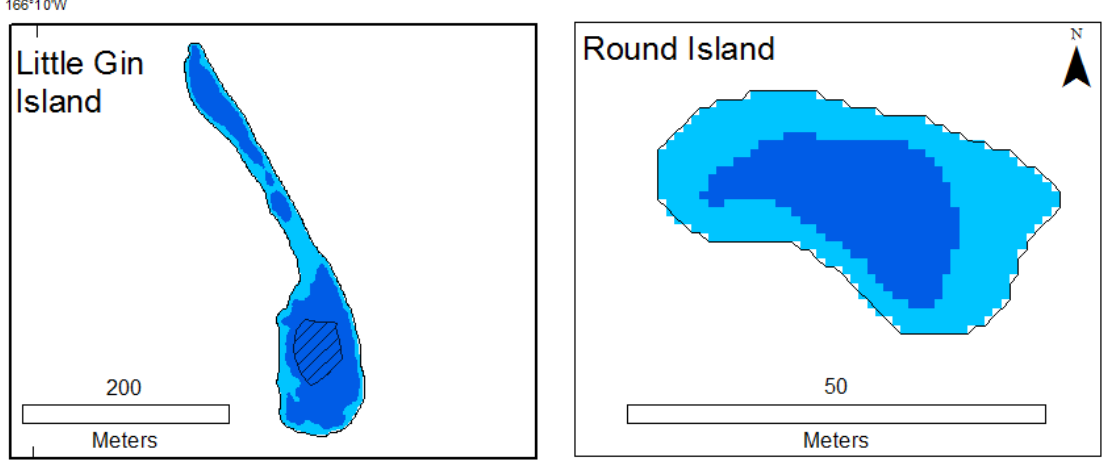

Figure 1.43. French Frigate Shoals passive inundation scenario maps for eight islands at three sea levels: mean high water (MHW), +1.0 and +2.0 meters $(\mathrm{m})$ sea-level rise (SLR). Nesting habitat was mapped from 2011 field data (U.S. Fish and Wildlife Service, unpub. data). Inundation scenarios are based on U.S. Geological Survey lidar- and PhotSat-derived elevations with root-mean-squared errors of $0.05-0.15 \mathrm{~m}$. 


\section{Discussion}

The first quantitative assessment of SLR for the NWHI (Baker and others, 2006) included only three atolls: Pearl and Hermes Atoll (North, Little North, Southeast, Grass, and Seal-Kittery Islands), Lisianski Island, and French Frigate Shoals (only the islands of Trig, East, Gin, and Little Gin). The Baker and others (2006) study examined three different SLR scenarios $(+0.09,+0.48$, and $+0.88 \mathrm{~m})$ based on previous IPCC forecasts and Church and others (2001). Baker and others (2006) analyzed the extent of inundation at each SLR scenario for both MLW and spring tide, using topography derived from ground-surveying techniques and interpolation.

In contrast, our study examined a wider range of sealevel scenarios ranging from 0.0 to $+2.0 \mathrm{~m}$ and twice as many islands. Since our study emphasized terrestrial avian habitat, we conducted our inundation analysis relative to MHW, which is similar to (although somewhat lower than) spring tides. Lastly, our digital elevations were derived from airborne lidar data instead of from ground surveys of the waterline and berm. While the ground surveying techniques used by Baker and others (2006) generally produced data of excellent accuracy at each survey point, spatial coverage was limited and, thus, required extensive interpolation to generate comprehensive topography. In short, given the differences in SLR scenarios and tidal datums, exact comparisons between our findings and the Baker and others (2006) results are impossible, although generalizations can be made.

The calculated total land area for the islands included in both studies differed. The area calculated from our lidar data (201.5 ha) was 6 percent greater than the area used in the Baker and others (2006) study (189.3 ha). At $+0.50 \mathrm{~m}$ SLR and MHW, we predicted a 6-percent loss in total land area for all islands, whereas Baker and others (2006) predicted a 9-percent loss at $+0.48 \mathrm{~m}$ SLR and spring tide. At $+1.00 \mathrm{~m}$ SLR and MHW, we predicted a 13-percent loss in total area, whereas Baker and others (2006) predicted an 18-percent loss at $+0.88 \mathrm{~m}$ SLR and spring tide. Overall, our passive SLR models showed slightly less inundation. However, given that the SLR scenarios, tidal datums, and elevation models differ between studies, these are small differences. For some of the smaller islands, particularly Trig (1.4 ha) and Gin (1.7 ha), it appears that a factor in the difference is variation in initial island sizes between studies, most likely a result of shifting sand, currents, and dynamic island morphology. Consequently, comparable areal losses observed in both studies often result in large differences in an island's proportion of area lost to SLR. Although land cover was not classified in the Baker and others (2006) study, we expect differences in predicted inundation to occur primarily in the beach land cover class due to wave influences and dynamic morphology.

Geomorphic changes such as island migration, accretion and erosion, as identified by Webb and Kench (2010) for other locations in the Pacific, have not been examined for the NWHI and are not included in our predictions. The findings of Webb and Kench (2010) suggest that up to a point reef islands are geomorphically resilient landforms that have remained fairly stable in the last 20-60 years (Webb and Kench, 2010).

Predictions of land area loss and wildlife impacts from SLR varied among the islands of the NWHI. The primary breeding habitats of the endangered Hawaiian monk seal, are beach, bare ground near the coast, and vegetation behind beaches used for shelter (Antonelis and others, 2006; NOAA 2007). Thus, Hawaiian monk seal habitat will likely be sensitive to changes in land cover as a result of beach loss and coastal erosion. The primary nesting area of green turtle is coastal bare ground habitat, often within $8 \mathrm{~m}$ of the high tide line (Niethammer and others, 1997). Because approximately 90 percent of the Hawaiian population of green turtles nest at French Frigate Shoals (Balazs, 1976; Balazs and Chaloupka, 2004; Tiwari and others, 2010), the decreases in nesting area predicted at $+1.0 \mathrm{~m}$ SLR as well as the complete inundation of five islands at $+2.0 \mathrm{~m} \mathrm{SLR}$, are likely to limit nesting habitat for this population if philopatric behavior prevents their dispersal. As sea levels rise and coastal habitat is inundated, vegetation along the coastline, groundwater levels, and turtle nesting density will likely change. These changes along with increasing temperatures are expected to negatively impact turtle nesting (Niethammer and others, 1997; Tiwari and others, 2010).

Across the NWHI, nesting habitat losses predicted with the passive inundation model were similar among avian species, despite differences in habitat use. We expect that additional avian breeding habitat will be lost if land cover shifts dramatically with rising sea levels (LaFever and others, 2007), largely because of the encroachment of beach into areas that are currently breeding habitat with vegetation. Under such conditions nesting habitat losses may not occur uniformly across species. Avian species of the NWHI, such as the Sooty Tern and Brown Noddy, that nest in a broad range of habitat types, do not typically nest coastally, and have broad global distributions will likely be the most resilient to SLR and additional climate change effects in the region.

Reductions in limited habitat types could, however, disproportionally impact habitat specialists that may have limited adaptation potential to respond to habitat changes (Laidre and others, 2008). Specifically, shrub habitat across the NWHI was a limited land cover at most atolls and was entirely absent from most of the smaller islets (for example, all of Pearl and Hermes Atoll and Shark, Trig, Gin, Little Gin, Round, and Disappearing Islands of French Frigate Shoals). As a result, we expect that even small losses in shrub habitat due to SLR and other potential climate change impacts (for example, more frequent storm surge) may negatively affect the dependent shrub-nesting avifauna by further limiting nest-site availability. However, White Terns that readily nest on artificial structures, large rocks, and debris where available (Rauzon and Kenyon, 1984) may be best able to adapt to the loss of shrubs. 
Also specialized in their nesting habitat use are the burrow- and crevice- nesting species of the NWHI (Bonin Petrel, Bulwer's Petrel, Tristram's Storm-petrel, and Wedgetailed Shearwater) that select nesting habitat according to soil types or substrate and depths for their subterranean nests (McClelland and others, 2008; Moore, 2009). Although data limitations prevented us from determining species-specific habitat based on soil characteristics, evidence suggests that potential habitat for the burrowing species is geographically limited at each atoll (McClelland and others, 2008; Moore, 2009). Inundation of substrates favorable for subterranean nests may limit or exclude these species on vulnerable lowlying islands, depending on the spatial relationship between soil characteristics and inundation patterns.

The effects of habitat reduction would be further exacerbated at islands where populations are at or near carrying capacity with density-dependent populations (Gaston and others, 2003). Although very limited avian population data are available throughout the NWHI, studies indicate that the Laysan Teal at Laysan Island and several seabird species at Tern Island are already at maximum carrying capacity (Seavy and others, 2009; Hatfield and others, 2012). Life-history constraints that may influence adaptation to SLR are further discussed in chapter 2 .

Habitat loss on islands that provide nesting habitat for globally important avian populations could have tremendous impacts even for species that utilize a broad range of habitat types. For example, at Southeast Island, Pearl and Hermes Atoll, one of only two islands in the chain where the endemic Laysan Finch persists, 95 percent of finch nesting habitat is expected to be lost here at $+2.0 \mathrm{~m}$ SLR. The Laysan Finch is not capable of unaided inter-atoll dispersal, thus habitat loss at Pearl and Hermes Atoll would likely restrict the range of this endangered species to Laysan Island, an island vulnerable under higher scenarios of SLR (see chap. 2).

At Midway Atoll, substantial habitat loss is predicted for several globally important populations: the endangered Laysan Teal, one of the largest colonies of Bonin Petrel (Moore, 2009), and the world's largest colonies of Black-footed and Laysan albatross, representing one-third and three-quarters of the world's breeding populations, respectively (USFWS data, Flint, 2011). The second largest populations of Black-footed and Laysan albatross, the founder population of Laysan Teal and Laysan Finch, and a newly translocated population of Nihoa Millerbird are all found on Laysan Island. Although high coastal dunes may protect much of Laysan Island from some of the impacts of SLR over the next 100 years, stochastic and extreme events are risks for the globally important populations of these species (see also chap. 2).

Habitat loss from passive SLR will likely be compounded by other forecasted impacts of rapid climate change such as increased temperatures, decreased precipitation, and increased frequency of overwash events and severe storms (Richardson and others, 2009). In addition to erosion caused by wave action, some nesting habitat may be negatively influenced by more frequent wave overwash as many shrub and grass species are intolerant of saltwater; storm events may weaken or slow the rebound of vegetation that, in turn, may further increase erosion.

Increased frequency of storms may also have direct impacts on avian breeding productivity and/or survival (Frederiksen and others, 2008). Storm waves and flooding have been the cause of large-scale failure of albatross nests and adult mortality at Laysan Island and Midway Atoll (Kristof and others, 2011; E. Flint, USFWS, oral commun., 2011). Reproductive failure of Laysan Finches and Laysan Teal at Laysan Island during extensive storms and extreme events have also been documented (Morin, 1992a; Kristof and others, 2011), highlighting the potential negative impacts of increasing storm intensity on NWHI avifauna.

Impacts to NWHI avifauna from rapid climate change may occur with predicted increases in climatic variability that may resemble extreme events similar to El Niño-Southern Oscillation events (IPCC, 2007). The six climate change scenarios examined all predicted increases in temperatures and decreases in precipitation. The NWHI endemic land birds have demonstrated strong demographic responses to climatic variability including reduced rainfall (Morin, 1992a; Reynolds, 2002; Work and others, 2004) and increased temperatures (Reynolds and others, 2007; Work and others, 2010). For seabirds, environmental stochasticity can alter the distribution of prey associated with specific oceanic water masses (Polovina and others, 2001; Bograd and others, 2004) and alter prey composition in foraging areas (Brodeur and others, 1999; Grebmeier and others, 2006). Indeed, many seabird species have reduced survival, productivity, and breeding frequency as a result of altered conditions in their offshore foraging grounds (Schreiber and Schreiber, 1984; Montevecchi and Myers, 1997; Vadenbosch, 2000; Smithers and others, 2004; Congdon and others, 2007; Frederiksen and others, 2008). As such, increased environmental stochasticity is potentially detrimental to the wildlife of the NWHI.

Our results provide the first quantitative assessment of inundation risk and habitat change for many of the lowlying islands of the NWHI using high-resolution topographic data. The potential changes in habitat as a result of SLR may threaten species dependent on the NWHI. Our results show areas most vulnerable to inundation, allowing managers to anticipate where risks are highest. Loss of vegetation may precede permanent inundation, as frequent overwash events often lead to shifts in vegetation patterns, plant dieback, accelerated erosion, and soil instability (Davidson-Arnott, 2005). Past habitat restoration initiatives and shoreline protections have been shown to be beneficial to nesting seabirds throughout the NWHI (Hatfield and others, 2012). Although there are uncertainties regarding climate change impacts and ecosystem response, modeling applications provide tools to better understand the island areas and natural resources most vulnerable to inundation. There is a need for additional climate change adaptation strategies and planning for the four bird species we have identified as potentially in jeopardy from SLR, as well as the marine wildlife dependent on terrestrial breeding habitat of French Frigate Shoals and Pearl and Hermes Atoll, likely to be inundated before 2100 . 


\section{Acknowledgments}

This research and publication was made possible by funding from the U.S. Geological Survey's National Climate Change Wildlife Science Center and Pacific Island Ecosystems Research Center, National Oceanic and Atmospheric Administration's Pacific Services Center and Coastal Services Center, and Papāhanaumokuākea Marine National Monument. We thank center directors D. Beard and G. Tribble for their support. We are grateful to the dedicated staff of the USFWS for long-term data collection and archives of the Hawaiian Islands National Wildlife Refuge. We thank J. Klavitter for facilitating the lidar flights and fuel transportation to Midway Atoll. We thank C. Littnan for helping to facilitate the lidar ground truth data via the Oscar Elton Sette. We thank E. Flint, P. Hartzell, J. Klavitter, R. Born, S. White, C. Rehkemper, and P. Leary from USFWS. We also thank C. Vanderlip, J. Parish, and J. Moore for sharing data and insights on seabird habitat, distribution, and abundance. We thank A. Kristof and M.

Stelmach for data on Laysan Island land cover. We are grateful to $\mathrm{S}$. Nash for extensive editing assistance and review of earlier drafts of this manuscript. We would also like to thank T. Speetjens, and S. Duffy for assistance in formatting and editing and $\mathrm{K}$. Brinck for reviewing an early draft manuscript. We thank L. Fortini and J. Baker for reviewing this manuscript and G. Tribble for advice on atoll hydrology. We also thank L. Slack and J. Hendley (USGS, Menlo Park Publishing Service Center) for editing assistance. 


\title{
Chapter 2
}

\section{Sea-Level Rise and Wave-Driven Inundation Models for Laysan Island}

\author{
By Paul Berkowitz ${ }^{4}$, Curt D. Storlazzi ${ }^{5}$, Karen N. Courtot $^{6}$, Crystal M. Krause ${ }^{6}$, and \\ Michelle H. Reynolds ${ }^{6}$
}

\section{Introduction}

Seabird breeding colonies and resident endemic wildlife on low-lying islands may be threatened by sea-level rise (SLR) and storm waves (Baker and others, 2006; Hatfield and others, 2012; also see chap. 1). In the central and western Pacific, many beaches and islands experience periodic inundation from storm-induced waves; however, until recently, for the Northwestern Hawaiian Islands (NWHI) we lacked basic information to predict the magnitude of physical and biological impacts of SLR and storm waves under either present or future sea-level conditions. New topographic and bathymetric data have allowed us to explore SLR scenarios and the combined impacts of SLR and storm waves for Laysan Island, one of the largest and most ecologically intact islands in the NWHI.

Among the predicted impacts of climate change in the Hawaiian archipelago, including rising sea-surface and atmospheric temperatures, changing oceanic and atmospheric circulation, increased storm frequency, changes in primary productivity, shifting species distributions, and increased disease incidence (Benning and others, 2002; Parmesan, 2006; IPCC, 2007; Friedlander and others, 2009; Loarie and others, 2009), SLR has the potential to be one of the most influential. Satellite observations from 1993 to 2010 (Leuliette, 2012) show global SLR occurring at almost double the rate cited in the Intergovernmental Panel on Climate Change (IPCC, 2007) report. Above-average local SLR rates have been observed near Laysan Island and at the northwest end of the Hawaiian archipelago (fig 2.1; Leuliette, 2012). Recent estimates and syntheses that include both ice melting and thermal expansion suggest a global rise in sea level of about +1.0 meters (m) (above 2000 levels) by the end of the 21 st century (table 2.1); some studies suggest a more extreme rise of $+2.0 \mathrm{~m}$

\footnotetext{
${ }^{4}$ Hawai'i Cooperative Studies Unit, University of Hawai'i at Hilo, Hilo, HI 96720.

${ }^{5}$ U.S. Geological Survey, Pacific Coastal and Marine Science Center, Santa Cruz, CA, 95060.

${ }^{6}$ U.S. Geological Survey, Pacific Island Ecosystems Research Center, Kīlauea Field Station, Hawai'i National Park, HI 96718.
}

in that time frame (Rahmstorf, 2007; Pfeffer and others, 2008; Fletcher, 2009; Vermeer and Rahmstorf, 2009). Beyond the year 2100 , or perhaps sooner according to some scientists, rapid collapses of the Greenland and West Antarctic ice sheets could lead to a SLR of many meters (Overpeck and others, 2006).

Sea-level rise is particularly critical for unconsolidated coral atoll islets, many of which have maximum elevations of less than $3 \mathrm{~m}$ above present sea level, but SLR is also significant for larger carbonate islands such as Laysan. Rising sea levels will likely exacerbate the impacts of storms and wave action on coastlines, primarily by reducing wave-energy dissipation (wave breaking) at the reef crest and increasing water depth relative to hydrodynamic roughness (that is, how the irregular nature of the seabed imparts drag on water flow) over the reef flat (see fig. 2.2). By reducing wave-energy dissipation at the reef crest and over the reef flat, SLR will cause longerwavelength and larger waves to directly impact the coastline and potentially drive coastal erosion. These larger waves at the shoreline increase the potential for wave-driven inundation that can extend inland considerable distances. The maximum vertical extent of waves on a beach, referred to as wave "runup," is primarily a function of wave height, wavelength, and beach slope. Because storm wave heights and wavelengths vary in time and space, and corals reefs are spatially heterogeneous, wave-driven and SLR-induced inundation will also vary spatially and temporally. This variation is particularly large for Pacific Ocean islands and atolls that are exposed to waves in excess of $5 \mathrm{~m}$ numerous times each year (U.S. Army Corps of Engineers (USACE), 2011).

Studies to date, including those presented in chapter 1, Hatfield and others (2012), and work by Baker and others (2006), describe SLR threats for several NWHI using passive inundation models to simulate flooding of the islands (fig. 2.3). These passive models, often referred to as "bathtub" models, do not simulate the cumulative effects of SLR and stormdriven waves, or the associated impacts on the landscape or biodiversity. The National Oceanic and Atmospheric Administration's (NOAA) Coastal Inundation Mapping Guidebook $(2006 ; 2009)$ draws a distinction between two types of 


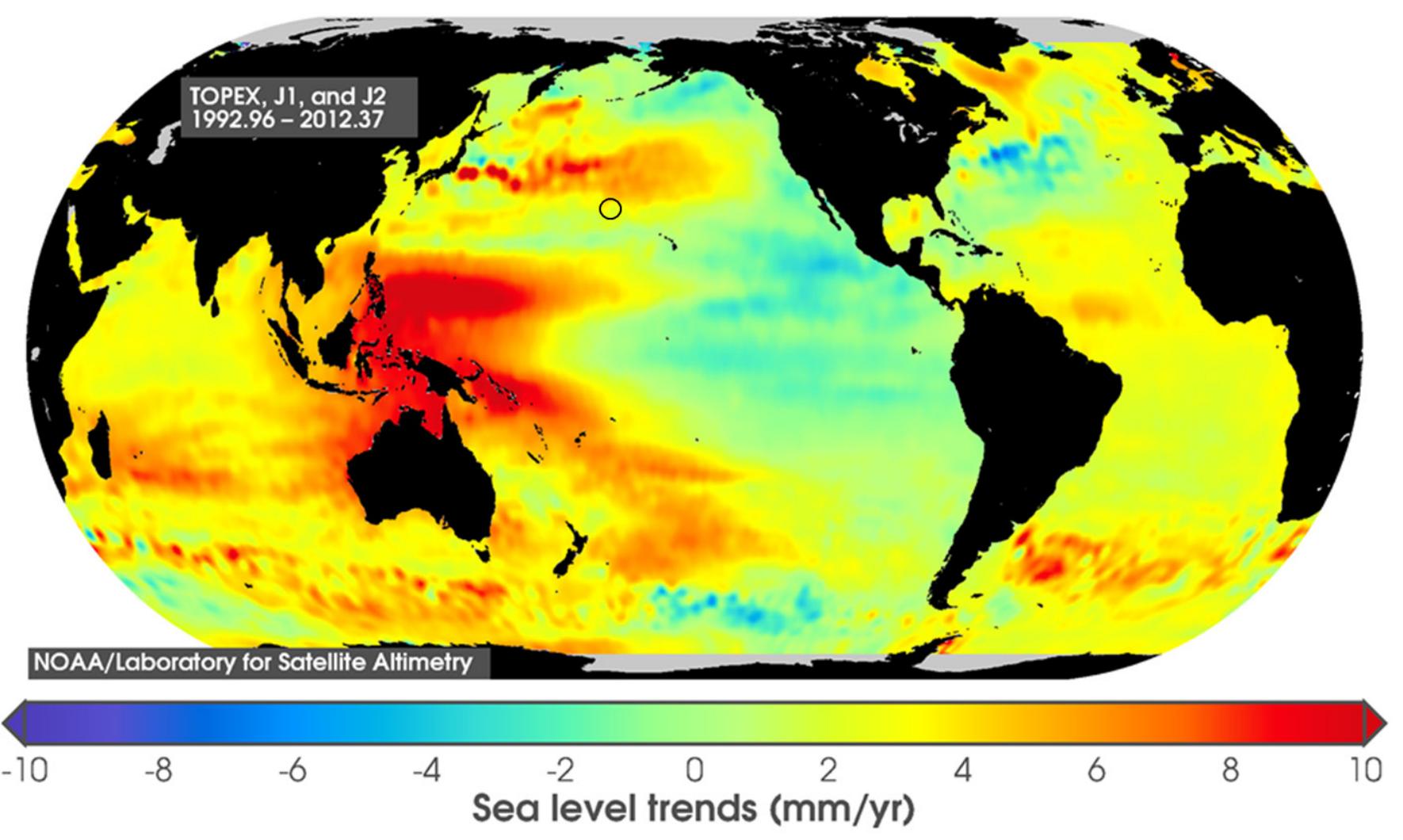

Figure 2.1. Observed trends in sea level between 1993 and 2010 (Leuliette, 2012), in millimeters per year (mm/yr). Note the high rates (greater than $5 \mathrm{~mm} / \mathrm{year}$, displayed in orange and red) observed in the central and western North Pacific Ocean, near the northwest end of Papahānaumokuākea Marine National Monument. On this global map, we depicted Laysan Island's approximate location with a small circle that falls just south of an area of rapid sea-level change.

Table 2.1. Recent published estimates of sea-level rise (in meters) by the end of the 21st century. [The numerical precision of each estimate reflects the original publication]

\begin{tabular}{lll}
\hline \multicolumn{1}{c}{ Study } & \multicolumn{1}{c}{$\begin{array}{c}\text { Estimated range of SLR by 2100 } \\
\text { (meters) }\end{array}$} & \multicolumn{1}{c}{ Author's notes } \\
\hline $\begin{array}{l}\text { Vermeer and Rahmstorf, } \\
2009\end{array}$ & +0.75 to +1.90 (above 1990 level) & Central estimate $=+1.24 \mathrm{~m}$ \\
Pfeffer and others, 2008 & +0.8 to +2.0 (above 2008 level) & $\begin{array}{l}\text { More likely toward the lower end of } \\
\text { range }\end{array}$ \\
Rahmstorf, 2007 & +0.50 to +1.40 (above 1990 level) & \\
\hline
\end{tabular}




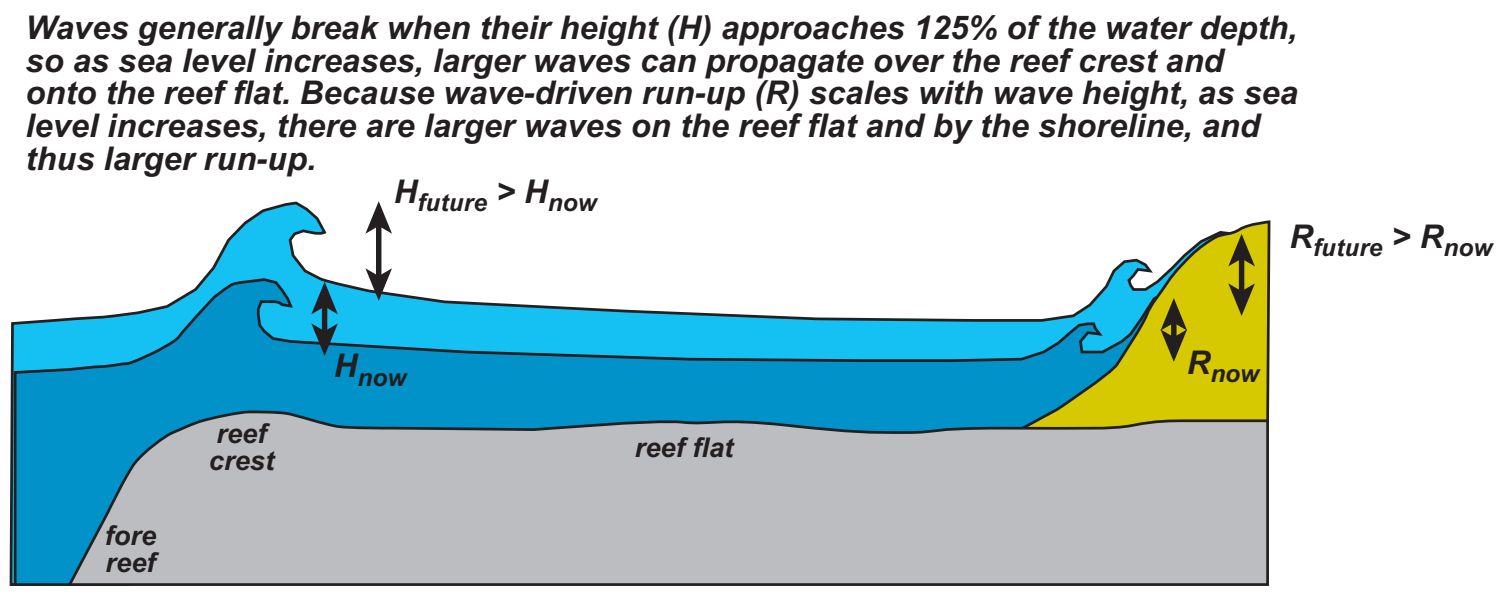

$\square=$ Atoll islet

= Atoll carbonate platform

$\square=$ Present sea level

= Future elevated sea level

Figure 2.2. Schematic diagram showing the variation in waves and resulting wavedriven run-up at two sea levels. At higher sea levels, wave breaking at the reef crest decreases while water depth relative to hydrodynamic roughness over the reef flat increases. Consequently less wave energy is attenuated over the reef crest and reef flat, resulting in more wave energy (larger wave heights and longer wavelengths) reaching the shoreline, which results in higher wave-driven water levels at the shoreline.

inundation modeling depending on the type of water-surface model employed (single-value and modeled water surfaces). The first type of model represents a passive approach, whereby all land below a certain elevation (for example, a level identified in the 2007 IPCC report) and hydrologically connected to the ocean is flooded. The second type of model, applied to Laysan, relies on a more complicated water-surface model to refine estimates of inundation depth and inundation extent (NOAA, 2009). The latter model is referred to as a dynamic or wave-driven inundation model.

Inundation scenarios derived from either passive or dynamic surfaces simplify the wide range of possible impacts of rising sea level. While passive inundation represents an important element of SLR, coastal areas and islands are likely to be affected by a broader, more complex and interrelated set of processes including the following: loss of land due to erosion; island breaching and segmentation; wetland flooding or accretion; saltwater intrusion; and increased frequency of storm flooding (Gesch and others, 2009). The unique characteristics of a particular location affect the relative importance of each of these processes. If data were available, a more comprehensive modeling approach could include sediment transport, morphological changes to the island, density-driven flows, and currents. Data requirements for such a model would include substrate analyses, grain size, water current measurements, and detailed hydrodynamic roughness (Deltares (Dutch Institute for Delta Technology), 2011). In this study, we focused primarily on passive SLR and wave-driven inundation, employing a hydrodynamic numerical model to estimate the combined impacts of SLR and wave-driven inundation on the land cover, vegetation, and selected breeding birds of Laysan Island.

The geomorphological history and isolation of the NWHI have led to the development of extraordinary marine and terrestrial ecosystems, with a high degree of ende-

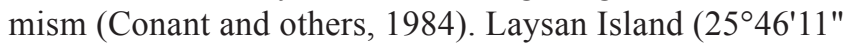
$\mathrm{N}, 171^{\circ} 44^{\prime} 00^{\prime \prime} \mathrm{W}$, ) is the second largest landmass in the NWHI (based on recent ligar data, see chap 1). The biota of Laysan Island remained largely free of anthropogenic disturbance until the late 1800s. By 1900, human impacts to the island included guano mining and rabbit ranching, activities that triggered numerous species extinctions (Ely and Clapp, 1973). Protection of this ecosystem as a bird reserve occurred in 1909 (Executive Order 1019). Additional conservation measures have been implemented to protect the ecological integrity of this island, including the creation of the Hawaiian Islands and Midway Atoll national wildlife refuges (Executive Order 13022; National Wildlife Refuge System Administration Act, 1966, as amended) and Papahānaumokuākea Marine National Monument (PMNM; Presidential Proclamation 8031). Today (2012), Laysan supports 17 species of breeding seabirds and numerous endangered, threatened, and candidate vertebrate species of concern to the U.S. Fish and Wildlife Service (USFWS) or International Union for Conservation of Nature (IUCN): 
Hawaiian monk seals (Monachus schauinslandi), green turtles (Chelonia mydas), Black-footed Albatross (Phoebastria nigripes), Laysan Albatross (P. immutabilis), Christmas Shearwater (Puffinus nativitatis), Tristram's Storm-petrel (Oceanodroma tristrami), Laysan Teal (Anas laysanensis), Laysan Finch (Telespiza cantans), and the recently introduced Nihoa Millerbird (Acrocephalus familiaris kingi; Friedlander and others, 2009; Kristof and others, 2011). Additionally, Laysan Island is designated as critical habitat for two endangered plants: Cyperus pennatiformis var. bryanii and Pritchardia remota (see chap. 1).

Although Laysan Island is one of the older islands in the archipelago, estimated at 20.7 million years old (Clague, 1996), it remains one of the higher islands in the NWHI with a maximum elevation of $10.7 \mathrm{~m}$ above mean sea level (see chap. 1). Laysan contrasts sharply with the lower-lying open and closed atolls in the region. Laysan has substantially more emergent land (412.0 hectares (ha), including the lake basin) than most islands within the monument, has the largest natural lake in the Hawaiian Islands, and has less lagoon habitat (approximately 57 square kilometers $\left(\mathrm{km}^{2}\right)$ in waters less than $100 \mathrm{~m}$ deep) than any nearby island or atoll, including Kure Atoll, Midway Atoll, Pearl and Hermes Atoll, Lisianski Island, and French Frigate Shoals (Friedlander and others, 2009).

Laysan's unique topographic and bathymetric characteristics will influence how climate change affects the future landscape of the island. Our study is the first to simulate wave-driven inundation with a three-dimensional hydrodynamic model to predict the combined impacts of SLR and wave-driven water levels on the physical features and selected biological resources of Laysan Island. We focus on the breeding birds of Laysan Island, yet this assessment of inundation from SLR and storm-generated waves also can be applied to identify vulnerable habitat for other species as well as to identify other vulnerable sites such as field camps and infrastructure.

\section{Methods}

\section{Storm Wave Models}

Ocean surface gravity waves ("storm-induced waves") were simulated with a coupled wave and water-level hydrodynamic model, the Delft3D WAVE module (Deltares, 2011). This WAVE module has been shown to realistically model the propagation and breaking of waves over Pacific coral reefs (Lowe and others, 2005; Lowe and others, 2009; Hoeke and others, 2011) and is described in detail by Booij and others (1999), Holthuijsen and others (1993), and Ris and others (1999).

Physical processes such as bottom friction, depth-induced wave breaking, and wave-wave interactions are included in the simulations. The results of the wave simulation include wave height, peak spectral period (the time period between successive wave crests at which wave energy reaches its maximum), and mass fluxes (rate of mass flow per unit area).

\section{Primary Wave Sources}

We developed a wave climatology based on primary wave sources (North Pacific, Trade Wind, South Pacific, and Kona storms) as well as hurricanes. No buoys with long-term directional wind and wave data exist for the PMNM; thus we used data from the U.S. Army Corps of Engineers (USACE, 2011) Wave Information System for a location $\left(28^{\circ} 00^{\prime} \mathrm{N}\right.$, $\left.174^{\circ} 00^{\prime} \mathrm{W}\right)$ approximately 375 kilometers $(\mathrm{km})$ northwest of Laysan Island (figs. 2.4-7). These data were generated by running wave-forecast models on past events, hence the term "hindcast" data. Based on methodology described in detail by Storlazzi and Wingfield (2005) and Storlazzi and Reid (2010), we analyzed these data (hourly wave height, wave period, wave direction, wind speed, and wind direction) from 1981 to 2004 for the top 5 percent storm conditions for winter and summer. The different sets of conditions helped to constrain the combinations of wave heights, wave periods, and wave directions, above which possible damage to terrestrial habitats might occur. In our final analysis for Laysan Island, we analyzed winter conditions since these seasonal conditions generally encompassed the worst-case scenario according to the USACE wave data.

\section{Elevation and Bathymetry}

As boundary input to the wave and inundation models, we created two seamless topographic/bathymetric grids of differing scales. The first grid covered a broad area around Laysan Island to water depths of approximately 3,000 m and had a spatial resolution or grid cell size of $100 \times 100 \mathrm{~m}$ (fig. 2.8). The second grid had a finer spatial resolution $(20 \times 20 \mathrm{~m}$ cell size) encompassing all land area plus the surrounding ocean to a depth of approximately $30 \mathrm{~m}$ (fig. 2.9). Extensive pre-processing was required to generate these topographic/ bathymetric grids from a wide range of data sources and formats including the following: bathymetric 20 -m gridded data from the Pacific Island Benthic Habitat Mapping Center (PIBHMC, 2011), that is, synthesized pseudo-bathymetric grids derived from IKONOS satellite imagery (in depths of 0-16 m) and multibeam sonar (in depths of 20-5,000 m); two raster navigational charts (19019 and 19442, sections 2-3) and one electronic navigational chart (US1HA01M) from NOAA's Office of Coast Survey (2011); a bare-earth 1-m digital elevation model (DEM) derived from photogrammetric methods by PhotoSat Information Ltd. (2010); and a digitized coastline based on a May 18, 2010 WorldView-2 satellite photo from DigitalGlobe Inc. (2010). Lidar (light detection and ranging) elevation data were not available at the time of analysis; consequently we used elevation data derived from satellite stereo pairs. When two or more data sources covered the same area, we excluded the less reliable sources from analysis. In general, older navigational chart data were used only to fill in data gaps between the newer IKONOS-derived and multibeam sonar data. After converting all depths and elevations to 
Total Water Level = Mean Sea Level + Sea-level Rise + Set-Up + Run-Up = Limit of Inundation

Set-Up = a rise in sea level above the mean sea level inshore of the initial point of wave-breaking produced by cross-shore gradients in momentum flux Run-Up = maximum vertical extent that wave-driven swash reaches above the water level [mean sea level + set-up]

\section{1) GIS-based Passive Inundation Model}
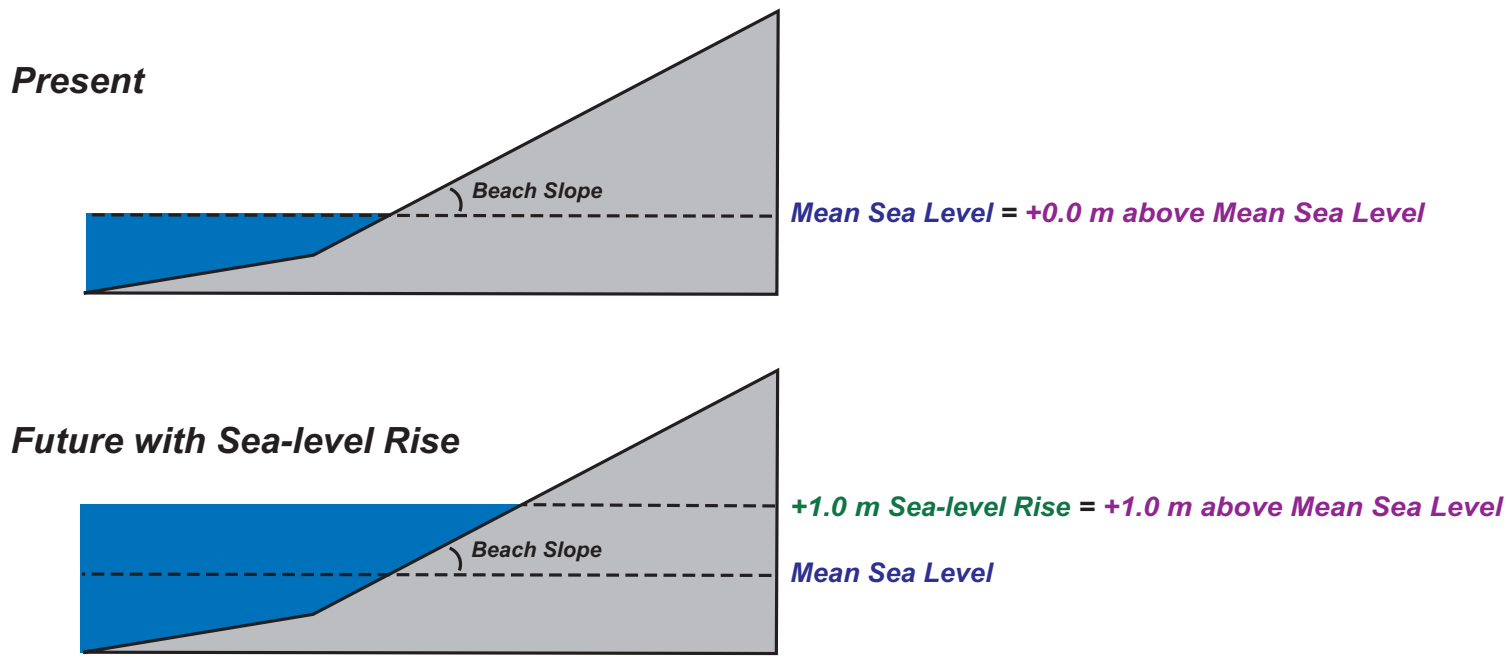

\section{2) Dynamic Wave-driven Inundation Model}
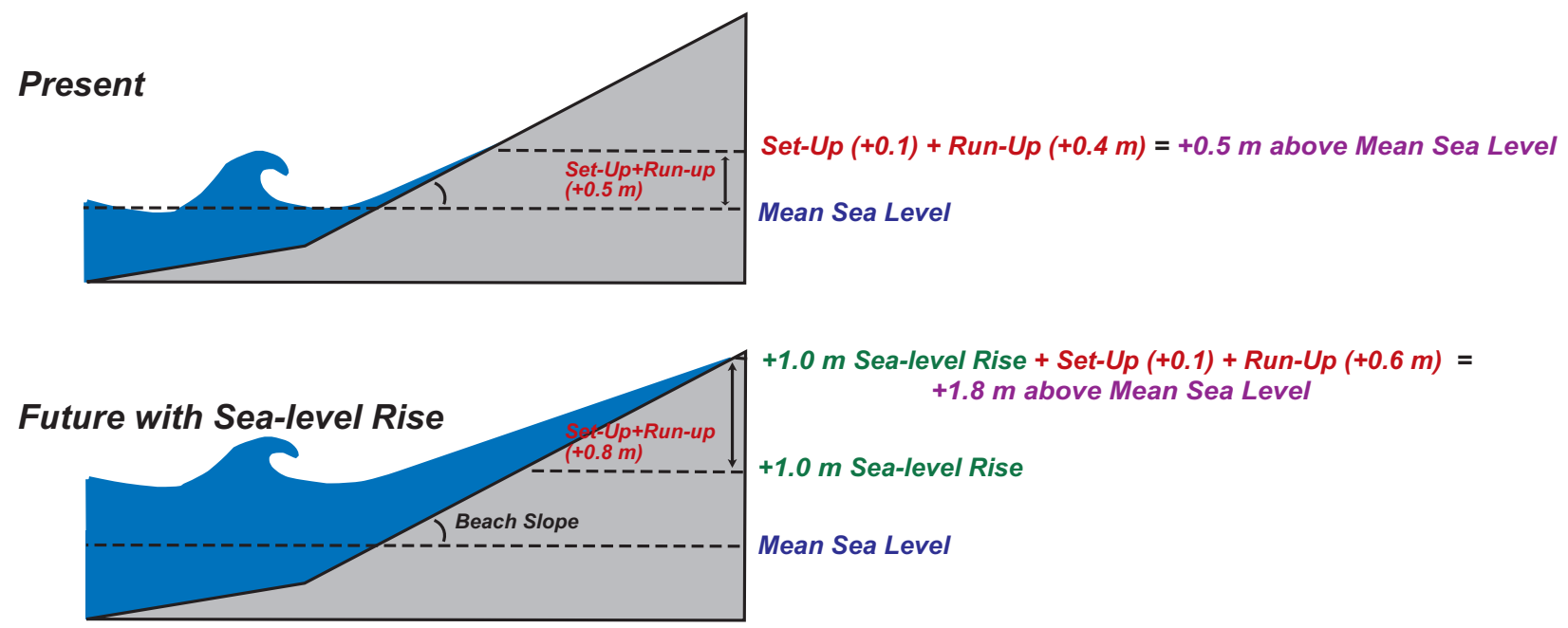

Figure 2.3. Schematic diagram comparing (1) Geographic Information System (GIS)-based passive inundation models (also known as "bathtub" models) with (2) dynamic wave-driven inundation models that incorporate wave-driven water levels (employed in this study). Most sea-level rise models employ passive inundation modeling techniques, which do not account for wave-driven water levels. Abbreviations: $\mathrm{m}$, meters. 
BLUE $=$ all data, RED $=$ top $5 \%$ of data
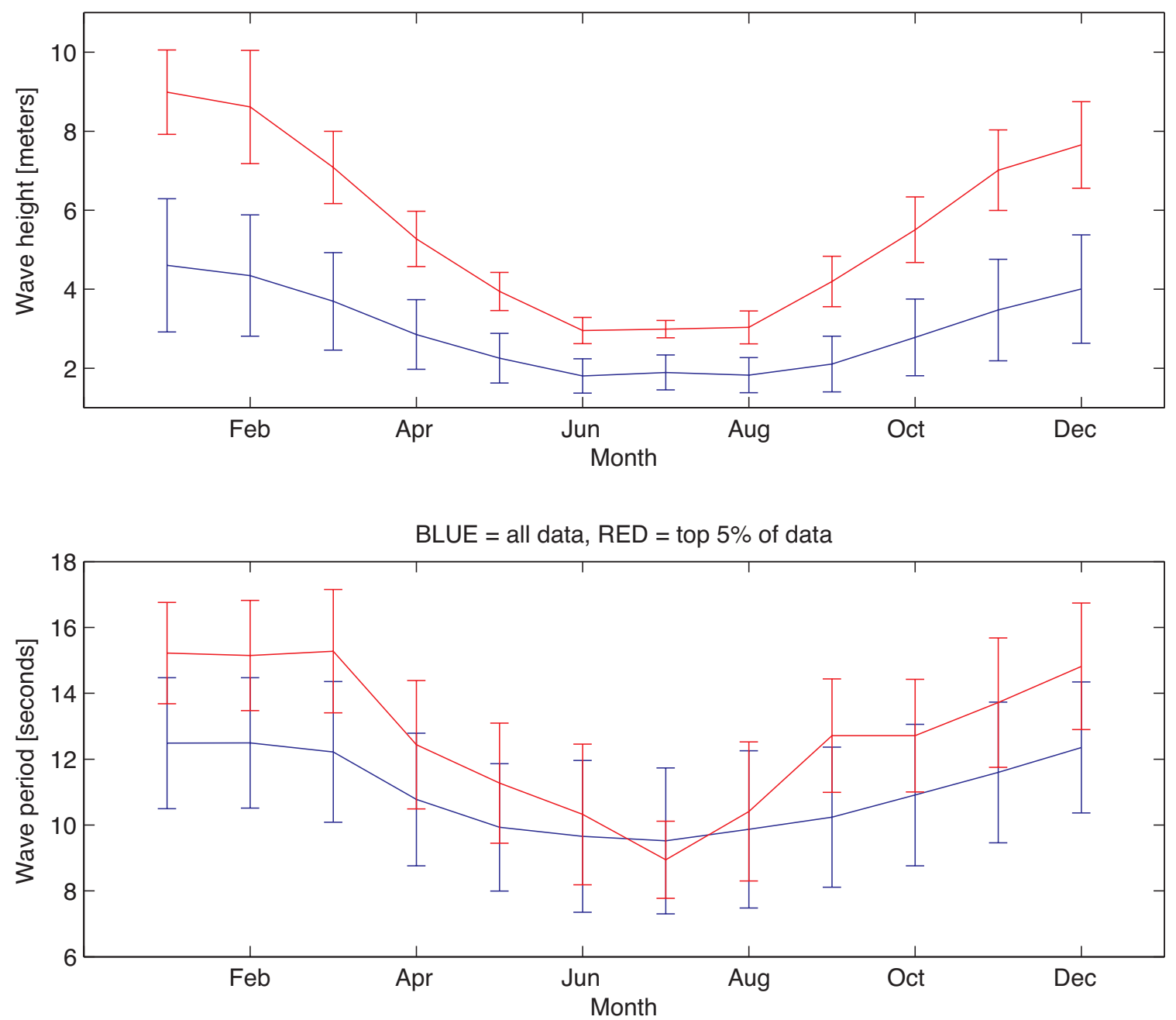

BLUE = all data, RED = top $5 \%$ of data

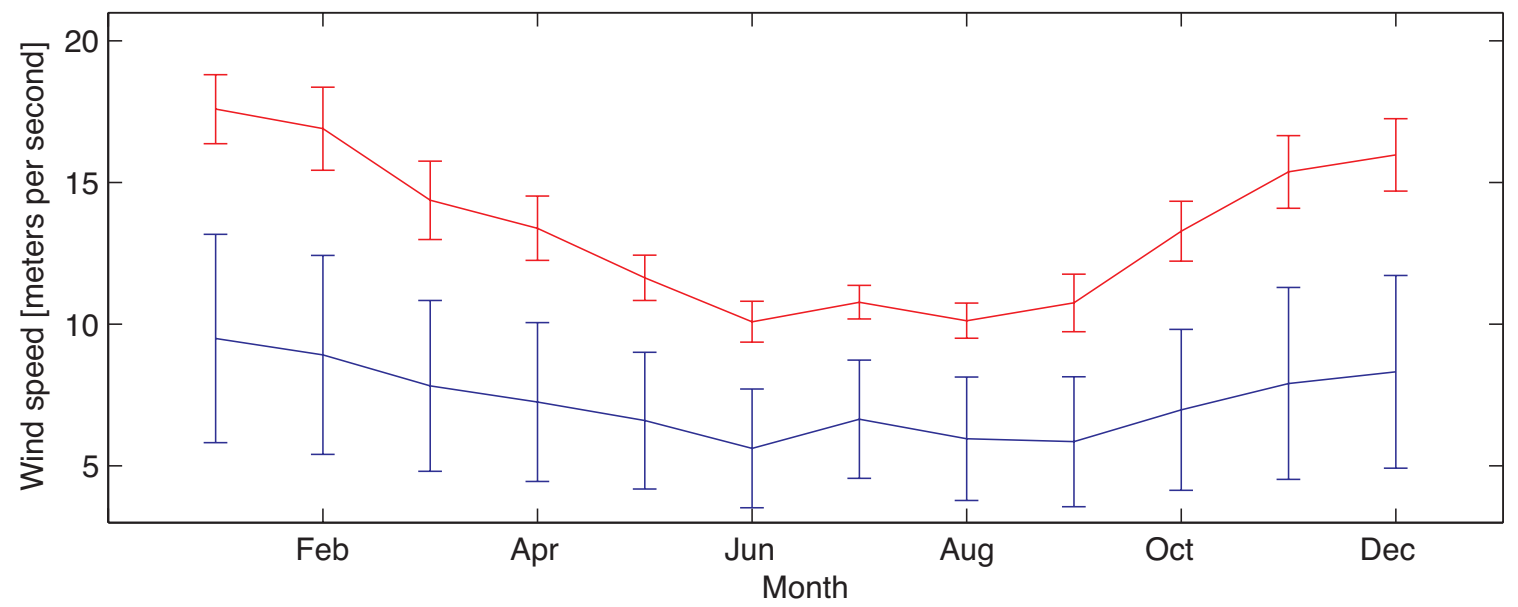

Figure 2.4. Time series showing monthly variations in Pacific Ocean wave heights, wave periods, and wind speeds (means and 1-standard deviation error bars) by month, based on U.S. Army Corps of Engineers (2011) data for the years 1981 to 2004 at a location $\left(28^{\circ} 00^{\prime} \mathrm{N}\right.$, $174^{\circ} 00^{\prime} \mathrm{W}$ ) approximately 375 kilometers northwest of Laysan Island. These hindcast data were used to simulate wave-driven water levels during periods of high-wave energy for various sealevel rise scenarios. 


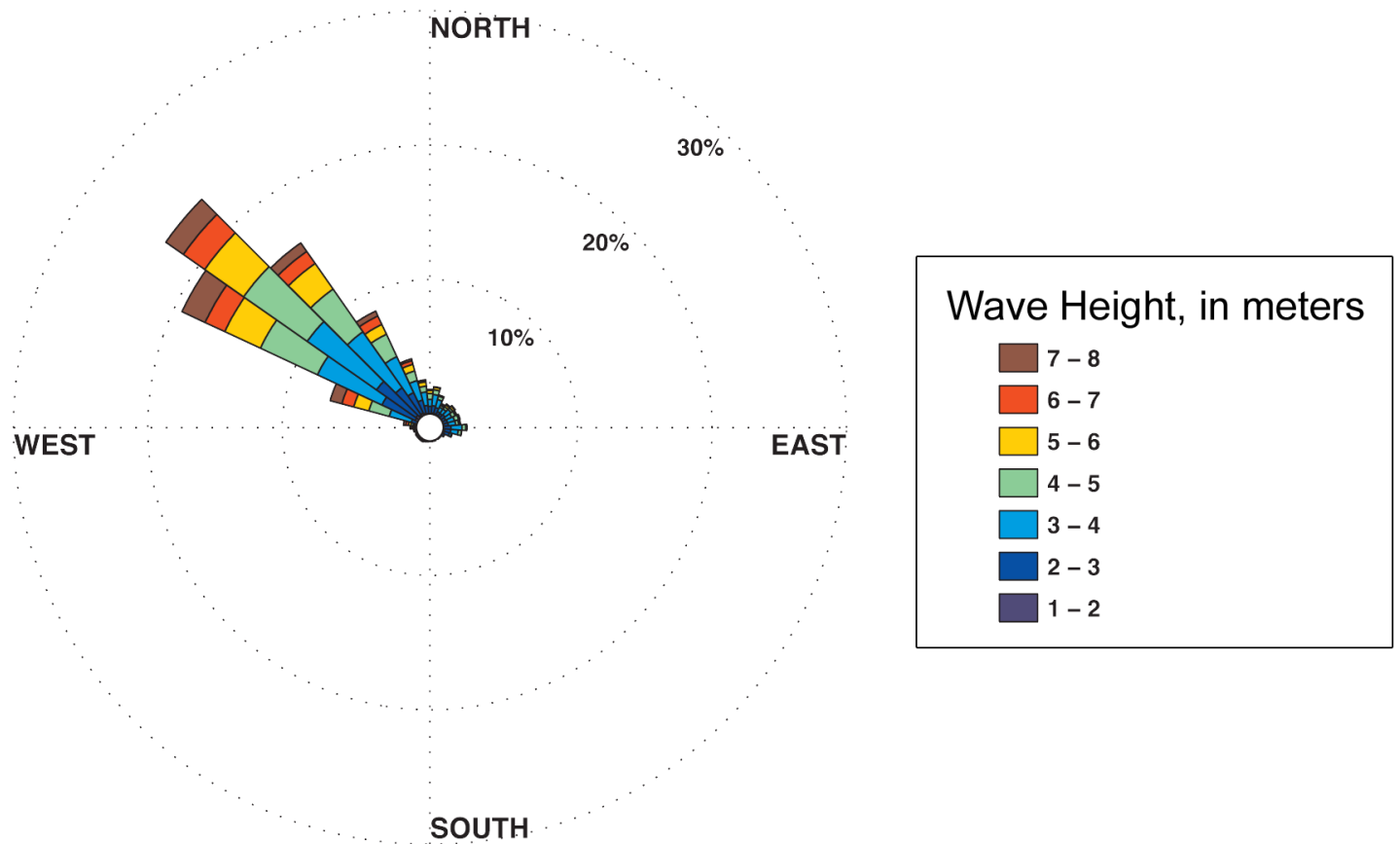

Figure 2.5. Compass plot of variation in wave height in meters $(\mathrm{m})$, by wave direction (or compass orientation), based on U.S. Army Corps of Engineers (2011) data for the years 1981 to 2004 at a location $\left(28^{\circ} 00^{\prime} \mathrm{N}\right.$, $174^{\circ} 00^{\prime} \mathrm{W}$ ) approximately 375 kilometers northwest of Laysan Island. Frequency of occurrence (in percent) increases outward from the center of the compass plot. Wave height data, along with wave period (fig. 2.6) and wind speed (fig. 2.7) data, were used to simulate wave-driven water levels under various sea-level rise scenarios.

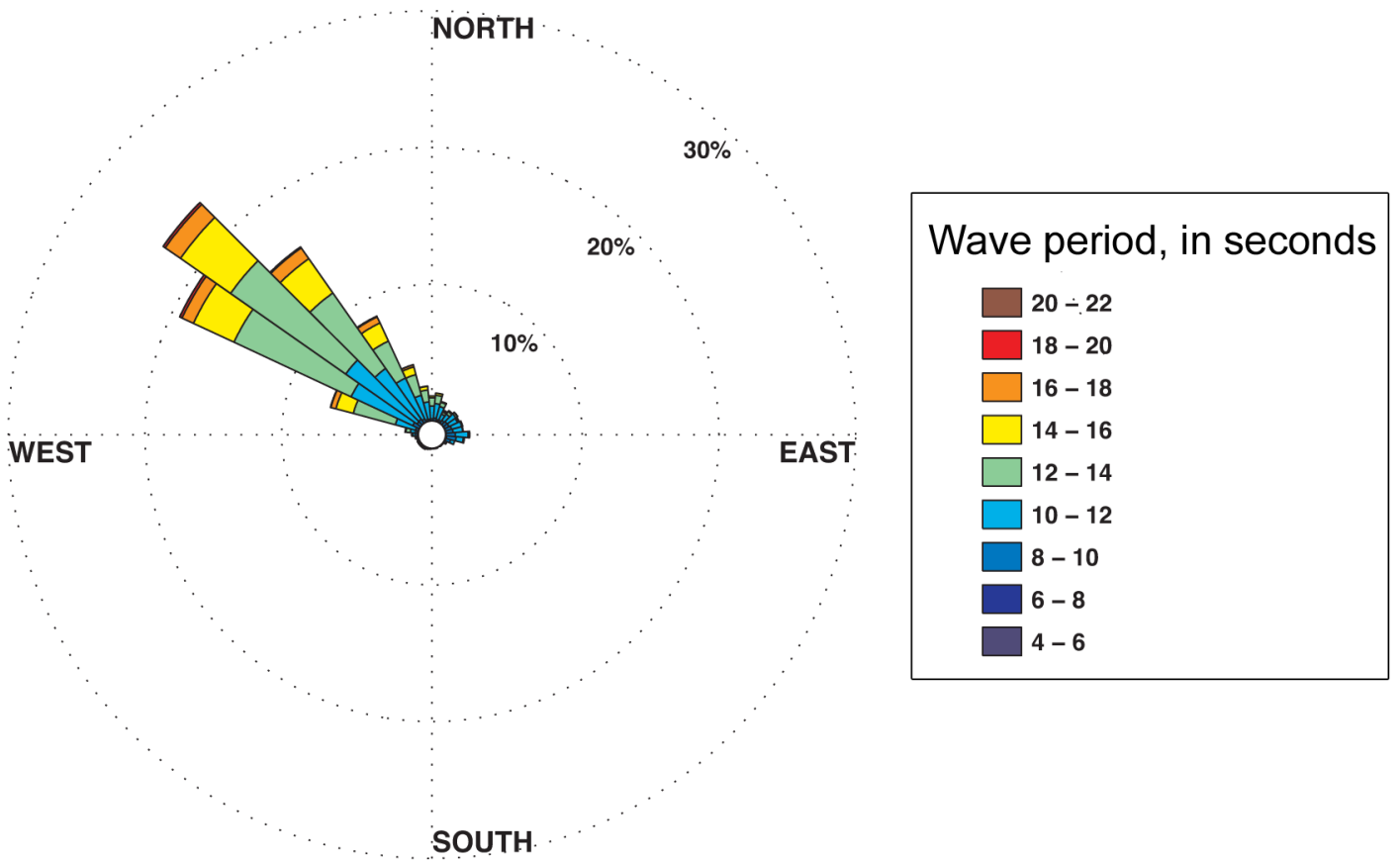

Figure 2.6. Compass plot of variation in wave period in seconds (s), by wave direction (or compass orientation), based on U.S. Army Corps of Engineers (2011) data for the years 1981 to 2004 at a location $\left(28^{\circ} 00^{\prime} \mathrm{N}, 174^{\circ} 00^{\prime} \mathrm{W}\right.$ ) approximately 375 kilometers northwest of Laysan Island. Frequency of occurrence (in percent) increases outward from the center of the compass plot. Wave period data, along with wave height (fig. 2.5) and wind speed (fig. 2.7) data, were used to simulate wave-driven water levels under various sea-level rise scenarios. 


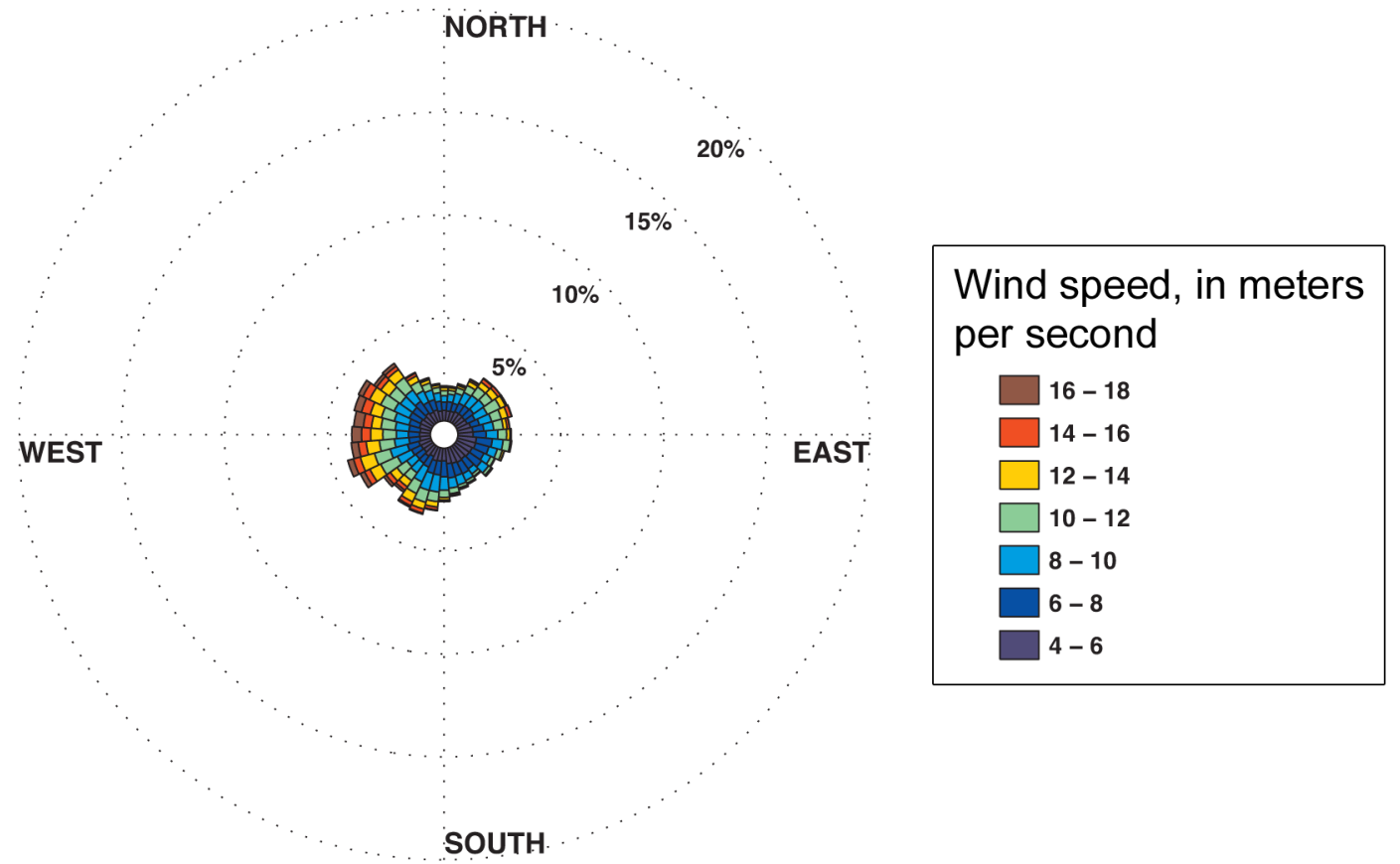

Figure 2.7. Compass plot of variation in wind speed in meters per second $(\mathrm{m} / \mathrm{s})$, by wave direction (or compass orientation), based on U.S. Army Corps of Engineers (2011) data for the years 1981 to 2004 at a location $\left(28^{\circ} 00^{\prime} \mathrm{N}\right.$, $174^{\circ} 00^{\prime} \mathrm{W}$ ) approximately 375 kilometers northwest of Laysan Island. Frequency of occurrence (in percent) increases outward from the center of the compass plot. Wind speed data, along with wave height (fig. 2.5) and period (fig. 2.6) data, were used to simulate wave-driven water levels under various sea-level rise scenarios.

meters and a common datum, we generated two grids using the geostatistical interpolation technique of kriging (Childs, 2004) in ArcGIS 10 (ESRI, 2010). These two grids represent boundary input for the Delft3D modeling software.

\section{Modeled Water Surfaces and Wave Run-up}

We used the Delft3D WAVE module to generate parameters for our wave-driven inundation computations. Similar to other models that estimate water surfaces such as the Sea, Lake and Overland Surges from Hurricanes (SLOSH) model used by the National Hurricane Center (2011) to estimate storm surge, the Delft3D model produces a grid of output parameters over a wide spatial area. For Delft3D, output parameters include wave height, wavelength, and wave-induced set-up (a rise in mean water level inshore of the initial point of wave-breaking). We extracted these parameters for each grid cell for all present and future sea-level scenarios $(0.00,+0.50,+1.00,+1.50$, and $+2.00 \mathrm{~m}$ ), as defined on the basis of studies and projections from the IPCC (2007), Grinsted and others (2010), and Nicholls and Cazenave (2010). Using extracted wave height and wavelength data, we computed wave-induced run-up heights (height above still water levels reached by wave-driven swash) with the methodology of Walton and Ahrens (1989) and Stockdon and others (2006). Then for each cell, we summed SLR values, wave-induced set-up, and run-up heights into total water levels, or elevations of inundation. The method for projecting these water levels up the beach slope is discussed below (see Methods, GIS Analysis).

\section{Coral-Reef Accretion}

Our methodology and bathymetric grids reflect the current state of the reef without future accretion. Coral-reef accretion is the natural biological and sedimentological process of adding material to an existing reef. Potential vertical reef accretion was not included in this study because no data exist for Laysan and published vertical reef-flat accretion rates for reef flats exposed to open-ocean storm waves (1-4 mm/yr according to Buddemeier and Smith, 1988; Montaggioni, 2005) are very small compared with the rates of projected SLR until $2100(8-16 \mathrm{~mm} / \mathrm{yr}$ according to Grinsted and others, 2010; Nicholls and Cazenave, 2010). These vertical accretion rates, which are not equal to coral growth rates, suggest that projected SLR will outpace potential vertical reef-flat accretion, resulting in a net increase in water depth over exposed reef flats on the order of $0.4-1.5$ $\mathrm{m}$ during the 21 st century. At the northern end of the NWHI, where water temperatures are generally cooler and coral reef accretion rates slower, vertical accretion rates are close to zero; Kure Atoll has an accretion rate of $0.2 \mathrm{~mm} / \mathrm{yr}$, compared 


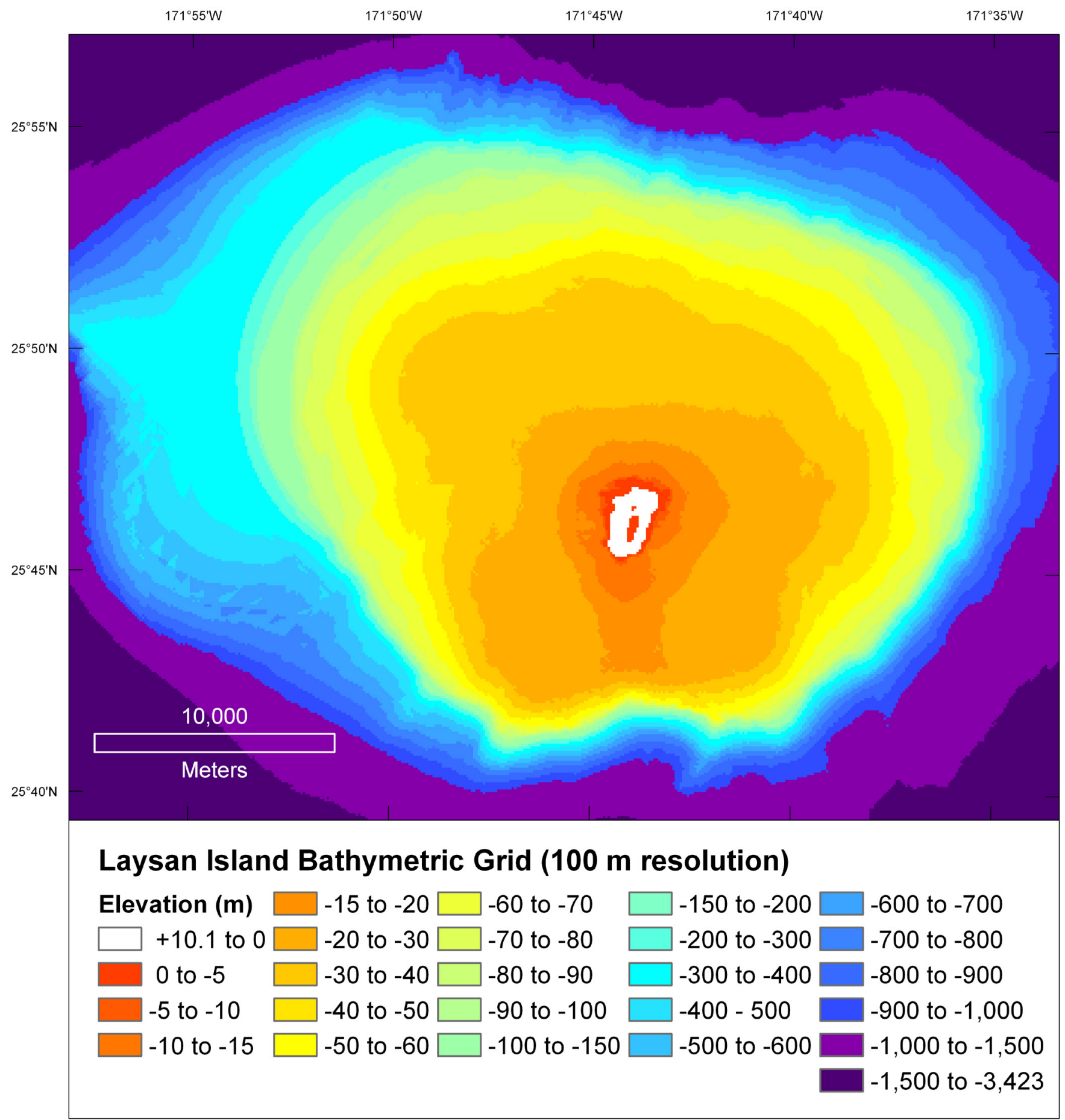

Figure 2.8. Seamless 100-meter $(\mathrm{m})$ resolution bathymetric grid of Laysan Island within Papahānaumokuākea Marine National Monument. Data were compiled from Pacific Island Benthic Habitat Mapping Center (PIBHMC, 2011) bathymetric grids, National Oceanic and Atmospheric Administration Office of Coast Survey (2011) navigational charts, PhotoSat Information Ltd. (2010) digital elevation models, and DigitalGlobe Inc. (2010) WorldView-2 satellite imagery. This compilation represents the coarse input grid to the wave-driven inundation model. 


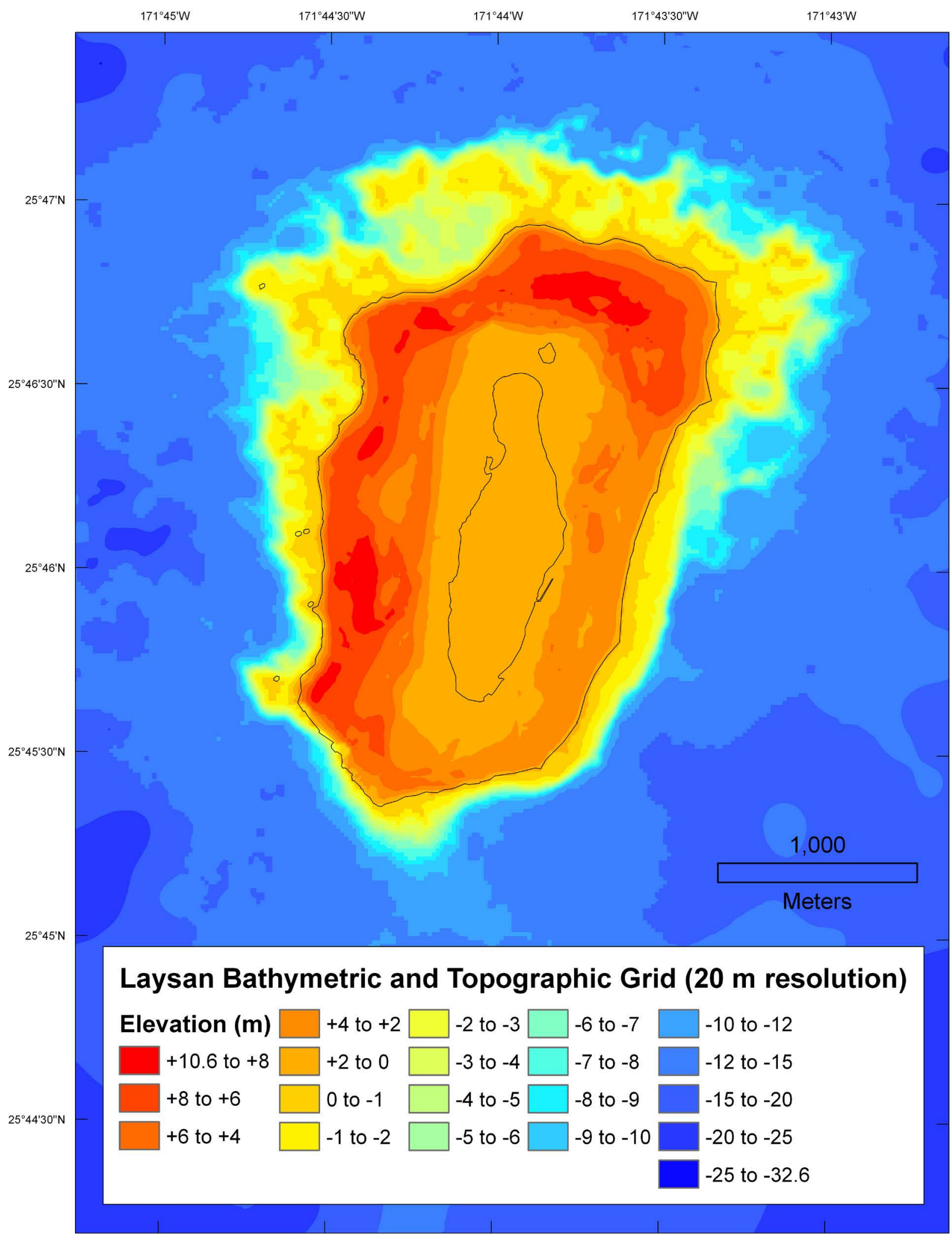

Figure 2.9. Seamless 20-meter $(\mathrm{m})$ resolution bathymetric and topographic grid of Laysan Island, the finer input grid to the wave-driven inundation model. Data were compiled from Pacific Island Benthic Habitat Mapping Center (PIBHMC, 2011) bathymetric grids, National Oceanic and Atmospheric Administration Office of Coast Survey (2011) navigational charts, PhotoSat Information Ltd. (2010) digital elevation models, and DigitalGlobe Inc. (2010) WorldView-2 satellite imagery. 
to long-term accretion rates of $2 \mathrm{~mm} / \mathrm{yr}$ in the wave-sheltered bays of the main Hawaiian Islands (for example, Hanauma and Kāne‘ohe; Grigg, 1998, 2008).

\section{Estimating Mean Sea Level}

Prior to mapping inundation, we delineated the coastline (mean sea level, MSL) based on satellite imagery and predicted tides at the time of the photo (PhotoSat Information Ltd., 2010; NOAA, 2011b). Since no tide gauges or tidal epochs exist for Laysan, the predicted tide at the time of the photo $(+0.064 \mathrm{~m}$ above mean lower low water, MLLW, based on scaled heights from the Honolulu gauge, NOAA Station ID 1612340) was assumed to represent the best estimate of tide at 10:30 a.m. local time, May 18, 2010. Scaled heights from the Honolulu gauge indicate that the predicted tide at the time of the photo would lie $-0.065 \mathrm{~m}$ below predicted MSL (or $+0.129 \mathrm{~m}$ above MLLW, scaled from Honolulu values), while mean high water (MHW) would lie $+0.233 \mathrm{~m}$ above the MLLW mark. During the 24-hour period when satellite photos were taken, the tide gauge at Midway Atoll (NOAA Station ID 1619910) registered only slight differences (mean difference $=0.07 \mathrm{~m}$ over 24 hours) between predicted and verified tides, indicating that no major oceanographic or meteorological phenomena would have affected tidal predictions for Laysan Island. For the purpose of mapping inundation, this analysis used MHW (defined as the average of all high water heights over a 19-year National Tidal Datum Epoch, a period of time that includes most tidal variation due to lunar and solar forces) as the vertical reference level for each SLR and wave-driven inundation scenario; thus we generally modeled the worst-case daily scenario.

\section{GIS Analysis}

The remaining inundation modeling involved a number of sequential steps, primarily carried out in a GIS framework using the North American Datum of 1983 (NAD83) as the horizontal control datum and the Universal Transverse Mercator (UTM) coordinate system, zone $2 \mathrm{~N}$, as the spatial reference. Vertical analysis referenced local sea level (generally MHW) as described above.

First we linked our modeled wave-driven water-level surface (set-up + run-up), which was stored in a 20-m grid format, to the Laysan coastline using nearest neighbor techniques (ESRI, 2010). Nearest water-level values were assigned to coastal points spaced $20 \mathrm{~m}$ apart. We represented the Laysan coast with 430 points, each with a Delft3D wave-driven water-level value. For each SLR scenario, we projected inundation inland from each coastal point along shore-normal transects. Given the highly convoluted nature of the Laysan coastline, we determined shore-normal transects to lie in a direction perpendicular to alongshore coastal segments, rather than perpendicular to the instantaneous orientation at each $20-\mathrm{m}$-spaced coastal station. These coastal segments were drawn as regression lines based on the location of each coastal point and its ten nearest neighbors (five to each side); orthogonal transects were defined to run in the direction of the negative reciprocal of the regression slope. For each scenario, we projected combined SLR and wave-driven water levels orthogonally inland from each coastal point until land elevations exceeded combined water heights, with the highest point reached on each shore-normal transect representing the high-water marks (HWMs). We then delineated the extent of inundation by connecting the HWMs from all 430 transects.

For transects with much longer run-up distances than adjacent transects, we modeled water to flow into adjacent topographic depressions if these depressions were hydrologically connected and at lower elevations than the inundated transects. While the Delft3D model produces wave-driven water levels, it does not estimate water volumes; therefore, the volume of water (reaching the wave-driven water level) that is likely to flow into adjacent topographic depressions is unknown. Another unknown factor is the infiltration capacity of the land, which influences how much water reaches and settles in these basins rather than percolates into the ground. Thus in depicting the extent of inundation for SLR scenarios, we were able to map the initial inundation patterns as wavedriven water moved onshore, representing a transient state before wave-driven seawater volumes flowed laterally into adjacent depressions. We also were able to delineate the maximum extent of inundation if wave-driven water volumes were unlimited and no infiltration occurred. Additional complexities in surface hydrology and island morphology were not considered (see Discussion, Future Research Directions, for potential enhancements to these methods).

For both passive and dynamic modeling approaches, we analyzed and mapped inundation extent within an ArcGIS framework under five potential sea-level scenarios $(0.00$, $+0.50,+1.00,+1.50$, and $+2.00 \mathrm{~m})$. The passive approach represents a reference level and depicts the amount of inundation due to SLR only, while the wave-driven water-level approach considers the additional effect of wave-driven set-up and run-up from the top 5 percent of winter storm events (USACE, 2011). As described above, all inundation maps depict flooding under a worst-case daily scenario at MHW.

\section{Hydrogeology}

No monitoring wells or hydrological data exist for Laysan and the hydraulic conductivity of various geologic formations remains unknown; therefore, the effect of SLR on the water table and consequently lake levels within Laysan's central basin (as well as eastern basins) is uncertain. Precipitation is predicted to decrease in the Hawaiian Islands with climate change (IPCC, 2007), but we did not incorporate this in our scenarios of the lake's projected hydrology. Because no paleoenvironmental evidence exists to indicate that this lake has dried out completely within the past 7,000 years, Athens and others (2007) concluded that some percolation of seawater 
into the lake is likely; however, since tidal fluctuation has not been observed in the lake (Ely and Clapp, 1973), these percolation rates must be low. If some degree of percolation occurs between the ocean and lake, we speculate that over the long-term, lake levels may rise at a similar rate to SLR rates, as observed in hydrological studies on the main Hawaiian Islands (Rotzoll and others, 2007; Gingerich, 2008). If this were to occur, rising groundwater may cause inundation of the low-lying areas adjacent to the lake. Thus, we explored and presented most of our results in two ways: (1) assuming no percolation of seawater to the lake, and (2) assuming sufficient percolation to allow lake levels to rise at similar rates to those of sea level.

\section{Land Cover and Habitat Loss}

We defined and classified land cover for Laysan Island using a combination of USFWS data (Hammond and others, 2010) and remotely sensed data using WorldView-2 satellite imagery (Digital Globe Inc., 2010). Whenever possible, we used ground-survey data (available for four plant species, the hard pan, and wetland area) to delineate land cover; otherwise unsupervised classification methods (whereby computer software executes a clustering process to classify pixels; see chap. 1) were used due to logistical constraints and limited ground-truth data for various habitat/land cover classes currently present on Laysan Island (Xie and others, 2008; see chap. 1 for additional details). In ArcGIS, we developed signature files (characterizing the reflectance of electromagnetic radiation from the earth's surface) for each land cover class using the IsoCluster tool and performed the classification using maximum likelihood methods (ESRI, 2010). Field biologists were consulted to review the land cover categories, as well as to assess the accuracy of our land cover maps (M. Stelmach, USFWS, oral commun., 2011). In general, our land cover classes were similar to the categories used by USFWS field biologists (USFWS data; Hammond and others, 2010). By overlaying predicted inundation patterns with our remotely sensed land cover maps, we quantified the land cover classes likely to be inundated.

The land cover classes consisted of 15 categories of varying specificity (also see chap. 1). Five categories represented broad vegetation classes: tree/shrub, mixed shrub, grass/herbaceous cover, vine/ground cover, and wetland vegetation. As the name implies, wetland vegetation typically occurred outside of the lake-zone mudflats and consisted of areas with wetland plant species (primarily sedges and matted vegetation). Four classes delineated the distribution of individual plant species: ironwood (Casuarina equisetifolia), coconut tree (Cocos nucifera), Indian camphorweed (Pluchea indica), and tree heliotrope (Tournefortia argentea). Three classes differentiated unvegetated areas into bare ground, hard pan, or beach. Beach consisted of the coastal band subject to tidal action and regular wave swash, while bare ground consisted of bare ground inland of the beach zone. Located on a flat, former guano-mining site, the hard pan was composed primarily of guano (Ely and Clapp, 1973). We divided wetland areas (that is, the lake zone) into two categories, depending on whether standing water existed at the time of the satellite image on May 18, 2010 (Digital Globe Inc., 2010). Lastly, we included a category for all human structures on the island.

To estimate habitat-specific losses, we overlaid our land cover classes with five inundation patterns, one from each of the following five modeling approaches: a passive model, a wave-driven inundation model, a wave-driven inundation model with unlimited seawater volumes and no infiltration, a passive model with rising groundwater, and a wave-driven inundation model with rising groundwater. We chose not to analyze the combined effects of wave-driven inundation (with unlimited seawater volumes and no infiltration) and rising groundwater, as these two approaches generally inundated the same low-lying areas, rendering this modeling combination redundant. In presenting our results, we generally expressed area as a percent of total terrestrial area, which included all land cover classes except for unvegetated wetland (that is, lake zone mudflat and standing water) categories.

\section{Nesting Habitat and Distribution}

Using descriptions of breeding bird habitat, we identified all land cover classes potentially used as nesting habitat and conducted analyses to identify SLR vulnerability for nine of the breeding bird species on Laysan Island with data (Blackfooted Albatross, Laysan Albatross, Masked Booby [Sula dactylatra], Brown Booby [S. leucogaster], Red-footed Booby [S. sula], Great Frigatebird [Fregata minor], Sooty Tern [Onychoprion fuscata], Laysan Teal, and Laysan Finch; see tables 1.7 and 1.8). Using existing geospatial data (described below), we overlaid predicted inundation areas on current nesting areas for the nine breeding bird species listed above (USFWS data; Morin and Conant, 2002; USFWS, 2005; Cornett and others, 2008; Boyd and others, 2009; Hammond and others, 2010; Reynolds and others, 2010). We assumed that these data provide an index of the breeding distribution and typical breeding abundance observed under current conditions, although in most cases these assumptions should be tested (Citta and others, 2007; Seavy and Reynolds, 2009; see appendix 3).

For Black-footed Albatross, we determined nesting area distributions from land cover maps, survey data, and 2010 distribution maps (USFWS data; Kristof and others, 2010; see Methods, Breeding Bird Abundance). The Black-footed Albatross nesting area included all suitable habitat types determined by overlaying land cover and survey data. For the Laysan Albatross, nesting area was also determined based on land cover maps, long-term monitoring data, and recent surveys, essentially including suitable habitat types across the island.

For Masked and Brown booby, USFWS biologists mapped a sub-sample of nest sites on June 17-19, 2009 (163 of 190 Masked Booby and 35 of 78 Brown Booby nests), 
recording location (with a Garmin GPS map 76 unit; USFWS data; Boyd and others, 2009). Since standard mean incubation count (MIC) methodology was not used on Laysan to index the abundance of nesting Masked and Brown booby and since both species have a protracted breeding season, we cannot use the data to evaluate population size (Citta and others, 2007;

Seavy and Reynolds, 2009). Despite these data limitations, we assumed the limited sample was a representative index for the purposes of mapping and exploring the vulnerability of these breeding colonies to SLR and wave-driven inundation.

Using similar survey methods, USFWS biologists conducted a survey of Red-footed Booby and Great Frigatebird nests on June 10-12, 2008 (USFWS data; Cornett and others, 2008). Using hundreds of GPS points and track files for these two shrub-nesting species, USFWS staff consolidated these data into 24 nesting colonies (USFWS data; Cornett and others, 2008). Although both species are not truly synchronous nesters (Citta and others, 2007) and some breeders would be missed during a two-day survey, this single sample allowed us to model the vulnerability of the assumed breeding distributions of these species to SLR-induced and wave-driven inundation at Laysan.

Sooty Tern colonies were mapped by USFWS biologists on July 11-12, 2008; July 21, 23, 27-28, 2009; and summer 2010 (USFWS data; Cornett and others, 2008; Boyd and others, 2009; Hammond and others, 2010). During each survey, USFWS crew members walked the perimeter of Sooty Tern colonies, leaving a 5-7 m buffer that was removed during processing. Biologists did not collect density data during these surveys, so Sooty Tern abundance was not estimated. We overlaid inundation scenarios on spatial distributions from the 3 years with colony data.

Habitat studies of Laysan Teal using radio telemetry (Reynolds, 2004; Reynolds and others, 2007) indicated that Laysan Teal use all vegetated habitat types that provide dense cover for nest concealment on Laysan, but they typically avoid some land cover classes while nesting: wetland vegetation, bare ground, hard pan, and beach (table 1.8). Laysan Finch also occupy all vegetated land cover types but primarily use bunch grass for nesting on Laysan Island (Morin and Conant, 2002). Laysan Finch on Laysan Island generally avoid nesting in wetland vegetation, bare ground, hard pan, and beach, but they use vine/ground cover and shrubs for nesting in low densities (Morin and Conant, 2002; table 1.8). For both of these endangered endemics, we used our land cover classes to define potential nesting area (table 1.8) and assumed uniform densities; thus, we are likely to overestimate their nesting habitat.

\section{Nesting Phenology and Temporal Overlap with Storm Season}

To describe species-specific vulnerability, we integrated the seasonal variation and life-history characteristics of breeding birds with wave-energy data showing seasonal variation in mean high-wave and high-wind values from 1981 to 2004 for a location $\left(28^{\circ} 00^{\prime} \mathrm{N}, 174^{\circ} 00^{\prime} \mathrm{W}\right)$ approximately $375 \mathrm{~km}$ northwest of Laysan Island (USACE, 2011). The frequency and magnitude of storm events may increase with SLR (Richardson and others, 2009). We used published literature, field reports, and historical nesting accounts at Laysan Island to develop an island-specific breeding phenology for 20 bird species. To identify periods of adult presence, stage of reproductive investment, and potential risk to the populations, phenology was divided into seven periods with minimum (offpeak) or maximum (peak) number of expected breeders: (1) adults, off-peak; (2) adults, peak; (3) incubation, off-peak; (4) incubation, peak; (5) incubation and chick rearing; (6) chick rearing, peak; and (7) chick rearing, off-peak.

To evaluate relative risks to eggs, chicks, or breeders to storm events, we grouped species according to nesting characteristics. The first group included Procellariiformes (Blackfooted Albatross, Laysan Albatross, Bonin Petrel [Pterodroma hypoleuca], Bulwer's Petrel [Bulweria bulwerii], Wedge-tailed Shearwater [Puffinus pacificus], Christmas Shearwater, and Tristram's Storm-petrel) that nest above ground, in crevices, or in subterranean burrows. These birds lay a single egg and are constrained to a single reproductive attempt (that is, they are not able to relay if their nests are lost; Warham, 1996). The second group consisted of other ground-nesting species (Redtailed Tropicbird [Phaethon rubricauda], Masked Booby, Brown Booby, Gray-backed Tern [Onychoprion lunatus], Sooty Tern, Brown Noddy [Anous stolidus], and Laysan Teal) that nest on the ground, in low vegetation, or under vegetation, and might relay if their nests are lost. The third group of treeand shrub-nesting species (Red-footed Booby, Great Frigatebird, Black Noddy [A. minutus], White Tern [Gygis alba], Laysan Finch, and Nihoa Millerbird) is also more flexible and often able to relay if eggs or chicks are lost.

\section{Breeding Bird Abundance}

For five species with a time series of abundance data (Black-footed Albatross, Laysan Albatross, Red-footed Booby, Laysan Teal, and Laysan Finch), we assumed uniform nest density across all suitable nesting habitats for the purposes of this model. We estimated current carrying capacity and inferred the potential impacts of inundation on population size (Citta and others, 2007; Reynolds and Citta, 2007; Hatfield and others, 2012). For Black-footed and Laysan Albatross, biologists surveyed nests annually on a series of systematic transects (divided into $5 \times 50 \mathrm{~m}$ quadrats, which are rectangular plots used in ecological studies) between the high tide line and lake zone (USFWS data; Kristof and others, 2010). We used albatross data from 2001 through 2011 from quadrats that were counted in all years $(\mathrm{n}=185)$. Red-footed Booby have been surveyed once annually since 1991 (with no data collection in 1996); however, current methods do not implement the standard monitoring protocols needed to account for observation error, variable detection probability, seasonality, or asynchrony of these species (Citta and others, 2007). 
Laysan Teal population abundance has been monitored twice monthly using temporally systematic surveys and randomized start locations since 1991 (USFWS data; Marshall, 1991; Seavy and others, 2009). Since the mid-1980s, biologists have conducted a single annual count of Laysan Finch on 120 strip transects, $91.4 \mathrm{~m}$ long and $5.0 \mathrm{~m}$ wide, located along the edges of randomized grid squares across the island (USFWS data; McClung, 2005). Current Laysan Finch monitoring methods do not account for observation error or variable detection probability.

\section{Wildlife Population Dynamics in Response to SLR}

Our analysis of vulnerability of nesting populations to SLR-induced and wave-driven inundation assumes wildlife may be limited by available habitat and that population dynamics (that is, reproduction, survival, and rate of population growth) may be influenced by SLR and wave-driven inundation. We developed a conceptual model to describe how changes in sea level, global circulation patterns, storm frequency and intensity, and wave-driven water levels may influence habitat availability, island carrying capacity, and avifauna population dynamics at Laysan Island (fig. 2.10).

We used abundance time-series data to quantify population dynamics and estimate the observed carrying capacity and strength of density dependence (that is, when populations are at high numbers and approaching carrying capacity, population size may decline due to a lack of nest sites, overcrowding, and scarce resources). We used a state-space approach to investigate the population dynamics of Black-footed Albatross (2001-2011), Laysan Albatross (2001-2011), Red-footed Booby (1996-2011), Laysan Teal (1993-2010), and Laysan Finch (1983-2011). Using this approach, we estimated mean carrying capacity (the population abundance that the environment sustains, given currently available conditions) for each species. Because density dependence and environmental stochasticity (randomness) produce fluctuations in population abundance, carrying capacity often is described as a "stationary probability distribution," rather than a point equilibrium (Dennis and Constantino, 1988; Dennis and others, 2006). Density-dependent growth was modeled with the Gompertz equation (Edelstein-Keshat, 1988):

where

$$
X t=a+c X t-1+E t
$$

$X t$ is the natural logarithm of the true population size $(N t)$ at time $t$;

$a$ is the rate of population growth (in the absence of density dependence);

$c$ is the strength of density dependence; and

$E t$ is $\sim N(0, \sigma 2)$, the normally distributed unbiased error.

The case where $c=1$ is the density independent model, while $c$ less than 1 implies density dependence, with smaller values of $c$ implying greater density dependence (Staples and others, 2004; Staples and others, 2005; Dennis and others, 2006). Et represents process variation generated by environmental stochasticity. Additionally, observation error was incorporated by assuming:

where

$$
Y_{t}=X_{t}+F_{t}
$$

$F_{t}$ is $\sim N(0, \tau 2)$; and

$\tau$ is the observation error (also called sampling error), as the true population cannot be counted perfectly.

We then analyzed our count data using methods developed by Seavy and others (2009). The program WinBUGS (version 1.4.3, Lunn and others, 2000) was used to provide a Bayesian analysis (Link and Barker, 2010). Where data were sufficient, we summarized the posterior distributions for $\sigma$ (process variation), $\tau$ (sampling error), $a$ (intrinsic rate of increase), and $c$ (strength of density dependence) by their means, standard deviations, medians, and credible intervals. We then calculated carrying capacities $(K)$ and standard deviations using equations 47-51 from Dennis and others (2006).

\section{Uncertainty in SLR Models for Laysan Island}

Three sources of uncertainty affect the vertical accuracy of our modeled inundation levels: (1) uncertainty in tidal elevations; (2) uncertainty in topographic elevations; and (3) uncertainty in wave-driven water levels (set-up + run-up). A robust tidal datum does not exist for Laysan since no tide gauge exists on the island. Thus, we used NOAA's predicted tide $(-0.065 \mathrm{~m}$ relative to MSL) at the moment the stereo-pair images were captured as a reference elevation and defined our MSL tidal datum relative to this level. The accuracy of NOAA's predicted tides at Laysan Island is unknown since these data have not been verified. However, given the small tidal range in the Central Pacific, the relatively flat tidal levels within 6 hours of the time of the photos (less than $0.10 \mathrm{~m}$ range), and the small deviations between predicted and verified tides at Midway Atoll within 12 hours of the photo time $( \pm 0.07 \mathrm{~m})$, we would expect NOAA's predicted tide relative to predicted MSL to be within $0.10 \mathrm{~m}$ of the actual tide (relative to actual MSL). This uncertainty estimate expresses the relative difference between predicted tidal stage and actual tidal stage (that is, predicted tide relative to predicted MSL versus actual tide relative to actual MSL), not the absolute accuracy of the predicted tide relative to an ellipsoid, geoid, or other fixed surface.

We estimated uncertainty in topographic elevation $(0.32 \mathrm{~m})$ using 27 survey-grade (less than 1 centimeter $(\mathrm{cm})$ accuracy) GPS points collected in April 2011 (NOAA, 2011a; chap. 1). Surveyors located survey points in flat, open areas to ensure that horizontal uncertainty did not have a major effect on vertical accuracy since the root-mean-squared error 


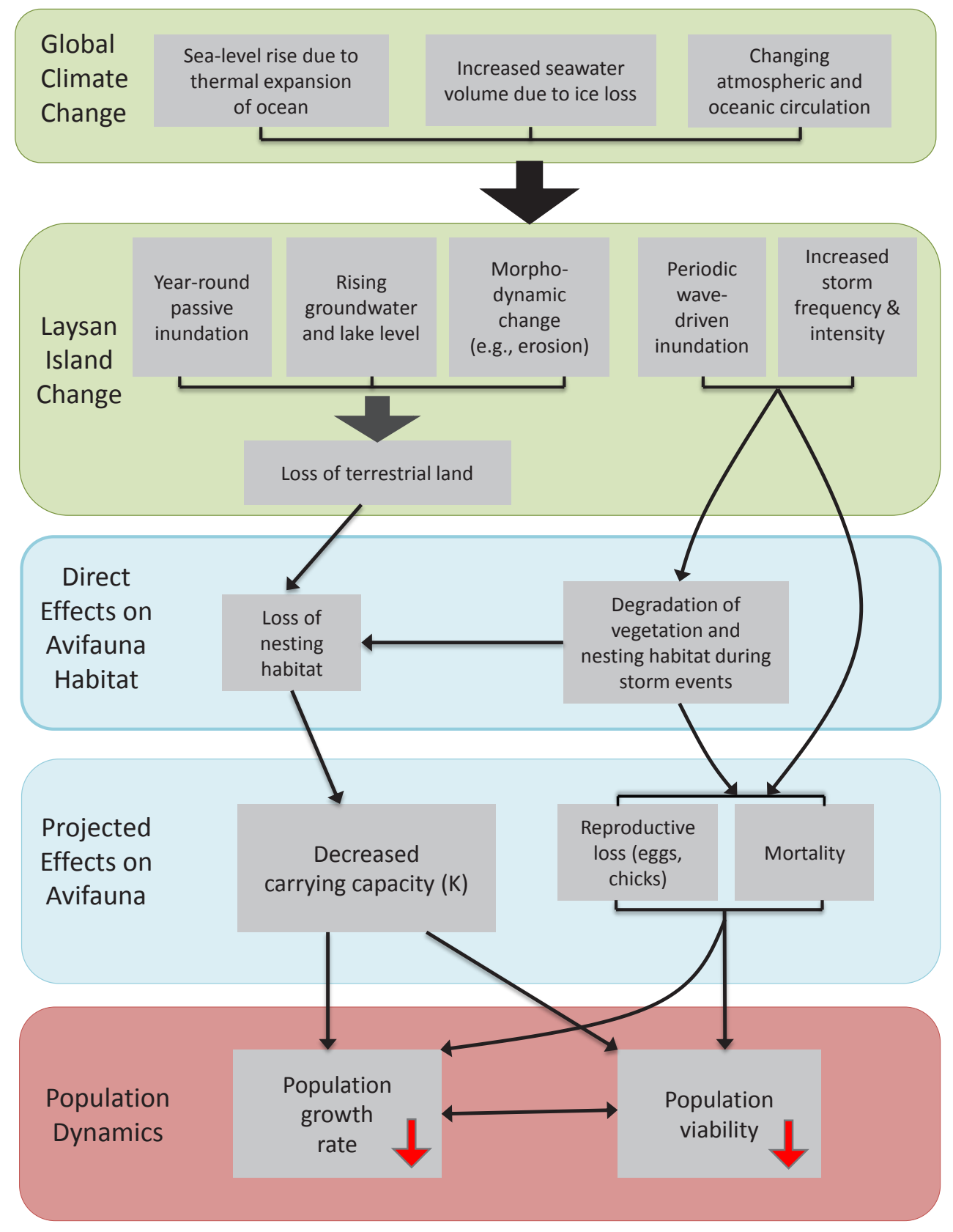

Figure 2.10. Conceptual model describing how global climate change may influence the environment and avifauna of Laysan Island. Thermal expansion of the ocean, increased seawater volume, and changing global circulation patterns are likely to result in a range of impacts specific to Laysan Island. These impacts, in turn, are likely to have direct and indirect effects on avian population dynamics. 
(RMSE) affects our confidence in which areas of the island might be inundated.

Lastly, we conducted sensitivity analysis to address uncertainty in wave-driven water levels and on the input parameters to the wave-driven inundation model. Our analysis indicated that modeled wave height and wave period were less than 2 percent of the mean values for wave height and wave period, with a standard deviation in beach slope of approximately 2 percent. We calculated the resulting mean error in wave-driven water levels as $0.11 \mathrm{~m}$.

Since these uncertainties (expressed as standard deviation, SD) are uncorrelated, we combined them into an overall uncertainty term (NOAA, 2010a):

$$
\begin{aligned}
\mathrm{SD}_{\text {Total }}= & \left(\mathrm{SD}_{\text {Tidal }}{ }^{2}+\mathrm{SD}_{\text {Topographic }}{ }^{2}+\mathrm{SD}_{\text {Run-up }}{ }^{2}\right)^{0.5}= \\
& \left(0.10^{2}+0.32^{2}+0.11^{2}\right)^{0.5}
\end{aligned}
$$

From the uncertainty estimates above, we computed the overall vertical uncertainty or RMSE as $0.35 \mathrm{~m}$. This vertical uncertainty has a variable effect on horizontal inundation extent, depending on topography; areas with gradual slopes exhibit more horizontal uncertainty than areas with steep slopes.

Inundation extents (or boundaries) can be created from data of any quality and are not sensitive to accuracy, although data accuracy determines the area of confidence around inundation boundaries (NOAA, 2010a). NOAA uses a value of 80-percent confidence as the threshold between high and low confidence. By definition, the $Z$-score at the inundation boundary equals zero, with the probability of inundation on this boundary equaling 50 percent. At elevations above the inundation boundary, the probability of inundation decreases according to the normal probability distribution, such that a location +0.84 SDs above the inundation level has a 20 -percent probability of inundation (or 80-percent probability of remaining dry). Similarly, a location -0.84 SDs below the inundation level has an 80-percent probability of inundation (or 20-percent probability of remaining dry). Between these two thresholds lies an area of low confidence (less than 80 percent), while beyond these thresholds are areas of high confidence (greater than 80 percent).

As an example, consider a SLR scenario of $+1.00 \mathrm{~m}$ and a RMSE of $0.35 \mathrm{~m}$. At the inundation boundary (that is, at an elevation of $+1.00 \mathrm{~m}$ ), we observe a 50 -percent probability of inundation. For an elevation of $+0.71 \mathrm{~m}(Z=-0.84 \mathrm{SDs})$, we are highly confident ( 80 percent) that this elevation will experience flooding; likewise for an elevation of $+1.29 \mathrm{~m}(Z=$ +0.84 SDs) we are highly confident this elevation will remain dry. Between 0.71 and $1.29 \mathrm{~m}$ lies an area of low (less than 80 percent) confidence.

None of the projected inundation boundaries were biased by positional uncertainty, although the true position of the boundary may have been higher or lower given the uncertainty in the model. For all sea-level scenarios under consideration $(0.00,+0.50,+1.00,+1.50$, and $+2.00 \mathrm{~m})$, if we wanted to maintain 80 -percent confidence that the inundation boundary would remain dry, then as a precaution we would have had to add $+0.29 \mathrm{~m}$ of elevation to the flood surface and allowed the horizontal boundary to adjust accordingly. While we could have adopted such a precautionary approach, we decided to analyze inundation extent using unbiased inundation boundaries only. Since we modeled multiple scenarios of SLR and presented a range of inundation boundaries, the effects of uncertainty on inundation extent can be visualized by examining changes in inundation extent at different increments of SLR.

\section{Limitations of Wildlife Data}

Wildlife distribution and abundance data from Laysan have many limitations. First, most of our assembled distribution data was collected during a single year. In such cases, we assumed surveys occurred at the peak of the breeding season and represented typical distribution patterns for the species surveyed. For the five species with USFWS nesting distribution maps, GPS (Garmin GPS map 76 or $76 \mathrm{CS}$ ) accuracy was generally within $10 \mathrm{~m}$ (RMSE) for all point locations (Masked and Brown booby), perimeter lines around colonies (Sooty Tern), or mixed data that combined points and track lines (Red-footed Booby and Great Frigatebird). For species whose distribution was estimated from land cover maps, long-term monitoring data, and recent surveys, the accuracy of our land cover map represented the greatest source of uncertainty. Due to logistical constraints, we could not perform a quantitative ground-based accuracy assessment on these land cover maps; however, Laysan field biologists reviewed the map qualitatively (M. Stelmach, USFWS, oral commun., 2011). As was the case with distribution data, we assumed abundance data were collected at the peak of the breeding season and represented a typical year in breeding abundance. Most species with time-series data from Laysan Island had large year-to-year variance, and monitoring methods prevented analyses to differentiate between sampling error and true population fluctuations, often referred to as process variation, or $\sigma$ (Citta and others, 2007; Seavy and Reynolds, 2009).

\section{Results}

Depending on the SLR scenario considered and the modeling approach used, the potential physical and biological impacts of SLR on Laysan Island varied widely, with vulnerability ranging from negligible to significant.

\section{Sea-Level Rise and Wave-Driven Water Levels}

Raw wave-driven water levels (set-up + run-up) from the Delft3D WAVE module are shown in figure 2.11 for two of the five SLR scenarios considered. Values represent vertical water levels at the shoreline before they are projected up the beach slope. Higher wave-driven water levels occurred for the $+2.00 \mathrm{~m}$ 
scenario on all coastlines, regardless of exposure. Mean wavedriven water levels under the $+2.00 \mathrm{~m}$ SLR scenario (fig. 2.11) exceeded wave-driven water levels under the $+1.50 \mathrm{~m}$ scenario (not shown) by about 5 percent. In general, mean wave-driven water levels ranged from $+0.98-1.22 \mathrm{~m}$ depending on SLR scenario, with higher wave-driven water levels occurring at higher SLR scenarios. As expected, for each SLR scenario, higher wave-driven water levels generally occurred on western exposures during the period of highest wave energy (Dec.-Feb.).

\section{Passive and Dynamic Inundation}

Across all sea-level scenarios $(0.00,+0.50,+1.00,+$ 1.50 , and $+2.00 \mathrm{~m}$ ), the extent of inundation predicted under the passive modeling approach covered less area than the inundation extent forecasted by the dynamic modeling approach, particularly when considering unlimited volumes of wavedriven seawater and no infiltration (table 2.2, figs. 2.12-14). Under the passive modeling approach, each of the five SLR scenarios yielded a similar spatial pattern, with inundation progressing inland from the coast in the shape of concentric rings (fig. 2.12). Under this approach, none of the coastal dunes (which have heights of greater than $2.5 \mathrm{~m}$ above MSL) separating the ocean from the interior lake and mudflats were breached by seawater. As sea level increased, inundation area expanded more-or-less linearly, accelerating only slightly at the higher sea-level values (table 2.2, fig. 2.14). For all passive SLR scenarios, inundation was less than 5 percent of the island's terrestrial area.
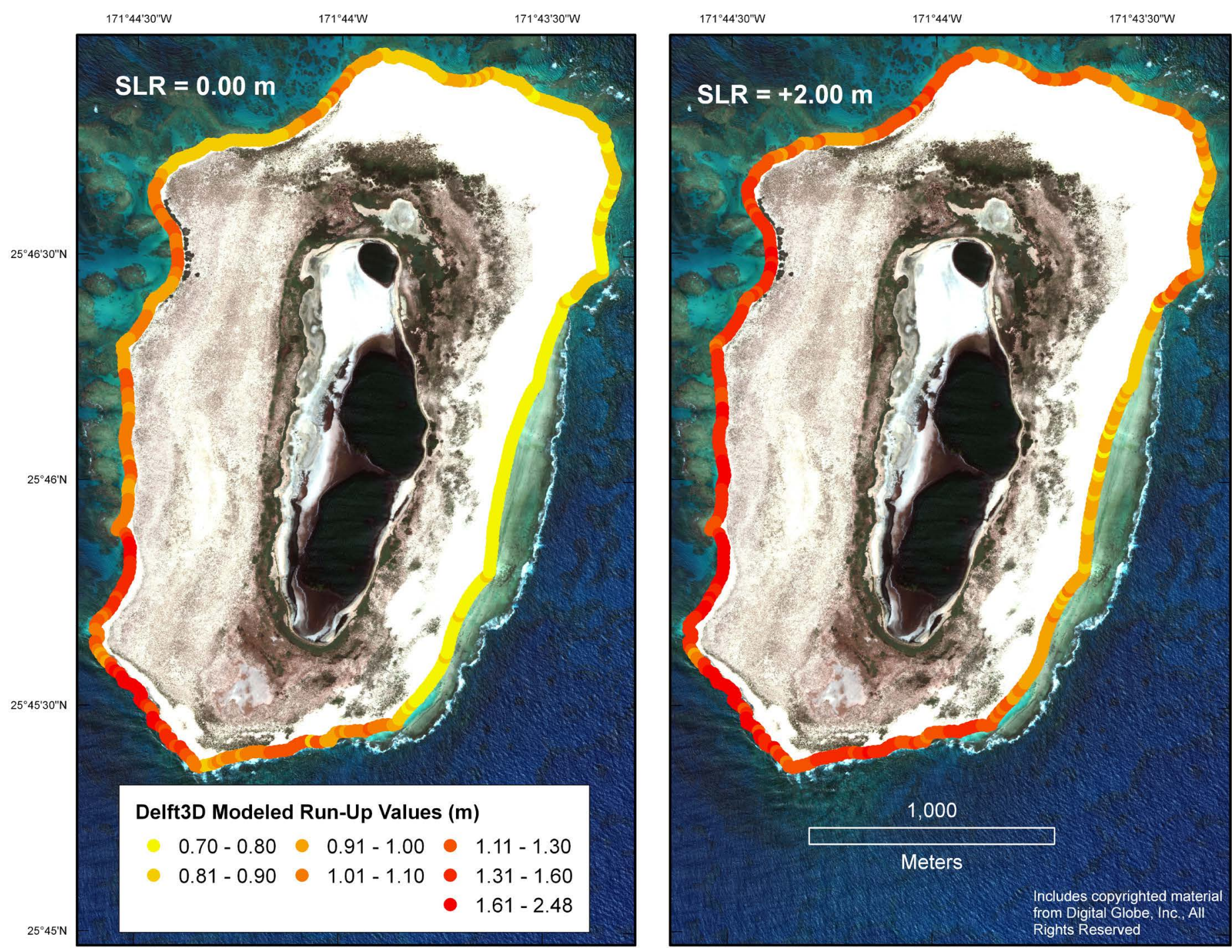

Figure 2.11. Map of Laysan Island depicting modeled wave-driven water levels at 430 coastal points, spaced 20 meters $(\mathrm{m})$ apart, for the lowest and highest sea-level rise (SLR) scenarios: $0.00 \mathrm{~m}$ (left) and $+2.00 \mathrm{~m}$ (right). Wavedriven water levels on the right exhibited higher values, as less wave-energy attenuation occurred across the reef crest and reef flat under higher sea-level conditions. These heights, which include both wave set-up and wave run-up, were projected up shore-normal transects to estimate inundation extent during large-wave events. 
For the dynamic inundation model (fig. 2.13), where SLR was combined with wave set-up and run-up, similar patterns of concentric inundation occurred at SLR scenarios less than or equal to $+1.00 \mathrm{~m}$. For these scenarios $(\mathrm{SLR}=0.00,+0.50$ and $+1.00 \mathrm{~m}$ ), the wave-driven inundation model showed slightly wider bands of inundation than the passive inundation model, although none of the predicted inundation extents exceeded 5 percent of the island's terrestrial area (table 2.2), defined as total island area (412.0 ha) minus the entire lake zone (74.2 ha).

For the higher SLR scenarios of +1.50 and $+2.00 \mathrm{~m}$ (figs. 2.13, 15-16), wave-driven inundation patterns started to diverge considerably from passive inundation patterns, as wave-driven water levels began to breach the coastal dunes separating the ocean from the interior basins (that is, the central lake basin and the two depressions in the eastern desert). At $+1.50 \mathrm{~m}$ SLR, the added effect of wave-driven water levels breached the dunes in two locations along the east coast, including one location where wave-driven water levels penetrated to the lake zone (fig. 2.13). The inundated area from these two breaches intersected the two topographic depressions flooded by the storm event of February 2011 (figs. 2.13 and 2.17; Kristof and others, 2011). In terms of area, at $+1.50 \mathrm{~m} \mathrm{SLR}$ (fig. 2.15), the dynamic wave-driven inundation model (with limited seawater volumes and no infiltration) predicted losses in total terrestrial area of 22.1 ha (6.5 percent of the total terrestrial area), or more than double that from the passive approach (10.8 ha, or 3.2 percent). At $+2.00 \mathrm{~m}$ SLR, the added effect of wave-driven water levels was more dramatic, as seawater propagating from the southern and eastern shorelines penetrated wide expanses of the lake zone. For the +2.00-m SLR scenario (fig. 2.16), the dynamic wave-driven model (with limited seawater volumes and no infiltration) predicted losses in terrestrial area of 58.0 ha (17.2 percent of the terrestrial area), or greater than three times the inundation extent forecasted with the passive modeling approach (15.5 ha or 4.6 percent of terrestrial area).

For the dynamic wave-driven inundation model at the lower SLR scenarios (+1.00 m or less), we observed no differences between initial wave-driven inundation and the theoretical maximum extent of inundation, assuming unlimited seawater volumes and no infiltration. This equality occurred since wave-driven water levels did not overtop the coastal dunes and penetrate to the low-lying interior areas. Once SLR reached $+1.50 \mathrm{~m}$, wave-driven inundation breached the dunes, allowing for greatly increased potential inundation, assuming unlimited volumes of wave-driven seawater and no infiltration. Under these assumptions, the theoretical maximum extent of inundation at $+1.50 \mathrm{~m}$ SLR was 115.8 ha, representing 34.3 percent of the island's terrestrial area (figs. 2.15 and 2.18); at $+2.00 \mathrm{~m}$ SLR, the theoretical maximum extent of inundation covered 136.2 ha, or 40.3 percent of the island's terrestrial area (figs. 2.16 and 2.18). When compared to the observed flooding during the severe winter storm of February 2011

(fig. 2.17; USFWS data; Kristof and others, 2011), our predicted maximum areas of inundation aligned closely (fig. 2.18).

When we incorporated a concurrent rise in groundwater into the passive SLR model, inundation extents expanded considerably, especially in the area adjacent to the lake zone (fig. 2.19). For this model, the narrow band of passive coastal flooding was accompanied by a broad range of interior flooding depending on the SLR scenario considered. At the higher levels of SLR $(+1.50$ and $+2.00 \mathrm{~m})$, extensive inundation of the low-lying lake basin and eastern desert basins occurred in a pattern similar to the one observed for

Table 2.2. Inundation extent for five sea-level scenarios and three modeling approaches: (a) passive inundation model showing inundation due to sea-level rise (SLR) only, (b) wave-driven inundation model showing inundation due to SLR and wave-driven water levels, and (c) wave-driven inundation model depicting inundation due to SLR and wavedriven water levels assuming unlimited seawater volumes and no infiltration of filled basins.

[All values reflect inundation at mean high water (MHW) for the specified SLR scenario. For example, at present sea level (SLR $=0.00 \mathrm{~m})$, inundation represents the area above mean sea level that is submerged at MHW. Percentages represent the percent change in terrestrial land, where terrestrial land (337.8 ha) equals total land area (412.0 ha) minus the two wetland (or lake zone) categories (74.2 ha). By definition, percent change due to inundation is negative, representing a reduction in terrestrial land. Abbreviations: ha, hectares; $\mathrm{m}$, meters; $\%$, percent]

\begin{tabular}{|c|c|c|c|c|c|c|}
\hline \multirow[b]{2}{*}{$\begin{array}{c}\text { SLR } \\
\text { scenario }\end{array}$} & \multicolumn{2}{|c|}{$\begin{array}{l}\text { Passive inundation } \\
\text { due to SLR only }\end{array}$} & \multicolumn{2}{|c|}{$\begin{array}{l}\text { Dynamic inundation } \\
\text { due to SLR and } \\
\text { wave-driven water levels }\end{array}$} & \multicolumn{2}{|c|}{$\begin{array}{c}\text { Dynamic inundation } \\
\text { due to SLR and } \\
\text { wave-driven water levels, assuming } \\
\text { unlimited seawater volumes and no } \\
\text { infiltration }\end{array}$} \\
\hline & $\begin{array}{l}\text { Area } \\
\text { (ha) }\end{array}$ & $\begin{array}{c}\% \\
\text { change }\end{array}$ & $\begin{array}{l}\text { Area } \\
\text { (ha) }\end{array}$ & $\begin{array}{c}\% \\
\text { change }\end{array}$ & $\begin{array}{l}\text { Area } \\
\text { (ha) }\end{array}$ & $\begin{array}{c}\% \\
\text { change }\end{array}$ \\
\hline $0.00 \mathrm{~m}$ & 0.4 & -0.1 & 6.1 & -1.8 & 6.1 & -1.8 \\
\hline$+0.50 \mathrm{~m}$ & 3.1 & -0.9 & 10.5 & -3.1 & 10.5 & -3.1 \\
\hline$+1.00 \mathrm{~m}$ & 6.7 & -2.0 & 15.5 & -4.6 & 15.5 & -4.6 \\
\hline$+1.50 \mathrm{~m}$ & 10.8 & -3.2 & 22.1 & -6.5 & 115.8 & -34.3 \\
\hline$+2.00 \mathrm{~m}$ & 15.5 & -4.6 & 58.0 & -17.2 & 136.2 & -40.3 \\
\hline
\end{tabular}




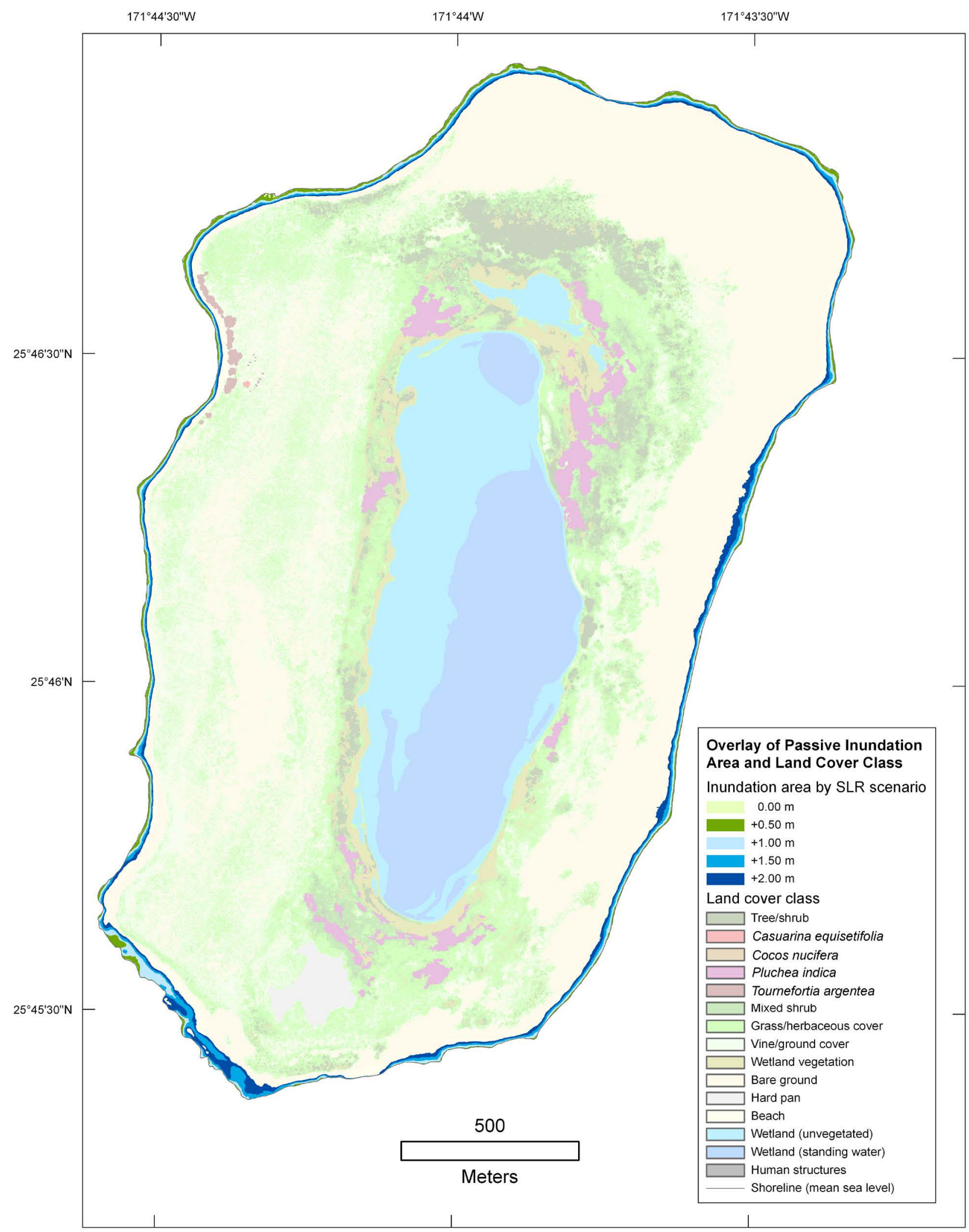

Figure 2.12. Overlay of passive inundation zones for five sea-level rise (SLR) scenarios at Laysan Island with land cover in the background (fig. 2.21 provides a clearer depiction of land cover). This map does not depict wave-driven inundation or inundation from groundwater percolation from the ocean. In general, under this modeling approach inundation occurs only along a narrow stretch of beach around the entire island. All areas represent inundation at mean high water. Abbreviations: $\mathrm{m}$, meters. 


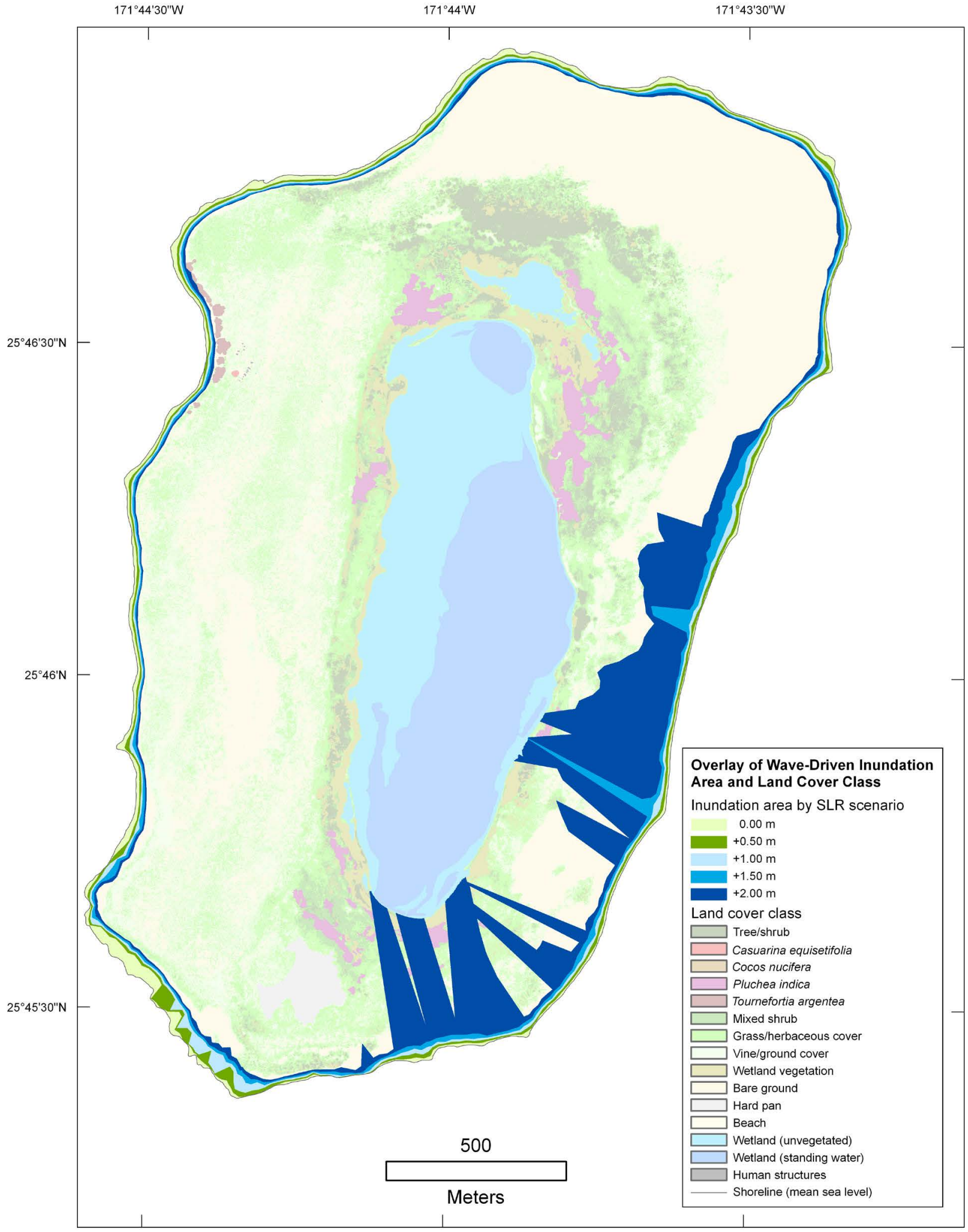

Figure 2.13. Overlay of wave-driven inundation for five sea-level rise (SLR) scenarios at Laysan Island with land cover in the background (fig. 2.21 provides a clearer depiction of land cover). This map does not depict inundation from unlimited volumes of wave-driven seawater, or inundation from groundwater percolation from the ocean. In general, under this modeling approach inundation occurs in wider bands along the beach, with major inland excursions along the south and eastern sides of the island under the higher SLR scenarios (+1.50 and +2.00 meters). All areas represent inundation at mean high water. Abbreviations: $\mathrm{m}$, meters. 


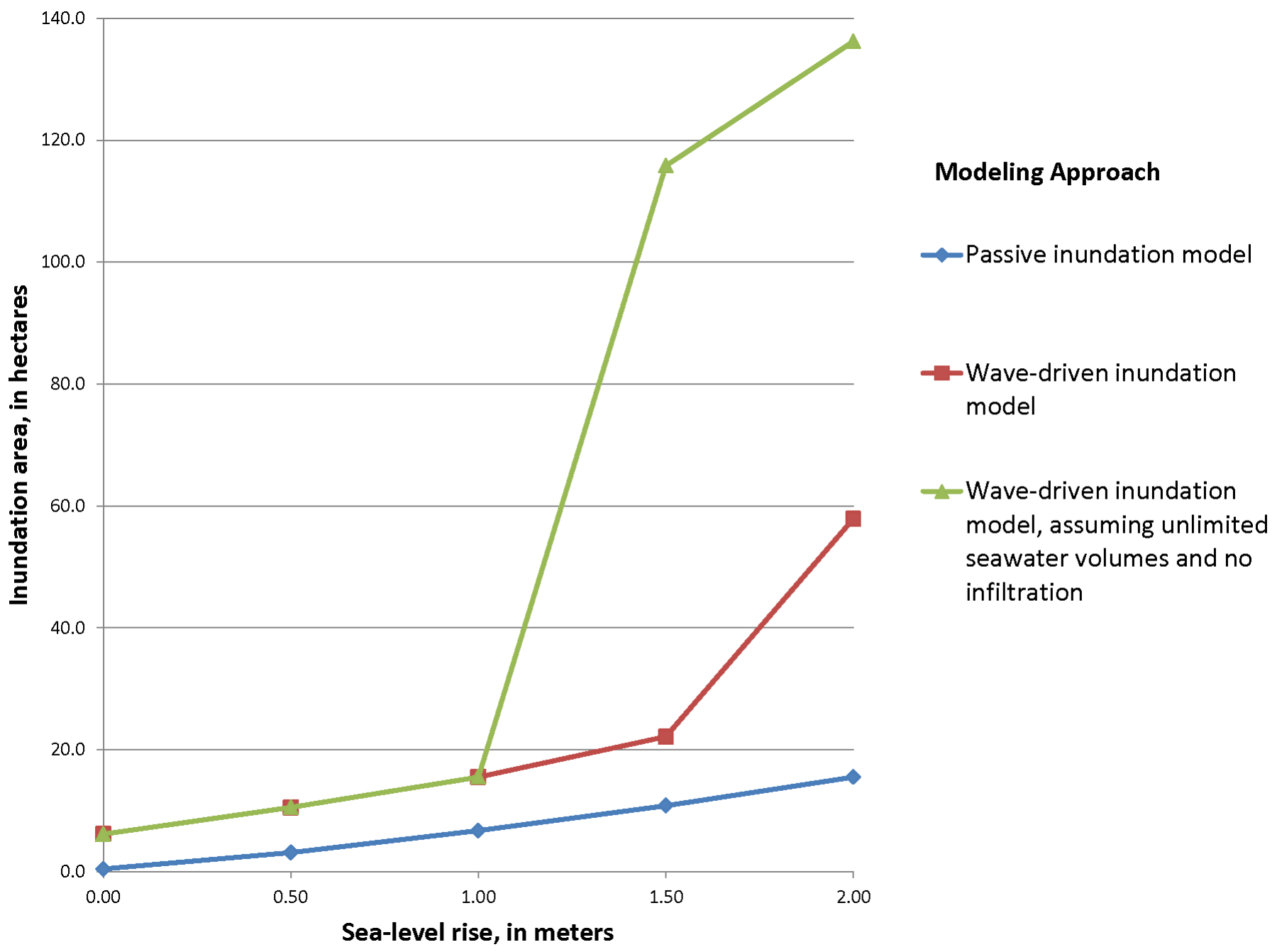

Figure 2.14. Chart of inundation extent in hectares (ha) at Laysan Island for five sea-level rise (SLR) scenarios and three different modeling approaches: (a) passive inundation model (also referred to as a "bathtub model") showing inundation due to SLR only, (b) wave-driven inundation model showing inundation due to SLR and wave-driven water levels, and (c) wave-driven inundation model depicting inundation due to SLR and wave-driven water levels assuming unlimited seawater volumes and no infiltration (see table 2.2). All values reflect inundation at mean high water. For a discussion of the uncertainty associated with the modeling approaches, see Methods (Uncertainty in SLR Models for Laysan Island). 


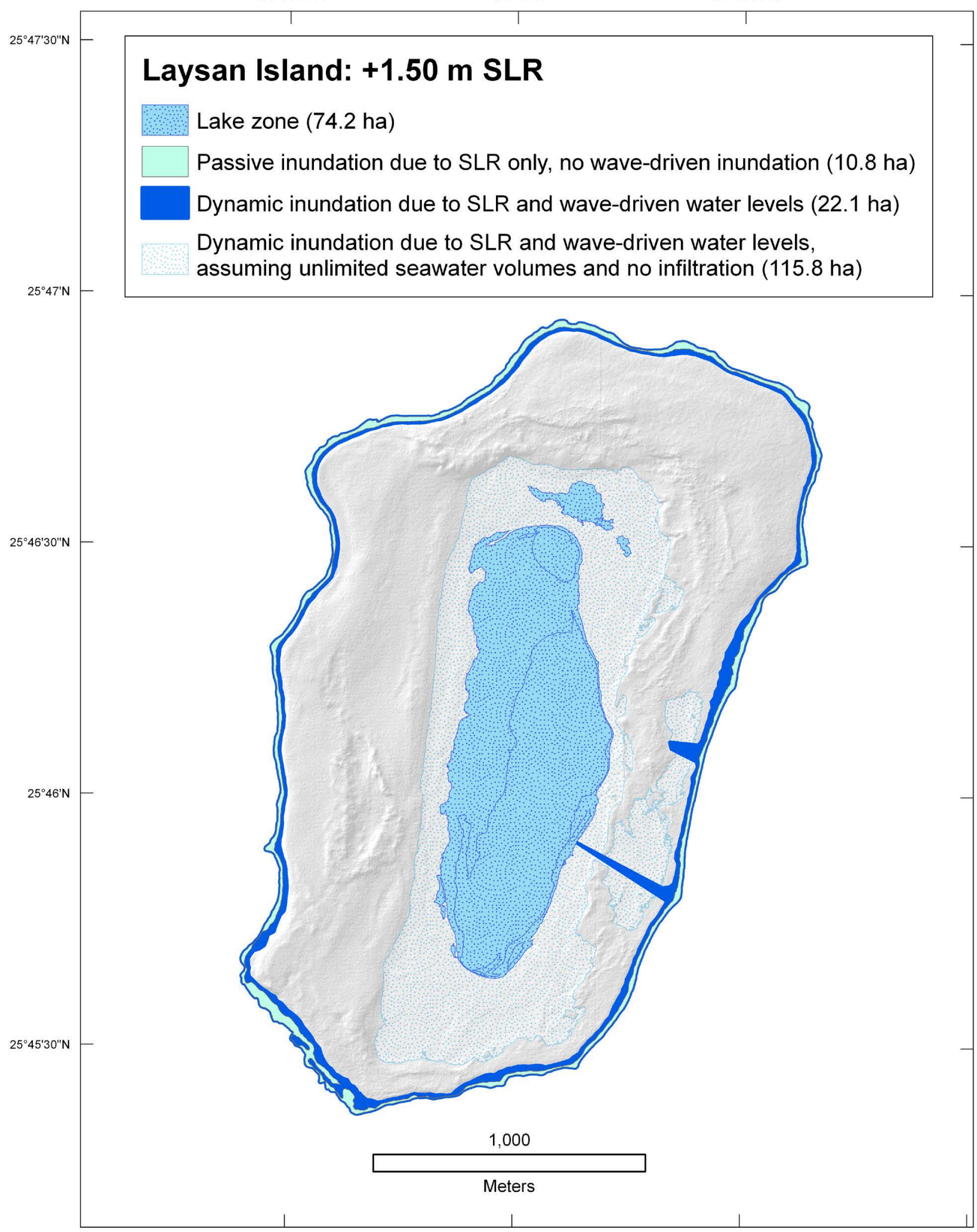

Figure 2.15. Map depicting potential inundation extent at Laysan Island under a sea-level rise (SLR) scenario of +1.50 meters $(\mathrm{m})$ at mean high water. Relative to the passive inundation modeling approach, the dynamic inundation model that includes wave-driven water levels shows a wider band of coastal inundation, plus two locations in the east where inundation extends more than $400 \mathrm{~m}$ inland. If wave-driven seawater volumes were unlimited and no infiltration occurred, an area of 115.8 hectares (ha) could be inundated. 


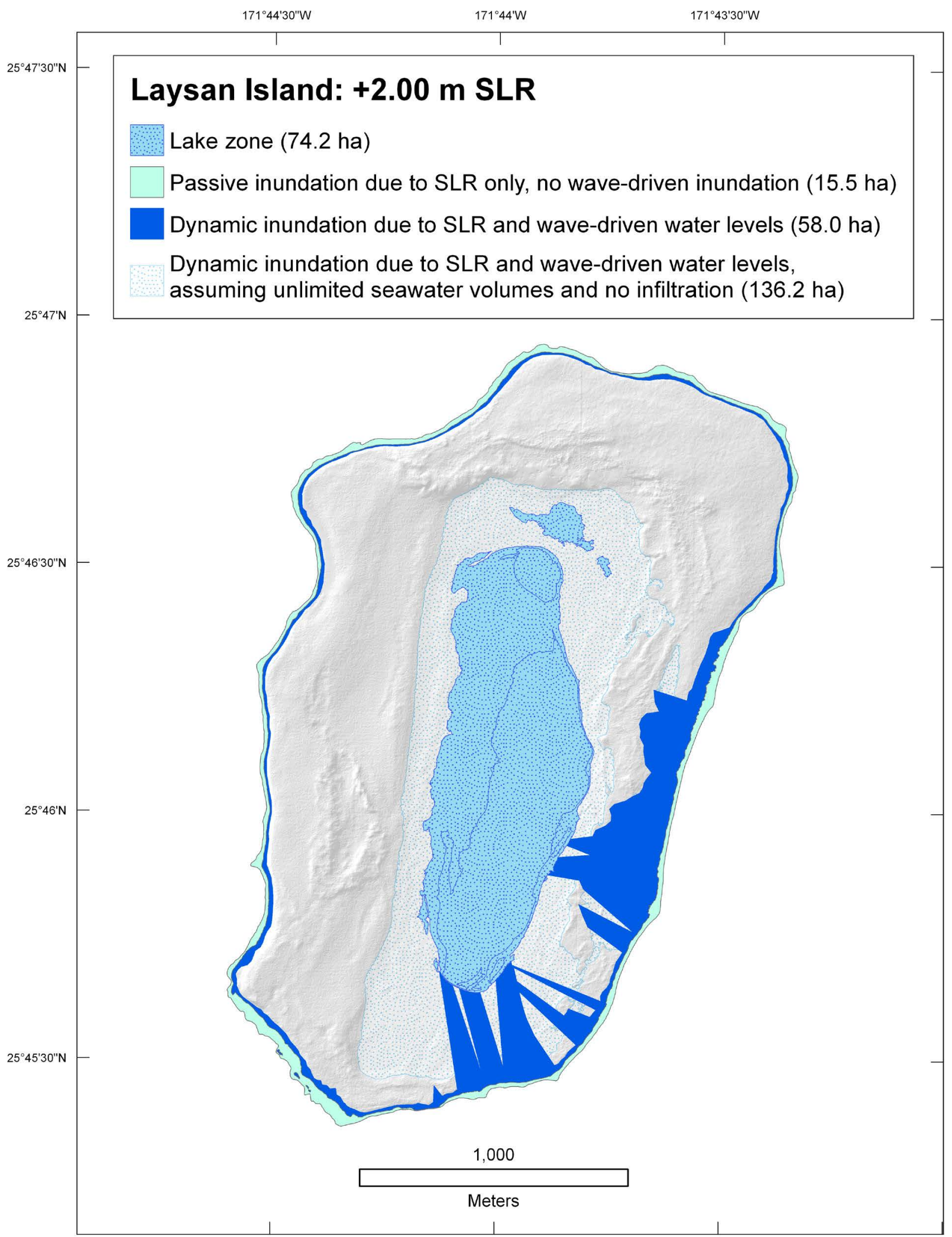

Figure 2.16. Map depicting potential inundation extent at Laysan Island under a sea-level rise (SLR) scenario of +2.00 meters $(\mathrm{m})$ at mean high water. Relative to the passive inundation modeling approach, the dynamic inundation model that includes wave-driven water levels (medium blue) shows a wider band of coastal inundation, plus multiple locations in the east where inundation extends more than $400 \mathrm{~m}$ inland. If wave-driven seawater volumes were unlimited and no infiltration occurred, an area of 136.2 ha (hectares) could be inundated. 


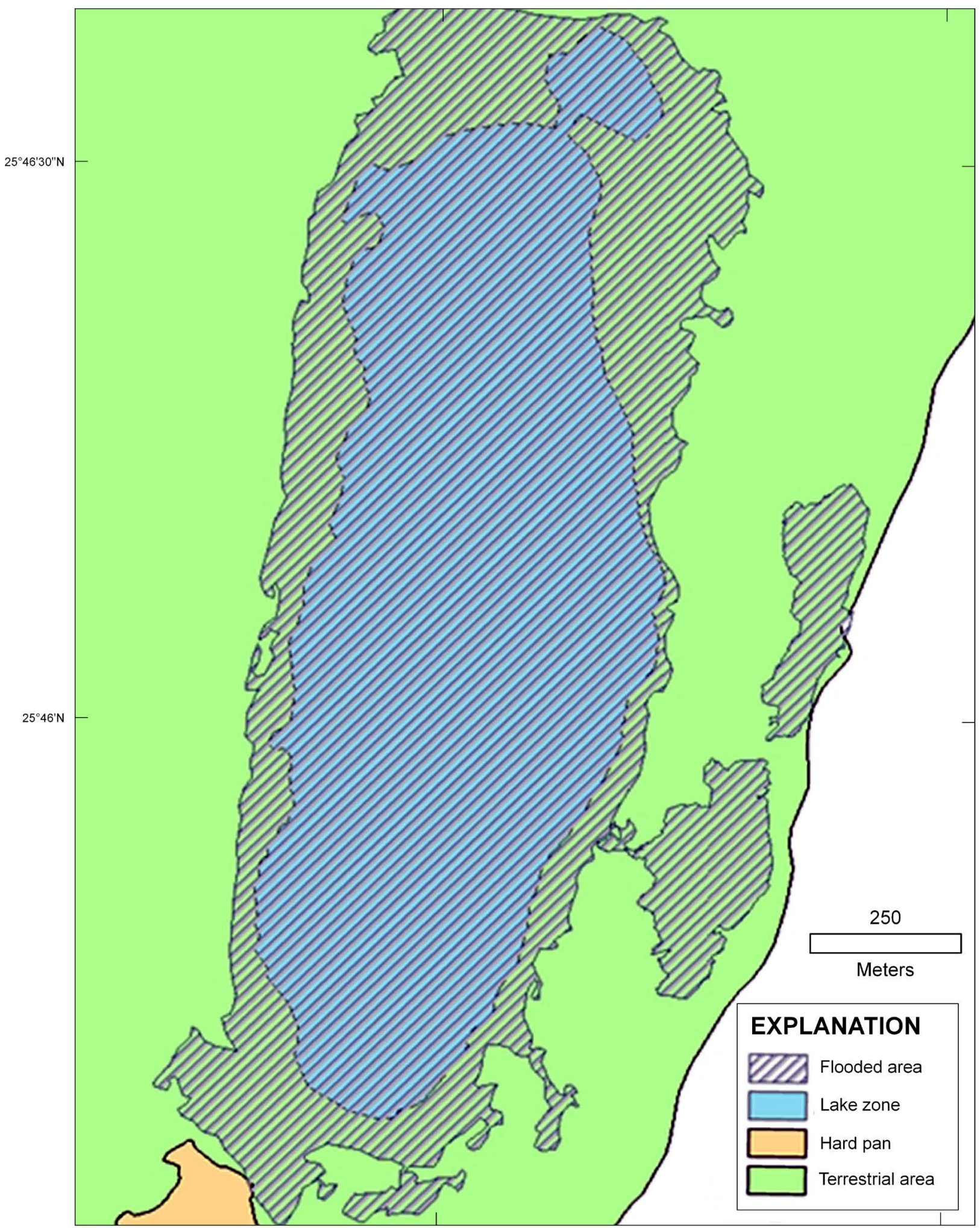

Figure 2.17. The inundation pattern documented by U.S. Fish and Wildlife Service (USFWS) field staff on Laysan Island after a heavy rainfall (27.20 centimeters in 24 hours) and large-wave event on February 12, 2011 (USFWS data; Kristof and others, 2011). The "average" lake zone identified in this USFWS map represents the entire lake zone. Typically this lake zone encompasses areas of standing water and mud flats, depending on recent hydrologic events (for example, heavy rainfall or drought). 


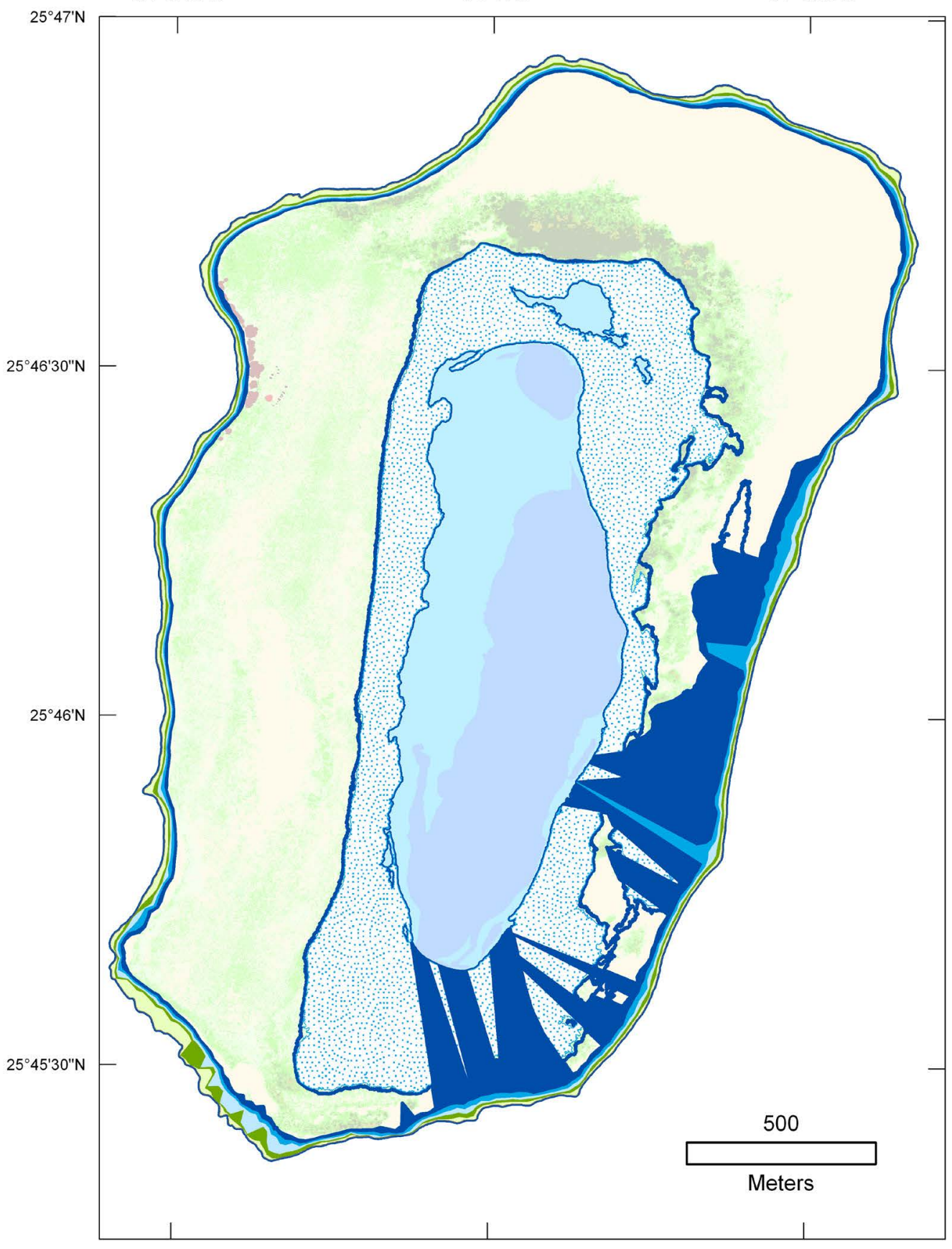

Overlay of Wave-Driven Inundation and Land Cover Class, Including Scenarios with Unlimited Seawater Volumes and No Infiltration Inundation area by SLR scenario

\begin{tabular}{l}
$0.00 \mathrm{~m}$ \\
$+0.50 \mathrm{~m}$ \\
$+1.00 \mathrm{~m}$ \\
$+1.50 \mathrm{~m}$ \\
$+2.00 \mathrm{~m}$ \\
Max theoretical inundation ( \\
Max theoretical inundation ( \\
\hline$\square$ cover class \\
Tree/shrub \\
Casuarina equisetifolia \\
Cocos nucifera \\
Pluchea indica \\
Tournefortia argentea \\
Mixed shrub \\
Grass/herbaceous cover \\
Vine/ground cover \\
Wetland vegetation \\
Bare ground \\
Hard pan \\
Beach \\
Wetland (unvegetated) \\
Wetland (standing water) \\
Human structures \\
Shoreline (mean sea level)
\end{tabular}

Figure 2.18. Overlay of wave-driven inundation extents on Laysan Island assuming unlimited seawater volumes and no infiltration for five sea-level rise (SLR) scenarios, with land cover in the background (fig. 2.21 provides a clearer depiction of land cover). This pattern depicts the theoretical maximum extent of inundation during periods of high-wave energy. It does not show inundation due to groundwater percolation from the ocean. In comparison to the wave-driven inundation model with limited wave-driven seawater volumes (fig. 2.13), this model exhibits extensive inundation around the interior lake and in the eastern depressions for the SLR scenarios of +1.50 and +2.00 meters $(\mathrm{m})$. At the lower SLR scenarios $(0.00,+0.50$, and $+1.00 \mathrm{~m})$, inundation is the same regardless of whether seawater volumes are limited or unlimited, as wave-driven seawater does not overtop the coastal dunes and penetrate to the interior basin. 


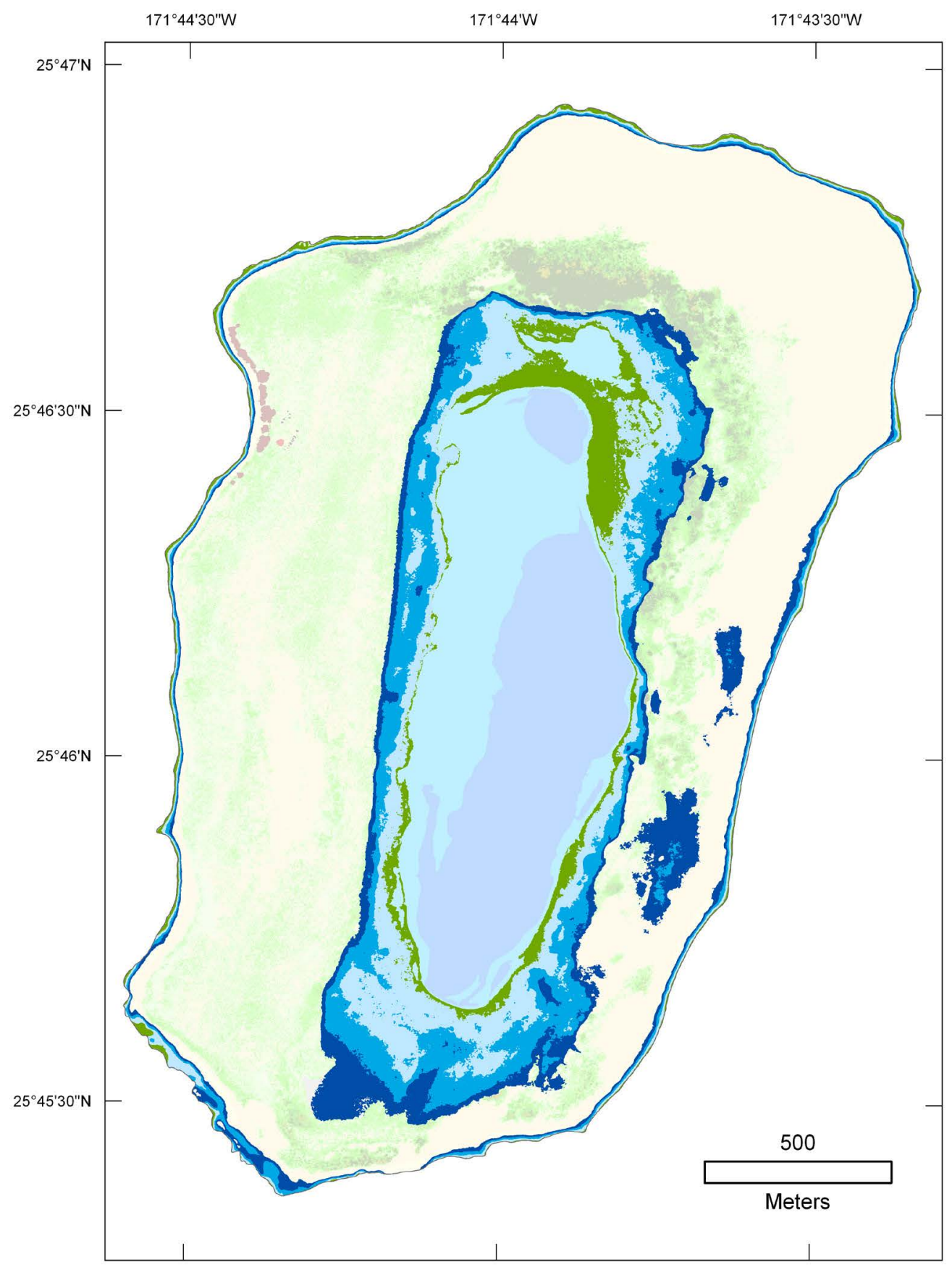

\begin{tabular}{|c|c|}
\hline \multirow{2}{*}{\multicolumn{2}{|c|}{$\begin{array}{l}\text { Overlay of Passive Inundation and Land Cover Class, } \\
\text { Assuming an Equal Rise in Groundwater and Lake Level } \\
\text { Inundation area by SLR scenario }\end{array}$}} \\
\hline & \\
\hline \multicolumn{2}{|r|}{$0.00 \mathrm{~m}$} \\
\hline \multicolumn{2}{|r|}{$+0.50 \mathrm{~m}$} \\
\hline \multicolumn{2}{|r|}{$+1.00 \mathrm{~m}$} \\
\hline \multicolumn{2}{|r|}{$+1.50 \mathrm{~m}$} \\
\hline \multicolumn{2}{|r|}{$+2.00 \mathrm{~m}$} \\
\hline \multicolumn{2}{|c|}{ Land cover class } \\
\hline & Tree/shrub \\
\hline ए & Casuarina equisetifolia \\
\hline$\square$ & Cocos nucifera \\
\hline ए & Pluchea indica \\
\hline Б & Tournefortia argentea \\
\hline$\square$ & Mixed shrub \\
\hline Б & Grass/herbaceous cover \\
\hline$\square$ & Vine/ground cover \\
\hline$\square$ & Wetland vegetation \\
\hline$\square$ & Bare ground \\
\hline Б & Hard pan \\
\hline$\square$ & Beach \\
\hline$\square$ & Wetland (unvegetated) \\
\hline$\square$ & Wetland (standing water) \\
\hline$\square$ & Human structures \\
\hline & Shoreline (mean sea level) \\
\hline
\end{tabular}

Figure 2.19. Overlay of passive inundation zones on Laysan Island for five sea-level rise (SLR) scenarios with land cover in the background (fig. 2.21 provides a clearer depiction of land cover). This map depicts passive SLR inundation along the ocean (as shown in fig. 2.12) plus potential inundation from rising groundwater and an expanded lake area. The pattern of potential groundwater inundation under this modeling approach resembles the inundation pattern exhibited by the wave-driven inundation model when wave-driven seawater volumes are unlimited. Both models forecast extensive inundation of the lowlying areas near the central lake. Abbreviations: $m$, meters. 


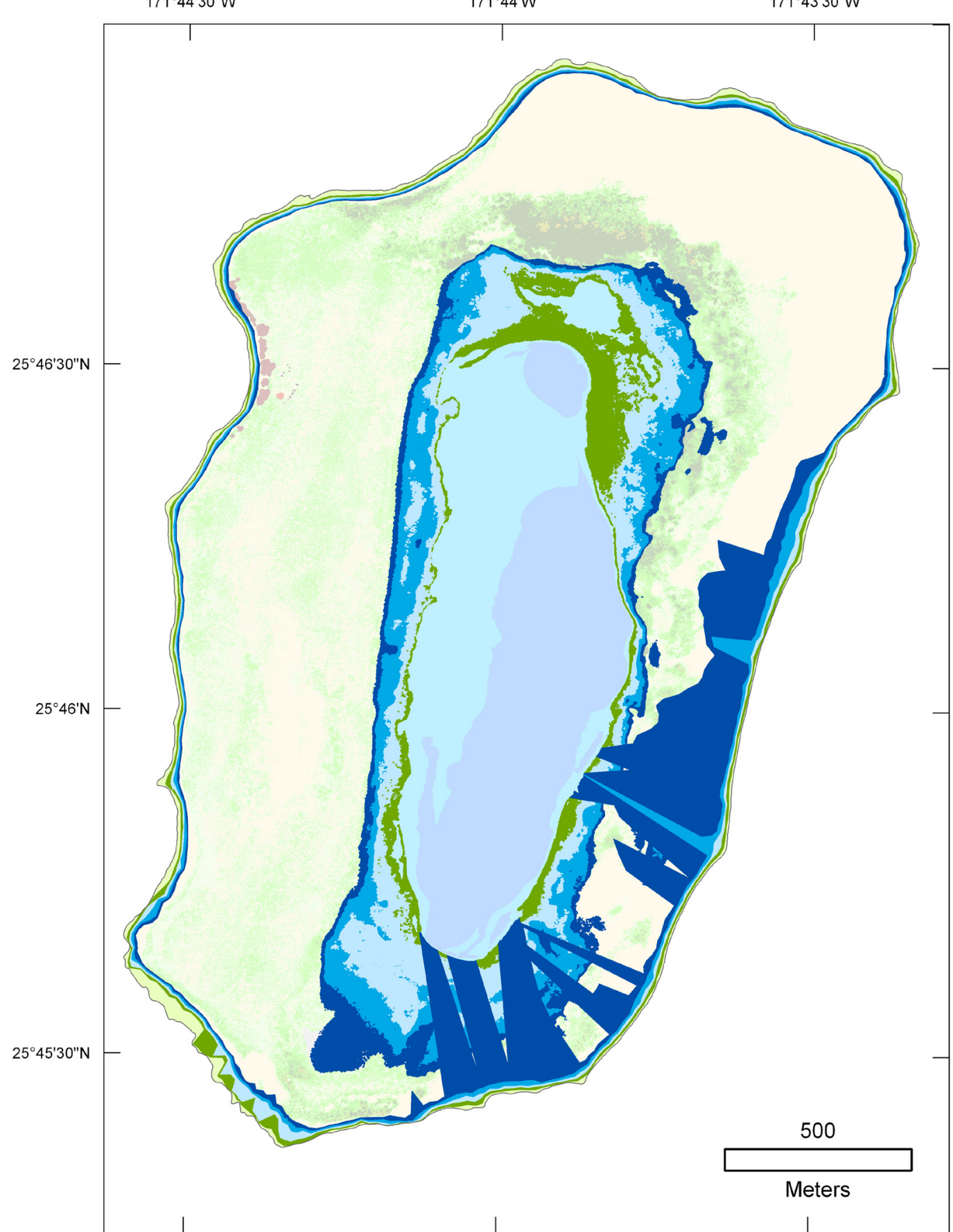

Overlay of Wave-Driven Inundation and Land Cover Class, Assuming an Equal Rise in Groundwater and Lake Level

Inundation area by SLR scenario

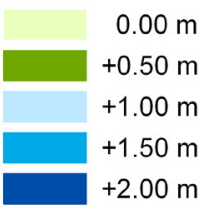

Land cover class

$\square$ Tree/shrub

Casuarina equisetifolia

Cocos nucifera

Pluchea indica

Tournefortia argentea

Mixed shrub

Grass/herbaceous cover

Vine/ground cover

Wetland vegetation

Bare ground

Hard pan

Beach

Wetland (unvegetated)

Wetland (standing water)

Human structures

Shoreline (mean sea level)

Figure 2.20. Overlay of wave-driven inundation including potential inundation from rising groundwater on Laysan Island for five sea-level rise (SLR) scenarios with land cover in the background (fig. 2.21 provides a clearer depiction of land cover). For the higher SLR scenarios (+1.50 and +2.00 meters, $\mathrm{m})$, the above inundation pattern covers substantially the same area as the inundation pattern from the wave-driven inundation model with unlimited volumes of seawater and no infiltration.

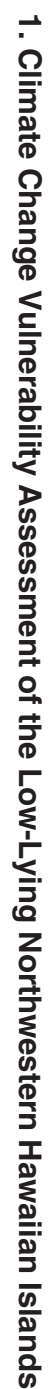


the wave-driven inundation model with unlimited seawater volumes and no infiltration. In terms of overall area, at +2.00 $\mathrm{m}$ SLR the passive inundation model (with rising groundwater) predicted a 27.4-percent reduction in terrestrial area.

Lastly, the model that incorporated wave-driven inundation and groundwater rise (fig. 2.20) produced an inundation pattern that closely resembled the inundation pattern of the wave-driven inundation model with unlimited seawater volumes and no infiltration. In terms of total area, this model predicted a 36.1-percent reduction in terrestrial area.

\section{Land Cover on Laysan Island}

We identified and quantified land cover for Laysan Island prior to considering the overlays of land cover and inundation (table 2.3, fig. 2.21). At the time the satellite imagery was captured, the lake basin (including seasonal mudflats and standing water) covered 18.0 percent (74.2 ha) of total island area. Of the remaining terrestrial land, 77.6 percent was composed of an interspersed mix of grass/herbaceous cover (22.1 percent), vine/ground cover (17.2 percent), and bare ground (38.3 percent). None of the other land cover classes, including tree/shrub, mixed shrub, wetland vegetation, and beach, individually covered greater than 6 percent of Laysan's terrestrial area. In general, land cover on Laysan featured a narrow band of beach around the island's perimeter, a narrow band of wetland vegetation around the lake perimeter, broad areas of sandy bare ground in the north and east, and predominantly interspersed bunch grass (Eragrostis variabilis) and beach morning glory (Ipomoea pes-caprae) elsewhere (especially in the west; fig. 2.21).

\section{Inundation by Land Cover Class}

With the passive inundation model, the only land cover class to exhibit substantial losses was beach (fig. 2.12, table $2.4 \mathrm{a})$. Even at the highest SLR scenario $(+2.00 \mathrm{~m})$, only 1.3 percent of the bare ground class was inundated under a passive modeling approach. Similar to the passive inundation model, the wave-driven inundation model (fig. 2.13, table 2.4b) exhibited losses predominantly to the beach area at SLR scenarios below $+2.00 \mathrm{~m}$. However, at $+2.00 \mathrm{~m} \mathrm{SLR}$, 96.6 percent of current beach area and nearly 20 percent of the bare ground class experienced periodic inundation during large-wave events. At the lower SLR scenarios (0.00, +0.50 , and $+1.00 \mathrm{~m}$ ), no differences existed between the wave-driven inundation models with and without unlimited seawater volumes. For the SLR scenarios of +1.50 and +2.00 $\mathrm{m}$, where unlimited seawater volumes flowed outward into adjacent depressions, broad flooding occurred across several land cover categories (fig. 2.18, table 2.4c). For most land cover classes, the extent of flooding did not differ greatly

Table 2.3. Area of each land cover class on Laysan Island, including percentage of total terrestrial area and percentage of total island area.

[The two unvegetated wetland classes are excluded from total terrestrial area. Abbreviations: ha, hectares; \%, percentage]

\begin{tabular}{lrcc}
\hline \multicolumn{1}{c}{ Land cover class } & Area (ha) & $\begin{array}{c}\text { \% of total } \\
\text { terrestrial } \\
\text { area }\end{array}$ & $\begin{array}{c}\text { \% of total } \\
\text { island area }\end{array}$ \\
\hline Tree/shrub & 12.3 & 3.6 & 3.0 \\
Casuarina equisetifolia & 0.0 & 0.0 & 0.0 \\
Cocos nucifera & 0.0 & 0.0 & 0.0 \\
Pluchea indica & 8.3 & 2.5 & 2.0 \\
Tournefortia argentea & 0.7 & 0.2 & 0.2 \\
Mixed shrub & 18.0 & 5.3 & 4.4 \\
Grass/herbaceous cover & 74.8 & 22.1 & 18.2 \\
Vine/ground cover & 58.0 & 17.2 & 14.1 \\
Wetland vegetation & 13.8 & 4.1 & 3.4 \\
Bare ground & 129.3 & 38.3 & 31.4 \\
Hard pan & 3.1 & 0.9 & 0.7 \\
Beach & 19.4 & 5.7 & 4.7 \\
Wetland (unvegetated) & 34.2 & --- & 8.3 \\
Wetland (standing water) & 40.0 & --- & 9.7 \\
Human structures & 0.0 & 0.0 & 0.0 \\
\hline Total terrestrial area & 337.8 & 100.0 & --- \\
Total island area & 412.0 & --- & 100.0
\end{tabular}




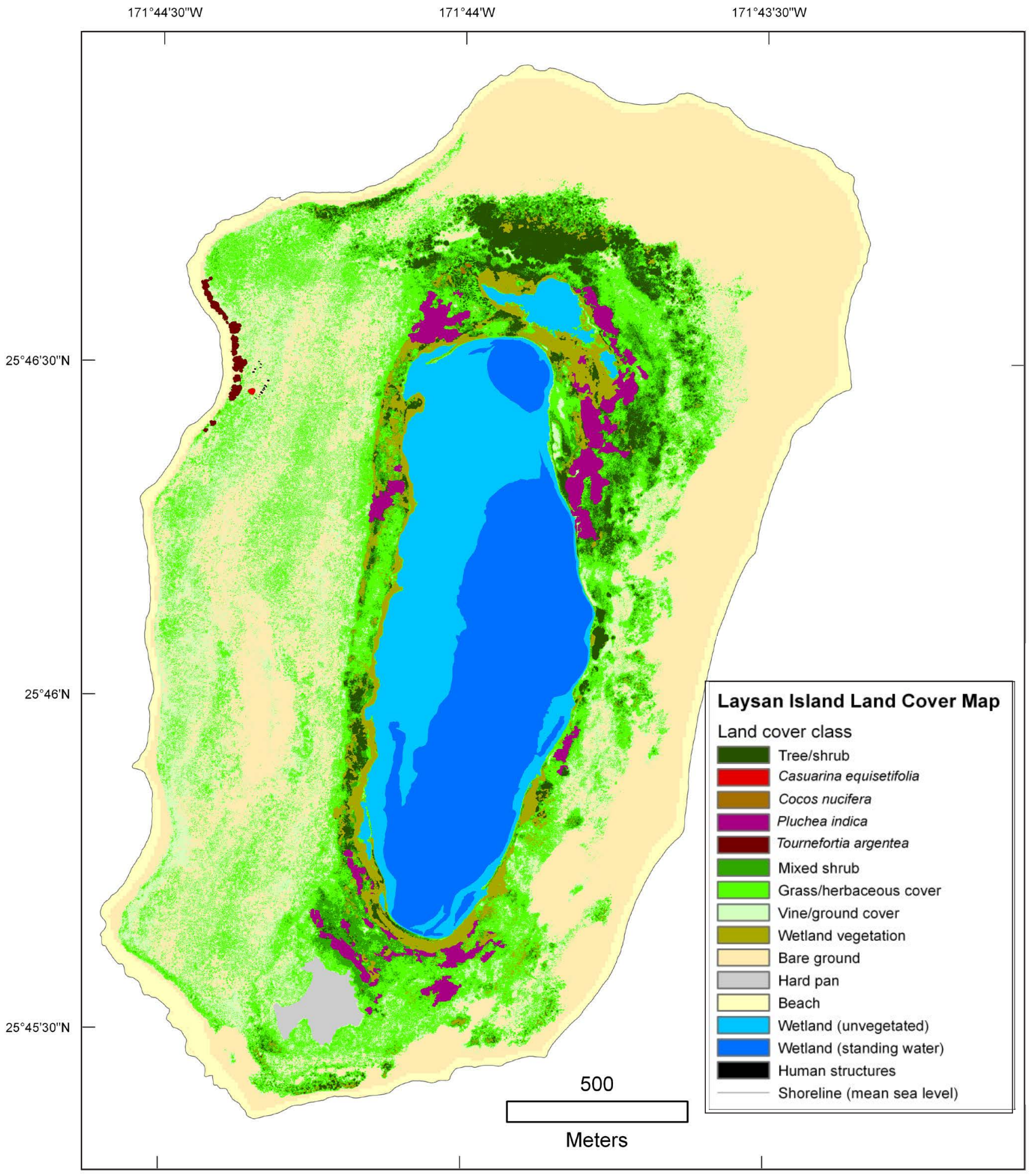

Figure 2.21. Land cover classes for Laysan Island, based on U.S. Fish and Wildlife Service ground-survey data (Hammond and others, 2010) and unsupervised classification of WorldView-2 satellite imagery taken on May 18, 2010 (Digital Globe Inc., 2010). This map provides the basis for determining the type of land cover and habitat that may be inundated under various sea-level rise (SLR) scenarios and modeling approaches. This map displays land cover class without using semi-transparent colors, as in previous maps. 
between the two highest scenarios $(+1.50$ and $+2.00 \mathrm{~m})$. Under both scenarios, losses were 100 percent for Cocos nucifera and Pluchea indica, and exceeded 60 percent for mixed shrub, 40 percent for grass/herbaceous cover, 90 percent for wetland vegetation, 95 percent for hard pan, and 85 percent for beach (table 2.4c). Managers with USFWS have been attempting to eradicate the invasive plant $P$. indica (Hawaiian Ecosystems at Risk Project, 2010) since 2010; thus any future losses to this land cover class may apply to the restored wetland or shrub species that replace $P$. indica.

The models that incorporated groundwater (tables $2.4 \mathrm{~d}$ and $2.4 \mathrm{e}$ ) exhibited similar losses in land cover types to the wavedriven inundation models with unlimited seawater volumes and no infiltration, as substantially the same low-lying areas would be inundated under either modeling approach. With the passive inundation model that includes rising groundwater, primarily wetland vegetation was inundated (38.6-percent loss) at +0.50 $\mathrm{m}$ SLR, whereas at $+1.00 \mathrm{~m} \mathrm{SLR}$, other classes including tree/shrub, C. nucifera, P. indica, and mixed shrub also were inundated (table 2.4d, fig. 2.19). The federally endangered plant Cyperus pennatiformis var. bryanii would be one of the species likely to experience substantial inundation under a scenario of rising groundwater (See chap. 1, Laysan Island Results, for additional details and $C$. pennatiformis var. bryanii distribution maps). At the higher SLR scenarios $(+1.50$ and $+2.00 \mathrm{~m})$, sizable losses to most land cover classes occurred. Lastly, for the wave-driven inundation model that incorporates rising groundwater (table 2.4e, fig. 2.20), specific losses by land cover class generally approached the magnitude of losses exhibited by the wave-driven inundation model with unlimited seawater volumes and no infiltration.

\section{Impacts of SLR on Nesting Habitat}

Vulnerability of nesting habitat to SLR and wave-driven inundation varied according to species distribution and breeding ecology (figs. 2.22-28), as well as by the SLR scenario and modeling approach: (1) passive inundation model, (2) wavedriven inundation model, (3) wave-driven inundation model assuming unlimited seawater volumes and no infiltration, (4) passive inundation model with rising groundwater, and (5) wave-driven inundation model with rising groundwater.

Table 2.4. Inundated areas and percent change for Laysan Island, tallied by land cover class for five sea-level rise (SLR) scenarios $(0.00,+0.50,+1.00,+1.50$, and +2.00 meters) under five modeling approaches: (a) passive inundation, (b) dynamic wave-driven inundation, (c) dynamic wave-driven inundation with unlimited seawater volumes and no infiltration, (d) passive inundation including rising groundwater levels, and (e) dynamic wave-driven inundation including rising groundwater levels.

[All values reflect inundation at mean high water (MHW) for the specified SLR scenario. For example, at present sea level (SLR $=0.00 \mathrm{~m})$, inundation represents the area above mean sea level that is submerged at MHW. Abbreviations: ha, hectares; \%, percent; $\mathrm{m}$, meters]

a. Passive Inundation Model

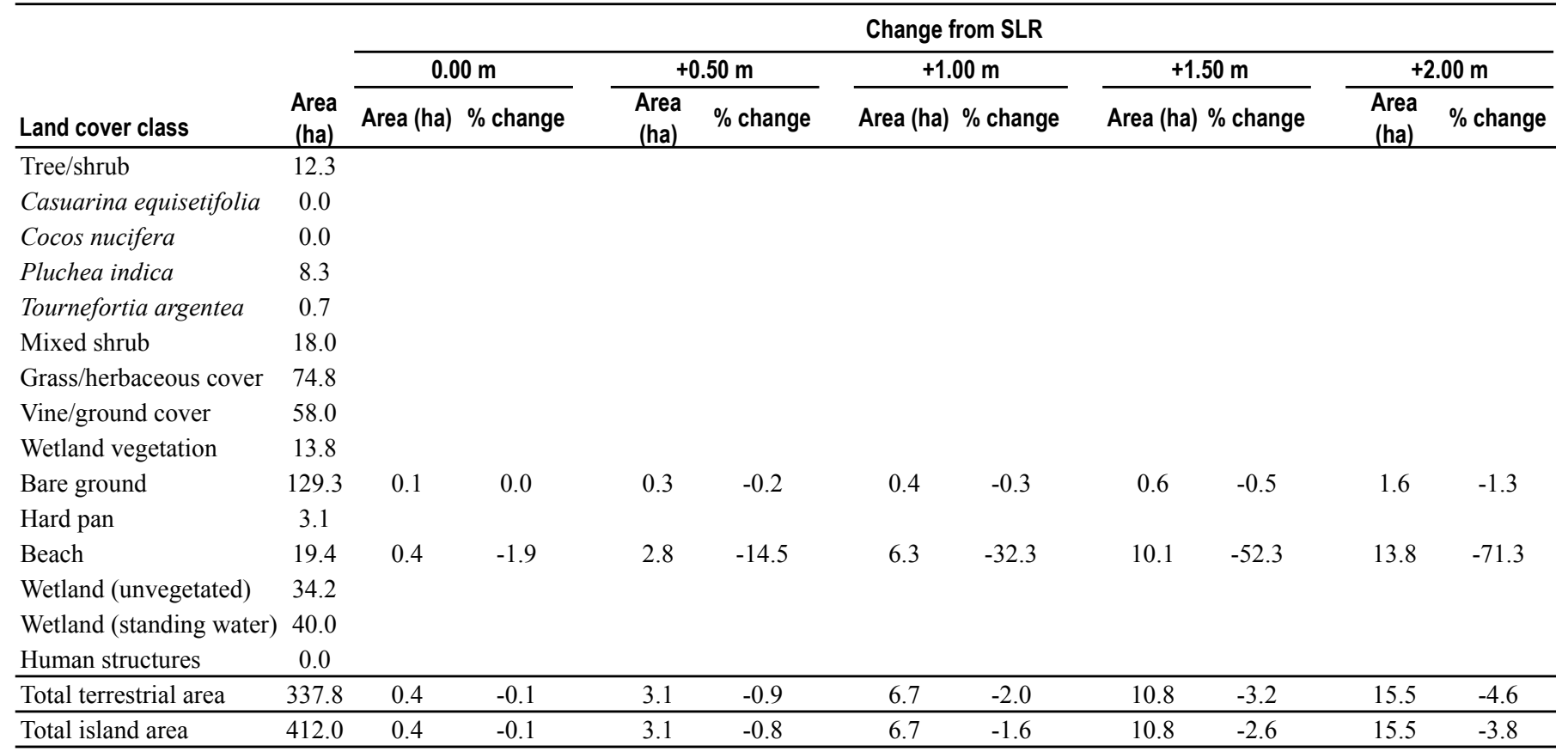


Table 2.4. Inundated areas and percent change for Laysan Island, tallied by land cover class for five sea-level rise (SLR) scenarios $(0.00,+0.50,+1.00,+1.50$, and +2.00 meters $)$ under five modeling approaches: (a) passive inundation, (b) dynamic wave-driven inundation, (c) dynamic wave-driven inundation with unlimited seawater volumes and no infiltration, (d) passive inundation including rising groundwater levels, and (e) dynamic wave-driven inundation including rising groundwater levels. - Continued

[All values reflect inundation at mean high water (MHW) for the specified SLR scenario. For example, at present sea level (SLR $=0.00 \mathrm{~m})$, inundation represents the area above mean sea level that is submerged at MHW. Abbreviations: ha, hectares; \%, percent; $m$, meters]

b. Dynamic Wave-driven Inundation Model

\begin{tabular}{|c|c|c|c|c|c|c|c|c|c|c|c|}
\hline \multirow[b]{3}{*}{ Land cover class } & \multirow[b]{3}{*}{ Area (ha) } & \multicolumn{10}{|c|}{ Change from SLR } \\
\hline & & \multicolumn{2}{|c|}{$0.00 \mathrm{~m}$} & \multicolumn{2}{|c|}{$+0.50 \mathrm{~m}$} & & \multicolumn{2}{|c|}{$+1.50 \mathrm{~m}$} & \multicolumn{2}{|c|}{$+2.00 \mathrm{~m}$} \\
\hline & & Area (ha) & $\%$ change & $\begin{array}{l}\text { Area } \\
\text { (ha) }\end{array}$ & $\%$ change & Area (ha) & $\%$ change & Area (ha) & $\%$ change & $\begin{array}{l}\text { Area } \\
\text { (ha) }\end{array}$ & $\%$ change \\
\hline Tree/shrub & 12.3 & & & & & & & 0.0 & -0.1 & 0.1 & -1.0 \\
\hline Casuarina equisetifolia & 0.0 & & & & & & & & & & \\
\hline Cocos nucifera & 0.0 & & & & & & & & & & \\
\hline Mixed shrub & 18.0 & & & & & & & & & 1.0 & -5.6 \\
\hline Grass/herbaceous cover & 74.8 & & & & & & & 0.1 & -0.1 & 6.9 & -9.3 \\
\hline Vine/ground cover & 58.0 & & & & & & & 0.1 & -0.1 & 3.7 & -6.3 \\
\hline Wetland vegetation & 13.8 & & & & & & & & & 1.2 & -8.4 \\
\hline Bare ground & 129.3 & 0.4 & -0.3 & 0.6 & -0.5 & 1.5 & -1.1 & 4.9 & -3.8 & 25.2 & -19.5 \\
\hline Human structures & 0.0 & & & & & & & & & & \\
\hline Total terrestrial area & 337.8 & 6.1 & -1.8 & 10.5 & -3.1 & 15.5 & -4.6 & 22.1 & -6.5 & 57.9 & -17.2 \\
\hline Total island area & 412.0 & 6.1 & -1.5 & 10.5 & -2.5 & 15.5 & -3.8 & 22.1 & -5.4 & 57.9 & -14.1 \\
\hline
\end{tabular}

c. Dynamic Wave-driven Inundation Model with Unlimited Seawater Volumes and No Infiltration

\begin{tabular}{|c|c|c|c|c|c|c|c|c|c|c|c|}
\hline \multirow[b]{3}{*}{ Land cover class } & \multirow[b]{3}{*}{ Area (ha) } & \multicolumn{10}{|c|}{ Change from SLR } \\
\hline & & \multicolumn{2}{|c|}{$0.00 \mathrm{~m}$} & \multicolumn{2}{|c|}{$+0.50 \mathrm{~m}$} & \multicolumn{2}{|c|}{$+1.00 \mathrm{~m}$} & \multicolumn{2}{|c|}{$+1.50 \mathrm{~m}$} & \multicolumn{2}{|c|}{$+2.00 \mathrm{~m}$} \\
\hline & & Area (ha) & $\%$ change & $\begin{array}{l}\text { Area } \\
\text { (ha) }\end{array}$ & $\%$ change & Area (ha) & $\%$ change & Area (ha) & $\%$ change & $\begin{array}{l}\text { Area } \\
\text { (ha) }\end{array}$ & $\%$ change \\
\hline Tree/shrub & 12.3 & & & & & & & 6.2 & -50.3 & 6.4 & -52.1 \\
\hline Casuarina equisetifolia & 0.0 & & & & & & & & & & \\
\hline Cocos nucifera & 0.0 & & & & & & & 0.0 & -100.0 & 0.0 & -100.0 \\
\hline Mixed shrub & 18.0 & & & & & & & 11.7 & -64.9 & 12.2 & -68.0 \\
\hline Grass/herbaceous cover & 74.8 & & & & & & & 31.1 & -41.6 & 33.9 & -45.3 \\
\hline Vine/ground cover & 58.0 & & & & & & & 8.7 & -15.0 & 10.8 & -18.5 \\
\hline Wetland vegetation & 13.8 & & & & & & & 12.5 & -90.8 & 12.7 & -91.6 \\
\hline Bare ground & 129.3 & 0.4 & -0.3 & 0.6 & -0.5 & 1.5 & -1.1 & 17.2 & -13.3 & 30.1 & -23.2 \\
\hline Human structures & 0.0 & & & & & & & & & & \\
\hline Total terrestrial area & 337.8 & 6.1 & -1.8 & 10.5 & -3.1 & 15.5 & -4.6 & 115.8 & -34.3 & 136.2 & -40.3 \\
\hline Total island area & 412.0 & 6.1 & -1.5 & 10.5 & -2.5 & 15.5 & -3.8 & 115.8 & -28.1 & 136.2 & -33.1 \\
\hline
\end{tabular}


Table 2.4. Inundated areas and percent change for Laysan Island, tallied by land cover class for five sea-level rise (SLR) scenarios $(0.00,+0.50,+1.00,+1.50$, and +2.00 meters $)$ under five modeling approaches: (a) passive inundation, (b) dynamic wave-driven inundation, (c) dynamic wave-driven inundation with unlimited seawater volumes and no infiltration, (d) passive inundation including rising groundwater levels, and (e) dynamic wave-driven inundation including rising groundwater levels. - Continued

[All values reflect inundation at mean high water (MHW) for the specified SLR scenario. For example, at present sea level (SLR $=0.00 \mathrm{~m})$, inundation represents the area above mean sea level that is submerged at MHW. Abbreviations: ha, hectares; \%, percent; $m$, meters]

d. Passive Inundation Model Including Rising Groundwater Levels

\begin{tabular}{|c|c|c|c|c|c|c|c|c|c|c|c|}
\hline \multirow[b]{3}{*}{ Land cover class } & \multirow[b]{3}{*}{ Area (ha) } & \multicolumn{10}{|c|}{ Change from SLR } \\
\hline & & \multicolumn{2}{|c|}{$0.00 \mathrm{~m}$} & \multicolumn{2}{|c|}{$+0.50 \mathrm{~m}$} & \multicolumn{2}{|c|}{$+1.00 \mathrm{~m}$} & \multicolumn{2}{|c|}{$+1.50 \mathrm{~m}$} & \multicolumn{2}{|c|}{$+2.00 \mathrm{~m}$} \\
\hline & & Area (ha) & $\%$ change & $\begin{array}{l}\text { Area } \\
\text { (ha) }\end{array}$ & $\%$ change & Area (ha) & $\%$ change & Area (ha) & $\%$ change & $\begin{array}{c}\text { Area } \\
\text { (ha) }\end{array}$ & $\%$ change \\
\hline Casuarina equisetifolia & 0.0 & & & & & & & & & & \\
\hline Cocos nucifera & 0.0 & & & 0.0 & -1.9 & 0.0 & -100.0 & 0.0 & -100.0 & 0.0 & -100.0 \\
\hline Pluchea indica & 8.3 & & & 0.3 & -4.0 & 6.5 & -78.5 & 8.3 & -100.0 & 8.3 & -100.0 \\
\hline Mixed shrub & 18.0 & & & 1.1 & -5.9 & 5.8 & -32.5 & 9.9 & -55.2 & 10.9 & -60.4 \\
\hline Grass/herbaceous cover & 74.8 & & & 2.7 & -3.6 & 7.6 & -10.1 & 19.6 & -26.2 & 26.2 & -35.0 \\
\hline Vine/ground cover & 58.0 & & & 0.4 & -0.6 & 0.7 & -1.3 & 2.8 & -4.9 & 5.5 & -9.6 \\
\hline Wetland vegetation & 13.8 & & & 5.3 & -38.6 & 9.9 & -71.4 & 12.2 & -88.1 & 12.4 & -89.9 \\
\hline Bare ground & 129.3 & 0.1 & 0.0 & 0.3 & -0.2 & 0.4 & -0.3 & 1.3 & -1.0 & 6.9 & -5.4 \\
\hline Human structures & 0.0 & & & & & & & & & & \\
\hline Total terrestrial area & 337.8 & 0.4 & -0.1 & 13.8 & -4.1 & 40.8 & -12.1 & 70.2 & -20.8 & 92.6 & -27.4 \\
\hline Total island area & 412.0 & 0.4 & -0.1 & 13.8 & -3.4 & 40.8 & -9.9 & 70.2 & -17.0 & 92.6 & -22.5 \\
\hline
\end{tabular}

e. Dynamic Wave-driven Inundation Model Including Rising Groundwater Levels

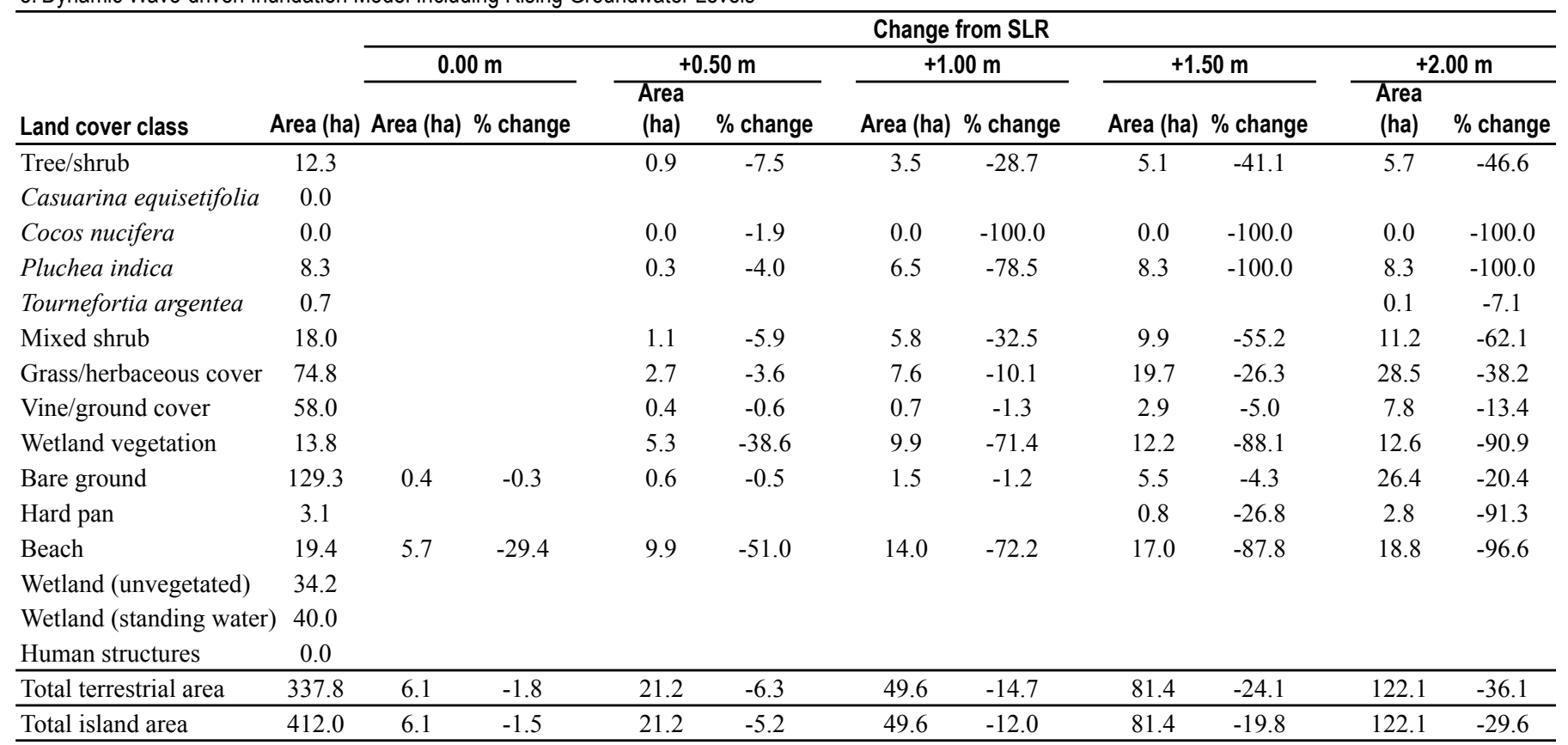


Based on the spatial distribution of 2010-11 breeding season data, Black-footed Albatross (BFAL) nested on 191.2 ha located primarily around the island's perimeter (fig. 2.22). BFAL nested in all habitats, excluding areas of continuous tree/shrub cover, wetland vegetation, and the narrow strip of beach (subject to tidal fluctuations and typical wave swash). Under the passive modeling approach, less than 1 percent of BFAL nesting area experienced inundation at any SLR scenario considered (table 2.5). When groundwater rise was incorporated into the passive model, 6.1 percent of BFAL nesting area was inundated at the highest SLR scenario $(+2.00 \mathrm{~m})$; for all other SLR scenarios, less than 2 percent of their nesting area experienced inundation, as BFAL are not typically found nesting in the low-lying central basin. Using the wave-driven modeling approach with $+2.00 \mathrm{~m}$ of SLR, inundation covered 16.3 percent (limited seawater-volume case) or 21.5 percent (unlimited seawater-volume case) of the BFAL nesting area. At $+1.50 \mathrm{~m} \mathrm{SLR}$, inundation covered 13.4 percent of the BFAL nesting area for the unlimited seawater scenario (table 2.5).

Current spatial distribution data based on surveys were not available for the Laysan Albatross (LAAL); however, using our land cover classification, we estimated their potential nesting habitat as 297.0 ha (fig. 2.23). Passive inundation models for all SLR scenarios (including the +2.00 -m case)

Table 2.5. Inundation of 2009-2010 nesting habitat at Laysan Island (USFWS data; Kristof and others, 2010) for Blackfooted Albatross and Laysan Albatross under five sea-level rise (SLR) scenarios and five modeling approaches: (1) passive inundation, (2) dynamic wave-driven inundation, (3) dynamic wave-driven inundation with unlimited seawater volumes and no infiltration, (4) passive SLR including a comparable rise in groundwater, and (5) dynamic wave-driven inundation including a comparable rise in groundwater.

[Inundation of nesting habitat is expressed in area and percent change relative to the total habitat without inundation (listed in last row). Abbreviations: USFWS, U.S. Fish and Wildlife Service; m, meters; ha, hectares; \%, percent]

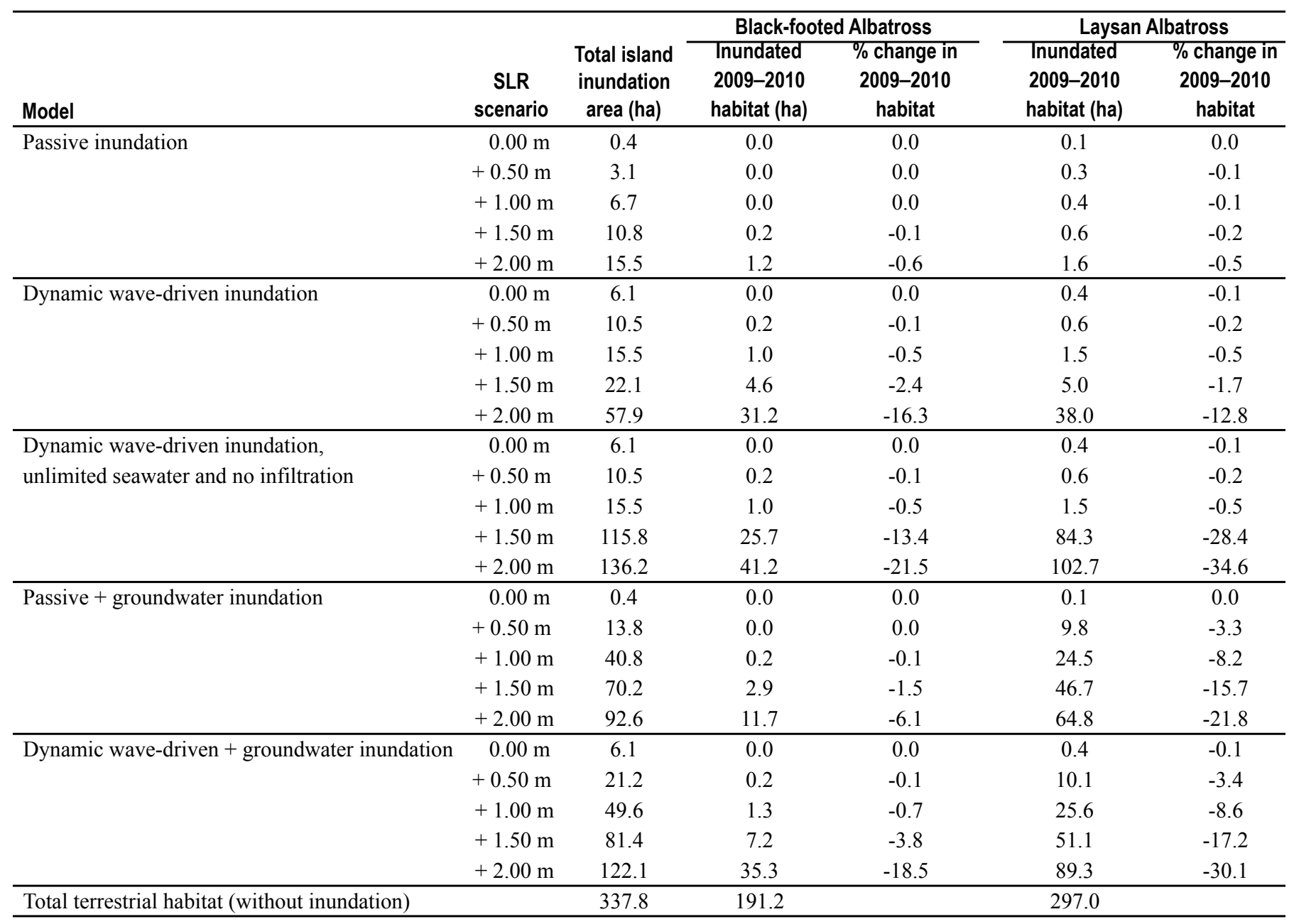


$171^{\circ} 44^{\prime} \mathrm{W}$

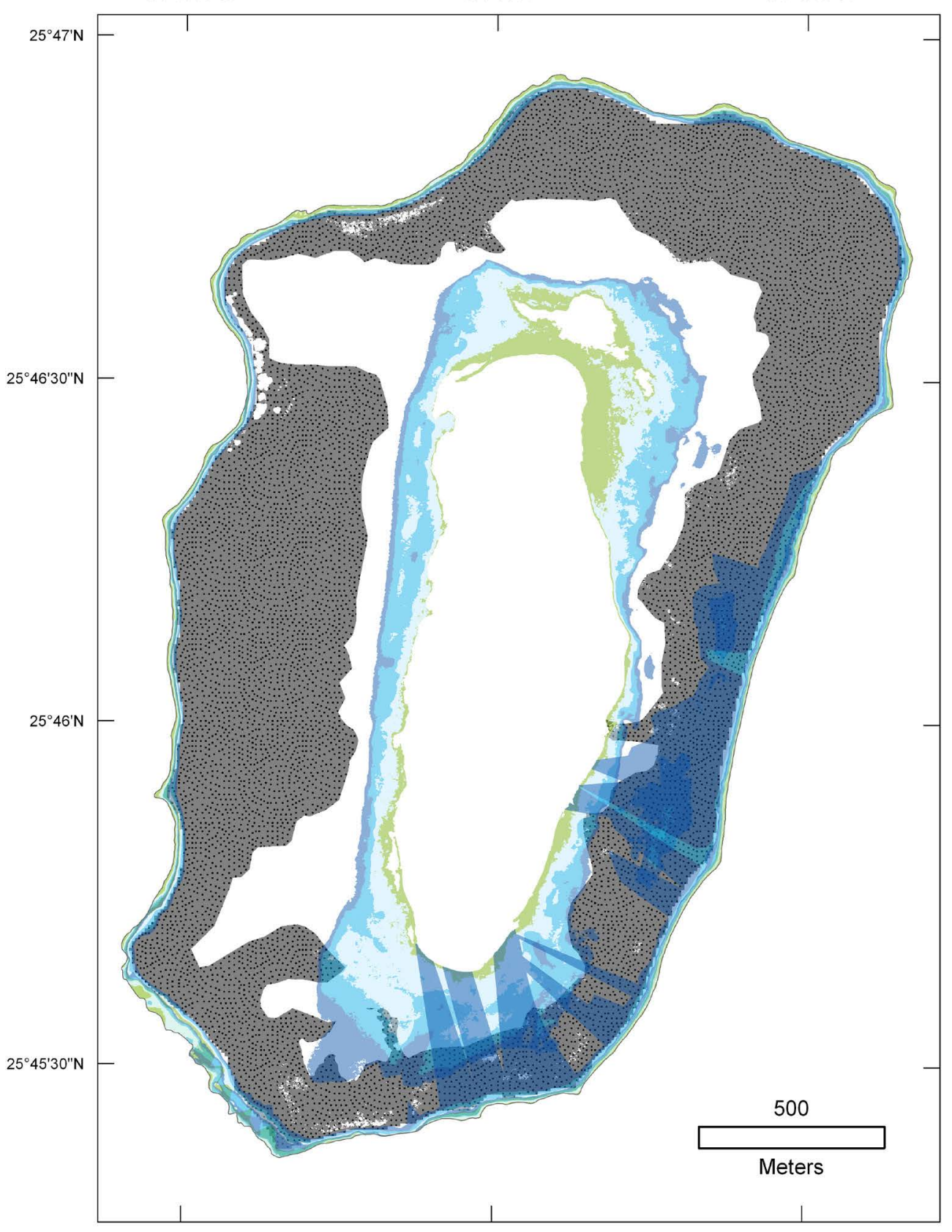

Overlay of 2009-2010 Black-Footed Albatross Nesting Habitat and Combined Inundation Patterns Black-footed Albatross nesting habitat

Inundation area by SLR scenario
$0.00 \mathrm{~m}$
$+0.50 \mathrm{~m}$
$+1.00 \mathrm{~m}$
$+1.50 \mathrm{~m}$
$+2.00 \mathrm{~m}$
Shoreline (mean sea level)

Figure 2.22. Overlay of Black-footed Albatross (BFAL) nesting habitat at Laysan Island, with combined inundation from passive sea-level rise (SLR), wave-driven water levels, and rising groundwater. The combined background allows for a comparison of inundation extents from various models with BFAL nesting habitat. By depicting inundation extent with semi-transparent symbols, the underlying nesting habitat remains visible, although may appear slightly darker. Biologists performed a census of BFAL nest sites in December 2009 and January 2010 U.S. Fish and Wildlife Service (USFWS) data; Kristof and others, 2010); they also conducted direct counts. BFAL nested on 191.2 hectares (ha) located primarily around the island's perimeter during the 2010-11 breeding season. Under the passive modeling approach, less than 1 percent of BFAL nesting area experienced inundation for all SLR scenarios. When groundwater rise was incorporated into the passive model, 6.1 percent of BFAL nesting area was inundated at the highest SLR scenario $(+2.00$ meters, $\mathrm{m})$. With the wave-driven modeling approach at $+2.00 \mathrm{~m} \mathrm{SLR}$, inundation covered 16.3 percent (limited seawater-volume case) or 21.5 percent (unlimited seawater-volume case) of the BFAL nesting area. 


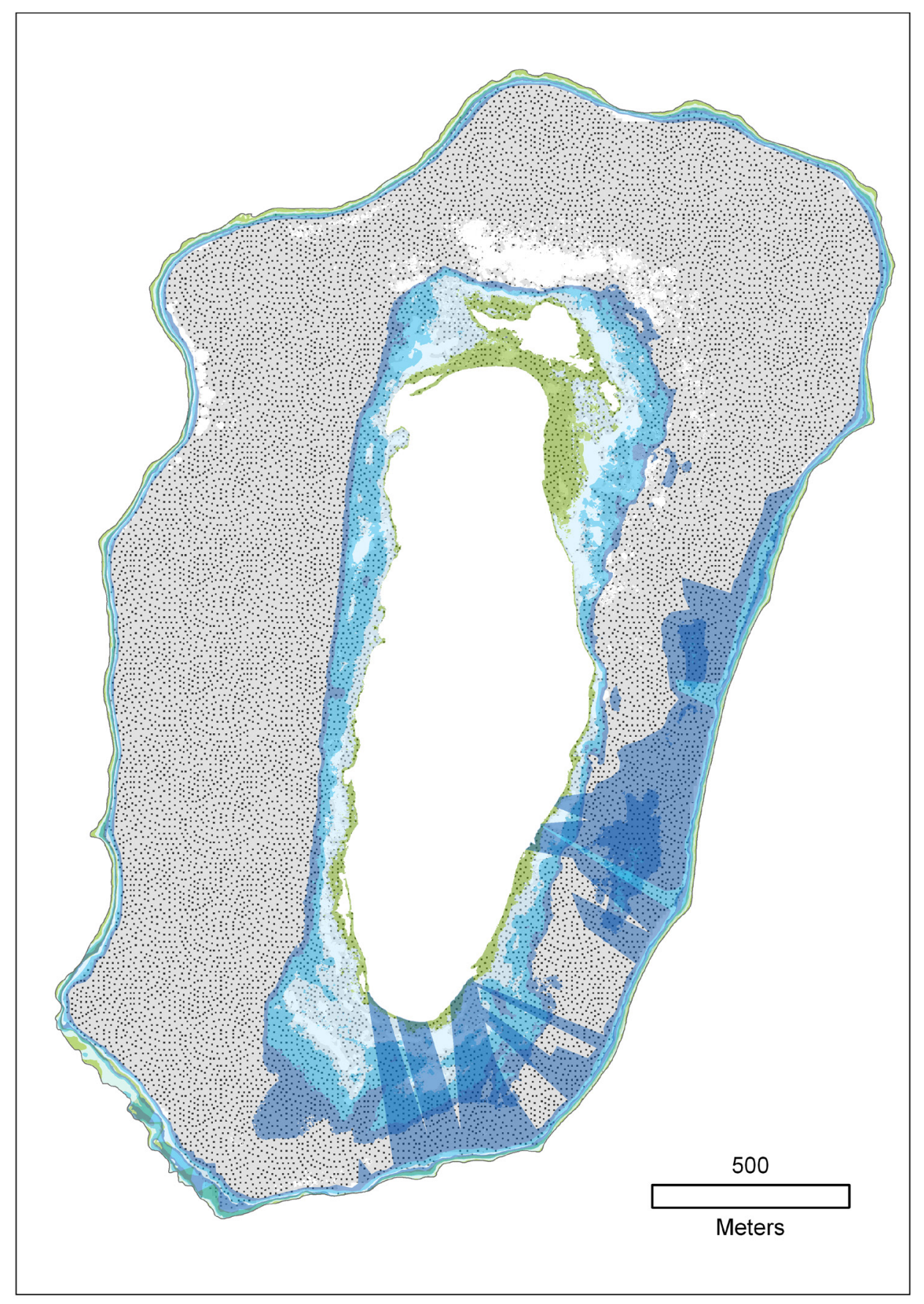

\begin{tabular}{l} 
Overlay of Laysan Albatross Nesting Habitat \\
and Combined Inundation Patterns \\
Inundation area by SLR scenario \\
$0.00 \mathrm{~m}$ \\
$+0.50 \mathrm{~m}$ \\
$+1.00 \mathrm{~m}$ \\
$+1.50 \mathrm{~m}$ \\
$+2.00 \mathrm{~m}$ \\
Shoreline (mean sea level) \\
\hline
\end{tabular}

Figure 2.23. Overlay of Laysan Albatross (LAAL) nesting habitat at Laysan Island, with combined inundation from passive sealevel rise (SLR), wave-driven water levels, and rising groundwater. The combined background allows for a comparison of inundation extents from various models with LAAL nesting habitat. By depicting inundation extent with semi-transparent symbols, the underlying nesting habitat remains visible, although may appear slightly darker. U.S. Fish and Wildlife Service (USFWS) biologists surveyed LAAL nests in 2010 (USFWS data; Kristof and others, 2010). The displayed nesting habitat was based on land cover maps and known nesting habitats. We estimated the current potential nesting habitat for LAAL as 297.0 hectares (ha). Passive inundation models for all SLR scenarios predicted LAAL habitat loss of less than 1 percent. At +2.00 meters $(m)$ SLR, the other modeling approaches (including the wave-driven and rising groundwater models) exhibited moderate to substantial levels of LAAL habitat inundation (12.8 to 34.6 percent). Likewise, when we considered either unlimited volumes of seawater or rising groundwater, the $+1.50 \mathrm{~m}$ scenario showed reasonably large habitat losses (15.7 to 28.4 percent). 
predicted LAAL habitat inundation of less than 1 percent (table 2.5). At $+2.00 \mathrm{~m}$ SLR, the other modeling approaches (including the wave-driven and groundwater approaches) exhibited moderate to substantial levels of LAAL habitat inundation (12.8 to 34.6 percent). Likewise, when we considered either unlimited volumes of seawater or rising groundwater, the +1.50 -m scenario resulted in large habitat losses (15.7 to 28.4 percent; table 2.5 ).

The spatial distribution of LAAL nests overlapped the area vulnerable to inundation from winter storms, wave-driven water levels, and rising groundwater (USFWS data; Kristof and others, 2011). The inundation risks to potential habitat (that is, potential habitat based on land cover classes derived from satellite imagery rather than ground survey data) for BFAL and LAAL are similar. In general, potential habitat loss exceeded currently known BFAL nesting area loss (from ground survey data), as BFAL typically do not nest in the low-lying interior areas identified as habitat with land cover classification.

Masked Booby (MABO) nests $(\mathrm{n}=163)$ were distributed throughout much of the island's interior in 2009 (fig. 2.24). No MABO nests were inundated for any of the passive SLR scenarios up to $+2.00 \mathrm{~m}$ SLR (table 2.6). For the wave-driven inundation model at the highest SLR scenario $(+2.00 \mathrm{~m})$, 11.7 percent of the MABO nest sites experienced flooding. Incorporating rising groundwater into the passive model led to

Table 2.6. Inundation of 2009 nest sites at Laysan Island (USFWS data; Boyd and others, 2009) for Masked Booby $(n=163)$ and Brown Booby $(n=35)$ under five sea-level rise (SLR) scenarios and five modeling approaches: (1) passive inundation, (2) dynamic wave-driven inundation, (3) dynamic wave-driven inundation with unlimited seawater volumes and no infiltration, (4) passive SLR including a comparable rise in groundwater, and (5) dynamic wave-driven inundation including a comparable rise in groundwater.

[U.S. Fish and Wildlife Service biologists mapped all nests June 17-19, 2009. [Inundation of nest sites is expressed as a percent change relative to the 2009 census numbers. Abbreviations: USFWS, U.S. Fish and Wildlife Service; m, meters; ha, hectares; \%, percent]

\begin{tabular}{|c|c|c|c|c|}
\hline \multirow[b]{2}{*}{ Model } & \multirow[b]{2}{*}{$\begin{array}{c}\text { SLR } \\
\text { scenario }\end{array}$} & \multirow[b]{2}{*}{$\begin{array}{c}\text { Total } \\
\text { inundation } \\
\text { area (ha) }\end{array}$} & \multirow{2}{*}{$\begin{array}{c}\text { Masked Booby } \\
\% \text { change } \\
\text { from } 2009 \\
\text { nest sites }\end{array}$} & \multirow{2}{*}{$\begin{array}{c}\text { Brown Booby } \\
\% \text { change } \\
\text { from } 2009 \\
\text { nest sites }\end{array}$} \\
\hline & & & & \\
\hline \multirow[t]{4}{*}{ Passive inundation } & $0.00 \mathrm{~m}$ & 0.4 & 0.0 & 0.0 \\
\hline & $+0.50 \mathrm{~m}$ & 3.1 & 0.0 & 0.0 \\
\hline & $+1.00 \mathrm{~m}$ & 6.7 & 0.0 & 0.0 \\
\hline & $+2.00 \mathrm{~m}$ & 15.5 & 0.0 & 0.0 \\
\hline \multirow[t]{4}{*}{ Dynamic wave-driven inundation } & $0.00 \mathrm{~m}$ & 6.1 & 0.0 & 0.0 \\
\hline & $+0.50 \mathrm{~m}$ & 10.5 & 0.0 & 0.0 \\
\hline & $+1.00 \mathrm{~m}$ & 15.5 & 0.0 & 0.0 \\
\hline & $+1.50 \mathrm{~m}$ & 22.1 & 0.0 & 0.0 \\
\hline \multirow{3}{*}{ unlimited seawater and no infiltration } & $+1.00 \mathrm{~m}$ & 15.5 & 0.0 & 0.0 \\
\hline & $+1.50 \mathrm{~m}$ & 115.8 & -39.3 & -17.1 \\
\hline & $+2.00 \mathrm{~m}$ & 136.2 & -46.6 & -22.9 \\
\hline \multirow[t]{5}{*}{ Passive + groundwater inundation } & $0.00 \mathrm{~m}$ & 0.4 & 0.0 & 0.0 \\
\hline & $+0.50 \mathrm{~m}$ & 13.8 & -2.5 & 0.0 \\
\hline & $+1.00 \mathrm{~m}$ & 40.8 & -9.2 & -11.4 \\
\hline & $+1.50 \mathrm{~m}$ & 70.2 & -18.4 & -11.4 \\
\hline & $+2.00 \mathrm{~m}$ & 92.6 & -27.0 & -14.3 \\
\hline \multirow[t]{2}{*}{ Dynamic wave-driven + groundwater inundation } & $0.00 \mathrm{~m}$ & 6.1 & 0.0 & 0.0 \\
\hline & $+0.50 \mathrm{~m}$ & 21.2 & -2.5 & 0.0 \\
\hline
\end{tabular}




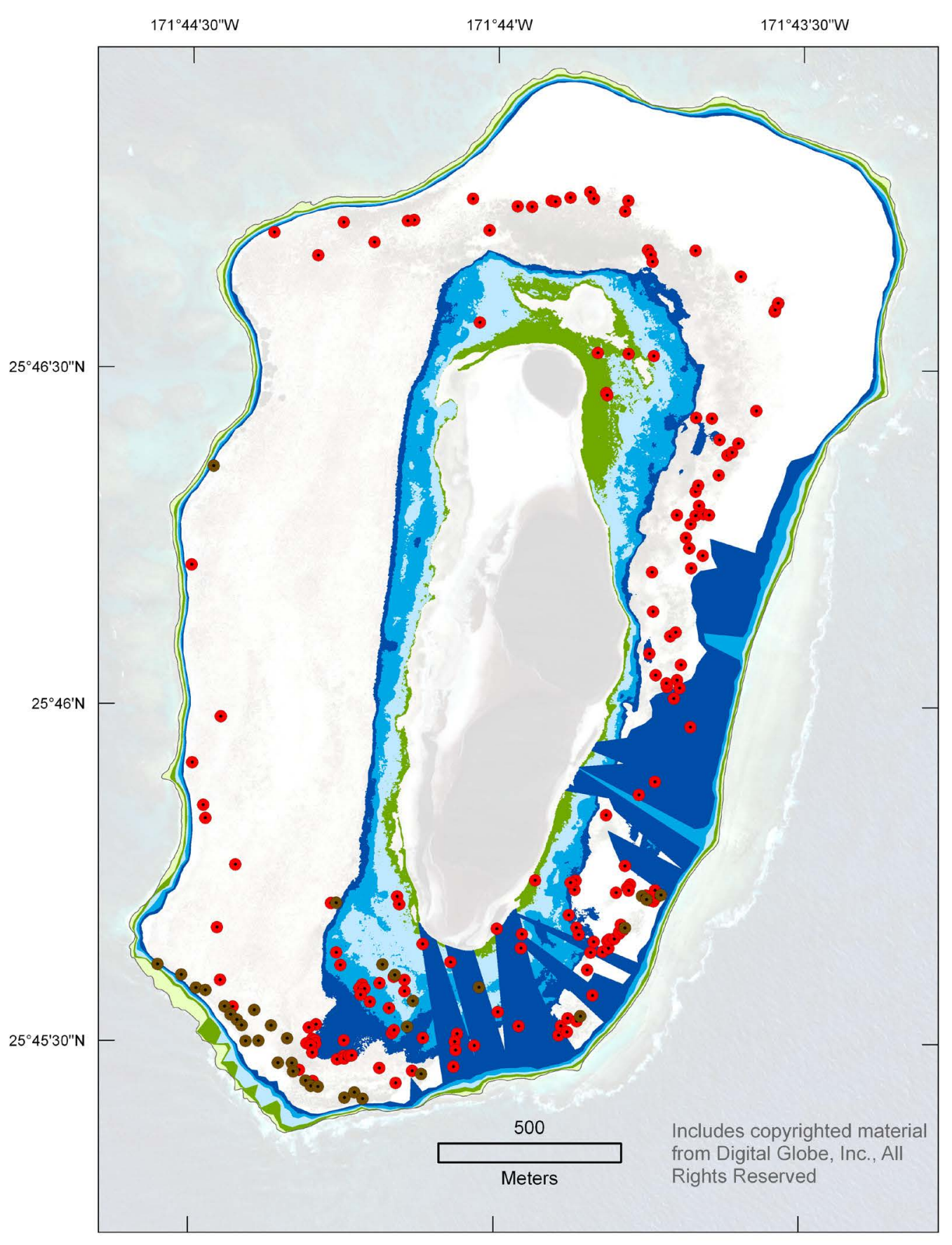

Overlay of 2009 Masked and Brown Booby Nest Sites with Combined Inundation Patterns

2009 nest sites

- Masked Booby

- Brown Booby

Inundation area by SLR scenario

$0.00 \mathrm{~m}$
$+0.50 \mathrm{~m}$
$+1.00 \mathrm{~m}$
$+1.50 \mathrm{~m}$
$+2.00 \mathrm{~m}$
+ Shoreline (mean sea level)

Figure 2.24. Overlay of Masked Booby (MABO, $n=163$ ) and Brown Booby (BRBO, $n=35$ ) nests mapped on Laysan Island in 2009, with combined inundation from passive sea-level rise (SLR), wave-driven water levels, and rising groundwater. The combined background allows for a comparison of inundation extents from various models with displayed nest sites. A semitransparent satellite image appears in the background (Digital Globe Inc., 2010). U.S. Fish and Wildlife Service (USFWS) biologists conducted a complete census of these two species June 17-19, 2009 (USFWS data; Boyd and others, 2009). MABO nested across the island interior especially in the north, east and south, whereas BRBO nested primarily along the southwestern edge of the island. Neither species located their nests in the coastal inundation zone for any of the passive SLR scenarios considered; however, when an equal rise in groundwater was modeled, 27.0 percent of MABO nests and 14.3 percent of BRBO nests experienced inundation at +2.00 meters $(m) S L R$. For the wave-driven inundation models with limited seawater volumes, no MABO or BRBO nests were inundated until SLR reached $+2.00 \mathrm{~m}$. 
greater inundation rates, with 18.4 percent and 27.0 percent of the nest sites flooded at +1.50 and $+2.00 \mathrm{~m}$ SLR, respectively. Greater rates of inundation occurred with the wave-driven modeling approach assuming unlimited seawater volumes and no infiltration, with as much as 46.6 percent of the nest sites experiencing flooding at a SLR scenario of $+2.00 \mathrm{~m}$.

Nest sites ( $n=35)$ of Brown Booby (BRBO) were concentrated inland of the protective dunes on the southwest coast in 2009; consequently, no inundation of nest sites occurred under the passive model (fig. 2.24). When groundwater rise was modeled for a SLR of $+2.00 \mathrm{~m}, 14.3$ percent of the nest sites were inundated (table 2.6). Similarly, the wave-driven inundation model (assuming unlimited seawater volumes and no infiltration) at $+2.00 \mathrm{~m}$ SLR exhibited a 22.9-percent loss in nest sites.

The same habitat classes were identified as potential nesting habitat (that is, habitat based on land cover classes, not survey data) for MABO (265.2 ha) and BRBO (262.1 ha), excluding the hard pan that was used by MABO only. Therefore, inundation risks to potential habitat for these two groundnesting boobies were almost equal. When incorporating wave-driven inundation, potential habitat loss (not shown in table 2.6) was less than current nest site losses for both species, as potential nesting habitat was distributed across the island, whereas current nest distribution was located primarily on the more vulnerable southern and eastern sides of the island (fig. 2.24). Of the potential ground-nesting booby habitat, 28.5 percent (MABO) and 29.3 percent (BRBO) were inundated with the wave-driven model, assuming unlimited seawater volumes and no infiltration at $+2.00 \mathrm{~m}$ SLR. Passive inundation models that incorporated rising groundwater predicted less inundation of current nest sites than potential habitat for MABO (14.8 percent) and similar levels of inundation for BRBO (15.6 percent).

The nesting distribution of Red-footed Booby (RFBO; $\mathrm{n}=539$ ) and Great Frigatebird (GRFR; n=1801) was nearly the same for each species in 2008 (fig. 2.25; USFWS data; Cornett and others, 2008), and generally included parts of the following land cover classes: tree/shrub, Casuarina equisetifolia (RFBO only), Pluchea indica, Tournefortia argentea, and mixed shrub. Under the passive modeling approach, we predicted no nesting habitat loss for these species, even at the highest SLR scenario considered. Under the wave-driven inundation model, 10.7 percent of the 2008 habitat was inundated under a SLR scenario of $+2.00 \mathrm{~m}$ (table 2.7). For the models that incorporated groundwater, minimal losses of habitat occurred until SLR exceeded $+1.00 \mathrm{~m}$, after which habitat losses for these species exceeded 50 percent. Similarly, the wave-driven inundation model with unlimited seawater volumes and no infiltration predicted no habitat loss for these species at SLR values less than or equal to $+1.00 \mathrm{~m}$, and extensive losses at SLR values of +1.50 and $+2.00 \mathrm{~m}$ (table 2.7 ).

Of the potential tree and shrub habitat used by these two species (39.3 ha, based on our land cover classification), less than 7 percent was inundated under both the passive inundation model and the wave-driven inundation model across all SLR values. However, when groundwater percolation was modeled (passive and wave-driven), inundation of potential RFBO and GRFR habitat exceeded 50 percent at $+1.00 \mathrm{~m}$ SLR and 60 percent at $+2.00 \mathrm{~m} \mathrm{SLR}$ (table 2.7). Across the entire range of SLR scenarios, a greater proportion of recent (that is, 2008) shrub habitat was inundated than potential shrub habitat, as the 2008 nesting distribution was concentrated in shrubs located in low-lying lakeside areas vulnerable to flooding (fig. 2.25).

Sooty Tern (SOTE) colonies for 2008, 2009, and 2010 ranged in area from 24.7 to 31.7 ha, with the spatial distribution of colonies varying widely from year to year (fig. 2.26). No losses in colony area were observed under a passive modeling approach for any year or SLR value (table 2.8). Likewise, the wave-driven inundation model exhibited virtually no losses in habitat, except for modest losses at the highest SLR scenario only. When seawater volumes were assumed to be unlimited, the wave-driven inundation model showed losses of 8.9 to 39.1 percent for the two highest values of SLR $(+1.50$ and $+2.00 \mathrm{~m})$; otherwise, at the lower SLR values no losses occurred. For the two models that incorporated rising groundwater, a wide range of habitat losses occurred, ranging from essentially zero to almost 30 percent, depending on the year and SLR scenario; in general, only the two highest SLR scenarios exhibited substantial losses of SOTE habitat under this modeling approach (table 2.8).

Of the potential SOTE habitat (265.2 ha), based on land cover class, 14.3 percent and 29.3 percent were inundated at $+2.00 \mathrm{~m}$ SLR using the passive model with rising groundwater and the wave-driven inundation model (assuming unlimited seawater volumes and no infiltration), respectively. In general, for the 3 years with data, potential habitat loss exceeded current nesting distribution loss, as potential habitat was distributed throughout the island including inundation-prone areas not recently used by SOTE (for example, low-lying interior areas subject to groundwater inundation and wave-driven seawater; table 2.8, fig. 2.26).

For two of the resident endangered birds, Laysan Teal and Laysan Finch, we observed patterns of potential nesting habitat loss that resembled the habitat-loss patterns for the other species (table 2.9, fig. 2.27). Both species rely heavily on bunch grass for nesting, and showed no losses in nesting habitat under a passive modeling approach and only modest losses in habitat with the wave-driven models at the highest SLR scenario $(+2.00$ $\mathrm{m})$ only. When considering unlimited seawater volumes and no infiltration, large losses in nesting habitat occurred at the two highest SLR scenarios (approximately 40 percent for both species). Similarly, when we incorporated groundwater into our models, substantial losses in nesting habitat occurred: 14.1 to 35.8 percent for both species at SLR scenarios of +1.00 to +2.00 $\mathrm{m}$ (table 2.9). The nesting habitat adjacent to the central lake was most vulnerable to inundation from unlimited volumes of wave-driven seawater and from rising groundwater (fig. 2.27). Although the two species nest in the same habitat classes, as ground nesters the Laysan Teal may be more vulnerable than the Laysan Finch to periodic inundation. 
Table 2.7. Inundated 2008 nesting habitat at Laysan Island (USFWS data; Cornett and others, 2008) for the Red-footed Booby (RFBO) and Great Frigatebird (GRFR) under five sea-level rise (SLR) scenarios and five modeling approaches:

(1) passive inundation, (2) dynamic wave-driven inundation, (3) dynamic wave-driven inundation with unlimited seawater volumes and no infiltration, (4) passive SLR including a comparable rise in groundwater, and (5) dynamic wave-driven inundation including a comparable rise in groundwater.

[Inundated habitat is expressed in area and percent change relative to the total habitat without inundation (listed in last row). Note that for the purposes of this study, we assumed the colony distribution from 2008 was representative of long-term spatial patterns. In this table, the shrubnesting habitat of GRFR and RFBO are equal . Abbreviations: USFWS, U.S. Fish and Wildlife Service; m, meters; ha, hectares; \%, percent]

\begin{tabular}{|c|c|c|c|c|}
\hline \multirow[b]{2}{*}{ Model } & \multirow[b]{2}{*}{$\begin{array}{c}\text { SLR } \\
\text { scenario }\end{array}$} & \multirow{2}{*}{$\begin{array}{c}\text { Total } \\
\text { inundation } \\
\text { area (ha) }\end{array}$} & \multicolumn{2}{|c|}{$\begin{array}{c}\text { Red-footed Booby and Great } \\
\text { Frigatebird }\end{array}$} \\
\hline & & & $\begin{array}{c}\text { Inundated } \\
2008 \text { habitat } \\
\text { (ha) }\end{array}$ & $\begin{array}{l}\% \text { change in } \\
2008 \text { habitat }\end{array}$ \\
\hline \multirow[t]{5}{*}{ Passive inundation } & $0.00 \mathrm{~m}$ & 0.4 & 0.0 & 0.0 \\
\hline & $+0.50 \mathrm{~m}$ & 3.1 & 0.0 & 0.0 \\
\hline & $+1.00 \mathrm{~m}$ & 6.7 & 0.0 & 0.0 \\
\hline & $+1.50 \mathrm{~m}$ & 10.8 & 0.0 & 0.0 \\
\hline & $+2.00 \mathrm{~m}$ & 15.5 & 0.0 & 0.0 \\
\hline \multirow[t]{5}{*}{ Dynamic wave-driven inundation } & $0.00 \mathrm{~m}$ & 6.1 & 0.0 & 0.0 \\
\hline & $+0.50 \mathrm{~m}$ & 10.5 & 0.0 & 0.0 \\
\hline & $+1.00 \mathrm{~m}$ & 15.5 & 0.0 & 0.0 \\
\hline & $+1.50 \mathrm{~m}$ & 22.1 & 0.0 & 0.0 \\
\hline & $+2.00 \mathrm{~m}$ & 57.9 & 1.6 & -10.7 \\
\hline Dynamic wave-driven inundation, & $0.00 \mathrm{~m}$ & 6.1 & 0.0 & 0.0 \\
\hline \multirow[t]{4}{*}{ unlimited seawater and no infiltration } & $+0.50 \mathrm{~m}$ & 10.5 & 0.0 & 0.0 \\
\hline & $+1.00 \mathrm{~m}$ & 15.5 & 0.0 & 0.0 \\
\hline & $+1.50 \mathrm{~m}$ & 115.8 & 10.3 & -69.6 \\
\hline & $+2.00 \mathrm{~m}$ & 136.2 & 10.6 & -71.1 \\
\hline \multirow[t]{5}{*}{ Passive + groundwater inundation } & $0.00 \mathrm{~m}$ & 0.4 & 0.0 & 0.0 \\
\hline & $+0.50 \mathrm{~m}$ & 13.8 & 0.1 & -1.0 \\
\hline & $+1.00 \mathrm{~m}$ & 40.8 & 8.1 & -54.5 \\
\hline & $+1.50 \mathrm{~m}$ & 70.2 & 10.2 & -68.4 \\
\hline & $+2.00 \mathrm{~m}$ & 92.6 & 10.2 & -69.0 \\
\hline \multirow[t]{5}{*}{ Dynamic wave-driven + groundwater inundation } & $0.00 \mathrm{~m}$ & 6.1 & 0.0 & 0.0 \\
\hline & $+0.50 \mathrm{~m}$ & 21.2 & 0.1 & -1.0 \\
\hline & $+1.00 \mathrm{~m}$ & 49.6 & 8.1 & -54.5 \\
\hline & $+1.50 \mathrm{~m}$ & 81.4 & 10.2 & -68.5 \\
\hline & $+2.00 \mathrm{~m}$ & 122.1 & 10.4 & -69.9 \\
\hline Total terrestrial habitat (without inundation) & & 337.8 & 14.9 & \\
\hline
\end{tabular}




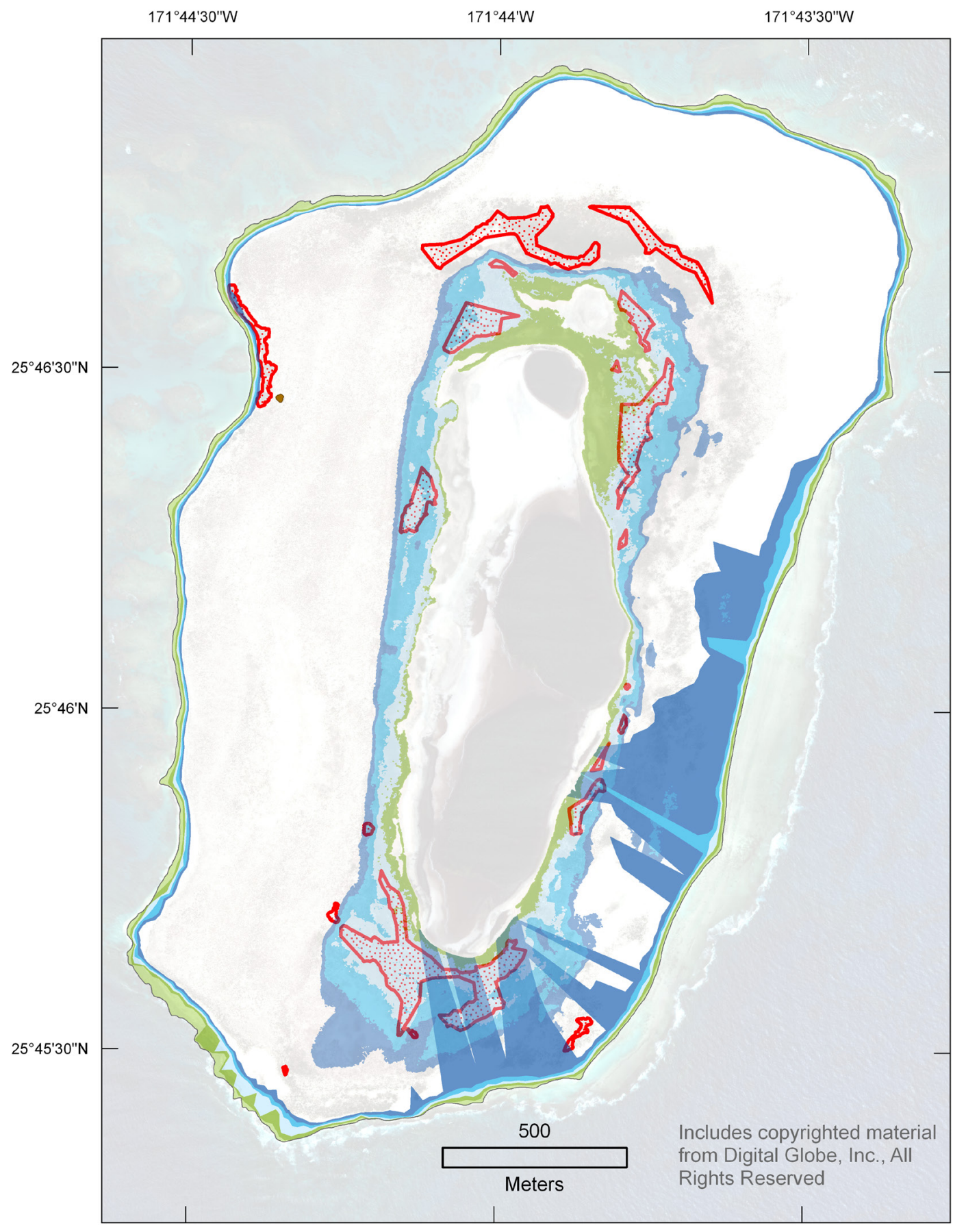

Overlay of 2008 Red-Footed Booby and
Great Frigatebird Nest Sites with
Combined Inundation Patterns
2008 nest sites
Red-footed Booby and Great Frigatebird
Red-footed Booby only
Inundation area by SLR scenario
$0.00 \mathrm{~m}$
$+0.50 \mathrm{~m}$
$+1.00 \mathrm{~m}$
$+1.50 \mathrm{~m}$
$+2.00 \mathrm{~m}$
Shoreline (mean sea level)

Figure 2.25. Overlay of the shrub-nesting areas for Red-footed Booby (RFBO) and Great Frigatebird (GRFR) at Laysan Island, with combined inundation from passive sea-level rise (SLR), wave-driven water levels, and rising groundwater. The combined background allows for a comparison of inundation extents from various models with the displayed colonies. Colony areas appear lighter when covered by inundation layers. A semi-transparent satellite image appears in the background (Digital Globe Inc., 2010). U.S. Fish and Wildlife Service (USFWS) biologists conducted a complete survey of these two species June 10-12, 2008, consolidating the data into 21 colonies covering 3.7 hectares (ha; USFWS data; Cornett and others, 2008). Both species occupied essentially the same nesting habitat. The nesting sites for these two species were found primarily near the central lake in locations above the wave-driven inundation zone until SLR reached +2.00 meters $(\mathrm{m})$. These nesting areas were vulnerable to rising lake levels, assuming groundwater were to rise with sea level. 
Table 2.8. Inundated colony areas at Laysan Island (USFWS data; Cornett and others, 2008; Boyd and others, 2009; Hammond and others, 2010) for Sooty Tern under five sea-level rise (SLR) scenarios and five modeling approaches: (1) passive inundation, (2) dynamic wave-driven inundation, (3) dynamic wave-driven inundation with unlimited seawater volumes and no infiltration, (4) passive SLR including a comparable rise in groundwater, and (5) dynamic wave-driven inundation including a comparable rise in groundwater.

[Inundated habitat is expressed in area and percent change relative to the total habitat without inundation (listed by year in last row). Abbreviations: USFWS, U.S. Fish and Wildlife Service; m, meters; ha, hectares; \%, percent]

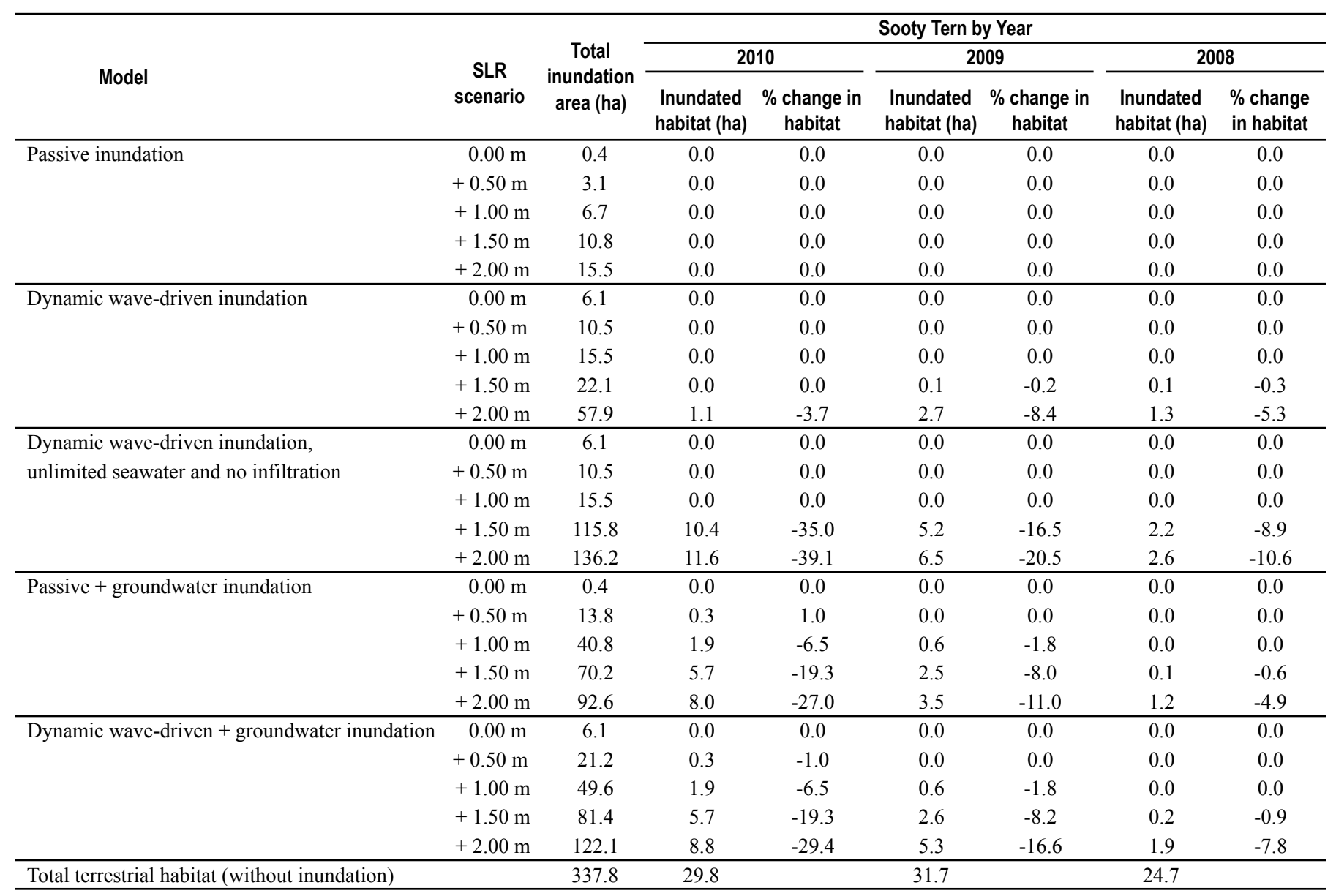




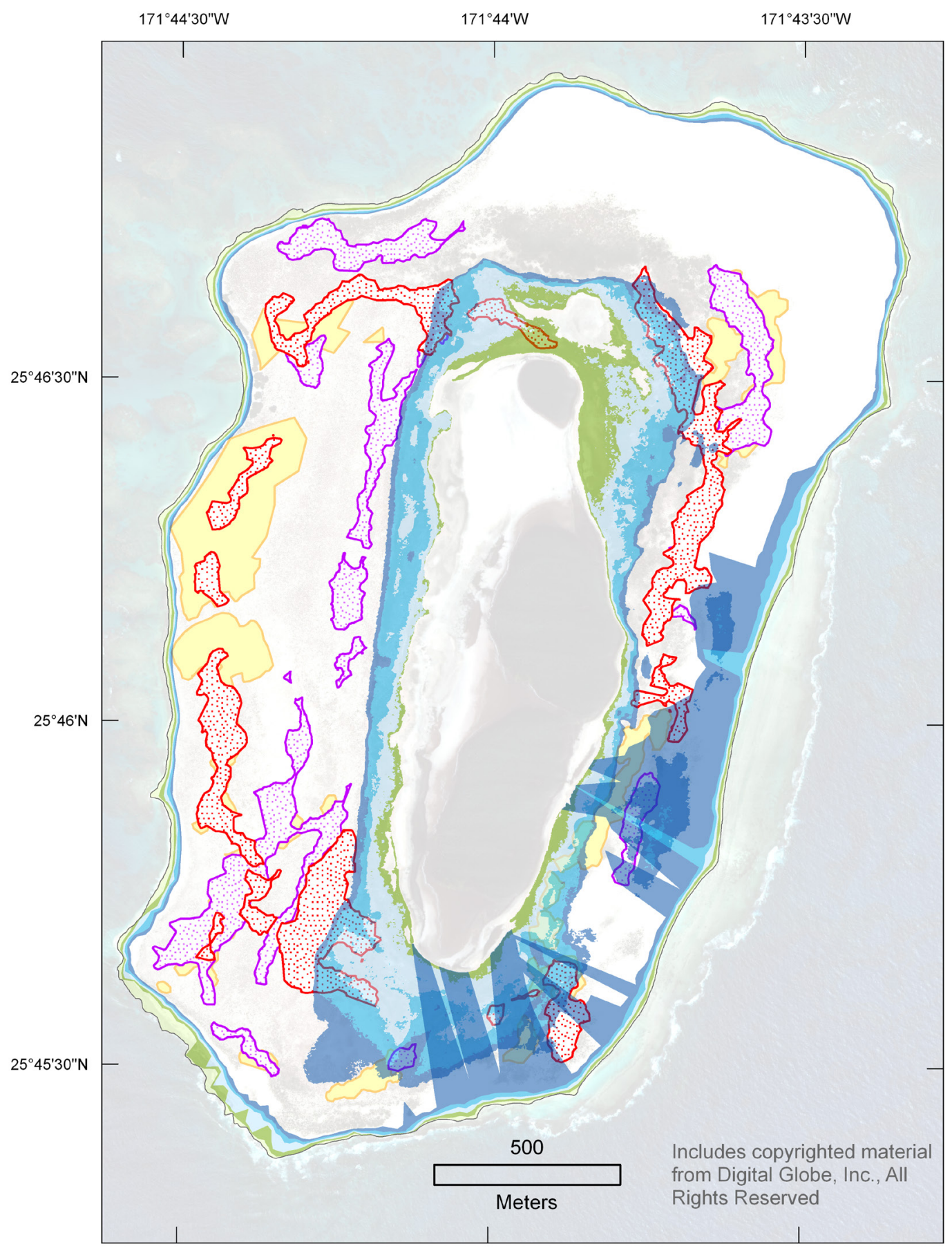

Overlay of 2008-2010 Sooty Tern Colonies with Combined Inundation Patterns

Sooty Tern colonies by year

Sooty Tern colonies 2010

Sooty Tern colonies 2009

Sooty Tern colonies 2008

Inundation area by SLR scenario

$0.00 \mathrm{~m}$

$+0.50 \mathrm{~m}$

$+1.00 \mathrm{~m}$

$+1.50 \mathrm{~m}$

$+2.00 \mathrm{~m}$

Shoreline (mean sea level)

Figure 2.26. Overlay of Sooty Tern (SOTE) colonies at Laysan Island with combined inundation from passive sea-level rise (SLR), wave-driven water levels, and rising groundwater. The combined background allows for a comparison of inundation extents from various models with the displayed colony locations. Colony locations appear lighter when covered by inundation layers. A semitransparent satellite image appears in the background (Digital Globe Inc., 2010). Biologists surveyed SOTE colonies in 2008, 2009, and 2010 U.S. Fish and Wildlife Service data; Cornett and others, 2008; Boyd and others, 2009; Hammond and others, 2010). Large annual differences in colony locations existed, as SOTE exhibited a wider range of nesting habitat types and less nest-site fidelity than other seabird species. Less than 7 percent of the colonies were located in the inundation zone for SLR scenarios +1.00 meters $(m)$. Even under worst-case assumptions (that is, a SLR of $+2.00 \mathrm{~m}$ with wave-driven and groundwater inundation), only $7.8,16.6$, and 29.4 percent of the colony locations for 2008, 2009, and 2010 respectively, were located in the inundation zone. Given SOTE's adaptability, it is likely that colonies would move to available habitat during extreme inundation events. 
Table 2.9. Inundated potential nesting habitat at Laysan Island for Laysan Teal and Laysan Finch under five sea-level rise (SLR) scenarios and five modeling approaches: (1) passive inundation, (2) dynamic wave-driven inundation, (3) dynamic wave-driven inundation with unlimited seawater volumes and no infiltration, (4) passive SLR including a comparable rise in groundwater, and (5) dynamic wave-driven inundation including a comparable rise in groundwater.

[Inundated habitat is expressed in area and percent change relative to the total habitat without inundation (listed in last row). Abbreviations: m, meters; ha, hectares; \%, percent]

\begin{tabular}{|c|c|c|c|c|}
\hline \multirow[b]{2}{*}{ Model } & \multirow[b]{2}{*}{$\begin{array}{c}\text { SLR } \\
\text { scenario }\end{array}$} & \multirow{2}{*}{$\begin{array}{c}\text { Total } \\
\text { inundation } \\
\text { area (ha) }\end{array}$} & \multicolumn{2}{|c|}{ Laysan Teal and Laysan Finch } \\
\hline & & & $\begin{array}{l}\text { Inundated } \\
\text { habitat (ha) }\end{array}$ & $\begin{array}{l}\% \text { change in } \\
\text { habitat }\end{array}$ \\
\hline \multirow[t]{5}{*}{ Passive inundation } & $0.00 \mathrm{~m}$ & 0.4 & 0.0 & 0.0 \\
\hline & $+0.50 \mathrm{~m}$ & 3.1 & 0.0 & 0.0 \\
\hline & $+1.00 \mathrm{~m}$ & 6.7 & 0.0 & 0.0 \\
\hline & $+1.50 \mathrm{~m}$ & 10.8 & 0.0 & 0.0 \\
\hline & $+2.00 \mathrm{~m}$ & 15.5 & 0.0 & 0.0 \\
\hline \multirow[t]{5}{*}{ Dynamic wave-driven inundation } & $0.00 \mathrm{~m}$ & 6.1 & 0.0 & 0.0 \\
\hline & $+0.50 \mathrm{~m}$ & 10.5 & 0.0 & 0.0 \\
\hline & $+1.00 \mathrm{~m}$ & 15.5 & 0.0 & 0.0 \\
\hline & $+1.50 \mathrm{~m}$ & 22.1 & 0.2 & -0.1 \\
\hline & $+2.00 \mathrm{~m}$ & 57.9 & 12.8 & -7.4 \\
\hline \multirow{5}{*}{$\begin{array}{l}\text { Dynamic wave-driven inundation, } \\
\text { unlimited seawater and no infiltration }\end{array}$} & $0.00 \mathrm{~m}$ & 6.1 & 0.0 & 0.0 \\
\hline & $+0.50 \mathrm{~m}$ & 10.5 & 0.0 & 0.0 \\
\hline & $+1.00 \mathrm{~m}$ & 15.5 & 0.0 & 0.0 \\
\hline & $+1.50 \mathrm{~m}$ & 115.8 & 66.0 & -38.3 \\
\hline & $+2.00 \mathrm{~m}$ & 136.2 & 71.7 & -41.6 \\
\hline \multirow[t]{5}{*}{ Passive + groundwater inundation } & $0.00 \mathrm{~m}$ & 0.4 & 0.0 & 0.0 \\
\hline & $+0.50 \mathrm{~m}$ & 13.8 & 5.4 & -3.1 \\
\hline & $+1.00 \mathrm{~m}$ & 40.8 & 24.2 & -14.1 \\
\hline & $+1.50 \mathrm{~m}$ & 70.2 & 45.8 & -26.6 \\
\hline & $+2.00 \mathrm{~m}$ & 92.6 & 56.6 & -32.9 \\
\hline \multirow[t]{5}{*}{ Dynamic wave-driven + groundwater inundation } & $0.00 \mathrm{~m}$ & 6.1 & 0.0 & 0.0 \\
\hline & $+0.50 \mathrm{~m}$ & 21.2 & 5.4 & -3.1 \\
\hline & $+1.00 \mathrm{~m}$ & 49.6 & 24.2 & -14.1 \\
\hline & $+1.50 \mathrm{~m}$ & 81.4 & 45.9 & -26.7 \\
\hline & $+2.00 \mathrm{~m}$ & 122.1 & 61.6 & -35.8 \\
\hline Total terrestrial habitat (without inundation) & & 337.8 & 172.2 & \\
\hline
\end{tabular}




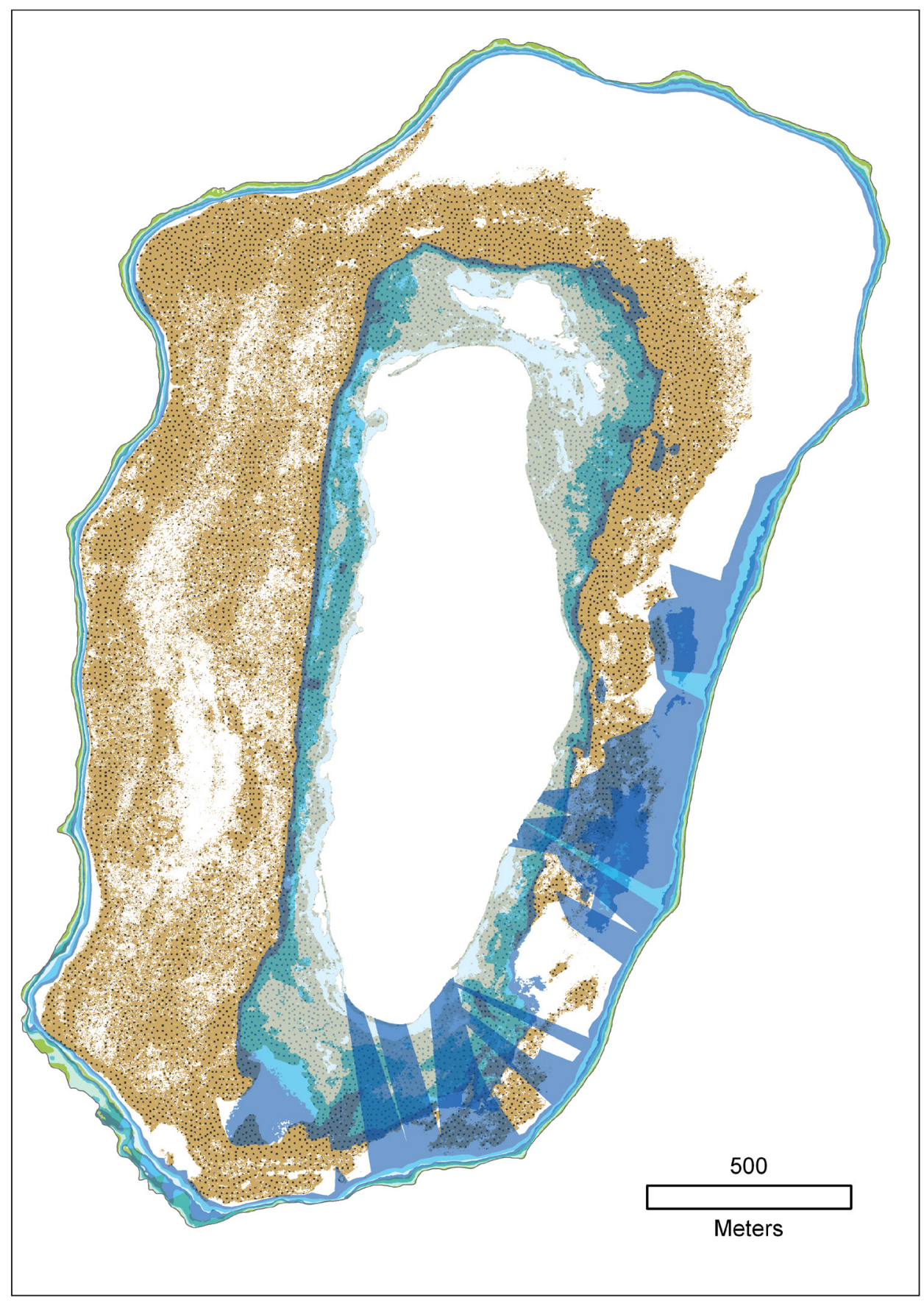

\section{Overlay of Laysan Teal and Laysan Finch Nesting Habitat with Combined Inundation Patterns}

Laysan Teal and Laysan Finch nesting habitat Inundation area by SLR scenario $0.00 \mathrm{~m}$

$+0.50 \mathrm{~m}$

$+1.00 \mathrm{~m}$

$+1.50 \mathrm{~m}$

$+2.00 \mathrm{~m}$

Shoreline (mean sea level)

Figure 2.27. Overlay of Laysan Teal (LADU) and Laysan Finch (LAFI) potential nesting habitat at Laysan Island, with combined inundation from passive sea-level rise (SLR), wave-driven water levels, and rising groundwater. The combined background allows for a comparison of inundation extents from various models with the displayed nesting habitat. By depicting inundation extent with semi-transparent symbols, the underlying nesting habitat remains visible, although may appear slightly lighter. Nesting habitat was determined from land cover maps and previous habitat studies (Reynolds, 2004; Reynolds and others, 2007). No losses in nesting habitat occurred under a passive modeling approach and only modest losses occurred with the wave-driven models at the highest SLR scenario (+2.00 meters, $\mathrm{m}$ ) only. When considering unlimited seawater volumes and no infiltration, losses in nesting habitat were approximately 40 percent for both species at the two highest SLR scenarios. Similarly, when we incorporated groundwater into our models, substantial losses occurred (14.1 to 35.8 percent) for SLR scenarios greater than or equal to $+1.00 \mathrm{~m}$. The nesting habitat adjacent to the central lake was the most vulnerable to inundation from either unlimited volumes of wave-driven seawater or rising groundwater. 
Table 2.10. Life-history traits (nests/year, breeding season, nest site fidelity, etc.) for 20 avian species on Laysan Island, used to evaluate vulnerability to inundation.

\begin{tabular}{|c|c|c|c|c|c|c|c|c|}
\hline \multirow[b]{2}{*}{ Species } & \multicolumn{6}{|c|}{ Adaptability } & \multicolumn{2}{|c|}{ Vulnerability } \\
\hline & Nesting frequency & Reclutch ability & $\begin{array}{l}\text { Maximum } \\
\text { young per year }\end{array}$ & $\begin{array}{l}\text { Nest site } \\
\text { fidelity }\end{array}$ & $\begin{array}{l}\text { Flexibility in habitat } \\
\text { use }\end{array}$ & $\begin{array}{l}\text { Inter-atoll dispersal } \\
\text { ability }\end{array}$ & $\begin{array}{l}\text { Overlap with peak } \\
\text { storm season }\end{array}$ & Nest proximity to inundation \\
\hline Black-footed Albatross ${ }^{1}$ & Annual to Alternate & No & 1 & High & High & Yes & Breeding, peak & High (coastal) \\
\hline Laysan Albatross $^{2}$ & Annual to Alternate & No & 1 & High & High & Yes & Breeding, peak & High (lake) \\
\hline Bonin Petrel $^{3}$ & Annual & No & 1 & High & Moderate & Yes & Breeding, peak & Unknown \\
\hline Bulwer's Petrel $^{4}$ & Annual & No & 1 & High & Moderate & Yes & Not present & Unknown \\
\hline Wedge-tailed Shearwater $^{5}$ & Annual to Alternate & No & 1 & High & Moderate & Yes & Not present & Unknown \\
\hline Christmas Shearwater ${ }^{6}$ & Annual to Alternate & No & 1 & High & High & Yes & Adults, off-peak & Unknown \\
\hline Tristram's Storm-petrel ${ }^{7}$ & Annual to Alternate & No & 1 & High & Moderate & Yes & Breeding, peak & High (lake) \\
\hline Red-tailed Tropicbird ${ }^{8}$ & Annual to Alternate & Yes & 1 & High & Moderate & Yes & Breeding, off-peak & Unknown \\
\hline Masked Booby ${ }^{9}$ & Annual & Yes & 1 & Low & Moderate & Yes & Breeding, aseasonal & Moderate (coastal) \\
\hline Brown Booby $^{10}$ & Annual & Yes & 1 & Moderate & Moderate & Yes & Breeding, off-peak & High (coastal) \\
\hline Red-footed Booby ${ }^{11}$ & Annual & Yes & 1 & Moderate & Low & Yes & Breeding, off-peak & High (lake) \\
\hline Great Frigatebird $^{12}$ & Annual to Alternate & Yes & 1 & Low & Low & Yes & Breeding, peak & High (lake) \\
\hline Gray-backed Tern ${ }^{13}$ & Annual to Alternate & Yes & 1 & Moderate & Moderate & Yes & Breeding, peak & *Moderate (lake and coastal) \\
\hline Sooty Tern ${ }^{14}$ & Annual & Yes & 1 & Low & Moderate & Yes & Adults, off-peak & Low (lake and coastal) \\
\hline Brown Noddy ${ }^{15}$ & Annual & Yes & 1 & Moderate & High & Yes & Breeding, aseasonal & Unknown \\
\hline Black Noddy $^{16}$ & Annual to Biannual & Yes & 2 & Moderate & Low & Yes & Breeding, aseasonal & *Moderate (lake and coastal) \\
\hline White Tern ${ }^{17}$ & Annual to Biannual & Yes & 2 & High & Moderate & Yes & Breeding, aseasonal & *Moderate (lake and coastal) \\
\hline Laysan Teal $^{18}$ & Annual to Alternate & Yes & 6 & Moderate $^{\mathrm{a}}$ & High & No & Breeding, aseasonal & Moderate (lake and coastal) \\
\hline Laysan Finch ${ }^{19}$ & Annual to Biannual & Yes & 4 & Moderate $^{\mathrm{a}}$ & Moderate & No & Adults & Low (lake and coastal) \\
\hline Nihoa Millerbird ${ }^{\mathrm{b}, 20}$ & Annual to Biannual & Unknown & Unknown & High & Moderate & No & Breeding, peak & Unknown \\
\hline
\end{tabular}

"Laysan Teal and Finch are territorial nesters, not colonial breeders. ${ }^{b}$ Data for the recently (September 2011) translocated population of Nihoa Millerbird, derived from data from Nihoa. ${ }^{~}$ Rice and Kenyon (1962), Robbins (1966). ${ }^{2}$ Rice and Kenyon (1962), Fisher (1971). ${ }^{3}$ Grant and others (1983), Moore (2009). ${ }^{4}$ Amerson and Shelton (1976), Megyesi and O'Daniel (1997), Mougin 1990 in Megyesi and O'Daniel (1997). ${ }^{5}$ Ely and Clapp (1973), Shallenberger 1973 in Whittow (1997), Warham(1996), Whittow (1997), Dunlop and others (2002). ${ }^{6}$ Ely and Clapp (1973), Naughton (1982), Warham (1996), Seto (2001). ${ }^{7}$ Marks and Leasure (1992), Slotterback (2002), McClelland and others (2008). ${ }^{8}$ Fleet (1972), Amerson and Shelton (1976), Schreiber and Schreiber (2009). ${ }^{9}$ Ely and Clapp (1973), Nelson (1978), Grace and Anderson (2009), Kepler 1969 in Grace and Anderson (2009). ${ }^{10}$ Amerson (1971), Woodward (1972), Ely and Clapp (1973), Clapp and Wirtz (1975), Schreiber and Norton (2002), Tershy 1998 in Schreiber and Norton (2002). "Ely and Clapp (1973), Nelson (1978), Schreiber and others (1996), Citta and others (2007). ${ }^{12}$ Dearborn and Anders, personal commun. in Metz and Shreiber (2002), Ely and Clapp (1973), Metz and Schreiber (2002), Citta and others (2007), ${ }^{13}$ Ely and Clapp (1973), Amerson and Shelton (1976), Mostello and others (2000). ${ }^{4}$ Ely and Clapp (1973), Feare (1976), Saliva and Burger (1989), Schreiber and others (2002), Cornett and others (2008), Boyd and others (2009), Hammond and others (2010), USFWS data. ${ }^{15}$ Ely and Clapp (1973), Chardine and Morris (1996), Megyesi and Griffin (1996). ${ }^{16}$ Ely and Clapp (1973), Gauger (1999). ${ }^{17}$ Ashmole (1968), Ely and Clapp (1973), Niethammer and Patrick (1998). ${ }^{18}$ Moulton and Marshall (1996), Reynolds (2002), Reynolds (2004). Laysan Teal are territorial nesters, not colonial breeders. ${ }^{19}$ Morin (1991), Morin (1992a, b), Morin and Conant (2002). Laysan Finch are territorial nesters, not colonial breeders. ${ }^{20}$ Morin and others (1997), USFWS (2011b). *Not modeled spatially, based on potential nesting habitat distribution.[Abbreviations: USFWS, U.S. Fish and Wildlife Service] 
a.

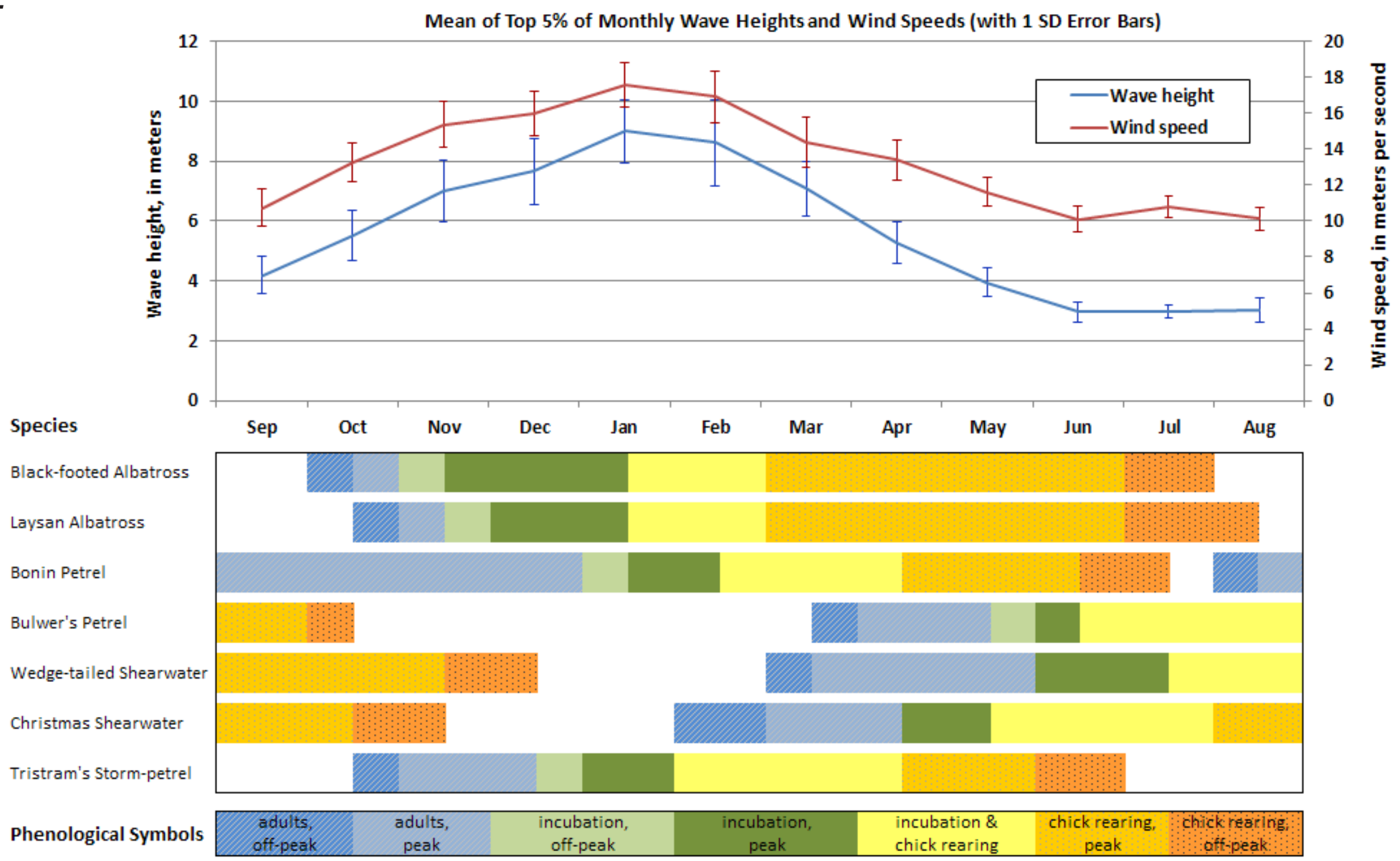

Figure 2.28. Breeding phenology of 20 birds at Laysan Island compared to the monthly mean of the top 5 percent of wave heights and wind speeds (with 1-standard deviation error bars). Wave height and wind speed data were acquired from the U.S. Army Corps of Engineers (2011) Wave Information System for a location (28 $00^{\prime} \mathrm{N}, 174^{\circ} 00^{\prime} \mathrm{W}$ ) approximately 375 kilometers $(\mathrm{km})$ northwest of Laysan Island. The groups include (a) Procellariiformes (Black-footed Albatross, Laysan Albatross, Bonin Petrel, Bulwer's Petrel, Wedge-tailed Shearwater, Christmas Shearwater, and Tristram's Storm-petrel) that nest above ground, in crevices, or in subterranean burrows; (b) other ground-nesting species (Red-tailed Tropicbird, Masked Booby, Brown Booby, Gray-backed Tern, Sooty Tern, Brown Noddy, and Laysan Teal) that nest on the ground, on low vegetation, or under vegetation; and (c) tree- and shrub-nesting species (Red-footed Booby, Great Frigatebird, Black Noddy, White Tern, Laysan Finch, and Nihoa Millerbird). Breeding stage was divided into seven periods (listed across bottom row) based on adult presence and reproductive investment. Abbreviations: \%, percent. 


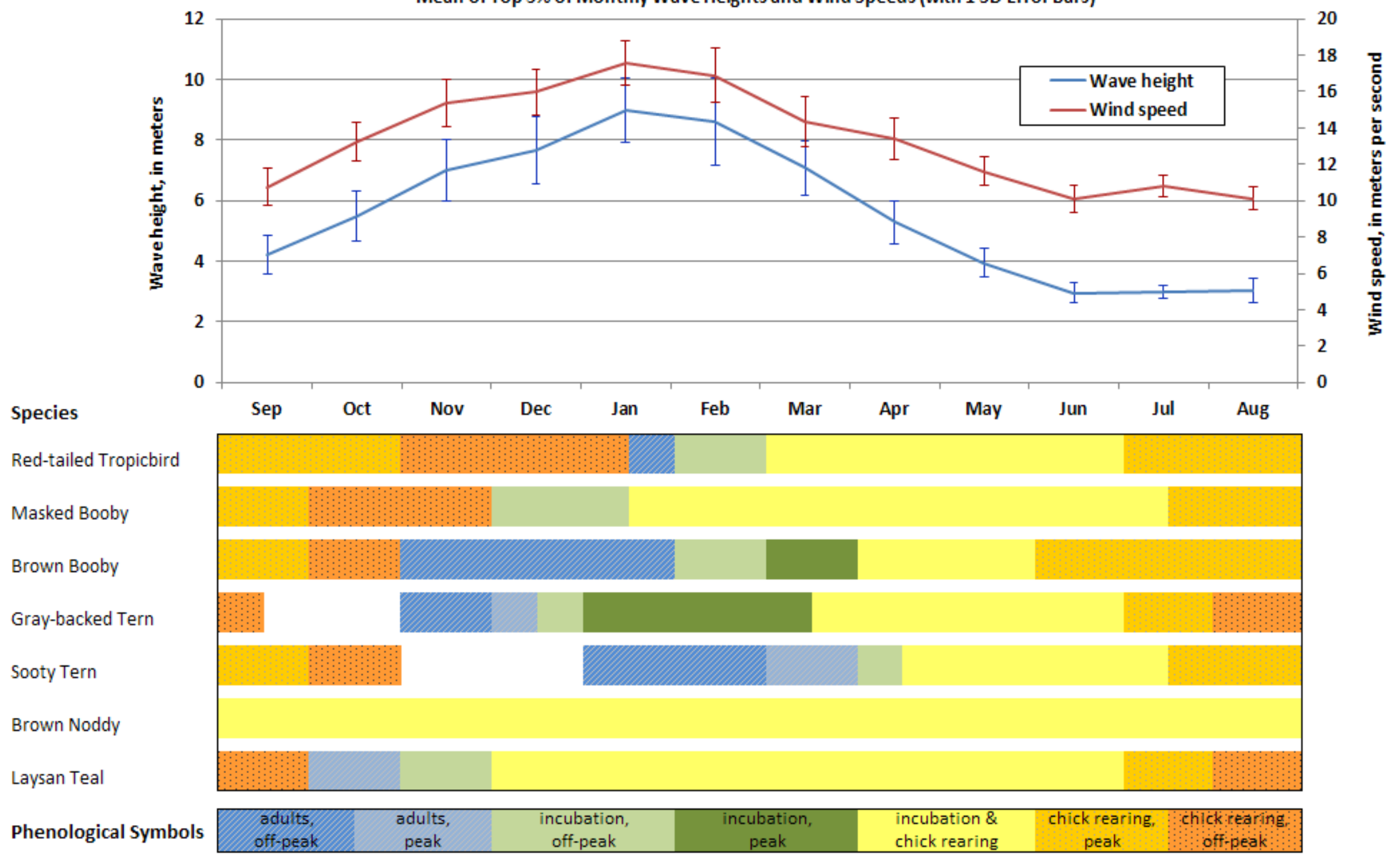

Figure 2.28. Breeding phenology of 20 birds at Laysan Island compared to the monthly mean of the top 5 percent of wave heights and wind speeds (with 1-standard deviation error bars). Wave height and wind speed data were acquired from the U.S. Army Corps of Engineers (2011) Wave Information System for a location (28 $00^{\prime} \mathrm{N}, 174^{\circ} 00^{\prime} \mathrm{W}$ ) approximately 375 kilometers $(\mathrm{km})$ northwest of Laysan Island. The groups include (a) Procellariiformes (Black-footed Albatross, Laysan Albatross, Bonin Petrel, Bulwer's Petrel, Wedge-tailed Shearwater, Christmas Shearwater, and Tristram's Storm-petrel) that nest above ground, in crevices, or in subterranean burrows; (b) other ground-nesting species (Red-tailed Tropicbird, Masked Booby, Brown Booby, Gray-backed Tern, Sooty Tern, Brown Noddy, and Laysan Teal) that nest on the ground, on low vegetation, or under vegetation; and (c) tree- and shrub-nesting species (Red-footed Booby, Great Frigatebird, Black Noddy, White Tern, Laysan Finch, and Nihoa Millerbird). Breeding stage was divided into seven periods (listed across bottom row) based on adult presence and reproductive investment. Abbreviations: \%, percent.-Continued 


\section{Species}

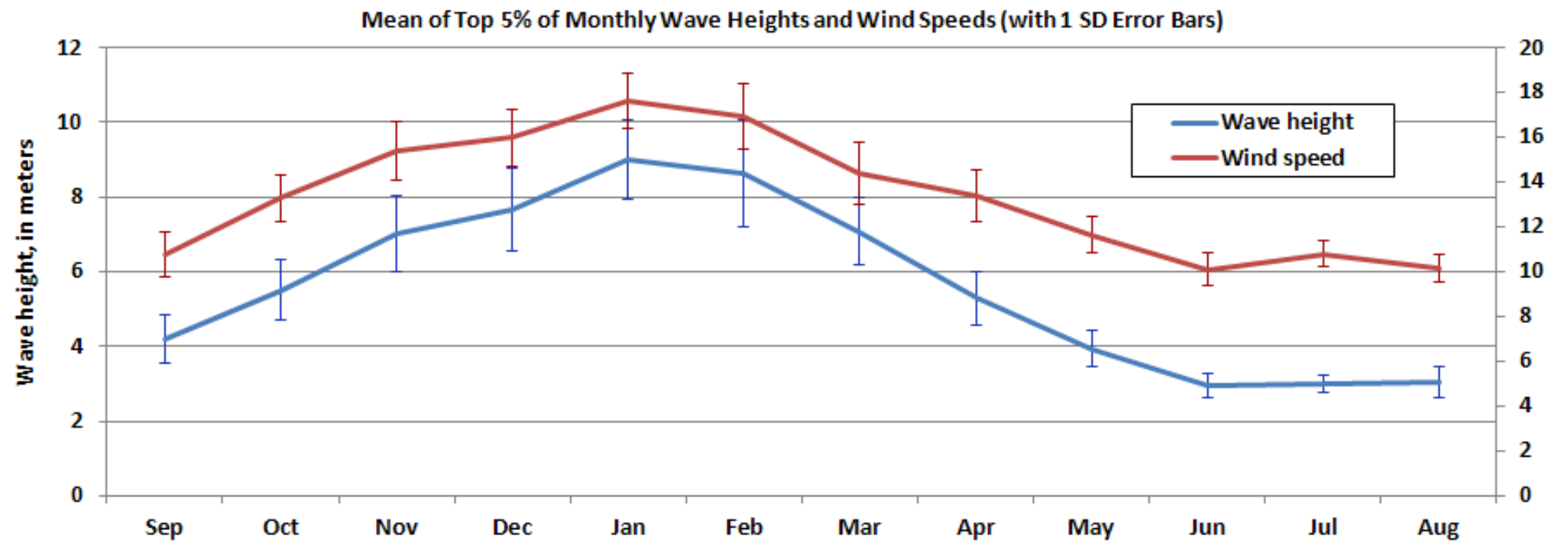

Red-footed Booby

Great Frigatebird

Black Noddy

White Tern

Laysan Finch

Nihoa Millerbird

Phenological Symbols

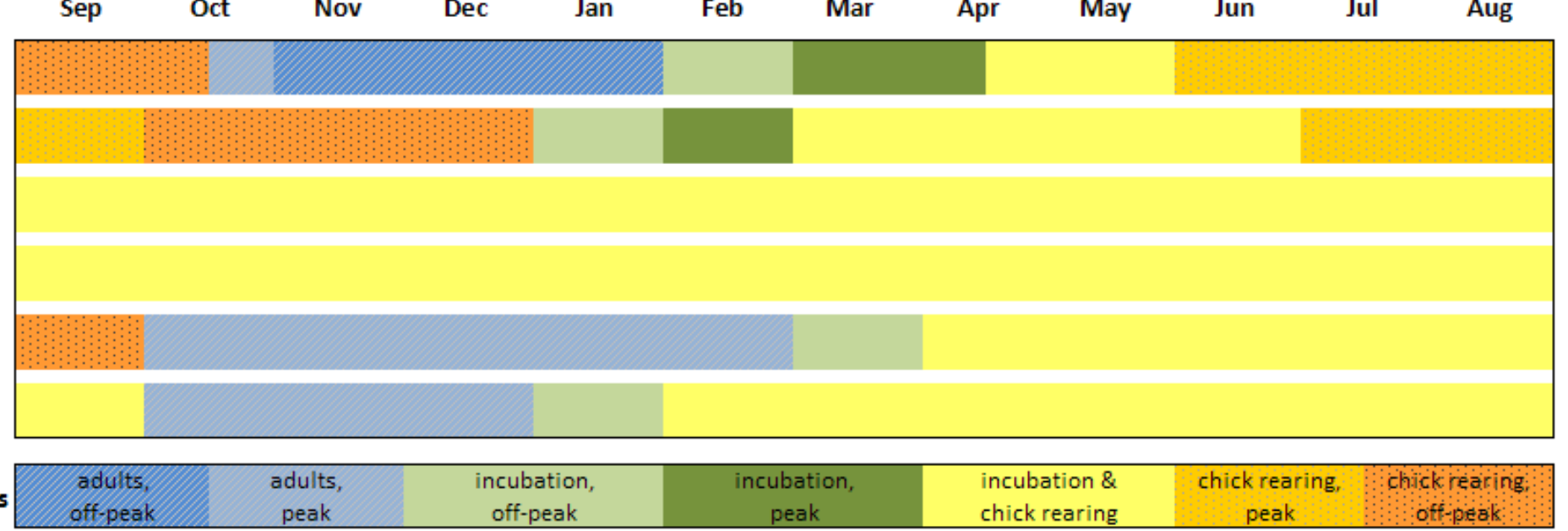

Figure 2.28. Breeding phenology of 20 birds at Laysan Island compared to the monthly mean of the top 5 percent of wave heights and wind speeds (with 1-standard deviation error bars). Wave height and wind speed data were acquired from the U.S. Army Corps of Engineers (2011) Wave Information System for a location (28 $00^{\prime} \mathrm{N}, 174^{\circ} 00^{\prime} \mathrm{W}$ ) approximately 375 kilometers $(\mathrm{km})$ northwest of Laysan Island. The groups include (a) Procellariiformes (Black-footed Albatross, Laysan Albatross, Bonin Petrel, Bulwer's Petrel, Wedge-tailed Shearwater, Christmas Shearwater, and Tristram's Storm-petrel) that nest above ground, in crevices, or in subterranean burrows; (b) other ground-nesting species (Red-tailed Tropicbird, Masked Booby, Brown Booby, Gray-backed Tern, Sooty Tern, Brown Noddy, and Laysan Teal) that nest on the ground, on low vegetation, or under vegetation; and (c) tree- and shrub-nesting species (Red-footed Booby, Great Frigatebird, Black Noddy, White Tern, Laysan Finch, and Nihoa Millerbird). Breeding stage was divided into seven periods (listed across bottom row) based on adult presence and reproductive investment. Abbreviations: \%, percent.-Continued 


\section{Vulnerability of Breeding Birds to Storm Conditions}

Seasonal overlap of breeding birds with the period of highest wave energy and wind speed varied among species (table 2.10, fig. 2.28a-c). Of the seven Procellariiformes, the peak breeding period of four species (Black-footed Albatross, Laysan Albatross, Bonin Petrel, and Tristram's Stormpetrel) overlapped the period of highest wave energy (Dec.Feb.) each year (fig. 2.28a). During this period, adults of these four species were generally present incubating eggs or brooding young chicks, making both adults and nest contents vulnerable to storm impacts. Of the winter-nesting Procellariiformes, Bonin Petrel and Tristram's Storm-petrel nest in underground burrows and may be particularly vulnerable to flooding from storm wave inundation or heavy rainfall. Life-history traits for species in this group (constrained to annual or alternate nesting frequency, inability to renest if nest contents are lost, and high nest-site fidelity) indicated that they have little capacity to adapt rapidly to direct loss of nests or nesting habitat (table 2.10).

For other ground-nesting species, the coincidence of breeding season and high-wave energy was greatest for Gray-backed Tern (fig. 2.28b). Red-tailed Tropicbird and Masked Booby, as aseasonal breeders with limited egg-laying during the period of high-wave energy, exhibited less vulnerability to winter storm conditions (fig. 2.28b). For Brown Booby and Sooty Tern, peak nesting season typically did not coincide with the period of highest wave energy. Since Brown Noddy nest throughout the year, only a limited portion of their reproductive period overlapped with winter storm conditions (fig. 2.28b). For Laysan Teal, a year-round resident with a variable breeding season, overlap between breeding season and high-wave energy (Dec.-Feb.) is likely to occur in some but not all years (fig. 2.28b). Since Laysan Teal nested in widely distributed sites rather than in colonies, inundation risk for this species was dispersed spatially. In general, ground-nesting tern species display the greatest potential to renest after nest loss and show lower nest-site fidelity compared to Procellariiformes and some other species (table 2.10).

Tree- and shrub-nesting species at Laysan were present throughout the year and typically have protracted breeding seasons (fig. 2.28c). Generally more vulnerable to high winds than to inundation, the coincidence of breeding season with winter-storm conditions may result in nest loss for these species. Egg laying and incubation may occur during the period of highwave energy and high wind speeds for at least some part of the population of each shrub-nesting species (fig. 2.28c). Since the shrub-nesting species of Laysan are the least flexible in their nesting habitat (table 2.10), they may be the least capable of adapting to losses of nesting habitat.

Table 2.11. Population parameter estimates for the Gompertz model ( $c=$ the strength of density dependence, $K$ = carrying capacity) with standard deviations (SD) and credible intervals (Cl) for three seabird species (BFAL, Black-footed Albatross; LAAL, Laysan Albatross; and RFBO, Red-footed Booby) and two endangered resident birds (LADU, Laysan Teal; and LAFI, Laysan Finch) with abundance time-series data at Laysan Island.

[Observation error $(\tau)$ and process variation $(\sigma)$ are not reported for species if the estimate of $c$ is close to zero (Dennis and others, 2010). High variation in abundance between years prevented reliable $\tau$ and $\sigma$ estimates for most species]

\begin{tabular}{lccccccc}
\hline Species & Years & Parameter & Mean & SD & $\mathbf{2 . 5 \%} \mathbf{C l}$ & Median & $\mathbf{9 7 . 5 \%} \mathbf{C l}$ \\
\hline *BFAL & $2001-2011$ & $c$ & -0.44 & \pm 0.37 & -1.1 & 0.46 & 0.38 \\
& & $K$ & 48,057 & $\pm 5,918$ & - & - & - \\
\hline *LAAL & $2001-2011$ & $c$ & 0.02 & \pm 0.02 & 0.92 & 0.96 & 1.01 \\
& & $K$ & 284,491 & $\pm 43,886$ & - & - & - \\
\hline *RFBO & $1996-2011$ & $c$ & 0.04 & \pm 0.55 & -1.01 & 0.15 & 0.92 \\
& & $K$ & 1395 & \pm 518 & - & - & - \\
\hline LADU & $1993-2010$ & $c$ & 0.68 & \pm 0.10 & 0.45 & 0.69 & 0.87 \\
& & $\sigma$ & 0.11 & \pm 0.04 & 0.03 & 0.11 & 0.2 \\
& & $\tau$ & 0.07 & \pm 0.04 & 0.03 & 0.06 & 0.16 \\
& & $K$ & 434 & \pm 72 & - & - & - \\
* LAFI & $1983-2011$ & $c$ & -0.18 & \pm 0.51 & -1.04 & -0.011 & 0.76 \\
& & $K$ & 10,580 & $\pm 3,513$ & - & - & - \\
\hline
\end{tabular}

\footnotetext{
* There is little correlation between population sizes among years for all species besides Laysan Teal, preventing reliable estimates of parameters. Future abundance monitoring for Laysan bird populations could be improved with independent replicate counts, especially for the Red-footed Booby (Citta and others, 2007) and Laysan Finch. Replicate counts (either using maximum mean incubation count or other methods, see Dearborn and Anders, 2006) are needed to better clarify sampling variance and the other parameters for Laysan Finch (see Citta and others, 2007; Dennis and others, 2010).
} 
(a) Black-footed Albatross

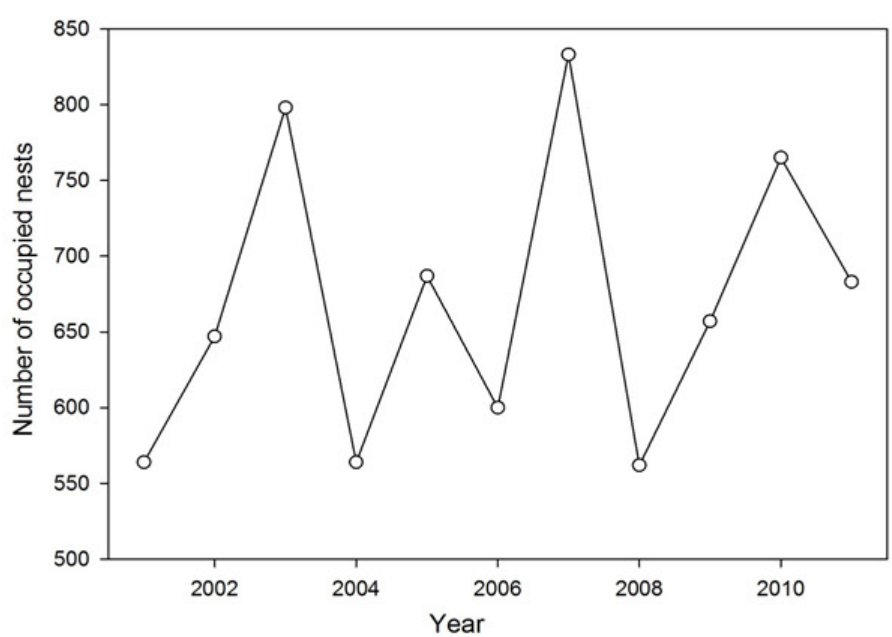

(c) Red-footed Booby

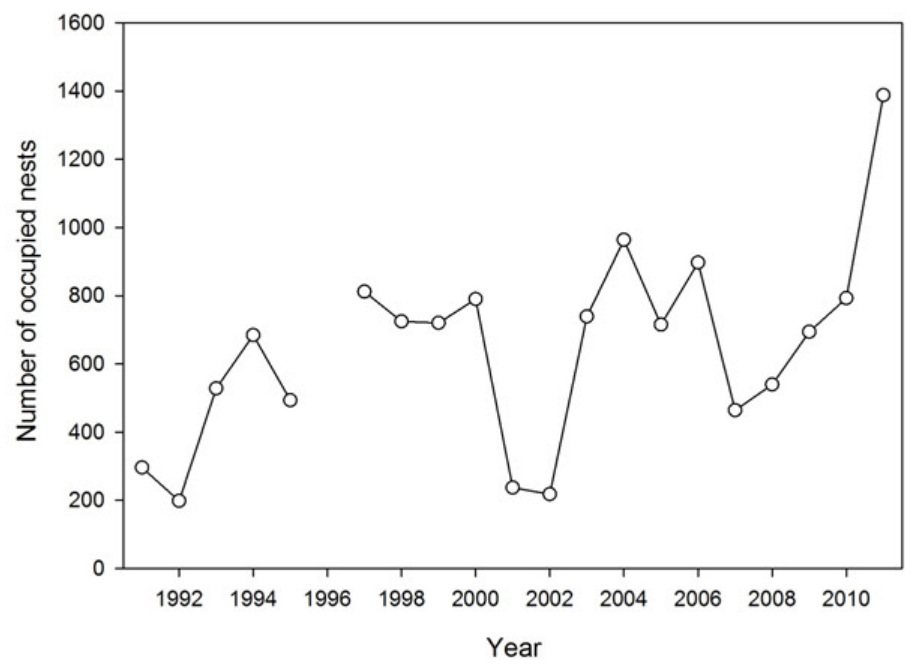

(e) Laysan Finch

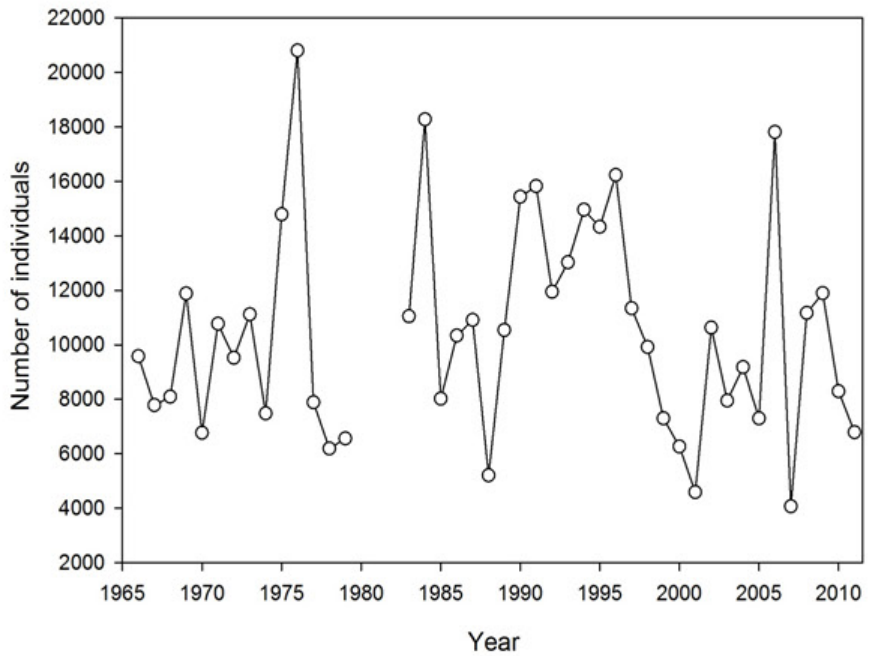

(b) Laysan Albatross

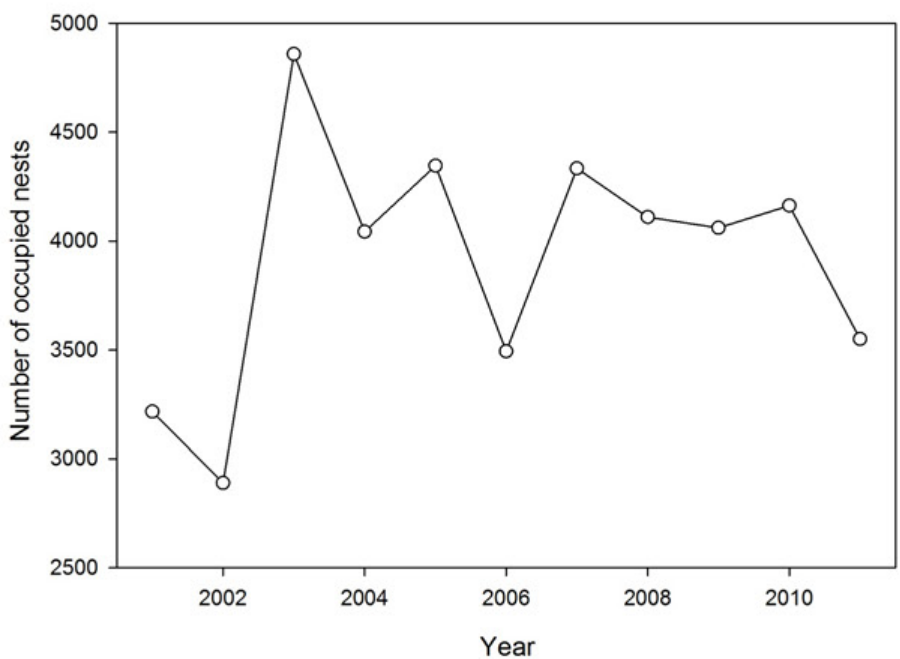

(d) Laysan Teal

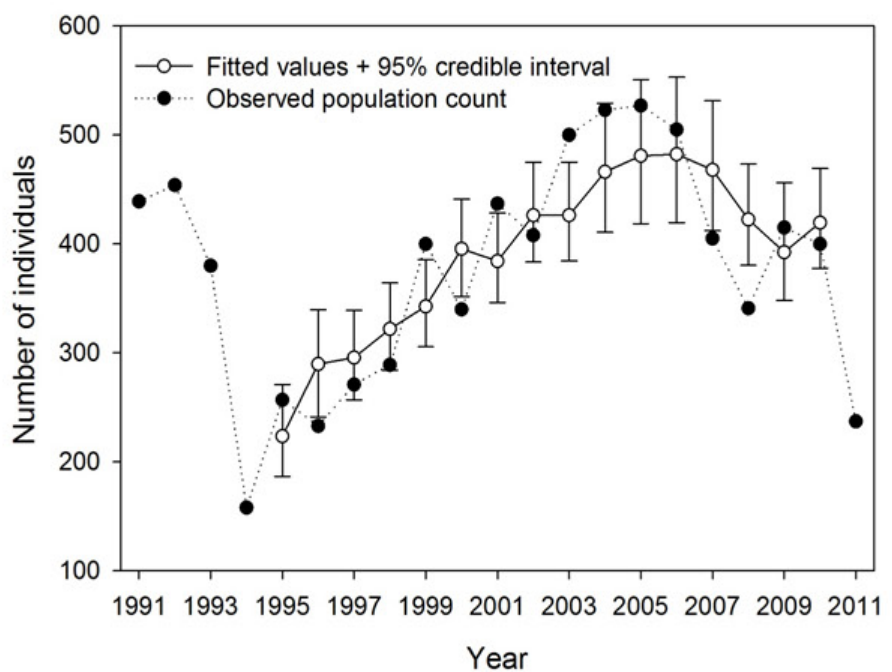

Figure 2.29. Population abundance graphs for five species with long-term monitoring data: (a) Black-footed Albatross, (b) Laysan Albatross, (c) Red-footed Booby, (d) Laysan Teal, and (e) Laysan Finch. Abundance values are an index of population size derived from a subset of the population counted during monitoring surveys. 


\section{Population Dynamics, Carrying Capacity, and Population Vulnerability}

We evaluated avian population vulnerability for the same five sea-level scenarios, assuming that changes in population abundance would be proportional to changes in species-specific nesting habitat. For species showing strong evidence of density dependence, this relationship is better supported. Time series of abundance from long-term monitoring are shown in fig. 2.29, parts (a) through (e). Most species' time series were extremely variable and sampling error could not be estimated.

The observed carrying capacity of Laysan Island to support the breeding bird species with long-term monitoring data ranged from $434 \pm 72$ for Laysan Teal to 284,491 $\pm 43,886$ for Laysan Albatross (table 2.11; carrying capacities expressed as means \pm standard deviations). The only species for which observation error could be measured, the Laysan Teal, showed strong evidence of density dependence (that is, already at carrying capacity; $c=0.68$; table 2.11 ); thus, this species is expected to respond to changes in habitat. It is assumed that species without strong evidence of density dependence ( $c$ greater than 1 ; table 2.11) may respond more gradually or adapt to habitat change, as potential losses in current nesting habitat may be offset by population growth in those areas that are not inundated. In contrast to gradual habitat losses that may reduce the carrying capacity of wildlife populations, additive mortality of chicks and adults in habitats vulnerable to storm impacts may cause more rapid decreases in population abundance.

\section{Discussion}

\section{Systematic Underestimation of Vulnerability from Passive Models}

In comparison to the passive (or "bathtub") inundation models, the dynamic wave-driven inundation models required far lower values of SLR to produce similar magnitudes of inundation as the passive models. We observed that as sea level increased and water depths over the reef crest and reef flats increased, wave-energy attenuation due to wave breaking and bottom friction declined, resulting in more wave energy (larger wave heights and longer wavelengths) reaching the shoreline. Thus as sea level rose in our models, wave-driven water levels rose simultaneously, exacerbating the effects of SLR. This positive feedback cycle suggests that passive models systematically underestimate the amount of inundation that will occur on annual timescales and that the larger the rise in sea level, the greater the degree of underestimation.

\section{Additional Increments of SLR}

Although the passive models considered in this study (SLR less than or equal to $+2.00 \mathrm{~m}$ ) exhibited little inundation on Laysan Island for each SLR scenario, a potential threshold may be reached if an additional $0.50-\mathrm{m}$ increment of SLR $(+2.50 \mathrm{~m})$ were considered. In such a case, inundation would increase dramatically as the low-lying coastal dunes in the south and east quadrants of the island would be overtopped at mean high tide. This scenario would flood the two topographic depressions in the eastern desert and portions of the central basin adjacent to the hyper-saline lake. Under a passive +2.50 -m SLR scenario, the overall inundation pattern would resemble the theoretical maximum amount of flooding predicted by the wave-driven inundation model at the higher SLR scenarios $(+1.50$ and $+2.00 \mathrm{~m})$.

Alternatively, if $+1.50 \mathrm{~m}$ of passive SLR were combined with the mean wave-driven water level during high-surf events $(+1.16 \mathrm{~m}$ at $+1.50 \mathrm{~m}$ of SLR), then potential inundation during high-surf events might equate to a passive rise of $+2.66 \mathrm{~m}$, depending on the volume of wave-driven seawater and infiltration rates. In either case (that is, a passive SLR of $+2.66 \mathrm{~m}$ or a dynamic rise of $+1.50 \mathrm{~m}$ of SLR), the maximum extent of inundation would resemble the documented inundation from the severe February 2011 rainfall and large-wave event (USFWS data; Kristof and others, 2011), as wavedriven seawater would reach and begin to fill the low-lying interior basins. The documented flood event of February 2011 provides an excellent reference level for the predicted inundation extents presented here and provides some validation of the general spatial patterns of our predictions.

As sea level increases, wave-driven inundation becomes more likely to occur in complicated and broad patterns across the island's interior, as noted when comparing the results for the wave-inundation models at +1.50 and $+2.00 \mathrm{~m}$ SLR. Since the volume of seawater along all inundation swaths remains unknown, it is not clear how much seawater will reach the interior basins under either of these two SLR scenarios, only that such a worst-case inundation scenario reflects the flow characteristics of wave-driven seawater and is theoretically possible during large-wave events. Although the likelihood of achieving the maximum theoretical extent of inundation is unclear, as sea level rises and more inundation paths intersect the central lake, greater seawater volumes would reach the lake, increasing the likelihood of achieving the maximum extent of inundation. In the long-term, by the time SLR reaches the height of the southeastern coastal dunes (greater than or equal to $+2.50 \mathrm{~m}$ above MSL), the maximum extent of inundation under the wave-driven modeling approach would become a moot point, as these basins would likely be connected to the ocean at that time. Future modeling that includes potential morphological changes to the island would provide a more accurate assessment of this likelihood.

\section{Chronological Implications of Dynamic Inundation Models}

We investigated the effect of various SLR scenarios without assigning explicit dates to them, although we assumed 
our scenarios represent a range of distinct possibilities over the next century, with $+1.00 \mathrm{~m}$ SLR representing the most likely scenario (given our current knowledge of the state of this system; see Fletcher, 2009). One consequence of considering wave-driven inundation for Laysan Island was that for a given SLR value, wave-driven water levels generally added approximately $1 \mathrm{~m}$ of inundation depth (depending on SLR scenario) to passive inundation surfaces during large-wave events. Thus, since large-wave events have the potential to add water heights comparable to a century's worth of SLR to our passive inundation surfaces, our wave-driven inundation models essentially forecasted comparable levels of inundation nearly a century earlier than passive models. Of course, with such a comparison, the passive model with $+1.00 \mathrm{~m}$ of SLR depicted year-round inundation, while the wave-driven inundation model at present sea level forecasted similar levels of inundation only during the top 5 percent of storm-wave events. As the rate of SLR changes (due to changes in the rate of ice sheet melting, greenhouse gas emissions, etc.), wave-driven inundation will change accordingly as will the patterns and timing of inundation. In spite of these uncertainties over the next century, consideration of wavedriven inundation resulted in significant increases in inundation, with several areas experiencing periodic inundation many decades earlier than forecasted by passive modeling approaches. Uncertainty in the rate of SLR has multiple components including a strong regional component. As noted by Stammer (2010), regional SLR is "most relevant" and "most complex." While mass contributions to the ocean (primarily in the form of melting ice sheets, glaciers, and ice caps) spread rapidly around the world by pressure-related motions (known as barotropic motions), it may take many decades for sea level to respond to both the pressure- and temperature-related forces (in the form of a baroclinic response) from meltwater (Stammer, 2008; Gower, 2010; Church and others, 2011). Despite this potential time lag, recent studies have shown a wide range of regional SLR responses including (1) a high rate of SLR in the Western Pacific corresponding to increased trade winds in the tropical Pacific (Merrifield, 2011), and (2) falling sea level relative to the earth's crust near Greenland and West Antarctica due to decreased weight of the ice sheets, diminished local gravitational forces, and vertical rebounding of the crust (Church and others, 2011). In short, given the complex set of forces operating on regional sea level, a high degree of uncertainty still exists regarding the timing of regional SLR in all areas of the world, including the Central Pacific.

\section{Frequency of Inundation Events}

While flooding from storm-generated waves would occur only during approximately annual storm events and not perennially as it would under a scenario of passive SLR, these periodic injections of seawater into the lake could produce dramatic and permanent changes in lake chemistry and biology, depending upon the frequency of large-wave events. With salinities of 3-4 times that of the ocean and an ecosystem dominated by salt-tolerant species (including algae, Dunaliella spp.; brine shrimp, Artemia franciscana; and brine flies, Scatella sexnotata; Reynolds, 2002), the lake ecosystem is potentially vulnerable to large injections of seawater that could affect lake salinities. Based on interannual variability in climate (for instance, variability between El Niño and La Niña years), we would anticipate the frequency of large-wave events to vary greatly from year to year (USACE, 2011). Likewise, the impacts of these large-wave events would vary annually, depending on event magnitude and the number of events per year. In addition to impacts on Laysan's hyper-saline wetland ecosystem, injections of storm-wave water across the landscape could have significant impacts on surrounding land cover and vegetation, as observed during recent inundation events on Laysan in 2011 including the February storm and March tsunami (USFWS data; Kristof and others, 2011). If mass avian mortality events associated with some severe storms occurred more frequently, dramatic population declines could be expected. Species most at risk to increasing storm frequency and intensity are those attending young chicks during storm events (for instance, Black-footed Albatross, Laysan Albatross, Bonin Petrel, and Tristram's Storm-petrel; see fig. 2.28).

\section{Future Geomorphology}

While the degree of groundwater connectivity between the ocean and lake remains unknown, since no hydrological studies have been conducted for Laysan Island, a comparable rise in groundwater and sea level remains a distinct possibility, especially over the decadal and centurial time periods relevant to climate change and SLR. When we incorporated rising groundwater into the modeling process, the lake area expanded considerably, as did the likelihood of wave-driven seawater reaching the lake zone during large-wave events. Under such a modeling scenario, the island took on a distinct atoll-like appearance (where parts of the island partially enclose a lagoon), providing insight into possible geomorphologic changes in the coming centuries.

While the highest wave-driven water levels generally occurred on the west side of Laysan Island (fig. 2.11), the highest dunes (which exceed $10 \mathrm{~m}$ above MSL) were located on the western side of the island, generally preventing wave-driven seawater from penetrating much beyond the beach. In contrast, the southeast quadrant of the island had much lower coastal dunes (less than $3 \mathrm{~m}$ above MSL), offering the least protection from wave-driven water levels. Thus, in spite of generally lower wave-driven water levels in the southeast, our models indicated that the dunes would be breached here first. Assuming current island morphology, the ocean would be most likely to intersect Laysan's interior lake in the southeast quadrant.

\section{Vulnerability to Outlier Storm Events}

All modeled wave-driven water levels were calculated for the winter season (Dec.-Feb.) only; thus our predicted inundation extents relate to likely levels of inundation as a result of combined SLR and the largest 5 percent of winter 
waves. Based on historical wave data (USACE, 2011), winter on average produces the largest wave heights, longest wave periods, and highest resulting set-up and run-up values of the year. Since storm wave-driven water levels were computed based on the mean of the top 5 percent of winter waves, approximately 2.5 percent of these water levels exceeded calculated mean levels, often by a substantial amount. Consequently some future winter storms are likely to produce wave-driven water levels that greatly exceed the total water levels used in our inundation modeling. Additionally, although mean wave-driven water levels for winter were greater than that of any other season, a single storm event from another season (for example, a summer or fall hurricane) may produce wave-driven water levels in Laysan's vulnerable southeast quadrant that greatly exceed winter levels along this section of coastline. Such an outlier event remains a possibility and could produce extreme wave-driven water levels similar in magnitude to the total water levels (4-9 $\mathrm{m}$ above MLLW) observed on Kaua' $i$ when Hurricane Iniki made landfall (Fletcher and others, 1995). For the island of Laysan, where the lowest coastal dunes lie in the south and east, a category-five hurricane approaching from the southeast represents the worst-case scenario in terms of wave-driven water levels and potential inundation. Since the first official record of a hurricane in Hawaiian waters in 1950, only five category-five hurricanes have occurred in the Central Pacific, with none of these making landfall in the NWHI, although Hurricane Patsy did approach Midway Atoll as a category-three storm (Businger, 2012; Central Pacific Hurricane Center, 2012). More recently (in 2009), Hurricane Neki developed into a category-three hurricane but then weakened into a tropical storm as it crossed the PMNM; although no damage was caused, personnel were evacuated from Laysan Island during this storm (Kristof and others, 2010). Potentially damaging surf events from the south and east may occur, even if a hurricane does not make landfall at Laysan.

\section{Succession of Vegetation}

When analyzing vegetation loss, we evaluated the potential effects of inundation for existing land cover patterns only. However, we know that as the frequency and magnitude of inundation increases, land cover and vegetation patterns will shift accordingly (LaFever and others, 2007). For instance, as sea level rises and inundates existing beach areas, we expect the beach zone and coastal communities to shift upwards with the changing coastline. Likewise for wetland vegetation, a potential rise in lake levels is likely to result in an upward shift in wetland vegetation, rather than a complete disappearance of this land cover class. Simultaneously, other land cover classes may shift upwards as sea level rises, producing a cascade effect, at least for the land cover classes currently at lower elevations. For the interspersed bunch grass and groundcover at the higher elevations (up to $10 \mathrm{~m}$ above MSL), we do not anticipate much change due to SLR in the foreseeable future.

\section{Thresholds of Vulnerability for Nesting Habitat}

Although the severity of impact varied among models and species, less than 10 percent of avian nesting areas were predicted to experience inundation by all models for SLR scenarios less than $+1.00 \mathrm{~m}$; thus below the +1.00 -m threshold, our models predicted limited change across all avian nesting areas and habitats. However, at $+2.00 \mathrm{~m}$ SLR, only the passive inundation model (without wave-driven inundation or groundwater percolation) predicted less than 10 percent direct losses to avian nesting habitats.

The nesting areas for Black-footed Albatross and Brown Booby, which occur around the coastal perimeter of Laysan, were more vulnerable to wave-driven water levels than to rising groundwater. Conversely, the nesting habitat for Red-footed Booby and Great Frigatebird was more vulnerable to rising groundwater and interior lake levels than to wave-driven water levels, as much of their nesting habitat was located near the central lake; because these two species are shrub nesters, we anticipate rising groundwater to limit future shrub distribution rather than to inundate nests directly. Other avian species that were not modeled spatially in this study have similar distribution patterns on Laysan (for instance, along the island perimeter or lake perimeter), and consequently are expected to exhibit similar levels of vulnerability to inundation. For example, Tristram's Storm-petrel, a burrow nester that breeds in a narrow band around the perimeter of the lake (Ely and Clapp, 1973; Marks and Leasure, 1992; McClelland and others, 2008), would be more vulnerable to rising groundwater (that would inundate underground nests) than to wave-driven seawater. Additionally, we anticipate habitat loss to affect the Black Noddy and White Tern, because these species nest in many of the same tree and shrub species as Red-footed Booby and Great Frigatebird.

\section{Implications of SLR for Laysan's Wildlife Populations}

We expect SLR to impact avian populations at Laysan Island by reducing carrying capacity and influencing population dynamics through population limitation and changes in nesting habitat; these factors might lead to smaller population sizes for some species. Although the time scale of SLR is uncertain, we expect the largest population effects from additive mortality due to increasing magnitude and frequency of extreme storm events, rather than from gradual habitat losses from passive inundation scenarios. We expect there to be habitat loss from SLR as well as habitat destruction from periodic high-wave events including permanent physical, chemical, and biological impacts that render some habitats unsuitable. How species adapt to catastrophic events and long-term changes in habitat availability will vary with inter-specific differences in breeding season phenology and life-history characteristics. Some wildlife species demonstrate resilience with the ability to adapt behavior, relocate to alternative 
habitats, and renest after nest loss. Availability of alternative habitat given inter- and intra-species competition on small islands also will influence how species adapt to reductions in habitat (that is, carrying capacity).

Avian populations on Laysan may be adapted to deal with infrequent catastrophes and extreme inundation events, temporarily influencing fecundity and adult survival. During the severe winter storms of 2010 (Nov.-Dec.) and 2011 (Feb.), substantial flooding of low-lying areas inundated more than 20,000 Laysan Albatross and 9,000 Black-footed Albatross nests (17 and 40 percent, respectively), resulting in failed nests and the mortality of adults (USFWS data; Kristof and others, 2011; E. Flint, USFWS, oral commun.). Also in 2011, Laysan Teal produced no offspring, likely a result of severe habitat flooding during the February storm and March tsunami event. As sea levels rise, these storm-driven inundation events may become more frequent and more severe, with higher wavedriven water levels. Increased frequency and severity of such events can have significant population-level impacts on avian species over the long-term (Frederiksen and others, 2008).

Species that are not nesting during seasonal wave-driven inundation events may be able to adjust their nesting distribution to habitat loss. However, some seabird colonies may persist for years without reproductive success. For example, albatross with strong nest-site fidelity have continued to nest at Trig Island in French Frigate Shoals (area 1.5 ha; mean elevation $0.5 \mathrm{~m}$ ) despite frequent overwash events (E. Flint, USFWS, oral commun.). At the other extreme, species such as Sooty Tern that exhibit higher inter-annual variation in colonial nesting area may be less vulnerable to habitat losses and more able to adapt readily to habitat changes.

The effects of habitat reduction could be exacerbated for species such as the Laysan Teal that are at or near carrying capacity because they are the most sensitive to small decreases in habitat abundance (Gaston and others, 2003). A decrease in habitat does not necessarily correspond to a proportional decrease in carrying capacity, as species may be able to adjust their behavior. For nonmigratory endemic land birds that meet all of their life-history requirements at Laysan Island, population dynamics could be affected by nesting habitat losses and changes in local food distribution and abundance (either positive or negative). In contrast, for seabirds that forage at sea and utilize the island for nesting only, the impact of habitat loss on carrying capacity is limited to nesting area abundance. Consequently, the proportional effects of habitat inundation on carrying capacity may differ greatly between seabirds and endangered endemic land birds.

\section{Future Research Directions}

In future iterations of this modeling process, we hope to gain a better understanding of the physical processes that affect wave-driven water levels, including complexities such as hydrodynamic and terrestrial roughness (that impart frictional resistance on waves and run-up, potentially limiting run-up distances), scouring (which may channelize and enable greater run-up distances), and morphodynamic change (changes in beach and sea floor shape due to erosion and/ or deposition). Although our dynamic wave-driven inundation model represents a major advance over passive inundation models, it still has a number of technical limitations that could be addressed with refined treatment of hydrodynamic roughness (distribution of sand patches and coral species), seasonal variation in beach slope, low-latitude beach structure, and infiltration capacity. In order to address these concerns, high-resolution spatial and temporal data (including beach morphology, sedimentology, stratigraphy, and hydrogeology) would be required to better constrain these variables and thus reduce uncertainty in projected wave-driven water levels and inundation extents.

In terms of biological modeling, better avian monitoring data are needed to describe and predict the relationship between climate change and population change over time. Future population monitoring could be improved with independent replicate surveys, especially for the Laysan Finch and Red-footed Booby (Citta and others, 2007). More specifically, replicate counts (either using maximum MIC or other methods; Dearborn and others, 2001; Citta and others, 2007) could reduce sampling variance and better define other parameters (Dennis and others, 2010). The collection of additional species-specific distribution data would allow for detailed analysis of the spatial overlap between nest sites and inundation areas, making for improved species-specific vulnerability assessments to climate change. If resource limitations prevent monitoring of all species, the endangered species and the species we have identified as the most vulnerable to SLR could be targeted first, including those not currently monitored such as Gray-backed Tern, Tristram's Storm-petrel, and Bonin Petrel. These species would serve as good vulnerability indicator species (Citta and others, 2007) because their breeding season overlaps with the period of highest wave energy and their life-history characteristics make them less able to adapt to rapid environmental change.

\section{Conclusions}

This study illustrated substantial differences between passive and dynamic inundation models for Laysan Island and provided the basis for assessing the vulnerability of Laysan's habitat and avifauna to climate change. At the higher SLR scenarios ( $+1.50 \mathrm{~m}$ or greater), passive inundation models severely underestimated the extent of inundation predicted by wave-driven inundation models, as wave-driven water levels added approximately $1 \mathrm{~m}$ of elevation to passive inundation surfaces. Although periodic in nature, wave-driven inundation may cause long-term changes in land cover, vegetation structure and composition, lake chemistry and area, avian habitat, seabird population dynamics, and endangered endemic bird viability. Viewed chronologically, wave-driven inundation models predicted comparable inundation extents many 
decades earlier than forecasted by passive models. At SLR values greater than or equal to $+1.50 \mathrm{~m}$, a threshold was identified, above which coastal dunes were overtopped during periods of high-wave energy and large losses in avian habitat occurred, particularly for species nesting in low-lying interior basins such as Tristram's Storm-petrel, Red-footed Booby, Great Frigatebird, Black Noddy, and White Tern. The nesting habitats of these species were highly vulnerable to large volumes of wave-driven seawater and to rising groundwater. In general, impacts on avian species varied widely, depending on modeling assumptions, nest-site distribution, breeding phenology, and life-history characteristics. Given current trends in SLR, the $+1.50 \mathrm{~m}$ SLR threshold will not occur until the next century, allowing most avifauna decades to adapt to changing sea levels and managers time to plan for natural resource protection.

These conclusions apply specifically to SLR on Laysan Island, as differing topography, bathymetry, morphology, and wildlife on other Pacific islands will result in island-specific impacts, vulnerabilities, and thresholds. Given Laysan's relative size and elevation in comparison to other islands and atolls, we anticipate SLR and wave-driven inundation to cause more extensive impacts on other islands in the Pacific first (see chap. 1). However, given Laysan's unique and diverse ecosystem, the impacts of SLR and wave-driven inundation on Laysan should not be underestimated.

\section{Acknowledgments}

We are grateful to the U.S. Geological Survey (USGS) National Climate Change and Wildlife Science Center, USGS Pacific Coastal and Marine Science Center, USGS Pacific Island Ecosystems Research Center, and the U.S. Fish and Wildlife Service (USFWS) Pacific Islands Refuges Inventory and Monitoring Program for grants funding this research. We also would like to thank the USFWS Hawaiian Islands National Wildlife Refuge, National Oceanic and Atmospheric Administration Pacific Services Center, and Papahānaumokuākea Marine National Monument for their overarching support of this project, the USGS-Deltares co-operative for numerical modeling support. Also, we extend special thanks to L. Fortini (Pacific Islands Climate Change Cooperative), C. Ostrander (Pacific Islands Ocean Observing System), and L. Mehrhoff (USFWS) for reviewing this chapter; M. Stelmach (USFWS) and A. Kristof (USFWS) for mapping assistance; J. Carter (NOAA) for accuracy assessment surveys; J. Hatfield (USGS Patuxent Wildlife Research Center) for statistical support; T. Speetjens (USFWS) for biological data; and S. Nash (Hawai' i Cooperative Studies Unit) for editing assistance. We also thank L. Slack and J. Hendley (USGS, Menlo Park Publishing Service Center) for editing assistance. 


\section{References Cited}

Aiello-Lammens, M.E., Chu-Agor, L., Convertino, M., Fischer, R.A., Linkov, I., and Akcakaya, H.R., 2011, The impact of sea-level rise on Snowy Plovers in Florida-Integrating geomorphological, habitat, and metapopulation models: Global Change Biology, v. 1365, p. 2486-2497.

Amerson, A.B., 1971, The natural history of French Frigate Shoals, Northwestern Hawaiian Islands: Atoll Research Bulletin, v. 150.

Amerson, A.B., Clapp, R.B., and Wirtz, W.O., 1974, The natural history of Pearl and Hermes Reef, Northwestern Hawaiian Islands: Atoll Research Bulletin, v. 174.

Amerson, A.B., and Shelton, P.C., 1976, The natural history of Johnston Atoll, Central Pacific Ocean: Atoll Research Bulletin, v. 192.

Antonelis, G.A., Baker, J.D., Johanos, T.C., Braun, R.C., and Harting, A.L., 2006, Hawaiian monk seal (Monachus schauinslandi): status and conservation issues: Atoll Research Bulletin, v. 543, p. 75-101.

Ashmole, N.P., 1968, Breeding and molt in the white tern (Gygis alba) on Christmas Island, Pacific Ocean: Condor, v. 70 , p. $35-55$.

Athens, J.S., Ward, J.V., and Blinn, D.W., 2007, Vegetation history of Laysan Island, Northwestern Hawaiian Islands: Pacific Science, v. 61, no. 1, p. 17.

Bailey, A.M., 1956, Birds of Midway and Laysan Islands: Denver Museum of Natural History.

Baker, J.D., Littnan, C.L., and Johnston, D.W., 2006, Potential effects of sea level rise on the terrestrial habitat of endangered and endemic megafauna in the Northwestern Hawaiian Islands: Endangered Species Research, v. 4, no. 1, p. $1-10$.

Balazs, G.H., 1976, Green turtle migration in the Hawaiian Archipelago: Biological Conservation, v. 9, p. 125-140.

Balazs, G.H., and Chaloupka, M., 2004, Thirty-year recovery trend in the once depleted Hawaiian green sea turtle stock: Biological Conservation, v. 117, p. 491-498.

Baldwin, P.H., 1945, Fate of the Laysan Rail: Audubon Magazine, v. 47, p. 343-348.

Benning, T.L., LaPointe, D., Atkinson, C.T., and Vitousek, P.M., 2002, Interactions of climate change with biological invasions and land use in the Hawaiian Islands: Modeling the fate of endemic birds using a geographic information system: Proceedings of the Natural Academy of Sciences, v. 99, no. 22, p. 14246-14249.
Bograd, S.J., Foley, D.G., Schwing, F.B., Wilson, C., Laurs, R.M., Polovina, J.J., Howell, E.A., and Brainard, R.E., 2004, On the seasonal and interannual migrations of the transition zone chlorophyll front: Geophysical Research Letters, v. 31.

Booij, N., Ris, R.C., and Holthuijsen, L.H., 1999, A third-generation wave model for coastal regions: model description and validation: Journal of Geophysical Research-Oceans, v. 104, no. C4, p. 7649-7666.

Bowen, B.W., Meylan, A.B., Ross, J.P., Limpus, C.J., Balazs, G.H., and Avise, J.C., 1992, Global population structure and natural history of the green turtle (Chelonia mydas) in terms of matriarchal phylogeny: Evolution, v. 46, no. 4, p. 865-881.

Boyd, A.J., Stelmach, M.W., Soucie, B.C., and Mainardi, J.A., 2009, Trip Report: Laysan Island 24 March 2009 to 19 August 2009: Administrative Report, U.S. Fish and Wildlife Service.

Brodeur, R.D., Mills, C.E., Overland, J.E., Walters, G.E., and Schumacher, J.D., 1999, Evidence for a substantial increase in gelatinous zooplankton in the Bering Sea, with possible links to climate change: Fisheries Oceanography, v. 8, p. 296-306.

Bryan, E.H., 1938, Midway Island, U.S.A.: Paradise of the Pacific, v. 50, no. 6, p. 29-30.

Bryan, E.H., 1942, American Polynesia and the Hawaiian Chain: Honolulu, HI, Tongg Publishing Co.

Bryan, W.A., 1911, Laysan Island-A visit to Hawaii's bird reservation: Mid-Pacific Magazine, v. 4, p. 303-315.

Buddemeier, R.W., and Smith, S.V., 1988, Coral-reef growth in an era of rapidly rising sea-level predictions and suggestions for long term research: Coral Reefs, v. 7, no. 1, p. 51-56.

Businger, S., 2012, Hurricanes in Hawaii, accessed February 8, 2012. at http://www.soest.hawaii.edu/MET/Faculty/businger/poster/hurricane/.

Cazenave, A., and Llovel, W., 2010, Contemporary sea level rise: Annual Review of Marine Science, v. 2, p. 145-173.

Central Pacific Hurricane Center, 2012, Previous tropical storms in the Central Pacific: accessed February 8, 2012. at http://www.prh.noaa.gov/cphc/summaries/.

Chardine, J.W., and Morris, R.D., 1996, Brown noddy (Anous stolidus), in Poole, A., ed., The Birds of North America Online: Ithaca, New York, Cornell Lab of Ornithology.

Childs, C., 2004, Interpolating surfaces in ArcGIS Spatial Analyst: ArcUser, v. Summer 2004, p. 32-34. 
Church, J.A., Gregory, J.M., Huybrechts, P., Kuhn, M., Lambeck, K., Nhuan, M.T., Qin, D., and Woodworth, P.L., 2001, Changes in sea level in climate change 2001: the scientific basis, in Houghton, J.T., Ding, Y., Griggs, D.J., Noguer, M., van der Linden, P., Dai, X., Maskell, K., and Johnson, C.I., eds., Contribution of working group 1 to the third assessment report of the Intergovermental Panel on Climate Change: Cambridge University Press, p. 641-693.

Church, J.A., Gregory, J.M., White, N.J., Platten, S.M., and Mitrovica, J.X., 2011, Understanding and projecting sea level change: Oceanography, v. 24, no. 2, p. 130-143.

Church, J.A., and White, N.J., 2006, A 20th-century acceleration in global sea-level rise: Geophysical Research Letters, v. 33, p. L01602.

Citta, J., Reynolds, M.H., and Seavy, N.E., 2007, Seabird monitoring assessment for Hawaii and the Pacific Islands: University of Hawaii at Hilo, Hawaii Cooperative Studies Unit Technical Report, HSCU-007.

Clague, D.A., 1996, The growth and subsidence of the Hawaiian-Emperor volcanic chain, in Keast, A., and Miller, S.D., eds., The origin and evolution of the Pacific Island biota, New Guinea to Eastern Polynesia: patterns and processes: Amsterdam, The Netherlands, SPB Academic Publishing, p. $35-50$.

Clapp, R.B., and Wirtz, W.O., 1975, The natural history of Lisianski Island, Northwestern Hawaiian Islands: Atoll Research Bulletin, v. 186.

Conant, S., Christensen, C.C., Conant, P., Gagne, W.C., and Goff, M.L., 1984, The unique terrestrial biota of the Northwestern Hawaiian Islands, Proceedings of the symposium on resource investigations in the Northwestern Hawaiian Islands: Honolulu, Hawaii, University of Hawaii Sea Grant College Program, p. 77-94.

Congdon, B.C., Erwin, C.A., Peck, D.R., Baker, G.B., Double, M.C., and O'Neill, P., 2007, Vulnerability of seabirds on the Great Barrier Reef to climate change, in Wachenfeld, D., Johnson, J., Skeat, A., Kenchington, R., Marshall, P., and Innes, J., eds., Climate Change and the Great Barrier Reef: A vulnerability assessment.

Cornett, C., Leopold, D., O’Bryan, J., and Siudzinski, C., 2008, Trip Report: Laysan Island 20 March 2008 to 30 September 2008: Administrative Report, U.S. Fish and Wildlife Service.

Davidson-Arnott, R.G.D., 2005, Conceptual model of the effects of sea level rise on sandy coasts: Journal of Coastal Research, v. 21, no. 6, p. 1166-1172.

Dearborn, D.C., and Anders, A.D., 2006, Demography and reproductive ecology of great frigatebirds: Atoll Research Bulletin, v. 543, p. 159-171.
Dearborn, D.C., Anders, A.D., and Flint, E.N., 2001, Trends in reproductive success of Hawaiian seabirds: is guild membership a good criterion for choosing indicator species?: Biological Conservation, v. 101, p. 97-103.

Deltares (Dutch Institute for Delta Technology), 2011, Delft Hydraulic Software Delft3D.

Dennis, B., and Constantino, R.F., 1988, Analysis of steadystate distributions with the gamma abundance model: application to Tribolium: Ecology, v. 69, p. 1200-1213.

Dennis, B., Ponciano, J.M., Lele, S.R., Taper, M.L., and Staples, D.F., 2006, Estimating density dependence, process noise and observation error: Ecological Monographs, v. 76, p. 323-341.

Dennis, B., Ponciano, J.M., and Taper, M.L., 2010, Replicated sampling increases efficiency in monitoring biological populations: Ecology, v. 91, p. 610-620.

Digital Globe Inc., 2010, Unpublished QuickBird and WorldView-2 satellite imagery: Longmont, Colorado.

Dunlop, J.N., Long, P., Stejskal, I., and Surman, C., 2002, Inter-annual variations in breeding participation at four western Australian colonies of the wedge-tailed shearwater Puffinus pacificus: Marine Ornithology, v. 30, p. 13-18.

Edelstein-Keshat, L., 1988, Mathematical models in biology: New York, McGraw-Hill, 586 p.

Ely, C.A., and Clapp, R.B., 1973, The natural history of Laysan Island, Northwestern Hawaiian Islands: Atoll Research Bulletin, v. 171, p. 1-361.

Engilis, A., and Naughton, M., 2004, U.S. Pacific Islands Regional Shorebird Conservation Plan. U.S. Shorebird Conservation Plan, U.S. Department of the Interior. Portland, Oreg.

ESRI (Environmental Systems Research Institute), 2010, ARCGIS 10 SP3: Redlands, Calif.

Evenhuis, N.L., and Eldredge, L.G., eds., 2004, Natural History of Nihoa and Necker Islands: Honolulu, Hawaii, Bishop Museum Press.

Executive Order 1019, Hawaiian Islands Reservation, February 3, 1909.

Executive Order 13022, Administration of the Midway Islands, October 31, 1996 (61 FR 56875).

Farrell, A., 1928, John Cameron's Odyssey: New York, The Macmillan Publishing Co.

Feare, C.J., 1976, The breeding of the sooty tern (Sterna fuscata) in the Seychelles and the effects of experimental removal of its eggs: Journal of Zoology, v. 179, p. 317-360. 
Fefer, S.I., Harrison, C.S., and Naughton, M.B., 1984, Synopsis of results of recent seabird research in the Northwestern Hawaiian Islands, in Grigg, R.W., and Tanoue, K.Y., Proceedings of the second symposium on resource investigations in the Northwestern Hawaiian Islands: Honolulu, Hawaii, University of Hawaii Sea Grant College Program, p. $9-76$.

Fisher, H.I., 1971, Experiments on homing in Laysan albatross, Diomedea immutabilis: Condor, v. 73, no. 4, p. 389-400.

Fisher, H.I., and Baldwin, P.H., 1946, War and the birds of Midway Atoll: Condor, v. 48, no. 1, p. 3-15.

Fleet, R.R., 1972, Nesting success of the red-tailed tropicbird on Kure Atoll: Auk, v. 89, no. 3, p. 651-659.

Fletcher, C.H., 2009, Sea level by the end of the 21st century: A review: Shore and Beach, v. 77, p. 4-12.

Fletcher, C.H., Richmond, B.M., Barnes, G.M., and Schroeder, T.A., 1995, Marine flooding on the coast of Kaua'i during Hurricane Iniki: Hindcasting inundation components and delineating washover: Journal of Coastal Research, v. 11, no. 1, p. 188-204.

Flint, E., 2011, Albatross population summary: Hawaiian Islands National Wildlife Refuge and Midway Atoll National Wildlife Refuge - annual nest counts through hatch year 2011: Unpublished report for the U.S. Fish and Wildlife Service.

Frederiksen, M., Daunt, F., Harris, M.P., and Wanless, S., 2008, The demographic impact of extreme events: stochastic weather drives survival and population dynamics in a long-lived seabird: Journal of Animal Ecology, v. 77, no. 5, p. 1020-1029.

Friedlander, A.K., Keller, K., Wedding, L., Clarke, A., and Monaco, M., eds., 2009, A marine biogeographic assessment of the Northwestern Hawaiian Islands: NOAA Technical Memorandum NOS NCCOS 84. Prepared by NCCOS's Biogeography Branch in cooperation with the Office of National Marine Sanctuaries Papahānumokuākea Marine National Monument. Silver Spring, Md, 363 p.

Gaston, K.J., Blackburn, T.M., and Glodewijk, K.K., 2003, Habitat conversion and global avian biodiversity loss: Proceedings of the Royal Society, v. 270, p. 1293-1300.

Gauger, V.H., 1999, Black noddy (Anous minutus), in Poole, A., ed., The Birds of North America Online: Ithaca, New York, Cornell Lab of Ornithology.
Gesch, D.B., Guiterezz, B.T., and Gill, S.K., 2009, Coastal Elevations, Coastal sensitivity to sea level rise: A focus on the Mid-Atlantic Region. A report by the U.S. Climate Change Science Program, and the Subcommittee on Global Change Research: Washington D.C., Government Printing Office, p. 25-42.

Gingerich, S.B., 2008, Ground-water availability in the Wailuku area, Maui, Hawaii: U.S. Geological Survey Scientific Investigations Report 2008-5236, p. 95.

Gower, J.F.R., 2010, Comment on: Response of the global ocean to Greenland and Antarctic ice melting by D. Stammer: Journal of Geophysical Research, v. 115, no. C10009.

Grace, J., and Anderson, D.J., 2009, Masked booby (Sula dactylatra), in Poole, A., ed., The Birds of North America Online: Ithaca, New York, Cornell Lab of Ornithology.

Grant, G.S., Warham, J., Pettit, T.N., and Whittow, G.C., 1983, Reproductive behavior and vocalizations of the Bonin petrel: Wilson Bulletin, v. 95, no. 4, p. 522-539.

Grebmeier, J.M., Overland, J.E., Moore, S.E., Farley, E.V., Carmack, E.C., Cooper, L.W., Frey, K.E., Helle, J.H., McLaughlin, F.A., and McNutt, S.L., 2006, A major ecosystem shift in the northern Bering Sea: Science, v. 311, p. 1461-1464.

Grigg, R.W., 1998, Holocene coral reef accretion in Hawaii: a function of wave exposure and sea level history: Coral Reefs, v. 17, p. 263-272.

Grigg, R.W., 2008, The Darwin Point: a conceptual and historical view, in Proceedings of the 11th International Coral Reef Symposium, Ft. Lauderdale, Fla.

Grinsted, A., Moore, J.C., and Jevrejeva, S., 2010, Reconstructing sea level from paleo and projected temperatures 200 to 2100 AD: Climate Dynamics, v. 34 , no. 4, p. 461-472.

Hammond, R.L., Stelmach, M.W., Granberg, R.M., and Taylor, W.M., 2010, Trip report-Laysan Island 18 March 2010 to 8 August 2010: Administrative Report, U.S. Fish and Wildlife Service.

Hatfield, J., Reynolds, M.H., Seavy, N.E., and Krause, C.M., 2012, Population dynamics of Hawaiian seabird colonies vulnerable to sea-level rise: Conservation Biology, v. 26, no. 4, p. 667-678.

Hawaiian Ecosystems at Risk Project, 2010, PIER species info: Pluchea indica. www.hear.org, v. 2011.

Hijmans, R.J., Cameron, S.E., Parra, J.L., Jones, P.G., and Jarvis, A., 2005, Very high resolution interpolated climate surfaces for global land areas: International Journal of Climatology, v. 25, p. 1965-1978. 
Hoeke, R., Storlazzi, C., and Ridd, P., 2011, Hydrodynamics of a bathymetrically complex fringing coral reef embayment: wave climate, in situ observations, and wave prediction: Journal of Geophysical Research-Oceans, v. 116.

Holthuijsen, L.H., Booij, N., and Ris, R.C., 1993, A spectral wave model for the coastal zone, in 2nd International Symposium on Ocean Wave Measurement and Analysis, New Orleans, p. 630-641.

IPCC (Intergovernmental Panel on Climate Change), 2007, Climate Change 2007: The physical science basis. Contribution of Working Group I to the Fourth Assessment Report of the Intergovernmental Panel on Climate Change: Cambridge University Press, 996 p.

Jevrejeva, S., Moore, J.C., Grinsted, A., and Woodworth, P.L., 2008, Recent global sea level acceleration started over 200 years ago?: Geophysical Research Letters, v. 35, no. L08715, p. 1-4.

Jokiel, P.L., and Brown, E.K., 2004, Global warming, regional trends and inshore environmental conditions influence coral bleaching in Hawaii: Global Change Biology, v. 10, p. $1627-1641$.

Juvik, J.O., Rodomsky, B.T., Price, J.P., Hansen, E.W., and Kueffer, C., 2011, The upper limits of vegetation on Mauna Loa, Hawaii: a 50th anniversary assessment: Ecology, v. 92, no. 2, p. 518-525.

Kepler, C.B., 1967, Polynesian rat predation on nesting Laysan albatrosses and other Pacific seabirds: Auk, v. 84, no. 3, p. 426-430.

Kikiloi, K., 2010, Rebirth of an archipelago: Sustaining a Hawaiian cultural identity for people and homeland: Hulili: Multidisciplinary research on Hawaiian well-being, v. 6, p. 73-115.

Klavitter, J., 2006, Native plants of Midway Atoll NWR: U.S. Fish and Wildlife Service.

Kristof, A.A., Stelmach, M.W., Soucie, B.C., Larish, M., Fox, A., Waddington, C., and Waddington, J., 2010, Trip Report: Laysan Island 11 August 2009 to 22 March 2010: Administrative Report, U.S. Fish and Wildlife Service.

Kristof, A.A., Watson, J.C., Cook, E.L., and Tyhurst, P.C., 2011, Trip Report: Laysan Island 8 August 2010 to 30 March 2011: Administrative Report, U.S. Fish and Wildlife Service.

LaFever, D.H., Lopez, R.R., Feagin, R.A., and Silvy, N.J., 2007, Predicting the impacts of future sea-level rise on an endangered lagomorph: Environmental Management, v. 40, p. $430-437$.
Laidre, K.L., Stirling, I., Lowry, L.F., Wiig, Ø., Heide-Jørgensen, M.P., and Ferguson, S.H., 2008, Quantifying the sensitivity of Arctic marine mammals to climate-induced habitat change: Ecological Applications, v. 18, no. 2 Supplement, p. 97-125.

Laniawe, L., 2004, Midway Atoll National Wildlife Refuge: GIS maps of Sand, Eastern, and Spit Islands: Report for the U.S. Fish and Wildlife Service.

LaPointe, D., Goff, M.L., and Atkinson, C.T., 2010, Thermal constraints to the sporogonic development and altitudinal distribution of avian malaria Plasmodium relictum in Hawaii: Journal of Parasitology, v. 96, no. 2, p. 318-324.

Lawler, J.J., Shafer, S.L., White, D., Kareiva, P., Maurer, E.P., Blaustein, A.R., and Bartlein, P.J., 2009, Projected climateinduced faunal change in the Western Hemisphere: Ecology, v. 90 , no. 3 , p. 588-597.

Leuliette, E.W., 2012, Sea level trend map: National Oceanic and Atmospheric Administration Laboratory for Satellite Altimetry sea level rise products, accessed July 31, 2012, at http://ibis.grdl.noaa.gov/SAT/SeaLevelRise/LSA_SLR_ maps.php.

Li, X., Rowley, R.J., Kostelnick, J.C., Braaten, D., Meisel, J., and Hulbutta, K., 2009, GIS analysis of global impacts from sea level rise: Photogrammetric Engineering and Remote Sensing, v. 75, no. 7, p. 807-818.

Link, W.A., and Barker, R.J., 2010, Bayesian inference with ecological applications: New York, Academic Press.

Loarie, S.R., Duffy, R.B., Hamilton, H., Asner, G.P., Field, C.B., and Ackerly, D.D., 2009, The velocity of climate change: Nature, v. 462, p. 1052-1057.

Lowe, R.J., Falter, J.L., Bandet, M.D., Pawlak, G., Atkinson, M.J., Monismith, S.G., and Koseff, J.R., 2005, Spectral wave dissipation over a barrier reef: Journal of Geophysical Research-Oceans, v. 110, no. C4.

Lowe, R.J., Falter, J.L., Monismith, S.G., and Atkinson, M.J., 2009, A numerical study of circulation in a coastal reeflagoon system: Journal of Geophysical Research-Oceans, v. 114.

Lunn, D.J., Thomas, A., Best, N., and Speigelhalter, D., 2000, WinBUGS - a Bayesian modeling framework: concepts, structure, and extensibility: Statistics and Consulting, v. 10, p. 325-337.

Marks, J.S., and Leasure, S.M., 1992, Breeding biology of Tristram's storm-petrel on Laysan Island: The Wilson Bulletin, v. 104, no. 4, p. 719-731. 
Marshall, A.P., 1991, Censusing Laysan ducks Anas laysanensis: a lesson in the pitfalls of estimating threatened species populations: Bird Conservation International, v. 2, p. 239-251.

Masters, G., and Norgrove, L., 2010, Climate change and invasive alien species: Commonwealth Agriculture Bureaux International Working Paper 1, accessed August 7, 2012, at http://www.cabi.org/Uploads/File/CABi\%20worldwide/ Invasive \%20alien\%20species\%20working\%20paper.pdf.

McClelland, G.T.W., Jones, I.L., Lavers, J.L., and Sato, F., 2008, Breeding biology of Tristram's storm-petrel Oceanodroma tristrami at French Frigate Shoals and Laysan Island, Northwestern Hawaiian Islands: Marine Ornithology, v. 36, p. $175-181$.

McClung, A., 2005, A population viability analysis of the Laysan finch (Telespiza cantans): Honolulu, University of Hawai'i, Ph.D. dissertation.

McDermond, D.K., and Morgan, K.H., 1993, Status and conservation of North Pacific albatrosses, in Vermeer, K., Briggs, K.T., Morgan, K.H., and Siegel-Causey, D., eds., The status, ecology and conservation of marine birds of the North Pacific: Special Publication Canadian Wildlife Service, p. 70-81.

Megyesi, J.L., and Griffin, C.R., 1996, Breeding biology of the Brown noddy on Tern Island, Hawaii: The Wilson Bulletin, v. 108 , no. 2, p. 317-334.

Megyesi, J.L., and O'Daniel, D.L., 1997, Bulwer's petrel (Bulweria bulwerii), in Poole, A., ed., The Birds of North America Online: Ithaca, New York, Cornell Lab of Ornithology.

Menon, S., Soberon, J., Li, X., and Peterson, A.T., 2010, Preliminary global assessment of terrestrial biodiversity consequences of sea-level rise mediated by climate change: Biodiversity Conservation, v. 19, p. 1599-1609.

Merrick, 2011, MARS Merrick Advanced Remote Sensing Software (7.0 ed.): Aurora, Colo.

Merrifield, M.A., 2011, A shift in Western Tropical Pacific sea level trends during the 1990s: Journal of Climate, v. 24, p. 4126-4138.

Merrifield, M.A., Merrifield, S.T., and Mitchum, G.T., 2009, An anomalous recent acceleration of global sea level rise: Journal of Climate, v. 22, p. 5772-5781.

Metz, V.G., and Schreiber, E.A., 2002, Great frigatebird (Fregata minor), in Poole, A., ed., The Birds of North America Online: Ithaca, New York, Cornell Lab of Ornithology.

Mitchell, G., and MacNabb, K., 2010, High resolution stereo satellite elevation mapping accuracy assessment, ASPRS 2010 Annual Conference: San Diego, Calif.
Montaggioni, L.F., 2005, History of Indo-Pacific coral reef systems since the last glaciation: development patterns and controlling factors: Earth-Science Reviews, v. 71, no. 1-2, p. $1-75$.

Montevecchi, W.A., and Myers, R.A., 1997, Centurial and decadal oceanographic influences on changes in northern gannet populations and diets in the northwest Atlantic: implications for climate change: ICES: Journal of Marine Science, v. 54, p. 608-614.

Moore, J., 2009, A comparative analysis of population estimation methods for a burrow-nesting seabird: A novel groundcount method and closed population capture-recapture modeling Halifax, Nova Scotia, Saint Mary's University, M.S. thesis, $125 \mathrm{p}$.

Morin, M., 1991, The breeding ecology of the Laysan finch (Drepanidinae telespiza cantans) on Laysan Island: Honolulu, University of Hawaii Manoa, Ph.D. dissertation.

Morin, M.P., 1992a, The breeding biology of an endangered Hawaiian honeycreeper, the Laysan finch: Condor, v. 94, p. 646-667.

Morin, M.P., 1992b, Laysan finch nest characteristics, nest spacing, and reproductive success in two vegetation types: Condor, v. 94, p. 344-357.

Morin, M.P., and Conant, S., 2002, Laysan finch (Telespiza cantans) and Nihoa finch (Telespiza ultima), in Poole, A., and Gill, F., eds., The Birds of North America Online: Ithaca, New York, Cornell Lab of Ornithology.

Morin, M.P., Conant, S., and Conant, P., 1997, Laysan and Nihoa millerbird (Acrocephalus familiaris), in Poole, A., ed., The Birds of North America Online: Ithaca, Cornell Lab of Ornithology.

Mostello, C.S., Palaia, N.A., and Clapp, R.B., 2000, Graybacked tern (Onychoprion lunatus), in Poole, A., ed., The Birds of North America Online: Ithaca, New York, Cornell Lab of Ornithology.

Moulton, D.W., and Marshall, A.P., 1996, Laysan duck (Anas laysanensis), in Poole, A., ed., The Birds of North America Online: Ithaca, New York, Cornell Lab of Ornithology.

Mousavi, M.E., Irish, J.L., Frey, A.E., Olivera, F., and Edge, B.L., 2011, Global warming and hurricanes: the potential impact of hurricane intensification and sea level rise on coastal flooding: Climate Change, v. 104, p. 575-597.

National Hurricane Center, 2011, Hurricane Preparedness: Slosh Model. http://www.nhc.noaa.gov/HAW2/english/ surge/slosh.shtml

National Wildlife Refuge System Administration Act, 1966, as amended 16 U.S.C. $§ 106-580$, et seq. 
Naughton, M., 1982, Breeding biology of the Christmas shearwater (Puffinus nativitatus) on Laysan Island, Hawaii: Pacific Seabird Group Bulletin, v. 9, no. 2, p. 71-72.

Nelson, J.B., 1978, The Sulidae: Oxford, United Kingdom, Oxford University Press.

Nicholls, R.J., and Cazenave, A., 2010, Sea-level rise and its impact on coastal zones: Science, v. 328, no. 5985, p. $1517-1520$.

Niethammer, K.R., Balazs, G.H., Hatfield, J.S., Nakai, L., and Megyesi, J.L., 1997, Reproductive biology of the green turtle (Chelonia mydas) at Tern Island, French Frigate Shoals, Hawaii: Pacific Science, v. 51, p. 36-47.

Niethammer, K.R., and Patrick, L.B., 1998, White tern (Gygis alba), in Poole, A., ed., The Birds of North America Online: Ithaca, New York, Cornell Lab of Ornithology.

NOAA (National Oceanic and Atmospheric Administration), 1986, Designated critical habitat; Hawaiian monk seal: Federal Register, v. 51.

NOAA (National Oceanic and Atmospheric Administration), 1988, Critical habitat; Hawaiian monk seal; Endangered Species Act: Federal Register, v. 53, no. 102.

NOAA (National Oceanic and Atmospheric Administration), 2007, Recovery plan for the Hawaiian monk seal (Monachus schauinslandi).

NOAA (National Oceanic and Atmospheric Administration), 2009, Coastal inundation mapping guidebook. NOAA Coastal Services Center: Charleston, S.C.

NOAA (National Oceanic and Atmospheric Administration), 2010a, Mapping Inundation Uncertainty: NOAA Coastal Services Center, Charleston, S.C.

NOAA (National Oceanic and Atmospheric Administration), 2010b, Technical consideration for use of geospatial data in sea level change mapping and assessment. NOAA Technical Report NOS 2010-01: NOAA National Ocean Service Center for Operational Oceanographic Products and Services, $130 \mathrm{p}$.

NOAA (National Oceanic and Atmospheric Administration), 2011a, Northwestern Hawaiian Islands Lidar validation mission: Honolulu, HI, NOAA Pacific Services Center.

NOAA (National Oceanic and Atmospheric Administration), 2011b, Tides and Currents: Silver Spring, Md, COOPS (Center for Operational Oceanographic Products and Services).

NOAA (National Oceanic and Atmospheric Administration) Office of Coast Survey, 2011, Nautical Charts and Pubs. (Also available at http:/www.nauticalcharts.noaa.gov/mcd/ Raster/)
Olson, S.L., 1996, History and ornithological journals of the Tanager expedition of 1923 to the Northwestern Hawaiian Islands, Johnston and Wake Islands: Atoll Research Bulletin, v. 433, p. 1-210.

Olson, S.L., and Ziegler, A.C., 1995, Remains of land birds from Lisianski Island, with observations on the terrestrial avifauna of the Northwestern Hawaiian Islands: Pacific Science, v. 49, p. 111-125.

Overpeck, J.T., Otto-Bliesner, B.L., Miller, G.H., Muhs, D.R., Alley, R.B., and Kiehl, J.T., 2006, Paleoclimatic evidence for future ice-sheet instability and rapid sea level rise: Science, v. 311, no. 1747-1750.

Pacific Islands Benthic Habitat Mapping Center, 2011, Bathymetric gridded data, Northwest Hawaiian Islands: Laysan Island, accessed June 15, 2011, at http://www.soest.hawaii. edu/pibhmc/pibhmc_nwhi_lay_bathy.htm.

Papahānumokuākea Marine National Monument, 2008, Papahānumokuākea Marine National Monument management plan: Honolulu, Hawaii, p. 372 p.

Parmesan, C., 2006, Ecological and evolutionary responses to recent climate change: Annual Review of Ecology, Evolution, and Systematics, v. 37, p. 637-669.

Pfeffer, W.T., Harper, J.T., and O’Neel, S., 2008, Kinematic constraints on glacier contributions to 21 st-century sea-level rise: Science, v. 321, no. 5894, p. 1340-1343.

PhotoSat Information Ltd., 2010, Digital Terrain Models: Vancouver, British Columbia.

PMNM (Papahānumokuākea Marine National Monument), 2008, Papahānumokuākea Marine National Monument management plan: Honolulu, Hawaii, p. 372 p.

Polovina, J.J., Howell, E., Kobayashi, D.R., and Seki, M.P., 2001, The transition zone chlorophyll front, a dynamic global feature defining migration and forage habitat for marine resources: Progress in Oceanography, v. 49, p. 1-4.

Poole, A., ed., The Birds of North America Online: http://bna. birds.cornell.edu/BNA/. Ithaca, New York.

Presidential Proclamation 8031, 15 June 2006, Establishment of the Northwestern Hawaiian Islands Marine National Monument (71 FR 36443).

Pyle, P., Welch, A.J., and Fleischer, R.C., 2011, A new species of shearwater (Puffinus) recorded from Midway Atoll, Northwestern Hawaiian Islands: Condor, v. 113, no. 3, p. $518-527$.

Pyle, R.L., and Pyle, P., 2009, The birds of the Hawaiian Islands: occurrence, history, distribution, and status, B.P. Bishop Museum, Honolulu, Hawaii, Version 1. (Also available at http://hbs.bishopmuseum.org/birds/rlp-monograph). 
Rahmstorf, S., 2007, A semi-empirical approach to projecting future sea-level rise: Science, v. 315, p. 368-370.

Rahmstorf, S., 2010, A new view on sea level rise: Nature, v. 4, p. 44-45.

Rauzon, M.J., 2001, Isles of Refuge: Wildlife and History of the Northwestern Hawaiian Islands: Honolulu, Hawaii, University of Hawaii Press.

Rauzon, M.J., and Kenyon, K.W., 1984, White tern nest sites in altered habitat: 'Elepaio, v. 44, no. 79-80.

Reynolds, M.H., 2002, The foraging ecology, population dynamics, and habitat use of Laysan teal (Anas laysanensis): Blacksburg, Va., Virginia Polytechnic and State University, Ph.D. dissertation.

Reynolds, M.H., 2004, Habitat use and home range of the Laysan teal on Laysan Island, Hawaii: Waterbirds, v. 27, no. 2, p. 183-192.

Reynolds, M.H., and Citta, J.J., 2007, Post-fledging survival of Laysan ducks: Journal of Wildlife Management, v. 71, no. 383-388.

Reynolds, M.H., Crampton, L.H., and Vekasy, M.S., 2007, Laysan teal Anas laysanensis nesting phenology and site characteristics on Laysan Island: Wildfowl, v. 57, p. 54-67.

Reynolds, M.H., Hatfield, J.S., Crampton, L.H., Vekasy, M.S., and Tweed, E., 2010, Circadian habitat use, home range and behaviour of Laysan teal Anas Laysanensis: Wildlfowl, v. 60, p. 106-123.

Rice, D.W., and Kenyon, K.W., 1962, Breeding distribution, history, and populations of North Pacific albatrosses: Auk, v. 79 , no. 3 , p. $365-386$.

Richardson, K., Steffen, W., Schellnhuber, H.J., Alcamo, J., Barker, T., Kammen, D.M., Leemans, R., Liverman, D., Munasinghe, M., Osman-Elasha, B., and Waever, O., 2009, Climate Change Global Risks, Challenges and Decisions, Synthesis Report: University of Copenhagen, 39 p.

Ris, R.C., Holthuijsen, L.H., and Booij, N., 1999, A thirdgeneration wave model for coastal regions - 2. Verification: Journal of Geophysical Research-Oceans, v. 104, no. C4, p. 7667-7681.

Robbins, C.S., 1966, Birds and aircraft on Midway Islands 1959-1963 investigations: Special Scientific Report: Wildlife No. 85, U.S. Fish and Wildlife Service, Washington, D.C.

Rotzoll, K., El-Kadi, A.I., and Gingerich, S.B., 2007, Estimating hydraulic properties of volcanic aquifers using constantrate and variable-rate aquifer tests: Journal of the American Water Resources Association, v. 43, no. 2, p. 334-345.
Saliva, J.E., and Burger, J., 1989, Effect of experimental manipulation of vegetation density on nest-site selection in sooty terns: Condor, v. 91, p. 689-698.

Schreiber, B.A., and Schreiber, R.W., 2009, Red-tailed tropicbird (Phaethon rubricauda) in Poole, A., ed., The Birds of North America Online: Ithaca, New York, Cornell Lab of Ornithology.

Schreiber, E.A., Feare, C.J., Harrington, B.A., Murray, B.G., Robertson, W.B., Robertson, M.J., and Woolfenden, G.E., 2002, Sooty tern (Onychoprion fuscatus), in Poole, A., ed., The Birds of North America Online: Ithaca, New York, Cornell Lab of Ornithology.

Schreiber, E.A., and Norton, R.L., 2002, Brown booby (Sula leucogaster), in Poole, A., ed., The Birds of North America Online: Ithaca, New York, Cornell Lab of Ornithology.

Schreiber, E.A., Schreiber, R.W., and Schenk, G.A., 1996, Red-footed booby (Sula sula), in Poole, A., ed., The Birds of North America Online: Ithaca, New York, Cornell Lab of Ornithology.

Schreiber, R.W., and Schreiber, B.A., 1984, Central Pacific seabirds and the El Niño Southern Oscillation: 1982 to 1983 perspectives: Science, v. 225, p. 713-716.

Seavy, N.E., and Reynolds, M.H., 2009, Seabird nest counts: a test of monitoring metrics using red-tailed tropicbirds: Journal of Field Ornithology, v. 80, no. 3, p. 297-302.

Seavy, N.E., Reynolds, M.H., Link, W.A., and Hatfield, J.S., 2009, Postcatastrophe population dynamics and density dependence of an endemic island duck: Journal of Wildlife Management, v. 73, no. 3, p. 414-418.

Seto, N.W., 2001, Christmas shearwater (Puffinus nativitatis), in Poole, A., ed., The Birds of North America Online: Ithaca, New York, Cornell Lab of Ornithology.

Slotterback, J.W., 2002, Tristram's storm-petrel (Oceanodroma tristrami), in Poole, A., ed., The Birds of North America Online: Ithaca, New York, Cornell Lab of Ornithology.

Smithers, B.V., Peck, D.R., Krockenberger, A.K., and Congdon, B.C., 2004, Elevated sea-surface temperature, reduced provisioning and reproductive failure of wedge-tailed shearwaters (Puffinus pacificus) in the southern Great Barrier Reef, Australia: Marine and Freshwater Research v. 54, no. 8, p. 973-977.

Speulda-Drews, L.A., 2010, Midway Atoll National Wildlife Refuge Historic Preservation Plan 2010: U.S. Fish and Wildlife Service, Midway Atoll National Wildlife Refuge, Honolulu, Hawaii, p. 102. 
Sprague, J.C., 2003, Alien species control plan and biological assessment at Pearl and Hermes Reef, Northwestern Hawaiian Islands, 19 May to 4 August, 2003: Administrative Report, U.S. Fish and Wildlife Service.

Stammer, D., 2008, Response of the global ocean to Greenland and Antarctic ice melting: Journal of Geophysical Research, v. 13 , no. C06022.

Stammer, D., 2010, Reply to comment by J.F.R. Gower on "Response of the global ocean to Greenland and Antarctic ice melting" Journal of Geophysical Research, v. 115, no. C10010.

Staples, D.F., Taper, M.L., and Dennis, B., 2004, Estimating population trend and process variation for PVA in the presence of sampling error: Ecology, v. 85, p. 923-929.

Staples, D.F., Taper, M.L., and Shepard, B.B., 2005, Riskbased viable population monitoring: Conservation Biology, v. 19, p. 1908-1916.

Starr, F., and Martz, K., 1999a, S. S. Midway ExpeditionTrip Report: U.S. Fish and Wildlife Service, 35 p.

Starr, F., and Martz, K., 1999b, Trip report, S.S. Midway Expedition, May 21 1999-June 16 1999: Unpublished report for the U.S. Fish and Wildlife Service.

Starr, F., Martz, K., and Loope, L., 2001, Botanical inventory of Kure Atoll: Prepared for Department of Land and Natural Resources, Division of Forestry and Wildlife, Honolulu, Hawaii.

Starr, F., Starr, K., and Loope, L., 2006, Annotated checklist of the vascular plants on Midway Atoll, Hawaii: An addendum to the 1999 botanical survey of Midway Atoll.

Starr, F., Starr, K., and Loope, L., 2008, Botanical Survey of Midway Atoll: Prepared for the United States Fish and Wildlife Service, p. 242.

Stockdon, H.F., Holman, R.A., Howd, P.A., and Sallenger, A.H., 2006, Empirical parameterization of setup, swash, and runup: Coastal Engineering, v. 53, no. 7, p. 573-588.

Storlazzi, C.D., and Reid, J.A., 2010, The influence of El Nino-Southern Oscillation (ENSO) cycles on wave-driven sea-floor sediment mobility along the central California continental margin: Continental Shelf Research, v. 30, no. 14, p. $1582-1599$.

Storlazzi, C.D., and Wingfield, D.K., 2005, The spatial and temporal variability in oceanographic and meteorologic forcing along central California: 1980-2002: U.S. Geological Survey Scientific Investigations Report 2005-5085, 45 p., available at http://pubs.usgs.gov/sir/2005/5085/.
Tabor, K., and Williams, J.W., 2010, Globally downscaled climate projections for assessing the conservation impacts of climate change: Ecological Applications, v. 20, no. 2, p. 554-565.

Timm, O., and Diaz, H.F., 2009, Synoptic-statistical approach to regional downscaling of IPCC twenty-first century climate projections: seasonal rainfall over the Hawaiian Islands: Journal of Climate, v. 22, p. 4261-4280.

Tiwari, M., Balazs, G.H., and Hargrove, S., 2010, Estimating carrying capacity at the green turtle nesting beach of East Island, French Frigate Shoals: Marine Ecology Progress Series, v. 419, p. 289-294.

UNESCO (United Nations Educational Scientific and Cultural Organization), 2010, Report of the decisions adopted by the World Heritage Committee at its 34th session.

USACE (U.S. Army Corps of Engineers), 2011, Wave Information Studies, Wave Hindcast Model Domains for U.S. Coasts, accessed June 15, 2011. at http://frf.usace.army.mil/ wis2010/wis.shtml

USFWS (U.S. Fish and Wildlife Service), 1998, Final Recovery Plan for Three Plant Species on Nihoa Island, Portland, Oregon.

USFWS (U.S. Fish and Wildlife Service), 2003, Endangered and threatened wildlife and plants; designation of critical habitat for five plant species from the Northwestern Hawaiian Islands, Hawaii: Federal Register, v. 68, no. 99.

USFWS (U.S. Fish and Wildlife Service), 2005, Regional seabird conservation plan, Pacific Region: Portland, Oreg., Migratory Birds and Habitat Programs, Pacific Region.

USFWS (U.S. Fish and Wildlife Service), 2007, 5-Year Review of Amaranthus brownii: Honolulu, HI.

USFWS (U.S. Fish and Wildlife Service), 2008a, 5-Year Review of Mariscus pennatiformis: Honolulu, HI.

USFWS (U.S. Fish and Wildlife Service), 2008b, Birds of conservation concern: Arlington, Va., Division of Migratory Bird Management.

USFWS (U.S. Fish and Wildlife Service), 2010, 5-Year Review Summary and Evaluation of Sesbania tomentosa: Honolulu, Hawaii.

USFWS (U.S. Fish and Wildlife Service), 2011a, Rare shorttailed albatross chick banded at Midway Atoll National Wildlife Refuge, Hawaiian and Pacific Islands National Wildlife Refuge Complex News Release: Honolulu, Hawaii. (Also available at: http://www.fws.gov/pacific/news/news. cfm?id=2144374802). 
USFWS (U.S. Fish and Wildlife Service), 2011b, Release of Nihoa millerbirds on Laysan Island offers new hope for critically endangered species, Hawaiian and Pacific Islands National Wildlife Refuge Complex News Release: Honolulu, Hawaii. (Also available at: http://www.fws.gov/ pacific/news/index.cfm).

USFWS (U.S. Fish and Wildlife Service), 2011c, Seabird losses at Midway Atoll National Wildlife Refuge greatly exceed early estimates, Hawaiian and Pacific Islands National Wildlife Refuge Complex News Release: Honolulu, Hawaii. (Also available at: http://www.fws.gov/midway/TsunamiSeabirdLossesFinal031811.pdf).

USGS (U.S. Geological Survey), 2010, U.S. Geological Survey, National Geospatial Program, Lidar Guidelines and Base Specification, p. 18 p., accessed May 27, 2012, at http://lidar.cr.usgs.gov/USGS-NGP\%2020Lidar\%20 20Guidelines\%2020and\%2020Base\%2020Specification\%2020v2013(ILMF).pdf.

Vadenbosch, R., 2000, Effects of ENSO and PDO events on seabird populations as revealed by Christmas bird count data: Waterbirds, v. 23, no. 2, p. 416-422.

Vanderlip, C., Eijzenga, J., Eijzenga, H., McGuire-Ryder, C., Ryder, M., Smith, R., Plentovich, S., Marie, A., and Hester, M., 2007, Kure Atoll Field Report 2007: Honolulu, Hawaii, State of Hawaii, Department of Land and Natural Resources, Division of Forestry and Wildlife.

Vanderlip, C., Marie, A., Hester, M., and Metzler, K., 2008, Kure Atoll Field Report 2008: Honolulu, Hawaii, State of Hawaii, Department of Land and Natural Resources, Division of Forestry and Wildlife.

Vermeer, M., and Rahmstorf, S., 2009, Global sea level linked to global temperature: Proceedings of the National Academy of Sciences, v. 106, no. 51, p. 21527-21532.

Wagner, W.L., Herbst, D.R., and Lorence, D.H., 2005, Flora of the Hawaiian Islands website. (Also available at: http:// botany.si.edu/pacificislandbiodiversity/hawaiianflora/index. htm), v. 2012, no. March.

Walton Jr., T.L., and Ahrens, J., 1989, Maximum periodic runup on smooth slopes: Journal of Waterway, Port, and Ocean Engineering, v. 115, p. 703-708.
Ward, R.G., 1967, American Activities in the Central Pacific 1790-1840: Ridgewood, N.J., Gregg Press, v. 4, 695 p.

Warham, J., 1996, The behavior, population biology and physiology of the petrels: San Diego, Calif., Academic Press.

Webb, A.P., and Kench, P.S., 2010, The dynamic response of reef islands to sea-level rise: evidence from multi-decadal analysis of island change in the Central Pacific: Global and Planetary Change, v. 72, p. 234-246.

Wegmann, A., and Kropidlowski, S., 2002, Alien species control plan and biological assessment at Pearl and Hermes Reef, Northwestern Hawaiian Islands, 24 March to 15 May, 2002: Administrative Report, U.S. Fish and Wildlife Service.

Wetmore, A., 1925, Bird life among lava rock and coral sand: National Geographic Magazine, v. 48, p. 76-108.

Whittow, G.C., 1997, Wedge-tailed shearwater (Puffinus pacificus), in Poole, A., ed., The Birds of North America Online: Ithaca, New York, Cornell Lab of Ornithology.

Woodward, P.W., 1972, The natural history of Kure Atoll, Northwestern Hawaiian Islands: Atoll Research Bulletin, v. 164.

Work, T.M., Klavitter, J.L., Reynolds, M.H., and Blehert, D., 2010, Avian botulism: A case study in translocated endangered Laysan ducks (Anas laysanensis) on Midway Atoll: Journal of Wildlife Diseases, v. 46, no. 2, p. 499-506.

Work, T.M., Meteyer, C.U., and Cole, R.A., 2004, Mortality in Laysan ducks (Anas laysanensis) by emaciation complicated by Echinuria uncinata on Laysan Island, Hawaii, 1993: Journal of Wildlife Diseases, v. 40, p. 110-114.

Xie, Y., Sha, Z., and Yu, M., 2008, Remote sensing imagery in vegetation mapping: a review: Journal of Plant Ecology, v. 1 , p. 9-23.

Zhang, K., Dittmar, J., Ross, M., and Bergh, C., 2011, Assessment of sea level rise impacts on human population and real property in the Florida Keys: Climatic Change, v. 107, p. 129-146. 


\section{Appendix 1-USGS lidar (light detection and ranging) data collection specifications for the Northwestern Hawaiian Islands including Kure Atoll, Midway Atoll, Pearl and Hermes Atoll, Lisianski Island, Laysan Island, and French Frigate Shoals}

[Data were acquired July 22-25, 2010; abbreviations: <, less than; >, greater than; $\leq$, less than or equal to; $\geq$, greater than or equal to; m, meters; $\%$, percent]

\begin{tabular}{|c|c|c|}
\hline Data acquisition & Survey design & Minimum requirements \\
\hline Laser pulse rate & $\begin{array}{l}\text { Up to } 20,000 \text { pulses per second }(10,000 \text { pulses for } \\
\text { each of two instruments) }\end{array}$ & \\
\hline Returns per pulse & First and last (up to 2) & First and last (up to 2) \\
\hline On-ground laser beam diameter & Approx. 60 centimeters $(\mathrm{cm})$ & \\
\hline Scan angle & \pm 20 degrees & $\begin{array}{l}\leq \pm 20 \text { degrees (data beyond this scan angle along } \\
\text { the swath edge should not be included in the } \\
\text { final product) }\end{array}$ \\
\hline Aircraft altitude & 244-305 meters $(\mathrm{m})$ above ground & \\
\hline Aircraft speed & $85-95$ knots & \\
\hline Ground swath width & $400 \mathrm{~m}$ & \\
\hline Swath overlap & $50 \%$ sidelap ( $100 \%$ overlap) & $\begin{array}{l}\text { No voids between swaths. } \\
\text { No voids because of cloud cover or instrument } \\
\text { failure. } \\
<20 \% \text { no-overlap area per island. }\end{array}$ \\
\hline Aggregate pulse density & $\begin{array}{l}\text { 1-2/square meters }(\mathrm{m} 2) \text {; for bare areas or sparsely } \\
\text { vegetated areas, } 3-4 / \mathrm{m} 2 \text { for more densely } \\
\text { vegetated areas to increase the odds of having } \\
\text { a pulse reflect off the ground }\end{array}$ & $\begin{array}{l}1 / \mathrm{m}^{2} \text { barring nonreflective areas (for example, open } \\
\text { water): } \\
\text { - } \geq 85 \% \text { design pulse density for entire project area } \\
\text { - Within areas of swath overlap, no } 30 \times 30 \mathrm{~m} \text { area } \\
\text { with }<50 \% \text { design pulse density }\end{array}$ \\
\hline Flight line direction & $\begin{array}{l}\text { Bare areas or sparsely vegetated areas: opposing } \\
\text { parallel lines, with at least one perpendicular } \\
\text { flight line to tie parallel lines together; or } \\
\text { Densely vegetated areas: cross-hatch pattern to } \\
\text { increase pulse density }\end{array}$ & \\
\hline GPS base-line length & $\begin{array}{l}\text { Approximately } 1350 \text { kilometers }(\mathrm{km}) \text { (Midway } \\
\text { Atoll to Tern Island) }\end{array}$ & \\
\hline $\begin{array}{l}\text { GPS positional dilution of } \\
\text { precision (PDOP) }\end{array}$ & $\leq 3.0, \geq 6$ satellites in view & $\leq 3.5, \geq 6$ satellites in view \\
\hline Survey conditions & & $\begin{array}{l}\text { Cloud and fog-free between aircraft and ground. } \\
\text { No unusual flooding or inundation. }\end{array}$ \\
\hline Accuracy & Survey design & Minimum requirements \\
\hline Absolute accuracy & $<15 \mathrm{~cm}$ vertical, $<10 \mathrm{~cm}$ horizontal (RMSE) & $\leq 15 \mathrm{~cm}$ vertical (RMSE) \\
\hline Between-swath reproducibility $^{1}$ & & $\leq 15 \mathrm{~cm}$ vertical on horizontal surfaces (RMSE) \\
\hline $\begin{array}{l}\text { Reproducibility of range mea- } \\
\text { surements }\end{array}$ & & $\leq 5 \mathrm{~cm}$ (RMSE) \\
\hline
\end{tabular}

Spatial reference framework

\begin{tabular}{ll}
\hline Vertical Datum & $\begin{array}{c}\text { WGS } 84 \text { for ellipsoid heights. Local MSL for orthometric heights (will be a challenge for islands without } \\
\text { tidal gauges; heights may need to be tied to NGS benchmarks until a more accurate datum is developed } \\
\text { and orthometric heights can be adjusted). }\end{array}$ \\
\hline Horizontal Datum & NAD 83 \\
\hline Projection & UTM, zone varies among islands (for example, Midway = 1N, Laysan = 2N) \\
\hline Units & Meters (UTM) \\
\hline
\end{tabular}

${ }^{1}$ Extensive swath overlap allows for robust estimation of intra-survey reproducibility. Intra-survey measurement errors on flat ground are commonly 4-6 cm $\mathrm{RMSE}_{\mathrm{z}}$, with an increase in $\mathrm{Z}$ errors as local slope increases. ${ }^{2}$ Evaluated by measuring departures from planarity of returns from planar surfaces (for example, the lake on Laysan Island). 


\section{Appendix 2-General circulation model data downscaled for analysis of predicted temperature and precipitation}

The six general circulation models (GCM; table 1.4) analyzed for predictions of temperature and precipitation were downscaled for the Northwestern Hawaiian Islands. The models were downscaled using the delta change method (Tabor and Williams, 2010) for a scale appropriate for the NWHI. The A1B emission scenario was chosen to simulate future atmospheric concentrations of greenhouse gasses. This scenario is described as an intermediate case of emissions, where $\mathrm{CO}_{2}$ concentrations stabilize at 750 parts per million. The A1B scenario describes a future world of very rapid economic growth, low population growth, and the rapid introduction of new and more efficient technologies. Major underlying themes are convergence among regions, capacity building, and increased cultural and social interactions, with a substantial reduction in regional differences in per capita income. For each model, cell size resolution and model attributes are provided, as well as references.

\section{Appendix 3-Nesting descriptions of land birds and seabirds of the low-lying islands of the Northwestern Hawaiian Islands-Linking nesting habitat to land cover classes by island}

Species-specific nesting descriptions and land cover utilization vary by island. At Pearl and Hermes Atoll, we could not delineate habitat for Great Frigatebird and Red-footed Booby, the two shrub-nesting species known to nest here in recent years (USFWS data; Sprague, 2003). At this atoll, these two avian species have been documented nesting in relatively low-lying herbaceous vegetation such as Nelson's horsenettle (Solanum nelsonii; Amerson and others, 1974). Satelliteimagery resolution was not sufficient to classify specific plant species preventing habitat differentiation.

Land cover classification using aerial imagery did not allow for separation among soil types or bare ground substrates (for example, sand, rubble). The three burrowing species (Bonin Petrel, Wedge-tailed Shearwater, and Tristram's Storm-petrel) establish nests where they are able to excavate and the soil is stable enough to maintain a burrow; Bulwer's Petrel typically nest in crevices in coral rubble and rocks. Potential nesting habitat area for these four species is, therefore, overestimated using land cover classification because sub-surface conditions constrain where these species are able to establish a nest.

Similarly, satellite imagery resolution prevented classification of low ground cover from vine vegetation. Two endangered land birds will utilize dense vine vegetation for nesting cover, but not low ground cover where nests are exposed (Morin, 1991; Reynolds and others, 2007). Potential nesting habitat at Laysan Island is, therefore, overestimated using our land cover classification methods and ground-based methods are needed. At Pearl and Hermes Atoll, Laysan Finches utilize marine debris for nesting and occur on the beach edge; however, we could not quantify this "habitat" using satellite imagery.

Island-specific classification of human structures used for nesting habitat was necessary at Sand and Eastern Islands (Midway Atoll), and Tern Island (French Frigate Shoals). We classified buildings as habitat for White Tern at all islands as they commonly nest on window ledges and other flat surfaces off the ground (Rauzon and Kenyon, 1984). Buildings constructed on post-and-pier foundations at Tern Island were classified as nesting habitat for species that nest underneath them (Wedge-tailed Shearwater, Red-tailed Tropicbird, and Black Noddy; P. Hartzell, USFWS, written commun.). The decommissioned runway at Eastern Island is unsuitable for burrow-nesting birds because of hard-packed in-fill materials (Moore, 2009); therefore, we excluded this area from nesting habitat calculations for burrow-nesting birds (Bonin Petrel, Wedge-tailed Shearwater, and Tristram's Storm-petrel). The decommissioned runway area at Kure Atoll was also excluded as potential nesting habitat for burrow-nesters. Paved surfaces were not included as nesting habitat for any species.

Species-specific evaluations of potential nesting habitat loss accounted for inter-specific differences in vegetation classes used. However, species were generally considered as part of one of the following nesting groups, based on the type of land cover used in nesting: (1) bare ground or sparse low vegetation (Bulwer's Petrel, Gray-backed Tern), (2) tree/shrub (White-tailed Tropicbird, Red-footed Booby, Great Frigatebird, Black Noddy, and White Tern), (3) within vegetation (Bonin Petrel, Christmas Shearwater, Red-tailed Tropicbird, Laysan Teal, Laysan Finch, Nihoa Finch, Nihoa Millerbird), or (4) with vegetation and bare ground (Black-footed Albatross, Laysan Albatross, Short-tailed Albatross, Wedge-tailed Shearwater, Tristram's Storm-petrel, Masked Booby, Brown Booby, Little Tern, Sooty Tern, Brown Noddy). Habitat was not quantified for the Blue Noddy or Nihoa Finch that were found only on the higher islands of Nihoa, Mokumanamana, Gardner Pinnacles, and La Perouse Pinnacle. Breeding habitat for populations of non-native birds at Midway Atoll (Cattle Egret [Bubulcus ibis], Common Myna [Acridotheres tristis] and Common Canary [Serinus carius]) was not considered.

\section{Nesting descriptions from:}

Amerson, A.B., 1971, The natural history of French Frigate Shoals, Northwestern Hawaiian Islands: Atoll Research Bulletin, v. 150 . 
Chardine, J.W., and Morris, R.D., 1996, Brown noddy (Anous stolidus), in Poole, A., ed., The Birds of North America Online: Ithaca, New York, Cornell Lab of Ornithology.

Ely, C.A., and Clapp, R.B., 1973, The natural history of Laysan Island, Northwestern Hawaiian Islands: Atoll Research Bulletin, v. 171, p. 1-361.

Feare, C.J., 1976, The breeding of the sooty tern (Sterna fuscata) in the Seychelles and the effects of experimental removal of its eggs: Journal of Zoology, v. 179, p. $317-360$.

Fefer, S.I., Harrison, C.S., and Naughton, M.B., 1984, Synopsis of results of recent seabird research in the Northwestern Hawaiian Islands, in Grigg, R.W., and Tanoue, K.Y., Proceedings of the second symposium on resource investigations in the Northwestern Hawaiian Islands: Honolulu, Hawaii, University of Hawai'i Sea Grant College Program, p. 9-76.

Friedlander, A.K., Keller, K., Wedding, L., Clarke, A., and Monaco, M., eds., 2009, A marine biogeographic assessment of the Northwestern Hawaiian Islands: NOAA Technical Memorandum NOS NCCOS 84. Prepared by NCCOS's Biogeography Branch in cooperation with the Office of National Marine Sanctuaries Papahānaumokuākea Marine National Monument. Silver Spring, Md, 363 p.

Gauger, V.H., 1999, Black noddy (Anous minutus), in Poole, A., ed., The Birds of North America Online: Ithaca, New York, Cornell Lab of Ornithology.

Grace, J., and Anderson, D.J., 2009, Masked booby (Sula dactylatra), in Poole, A., ed., The Birds of North America Online: Ithaca, New York, Cornell Lab of Ornithology.

Lee, D.S., and Walsh-Mcgehee, M., 1998, White-tailed Tropicbird (Phaethon lepturus), in Poole, A., ed., The Birds of North America Online Ithaca, Cornell Lab of Ornithology.

Marks, J.S., and Leasure, S.M., 1992, Breeding biology of Tristram's storm-petrel on Laysan Island: The Wilson Bulletin, v. 104, no. 4, p. 719-731.

McClelland, G.T.W., Jones, I.L., Lavers, J.L., and Sato, F., 2008, Breeding biology of Tristram's storm-petrel Oceanodroma tristrami at French Frigate Shoals and Laysan Island, Northwestern Hawaiian Islands: Marine Ornithology, v. 36, p. 175-181.
Megyesi, J.L., and Griffin, C.R., 1996, Breeding biology of the Brown noddy on Tern Island, Hawaii: The Wilson Bulletin, v. 108, no. 2, p. 317-334.

Metz, V.G., and Schreiber, E.A., 2002, Great frigatebird (Fregata minor), in Poole, A., ed., The Birds of North America Online: Ithaca, New York, Cornell Lab of Ornithology.

Morin, M., 1991, The breeding ecology of the Laysan finch (Drepanidinae telespiza cantans) on Laysan Island: Honolulu, University of Hawaii Manoa, Ph.D. dissertation.

Morin, M.P., and Conant, S., 2002, Laysan finch (Telespiza cantans) and Nihoa finch (Telespiza ultima), in Poole, A., and Gill, F., eds., The Birds of North America Online: Ithaca, New York, Cornell Lab of Ornithology.

Morin, M.P., Conant, S., and Conant, P., 1997, Laysan and Nihoa millerbird (Acrocephalus familiaris), in Poole, A., ed., The Birds of North America Online: Ithaca, Cornell Lab of Ornithology.

Moulton, D.W., and Marshall, A.P., 1996, Laysan duck (Anas laysanensis), in Poole, A., ed., The Birds of North America Online: Ithaca, New York, Cornell Lab of Ornithology.

Nelson, J.B., 1978, The Sulidae: Oxford, United Kingdom, Oxford University Press.

Reynolds, M.H., 2002, The foraging ecology, population dynamics, and habitat use of Laysan teal (Anas laysanensis): Blacksburg, Va., Virginia Polytechnic and State University, Ph.D. dissertation.

Reynolds, M.H., Crampton, L.H., and Vekasy, M.S., 2007, Laysan teal Anas laysanensis nesting phenology and site characteristics on Laysan Island: Wildfowl, v. 57, p. 54-67.

Rice, D.W., and Kenyon, K.W., 1962, Breeding distribution, history, and populations of North Pacific albatrosses: Auk, v. 79 , no. 3 , p. $365-386$.

Robbins, C.S., 1966, Birds and aircraft on Midway Islands 1959-1963 investigations: Special Scientific Report: Wildlife No. 85, U.S. Fish and Wildlife Service, Washington, D.C.

Schreiber, B.A., and Schreiber, R.W., 2009, Red-tailed tropicbird (Phaethon rubricauda) in Poole, A., ed., The Birds of North America Online: Ithaca, New York, Cornell Lab of Ornithology.

Schreiber, E.A., Feare, C.J., Harrington, B.A., Murray, B.G., Robertson, W.B., Robertson, M.J., and Woolfenden, G.E., 2002, Sooty tern (Onychoprion fuscatus), in Poole, A., ed., The Birds of North America Online: Ithaca, New York, Cornell Lab of Ornithology. 
Schreiber, E.A., and Norton, R.L., 2002, Brown booby (Sula leucogaster), in Poole, A., ed., The Birds of North America Online: Ithaca, New York, Cornell Lab of Ornithology.

Schreiber, E.A., Schreiber, R.W., and Schenk, G.A., 1996, Red-footed booby (Sula sula), in Poole, A., ed., The Birds of North America Online: Ithaca, New York, Cornell Lab of Ornithology.

Slotterback, J.W., 2002, Tristram's storm-petrel (Oceanodroma tristrami), in Poole, A., ed., The Birds of North America Online: Ithaca, New York, Cornell Lab of Ornithology.

Woodward, P.W., 1972, The natural history of Kure Atoll, Northwestern Hawaiian Islands: Atoll Research Bulletin, v. 164. 
Menlo Park Publishing Service Center, California

Manuscript approved for publication, August 24, 2012 

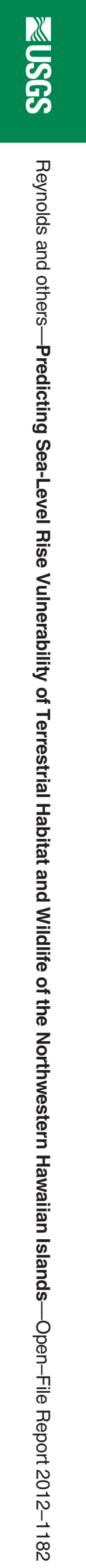